\title{
ON THE ROLE OF TOPOGRAPHY AND OF BOUNDARY FORCING IN THE OCEAN CIRCULATION
}

\author{
by \\ Paola Cessi \\ Laurea in Physics \\ University of Bologna, Italy \\ 1982 \\ Submitted in partial fulfillment of the \\ requirements for the degree of \\ Doctor of Philosophy \\ at the \\ Massachusetts Institute of Technology \\ and the \\ Woods Hole Oceanographic Institution \\ August, 1987 \\ (C) Paola Cessi 1987
}

The author hereby grants to M.I.T. permission to reproduce and to distribute copies of this thesis in whole or in part.

Signature of the author

Joint Program in Oceanography, Massachusetts Institute of Technology -

Woods Hole Oceanographic Institution, August 1987.

Certified by

Thefs supervisor

Accepted by

Chaifonan, Joint Committee for Physical Oceanography.

Massachusetts Institute of Technology -

Woods Hole Oceanographic Institution. 


\title{
ON THE ROLE OF TOPOGRAPHY AND OF BOUNDARY FORCING IN THE OCEAN CIRCULATION
}

by

Paola Cessi

Submitted to the Massachusetts Institute of Technology - Woods Hole Oceanographic Institution, Joint Program, in August, 1987, in partial fulfillment of the requirements for the degree of Doctor of Philosophy.

\begin{abstract}
This thesis consists of two loosely related problems, both of which analyze some consequences of the failure of Sverdrup relation. In the first part, Chapters 2 and 3, the Sverdrup relation is invalidated because substantial flow is obtained at the bottom where topography exists. The eddies play an essential role in transfering momentum vertically from the surface, where the forcing is applied, to the bottom, which is otherwise unforced. If the topography has a structure in the longitudinal direction, then the inviscid theory predicts the occurence of strong jets in the interior of the model ocean. According to the structure of the topography these internal jets can occur in both vertically homeogenous and baroclinic oceans. If the topographic slope changes sign, then one kind of jets is observed both in stratified and in homogeneous oceans. This phenomenon is robust to moderate amounts of dissipation and is not disturbed by the occurrence of recirculating gyres within the basin.

If the topographic slope is constant, then another kind of internal jets is observed, and it occurs in stratified models only. I was unable to observe this kind of jets in the presence of weak dissipation. The reason for this failure is twofold: on one hand friction, especially interfacial friction, tends to make the flow more barotropic (and we believe that indeed this is one of the processes that the eddies accomplish in a stratified fluid) and therefore the phenomena that rely strongly on baroclinicity are discouraged. On the other hand, reduction of the dissipation leads to the onset of a strong recirculating, inertial gyre which, although confined in space, affects the global properties of the flow.

In the second part of the thesis (Chapters 4 and 5) I developed a simple model of the recirculating, inertial gyre. Again the dynamics of this feature are far from being in Sverdrup balance. In this case inertia is responsible for the failure of Sverdrup relation, together with the eddy field which provides a mean for transfering momentum vertically and laterally into regions away from where the forcing is applied. In this model there is no direct forcing in the recirculation region, and the input of momentum is confined to the boundary currents surrounding the gyre, for example the separated Gulf Stream.

One of the results of the recirculation model is the prediction of its transport. It is shown that most of the transport is depth independent, i.e. it can be calculated
\end{abstract}


without detailed knowledge of the density structure of the ocean. It is also shown that the "barotropic" part of the transport increases as the cube of the meridional extent of the gyre.

Thesis supervisor: Joseph Pedlosky

Professor of Physical Oceanography 


\section{Acknowledgments}

My warmest thanks go to my advisor, Joseph Pedlosky, who has always been very supportive and patient with me. He has provided a remarkable example of insightful thinking and intellectual openness. I appreciated the advice from the several people who have been associated with my thesis committe: Glenn Flierl, Glenn Ierley, Paola Rizzoli, Carl Wunsch and William Young. I particularly enjoyed the very helpful discussions with Glenn Flierl. I am indebted to Glenn Ierley for generously giving me his numerical model and patiently teaching me how to use it. A special thank goes to William Young who suggested and collaborated in the problem developed in Chapters 4 and 5 , and was always very generous with his time and advice throughout my thesis preparation.

Steve Meacham has been a cheerful friend over the past few years and has helped me a lot with my FORTRAN, my asymptotic expansions and my times of discouragment.

Diana Spiegel patiently assisted me through my struggle with computers. Charmaine King produced the nicest computer plots in the thesis. Anne-Marie Michael relieved me of some of the thesis typing.

The thesis work has been supported by a National Foundation grant from the Office of Atmospheric Sciences. Most of the numerical calculations were performed on computers located at the National Center for Atmospheric Research. NCAR is funded by the National Science Foundation. 


\section{Table of contents}

Abstract. . . . . . . . . . . . . . . . . . . . . . 2

Acknowledgments. . . . . . . . . . . . . . . . . . . . . . . . . 4

Table of contents. . . . . . . . . . . . . . . . . . . . . 5

Chapter 1: Introduction

1.1 Homogeneous models with topography. . . . . . . . . . . . . . . . 8

1.2 Wind driven circulation in baroclinic oceans. . . . . . . . . . . . 10

1.3 The effect of the transient eddies on the mean circulation. . . . . . . 17

1.4 Early theories of the inertial circulation. . . . . . . . . . . . . 20

1.5 Overview of the thesis. . . . . . . . . . . . . . . . 22

References for Chapter 1. . . . . . . . . . . . . . . 25

Chapter 2: On the role of topography in the wind-driven circulation: the inviscid limit

2.1 Introduction. . . . . . . . . . . . . . . . . . 27

2.2 Two-layer model. . . . . . . . . . . . . . . . . . . . 29

2.3 Three-layer model. . . . . . . . . . . . . . . . . . . . . 44

2.4 Continuous stratification model. . . . . . . . . . . . . . . 49

2.5 Ridge-like topography. . . . . . . . . . . . . . . 58

2.6 Discussion. . . . . . . . . . . . . . . . . . . . . . . . . 64

A2. Viscous boundary layer analysis. . . . . . . . . . . . . 66

B2. Homogeneous model in the presence of topography. . . . . . . . 69

C2. The constant value of potential vorticity within the "bowl". . . . 71

Chapter 3: On the role of topography in the wind-driven circulation: viscous and inertial effects

3.1 Numerical experiments. . . . . . . . . . . . . . . . . . 74

3.2 Ridge-like topography. . . . . . . . . . . . . . . . 78

3.3 Constant slope topography. . . . . . . . . . . . . . . . . . . 87

3.4 Summary and discussion. . . . . . . . . . . . . . . . . . . . . 104

A3 Description of the numerical model. . . . . . . . . . . . 107

References for Chapters 2 and 3 . . . . . . . . . . . . . . . . 109

Chapter 4: A model of the inertial recirculation driven by boundary currents: the homogeneous model

4.1 Introduction.

4.2 Formulation of the barotropic model. . . . . . . . . . . . . . . 123

4.3 Homogenized gyre filling the box. . . . . . . . . . . . . . . 126

4.4 Homogenized gyres with free boundaries. . . . . . . . . . . . . 134

4.5 A comment on the zonal scale of penetration. . . . . . . . . . . . 139

4.6 Summary and discussion. . . . . . . . . . . . . . . . . . . . . 142

A4. Derivation of the velocity "weighted average" (4.3.2). . . . . . . 145

B4. How to find $\bar{q}$ without solving for the flow. . . . . . . . . . . 146

C4. Homogenized gyre filling an elliptical domain. . . . . . . . 147 
Chapter 5: A model of the inertial recirculation driven by boundary currents: the stratified model

5.1 Introduction.

5.2 Formulation of the baroclinic model and general results. . . . . . 152

5.3 One and a half layer model. . . . . . . . . . . . . . . . . . 165

5.4 Analytical solutions of the two layer model. . . . . . . . . . . . 169

5.5 Numerical results. . . . . . . . . . . . . . . . . . . 178

5.6 Summary and conclusions. . . . . . . . . . . . . . . . 186

A5. Approximate solutions for the gyres widths $\ell_{1}$ and $\ell_{2}$. . . . . . 188

References for Chapters 4 and 5. . . . . . . . . . . . . . . . 191

Summary and conclusions. . . . . . . . . . . . . . . . . . 192 


\section{CHAPTER 1}

\section{Introduction}




\subsection{Homogeneous models with topography.}

One of the most widely investigated problems in ocean dynamics is the wind driven circulation. With important exceptions, which I will describe later, all models of the steady wind driven circulation rely on the validity of the Sverdrup relation in the interior of the ocean. This relation gives the vertically integrated meridional transport once the stress, $\vec{\tau}=\left(\tau_{x}, \tau_{y}\right)$, by the wind is known, i.e.

$$
\beta \int_{-H}^{0} d z \rho v=\left(\frac{\partial \tau_{y}}{\partial x}-\frac{\partial \tau_{x}}{\partial y}\right)
$$

Here $v$ is the meridional velocity, $\beta$ is the planetary vorticity gradient and $\rho$ is the density. The relation (1.1.1) is remarkable because it's independent of the details of the vertical stratification, but fails if there is a vertical velocity at the bottom $z=-H$. Such bottom velocities need not to be very large to disrupt the Sverdrup relation. Indeed they need only to be of the same order as the surface Ekman velocity (proportional to the right hand side of $(1.1 .1)$ ) which doesn't exceed $10^{-4} \mathrm{~cm} / \mathrm{sec}$. Vertical velocities at the bottom of the ocean can be generated if the abyssal currents impinge on topographic reliefs. Although bottom slopes are pervasive in all oceanic basins, abyssal currents are generally considered to be so small in the interior that they produce negligible vertical velocities. Since typical bottom slopes are of the order of $1 \mathrm{~m} / \mathrm{km}$, horizontal bottom velocities need only to be $1 \mathrm{~mm} / \mathrm{sec}$ to produce vertical velocities as large as the highest, wind generated, Ekman pumping. Therefore bottom topography can play an important role in the determination of the vertically averaged meridional transport. Indeed this seems to be the case in the portion of the North Atlantic subpolar gyre analyzed by Luyten, Stommel and Wunsch (1985).

One of the early attempts of estimating the effects of bottom topography in the wind driven circulation is the vertically homogeneous model of Holland (1967). In his model the ocean is enclosed in a basin of simple geometry and the flow is driven by 
a simple Ekman pumping at the surface. Away from the solid boundaries, the flow is governed by

$$
J\left(\Psi, \frac{f}{h}\right)=\frac{\partial}{\partial x}\left(\frac{\tau_{y}}{h}\right)-\frac{\partial}{\partial y}\left(\frac{\tau_{x}}{h}\right)
$$

where $\Psi$ is the mass transport streamfunction, and is related to the velocity field by $\Psi_{x}=\rho v h, \Psi_{y}=-\rho u h . f=f_{0}+\beta y$ is the Coriolis parameter and $h$ is the variable depth of the model ocean, of constant density $\rho$. Frictional and inertial effects are confined mostly to regions close to the boundaries. In the presence of topography of varying slope, internal boundary layers can arise, where friction and inertia become important. Holland (1967) does not discuss the occurrence of internal boundary layers. A detailed discussion will be found in section 2.5 of the present work.

An important consequence of the inclusion of topography can be readily seen by inspection of (1.1.2): the free geostrophic contours, along which the flow would be constant in the absence of forcing, are not latitude circles (parallel to $f$ ) as in (1.1.1). Even when the topographic relief is small compared to the total ocean depth the effect is very noticeable. If $h=H-h_{b}$, where $H$ is the average ocean depth and $h_{b}$ is the bottom elevation, when $h_{b} \ll H$ we have $f / h \approx f_{0} / H+\beta y / H+f_{0} h_{b} / H^{2}$ and (1.1.2) becomes

$$
J\left(\Psi, \beta y+f_{0} h_{b} / H\right)=\frac{\partial \tau_{y}}{\partial x}-\frac{\partial \tau_{x}}{\partial y} \equiv \operatorname{curl} \tau
$$

An example of circulation in the presence of simple topography is shown in Fig. 1.1a and it should be compared with the circulation obtained with the same forcing in the absence of bottom elevation, shown in Fig. 1.1b. With this simple constant slope topography, the maximum transport value is reduced and is shifted northward. In the above example a very idealized form of topography has been shown, Holland (1967) analyzed various types of more complicated topography. In Fig. 1.2 contours of $h / \sin$ (latitude) (parallel to $f / h$ ) for the North Atlantic are shown. Indeed the topographic control of the geostrophic contours is very strong and one would expect strong deviation from the 
Sverdrup prediction (1.1.1). But the ocean is far from being vertically homogeneous and bottom flow may be much slower than the surface flow so that the topographic effect may be reduced. A major part of this thesis concerns the interaction of stratification with topography.

\subsection{Wind driven circulation in baroclinic oceans}

Welander (1968) analyzed a two layer model in the presence of topography and wind forcing. He included dissipation in the form of stress at the interface between the two layers (proportional to the interface velocities) and of bottom friction (proportional to the bottom velocities). In his model the lower layer is supposed to be so much deeper than the upper layer that no appreciable interface deformation occurs in the interior. The lower layer is forced by the interfacial stresses which are of order one only near the western wall, where the upper layer western boundary layer induces strong velocities that drive the abyssal flow. Topography plays an essential role only if it is strong enough to reverse the gradients of the geostrophic contours, that is, if it creates regions where $\left(f / h_{2}\right)_{y}<0\left(h_{2}\right.$ is the variable depth of the lower layer). If this is the case the abyssal circulation generated near the western wall cannot be closed within the western boundary since a linear frictional boundary layer is not allowed there. Instead the circulation must flow through the interior to reach the eastern wall and thus close the circulation.

In this case the flow is divided into three regions:

1) A region near the western wall where the forcing $\mathcal{F}$, provided by interfacial friction, is of order 1 and the flow is governed by ( $\psi$ is the lower layer transport streamfunction)

$$
J\left(\psi, f / h_{2}\right)=\mathcal{F}
$$



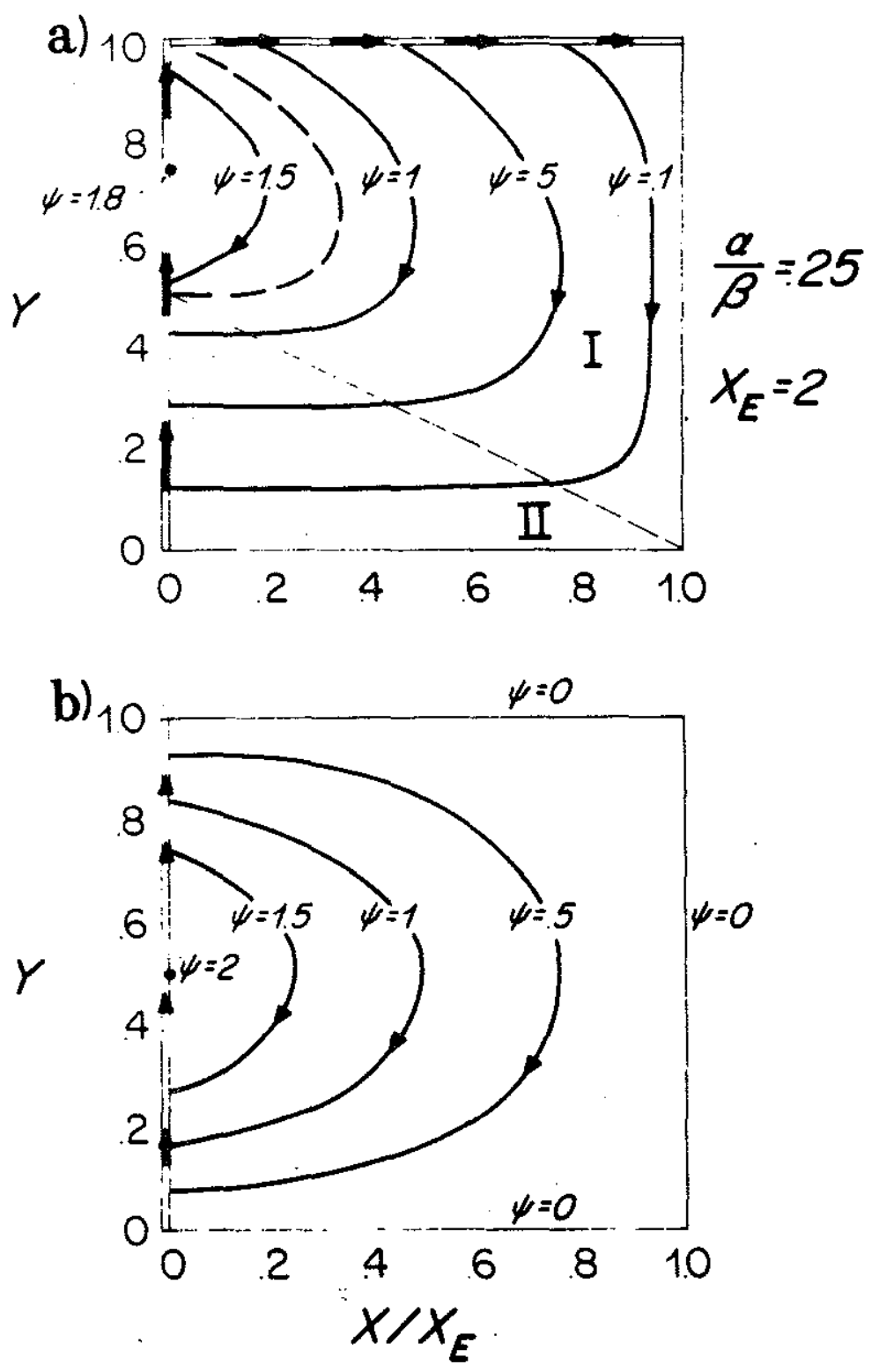

Fig. 1.1: Solution of (1.1.3) for curl $\tau=\sin \pi y$ and $f_{0} h_{b} / H=\alpha x$ (reproduced from Pedlosky,1986). a) $\alpha=\beta / 4$, a schematic boundary layer circulation is shown on the northern as well as on the western boundary. b) $\alpha=0$, the circulation is symmetric in the meridional direction. 


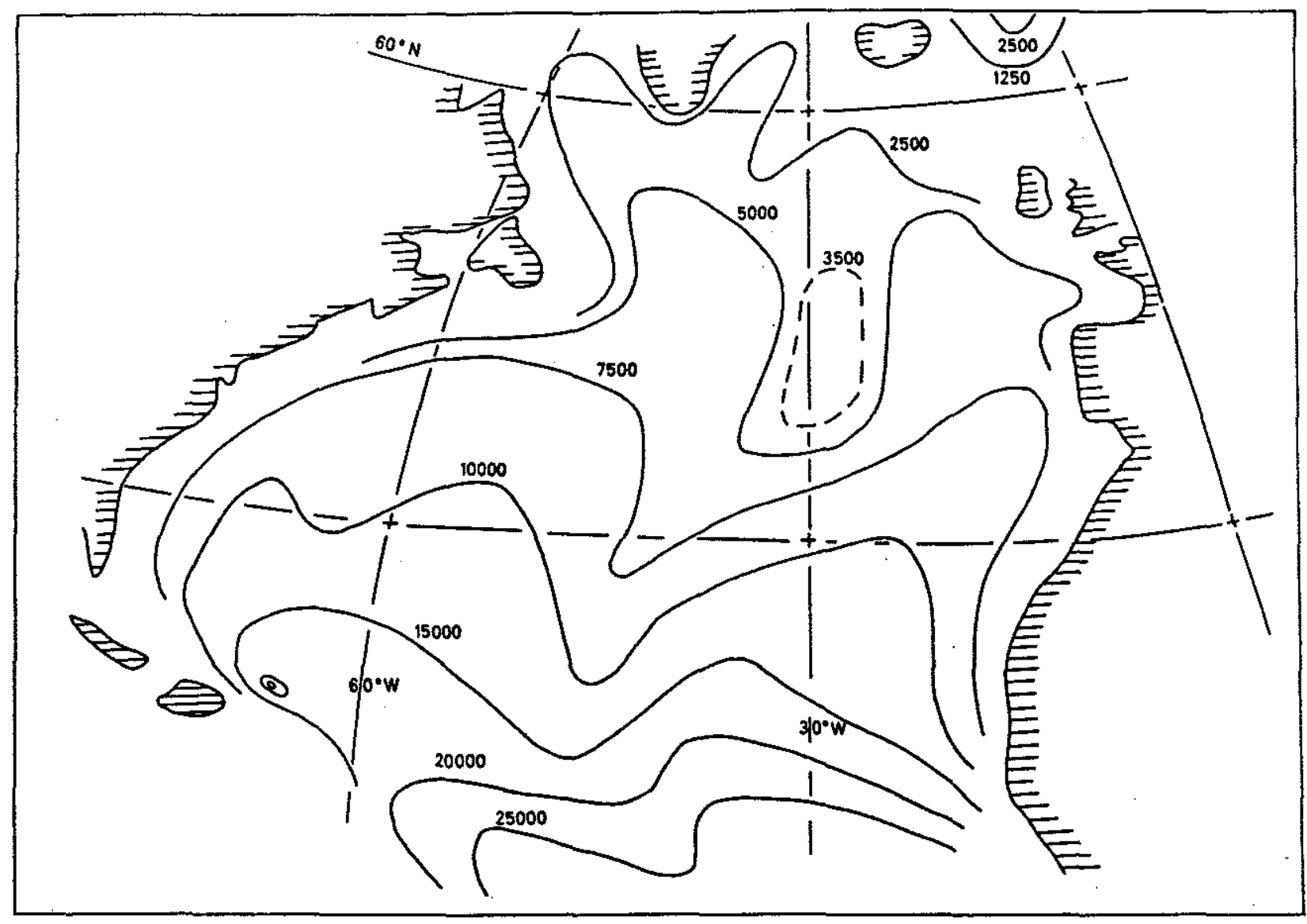

Fig. 1.2 Contours of $h / \sin ($ latitude) for the North Atlantic. (Reproduced from Welander, 1968) 
2) An interior region where the forcing is small, say of order $\kappa$, and dissipation is small, say of order $\delta$, and the flow occurs along the geostrophic contours

$$
J\left(\psi, f / h_{2}\right)=0
$$

The free flow in this interior region is determined by matching with the "upstream" flow of region 1) (in this case from west to east), and is going to be of the same strength as in region 1).

3) An eastern boundary layer region where dissipation is essential and the circulation is frictionally closed.

$$
J\left(\psi, f / h_{2}\right)=-\delta \nabla^{2} \psi
$$

Another interesting case occurs in Welander's (1968) model when topography distorts the geostrophic contours so that they close upon themselves in the interior (for example the dashed contour in Fig. 1.2). When this is the case the flow within the geostrophic contours can be as large as in the wind forced upper layer, even though the direct forcing due to the interfacial stresses is weak. The reason for this anomalous behaviour will be illustrated here. The region of closed contours belongs to the interior where forcing and dissipation can be neglected to a first approximation. Therefore (1.2.2) is valid and the flow will be along the geostrophic contours $\psi=\mathcal{G}\left(f / h_{2}\right)$. Such a free flow cannot be determined by the "upstream" flow since the geostrophic contours close upon themselves. On the other hand, integrating the full vorticity equation over the area enclosed by any closed geostrophic contour we get

$$
\begin{aligned}
& \delta \int d A \nabla^{2} \psi=\delta \oint \vec{\nabla} \psi \cdot \hat{n} d \ell=\int d A \mathcal{F} \\
& \text { where } \quad \hat{n}=\frac{\vec{\nabla}\left(f / h_{2}\right)}{\left|\vec{\nabla}\left(f / h_{2}\right)\right|}
\end{aligned}
$$

In the interior region the forcing is small, $\mathcal{F}=\kappa \mathcal{F}_{l}$, and so is the dissipation therefore

$$
\vec{\nabla} \psi \simeq \frac{\mathrm{d} \mathcal{G}}{\mathrm{d}\left(f / h_{2}\right)} \vec{\nabla}\left(f / h_{2}\right) .
$$


Since $\mathcal{G}$ is constant along a geostrophic contour it can be taken outside the integral and (1.2.4) becomes

$$
\frac{\mathrm{d} \mathcal{G}}{\mathrm{d}\left(f / h_{2}\right)}=\frac{\kappa \int d A \mathcal{F}_{0}}{\delta \oint\left|\vec{\nabla}\left(f / h_{2}\right)\right| d \ell} .
$$

This shows that the flow inside the closed geostrophic contours is order $\kappa / \delta$, and thus not necessarily small.

As stated earlier, Welander (1968) excluded the possibility of strong distortion of the interface due to the upper layer motion. However Rhines and Young (1982a,b) have shown that this is a primary mechanism for inducing motion in the subsurface waters. They considered a problem very similar to Welander's (1968), but in the context of quasigeostrophic dynamics and in the absence of bottom elevation. For a two layer system forced by Ekman pumping $w_{e}$ and dissipated by lateral friction and bottom drag the relevant equations are

$$
\begin{aligned}
& J\left(\psi_{1}, q_{1}\right)=f_{0} w_{e} / H_{1}+\kappa \nabla^{2} q_{1} \\
& J\left(\psi_{2}, q_{2}\right)=\kappa \nabla^{2} q_{2}-\delta \nabla^{2} \psi_{2} .
\end{aligned}
$$

In the interior relative vorticity can be neglected and the vorticity is given by

$$
\begin{aligned}
& \qquad \begin{array}{l}
q_{1}= \\
q_{1}\left(\psi_{2}-\psi_{1}\right)+\beta y \\
q_{2}=F_{2}\left(\psi_{1}-\psi_{2}\right)+\beta y \\
F_{1}=f_{0}^{2} /\left(g^{\prime} H_{1}\right) \text { and } F_{2}=F_{1} H_{1} / H_{2}
\end{array}
\end{aligned}
$$

In the absence of relative vorticity and bottom elevation the Sverdrup relation (1.1.1) holds in the interior, i. e.:

$$
\beta\left(H_{1} \psi_{1}+H_{2} \psi_{2}\right)_{x}=f_{0} w_{e}
$$

(bottom friction can be neglected) and the vertically integrated transport is readily found

$$
H \psi_{b} \equiv H_{1} \psi_{1}+H_{2} \psi_{2}=\left(f_{0} / \beta\right) \int_{x_{e}}^{x} d x w_{e}
$$


where $x_{e}$ is the eastern boundary of the basin, and the problem now is to determine the vertical structure of the circulation.

In the interior the lower layer flow is given by

$$
J\left(\psi_{2}, \beta y+F_{2} \psi_{b} H / H_{1}\right)=0
$$

which has as a solution

$$
\psi_{2}=\mathcal{G}\left(\beta y+F_{2} \psi_{b} H / H_{1}\right)
$$

and should be compared to (1.2.2). As in Welander's model the geostrophic contours are known and, unless they close, the solution is determined by the "upstream" flow. If the distortion due to the wind driven motion (1.2.8) is small, the geostrophic contours will be essentially along latitude circles and the "upstream" flow is at the eastern boundary where $\psi_{1}=\psi_{2}=0$. At the eastern boundary we get $\mathcal{G}(\beta y)=0$ and the only solution to (1.2.9) is $\psi_{2}=0$ everywhere. If the Sverdrup circulation is strong enough, some geostrophic contours will not reach the eastern boundary, but they will "close" inside the western boundary layer. Then a region very similar to the region of topographically closed geostrophic contour described by Welander (1968) will arise. In this area the motion will be determined by an integral balance of the type given by eqations 1.2.4 and 1.2.5. Integrating (1.2.6b) over the area enclosed by any such closed contour we find

$$
\delta \oint \vec{\nabla} \psi_{2} \cdot \hat{n} d \ell=\kappa \oint \vec{\nabla} q_{2} \cdot \hat{n} d \ell
$$

It is convenient to write $q_{2}=\beta y+F_{2}\left(\psi_{b}-\psi_{2}\right) H / H_{1} \equiv \hat{q}_{2}-F_{2} \psi_{2} H / H_{1}$ so that (1.2.10) becomes

$$
\left(\delta+\kappa F_{2} H / H_{1}\right) \oint \vec{\nabla} \psi_{2} \cdot \hat{n} d \ell=\kappa \oint \vec{\nabla} \hat{q}_{2} \cdot \hat{n} d \ell
$$

$\hat{q}_{2}$ are the geostrophic contours and the integrals are performed along a line of constant $\hat{q}_{2}$. From (1.2.9b) $\vec{\nabla} \psi_{2}=d \mathcal{G} / d \hat{q}_{2} \vec{\nabla} \hat{q}_{2}$ and (1.2.11) becomes

$$
\begin{gathered}
\frac{\mathrm{d} \mathcal{G}}{\mathrm{d} \hat{q}_{2}}=\frac{\kappa}{\delta+\kappa F_{2} H / H_{1}} \\
\text { or } \hat{q}_{2}-F_{2} H / H_{1} \psi_{2} \equiv q_{2}=(\delta / \kappa) \psi_{2}+\text { constant }
\end{gathered}
$$


In the absence of bottom friction this gives that potential vorticity is homogenized within the closed geostrophic contours, and in this region the abyssal flow is of the same order as the surface flow. Thus the lower layer is divided in two regions: in the open contours region the flow is very weak, of order $\kappa$, in the closed contours region the flow is of order 1. In both regions the Sverdrup relation (1.1.1) or (1.2.7) holds. The purpose of the first part of this thesis (Chapters 2 and 3 ) is to analyze some of the effects of bottom topography, and the consequent failure of the Sverdrup relationship, on the wind driven circulation, in the framework of the ideas developed by Rhines and Young $(1982 \mathrm{a}, \mathrm{b})$. It will be shown that topography induces strong jets in the interior of the basin, when the inviscid limit is considerd. Two kinds of internal jets arise in the presence of longitude dependent topography. The first kind occurs only in baroclinic models, even in the presence of constant slope topography. The second kind occurs both in baroclinic and barotropic models, but requires topography of varying slope. The first kind of jets is destroyed by the presence of moderate dissipation, while the second kind survives to moderate amount of dissipation. 


\subsection{The effect of the transient eddies on the mean circulation}

In the derivation of the result (1.2.12) it is crucial that the "forcing" for the subsurface waters is provided by weak vertical diffusion of horizontal momentum $\left(\kappa \nabla^{2} F_{2}\left(\psi_{1}-\psi_{2}\right)\right.$ in the layer approximation). Such a form of diffusion, though, is not molecular diffusion, nor the effect of small structure turbulence, but represents a parameterization of the effect of transient eddies (motions on the scale of the Rossby deformation radius) on the mean, time averaged, large scale circulation. A rigorous, deductive argument, justifying the type of parameterization indicated in Rhines and Young (1982a), cannot be given. Some tentative arguments, and an extensive list of references can be found in Young (1987). The strength of the result (1.2.12) comes mostly from supporting evidences supplied by Eddy Resolving General Circulation models (Holland et al. 1984) and oceanic data analysis (McDowell et al. 1983). In particular, wind driven ERGCM's show that, once the transient component is averaged out, the time mean circulation in the unforced layer occurs in a region where potential vorticity is homogenized (see Fig. 1.3). A noticeable feature of the circulation shown in Fig. 1.3 is the intense gyres occurring in the deepest layer (Fig. 1.3e) directly south and north of the region where the boundary current separates. In such a region, (see Schmitz, 1978, and Schmitz and Holland, 1986) the eddy field is particularly vigorous and is conceivable that it acts as a source of both "forcing" and "dissipation" for the mean circulation.

The intense recirculating gyre observed in ERGCMs is associated with an analogous feature observed particularly in the North Atlantic. The circulation diagram proposed by Worthington (1962), shows, among other things, an intense gyre flowing anticyclonically south of the separated Gulf Stream and recirculating about $120 \mathrm{~Sv}$. In Worthington's diagram about half of the transport occurs below the thermocline (potential temperature smaller than $4^{\circ} \mathrm{C}$ ), and extends all the way to the bottom. More recent observations, based mostly on direct current measurements, have qualitatively confirmed Worthington's picture, showing deep velocity of about $6 \mathrm{~cm} / \mathrm{sec}$ (Schmitz, 

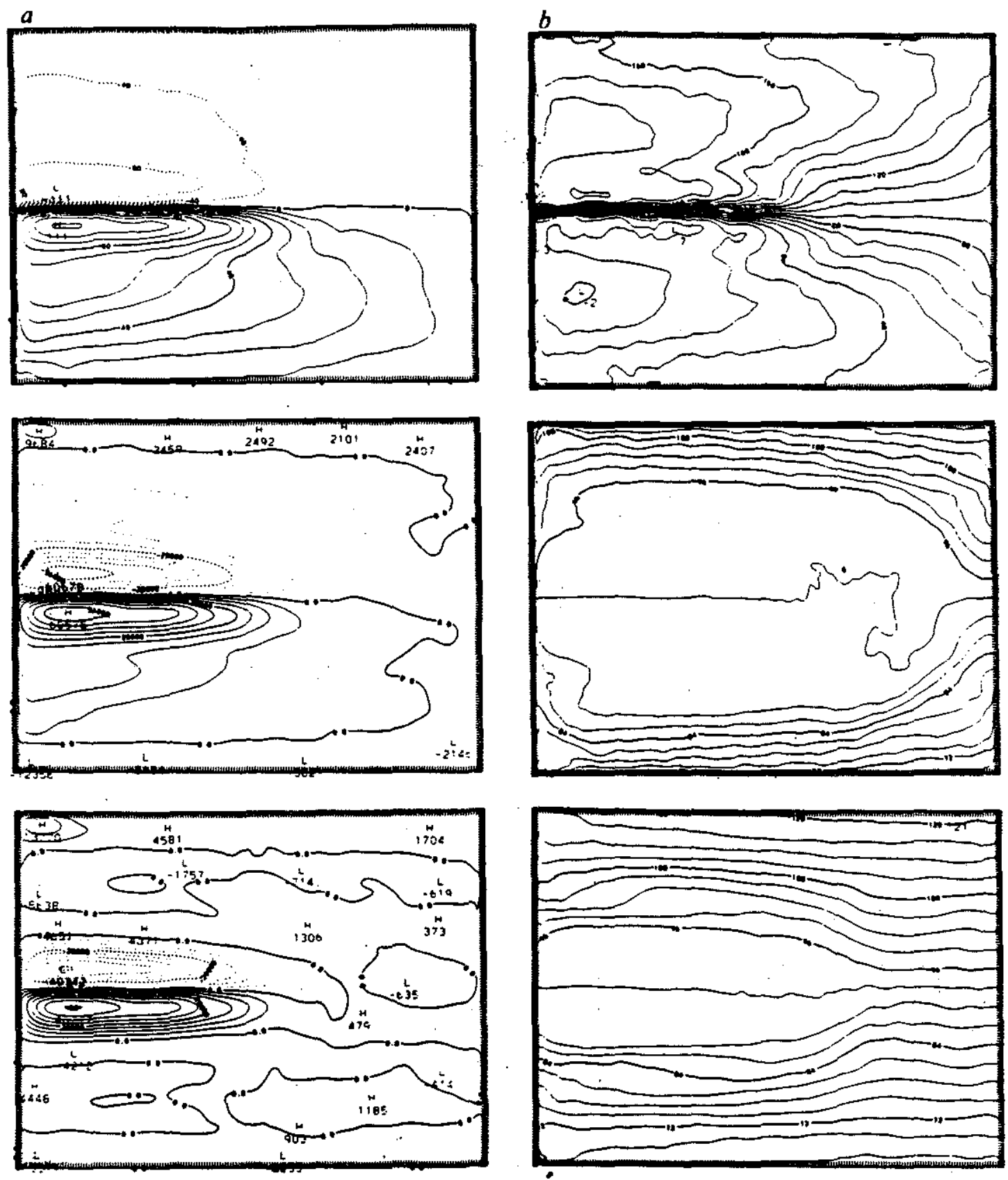

Fig. 1.3: The time averaged streamfunction and potential vorticity fields resulting from the wind driven, quasigeostrophic, eight layer ERGCM of Holland et al. (1984) at different depths. a) Streamfunction at $150 \mathrm{~m}$, b) potential vorticity at $150 \mathrm{~m}$. c) Streamfunction at $850 \mathrm{~m} \mathrm{~d}$ ) potential vorticity at $850 \mathrm{~m}$, e) Streamfunction at $1750 \mathrm{~m}$, f) potential vorticity at $1750 \mathrm{~m}$. 
1980 ,Richardson, 1985). The estimated transport for the water recirculated south of the Gulf Stream is far from being in Sverdrup balance with the observed wind system, and in the recirculation region (1.1.1) fails.

Before reviewing some of the earlier theories relevant to the circulation in this region, I will state the point of view that will be developed in Chapters 4 and 5 of this thesis: the recirculating gyre is not directly wind or thermally forced, but is driven by the eddy field present in this region. More specifically the transient eddies provide (on average) the vehicle for transmitting energy and momentum to the gyre. Similarly to Rhines and Young (1982a) the eddy field will transfer momentum vertically from the surface to the bottom, driving the circulation in regions that are not directly forced. Moreover, the eddy field will transfer momentum laterally, from the energetic boundary current system (e.g. the Gulf Stream) to the interior of the gyre which is otherwise unforced.

In order for eddies to transfer momentum laterally, inertia must become important for the mean flow. Where this is the case, the transient eddies and inertia will provide a way, alternative to bottom topography, to disrupt the Sverdrup relation. 


\subsection{Early theories of the inertial circulation}

The first work assessing the effects of inertia in the ocean circulation is Fofonoff's (1954). Actually, the focus of his model was not the recirculating gyre flanking the Gulf Stream, since observations were not available at the time. His free, inertial circulation fills the whole basin and the inertial effects are confined to boundary layers on the western, northern, and eastern walls (in the subtropical gyre). A major concern in Fofonoff's point of view is to fulfill the conditions under which inertial effects are confined to the edge of the gyre as described above. This point is particularly stressed in the subsequent work of Niiler (1966).

In Niiler's model, weak forcing (induced by the wind stress) and weak dissipation (in the form of bottom friction) are taken into account to remove the indeterminacy associated with free, inertial flows. More specifically, the free flow satisfies an equation similar to $(1.2 .1)$ or $(1.2 .9)$, i.e.

$$
q=\mathcal{Q}(\psi) \text { where } \quad q=\nabla^{2} \psi+\beta y
$$

The functional form, $\mathcal{Q}$, is determined by requiring that an integral balance similar to $(1.2 .10)$ is satisfied

$$
f_{0} / H \int d A w_{e}=\delta \oint \vec{\nabla} \psi \cdot \hat{n} d \ell
$$

where the integrals are performed on any closed streamline (all the streamlines must be closed since the flow is contained in a closed basin). A point raised by Niiler is particularly relevant to the analysis developed in Chapters 4 and 5 . He claims that parameterization of the eddy field as lateral diffusion $\left(\kappa \nabla^{2} q\right)$ is inconsistent with the existence of weakly forced and dissipated inertial flows. It seems to me that this is the case only if one insists on having inertia confined to boundary layers. For gyres that are strongly nonlinear $\left(\nabla^{2} \psi\right.$ comparable to $\left.\beta y\right)$ in the interior this is not the case. 
If lateral diffusion of potential vorticity replaces bottom friction the integral balance (1.4.2) becomes

$$
f_{0} / H \int d A w_{e}=-\kappa \oint \vec{\nabla} q \cdot \hat{n} d \ell
$$

In the subtropical gyre, for example, $w_{e}$ is always negative and the wind induced Sverdrup circulation is also negative (anticyclonic). If the forcing and the dissipation are weak then, to first order, (1.4.1) holds and $\vec{\nabla} q=(d \mathcal{Q} / d \psi) \vec{\nabla} \psi$, and (1.4.3) becomes

$$
\frac{\mathrm{d} \mathcal{Q}}{\mathrm{d} \psi}=-\frac{f_{0} / H \int d A w_{e}}{\kappa \oint|\vec{\nabla} \psi| d \ell}
$$

If one seeks a solution which consists of one anticyclonic gyre, flowing in the same sense as the weak Sverdrup flow, then the denominator in (1.4.4) is negative and thus $d \mathcal{Q} / d \psi<0$. A contrived example of such circulation is based on the circular modon analyzed by Stern (1975). Suppose we consider, for geometrical simplicity, a circular basin of radius $R$. Following Niiler (1966), one can impose a solution $q=\mathcal{Q}(\psi)$ first and then construct an appropriate wind forcing which satisfies (1.4.4). The simplest form for $\mathcal{Q}$ is $\mathcal{Q}=-\lambda^{2} \psi+\bar{q}$ and the flow satisfies

$$
\nabla^{2} \psi+\beta y=-\lambda^{2} \psi+\bar{q}
$$

with boundary condition $\psi=0$ on $r=R$.

The solution is

$$
\lambda^{2} \psi=\bar{q}\left[1-\frac{J_{0}(r \lambda)}{J_{0}(R \lambda)}\right]-\beta \sin \theta\left[r-R \frac{J_{1}(r \lambda)}{J_{1}(R \lambda)}\right]
$$

where $J_{n}$ are Bessel functions of integer order. Because the boundary of the vortex is a solid wall, the radius $R$ can be prescribed, unlike the solution analyzed by Stern (1975). If, for example, $R \lambda=2$, then (1.4.6) consists of one anticyclonic gyre if $\bar{q}<-0.24 \beta R$. The simplest way to construct an Ekman pumping which satisfies (1.4.4) is to take $f_{0} w_{e} / H=\kappa \lambda^{2} \nabla^{2} \psi$ that is

$$
f_{0} w_{e} / H=\kappa \lambda^{2}\left[\bar{q} \frac{J_{0}(r \lambda)}{J_{0}(R \lambda)}-\beta R \sin \theta \frac{J_{1}(r \lambda)}{J_{1}(R \lambda)}\right]
$$


In the solution (1.4.6), inertial effects are not confined to narrow boundary layers as in Fofonoff's gyre, but they invade the whole basin. While the predominance of inertial effects may not be a realistic feature of the whole subtropical gyre, it is very likely to be relevant for the dynamics of the recirculating gyre which occupies a subbasin scale region. In Chapters 4 and 5 it will be shown that a gyre with characteristics very similar to those observed in ERGCMs and in the North Atlantic, can be obtained with a simple, analytically tractable model. Unlike Fofonoff's model, inertial effects will be important everywhere in the gyre, both in the eastward and in the westward flow.

\subsection{Overview of the thesis}

In the first part of the thesis, Chapters 2 and $3, I$ analyze what happens when the circulation driven by the wind reaches the ocean floor, where topographic relief is present. If the abyssal waters are set in motion by weak vertical momentum transfer, from the wind forced surface waters to the bottom waters, the abyssal flow will be substantial only in regions where the geostrophic contours are closed. In the limit of infinitesimal interfacial stress, the abyssal flow outside the closed geostrophic contours is zero. In the presence of bottom relief which depends on longitude, this leads to a mismatch of the flow along some portion of the abyssal flow boundary, and this is a region which is typically in the basin interior. This problem is dealt with in Section 2.2, where it is shown that the flow mismatch arises regardless of the detailed process which forces the abyssal layer, as long as such forcing is weak. It appears, though, that the occurrence of these internal flow mismatches is discouraged by the presence of a finite amount of dissipation and interfacial friction. The role of finite diffusion is discussed in Section 3.3.

Internal mismatches of a different kind arise in the presence of topography of varying slope, such as a ridge-like relief. The production of these internal boundary layers can occur both in vertically homogeneous and in stratified models, and the flow 
over ridge-like topography is analyzed in Section 2.5. Unlike the mismatch discussed in Section 2.2, this kind of jets is robust to the inclusion of dissipation, as shown by the results presented in Section 3.2.

In the second part of the thesis, Chapters 4 and 5 , a simple model of the recirculationg gyre, responsible for the enhanced transport of the separated Gulf Stream, is presented. The model gyre is highly inertial and is forced by a potential vorticity anomaly applied at the edge of the gyre. This boundary forcing mimics the effect of the boundary currents carrying anomalous values of potential vorticity generated in latitudes away from the recirculating gyre. This choice is justified in Section 4.1. The localized forcing is tranmitted to the interior of the gyre through the weak action of the eddies which act, on average, like lateral diffusion. Chapter 4 analyzes a vertically homogeneous model and in Section 4.4 a formula is derived which relates the maximum transport of the gyre to its meridional size: the transport is proportional to the cube of the gyre width. Unlike earlier theories of the inertial circulation, in this model inertia is not confined to a thin, boundary layer region of eastward flow, but it's important everywhere, including the westward flowing region. Moreover the North-South width of the eastward flowing region is not thin, but is half the width of the westward flowing region.

The choice of a homogeneous model for the recirculation is justified by oceanic observations which show that this flow is "weakly depth dependent". Nevertheless it is desirable to obtain this characteristic as a result of a model rather than to state it as an a priori assumption. This is why the calculations of Section 4.4 are extended to include stratification in Chapter 5. Indeed the results of Section 5.4 confirm that the baroclinic structure of the recirculating gyre is confined to thin boundary layers at the edge of the gyre. The center of the recirculation is dominated by a "barotropic core" which carries most of the transport. The relation of the "barotropic core" transport to the gyre width is the same as that found in the homogeneous model of Section 4.4. A 
rationalization for the weak depth dependence of the gyre is given at the end of Section 5.2 .

The two parts of the thesis are connected by the common theme of flow within closed streamlines induced by the weak forcing provided by the eddy field. Although the forcing is weak, the response is strong in some regions, and can give rise to unexpected flow structures which break the usual Sverdrup balance. The thesis, though is written in two separated parts, and Chapters 4 and 5 can be read independently of Chapters 2 and 3. 


\section{References}

Fofonoff, N.P.,1954: Steady flow in a frictionless homogeneous ocean. J. Mar. Res., 13, 354-262.

Holland, W.R., T. Keffer and P.B. Rhines, 1984: Dynamics of the oceanic general circulation, the potential vorticity field. Nature, 308, 698-705.

Luyten, J.L., H. Stommel and C.I. Wunsch, 1985: A dignostic study of the Northern Atlantic subpolar gyre. J. Phys. Oceanogr., 15, 1344-1358.

Mc Dowell, S., P.B. Rhines and T. Keffer, 1983: Maps of North Atlantic potential vorticity and its relation to the general circulation. J. Phys. Oceanogr., 12, $1417-$ 1436.

Niiler, P.P., 1966: On the theory of wind-driven circulation. Deep Sea Res., 13, 597606.

Pedlosky, J., 1986: Geophysical Fluid Dynamics. Springer Verlag, New York, 710 pp.

Rhines, P.B. and W.R. Young, 1982a: A theory of wind-driven circulation I. Mid-ocean gyres. J. Mar. Res., 40 (Suppl.), 559-596.

Rhines, P.B. and W.R. Young, 1982b: Homogenization of potential vorticity in planetary gyres. J. Fluid Mech., 122, 347-367.

Richardson, P.L., 1985: Average velocity and transport of the Gulf Stream near $55^{\circ}$ W. J. Mar. Res., 43, 83-111.

Schmitz, W.J., 1980: Weakly depth-dependent segments of the North Atlantic circulation. J. Mar. Res., 38, 111-133.

Schmitz, W.J. and W.R. Holland, 1986: Observed and modeled mesoscale variability near the Gulf Stream and Kuroshio extention. J. Geophys. Res., 91, 9624-9634.

Stern, M.E., 1975: Minimal properties of planetary eddies. J. Mar. Res., 33, 1-13.

Welander, P., 1968: Wind-driven circulation in one- and two-layer oceans of variable depth. Tellus, 20, 1-15.

Worthington, L. V., 1962: Evidence for a two gyres circulation system in the North Atlantic. Deep Sea Res., 9, 51-57.

Young, W.R., 1987: Baroclinic theories of wind driven circulation. In General circulation of the ocean edited by H.D.I. Abarbanel and W.R. Young. Springer-Verlag, New York, 291 pp. 


\section{CHAPTER 2 \\ On the role of topography \\ in the wind-driven circulation: \\ the inviscid limit}

\section{Summary}

The ideas developed by Rhines and Young (1982a, b) are used to analyze the effect of topography in simple baroclinic models. The presence of longitude dependent topography induces strong internal jets with transports of the same magnitude as the interior flow. It is shown that the existence of these features is independent of the forcing structure at the top of the model ocean, of the topography form and of the forcing in subsurface layers as long as the latter is small. Some examples are given both for forcings which, in the absence of topography, would give circulations closed in the interior and for forcings that require a western boundary current.

Topography also shifts the line of zero transport allowing for significant flow across the line of zero wind stress curl. Moreover the lines dividing the subtropical gyre from the subpolar gyre are different in every layer, a feature absent in the flat bottom case. 


\subsection{Introduction}

In recent years a number of theories of the ocean general circulation have been developed using conservative (quasi) geostrophic dynamics in the interior region of the domain under consideration. Young and Rhines (1982) (YR) and Rhines and Young (1982a) (RYa), using a quasi-geostrophic, large scale layer model, have shown how the circulation forced by a prescribed Ekman pumping can extend downward through small vertical transfer of horizontal momentum by eddies. Luyten, Pedlosky and Stommel (1983) (LPS) proposed a rather different mechanism, in which the subsurface waters are set in motion by ventilation, through outcrop of density surfaces. All of these models are constrained by the Sverdrup relation between the vertically integrated meridional transport and the wind stress curl. This relation, though, is valid only over a flat bottom, or where the bottom velocity is negligible, and Luyten, Stommel and Wunsch (1985) have shown that in the North Atlantic subpolar gyre, topography has a strong influence on the meridional transport. The purpose of this study is to analyze the effect of large scale topography on the wind-driven circulation. We will borrow directly the ideas and terminology introduced in YR and RYa. We find that large scale topography, when containing a longitudinal variation, can have a dramatic effect on the deduced circulation patterns in models of wind-driven, stratified ocean flows. First, we show that even the presence of a uniform slope in the $x$ (longitudinal) direction alters the qualitative structure of the Sverdrup problem. Closed geostrophic contours in the lower layer now contain flows which can be matched to the Sverdrup external field only with the intervention of internal boundary currents. We show that this arises whether or not the flow within the girdling geostrophic contour has uniform potential vorticity and thus our results do not depend sensitively on the homogenization arguments of RYa. We then demonstrate that ridge-like topography, which we think of as a model of mid-ocean ridge topography, will also produce boundary currents embedded in the Sverdrup interior. This phenomenon is fundamentally different from the first 
case as it depends on strong variations in east-west bottom slope and, in distinction with the previous case, is present in homogeneous models as well. During the preparation of this manuscript, the work of de Szoeke (1985) came to our attention. de Szoeke has considered the role of uniform east-west slope (our first topic). Although there are many elements in common in the two treatments, the directions taken are quite distinct. We have chosen to emphasize the need to embed the circulation in the dynamics appropriate to an oceanic basin. Therefore, in distinction to de Szoeke we do not accept any deep flow on isolines of potential vorticity which intersect the eastern boundary. Nor do we artificially adjust the wind field to produce closure. This has important consequences for the predicted patterns. Our proposed deep flows vanish completely outside the outermost closed geostrophic contour. We also show that the general character of the flow is independent of homogenization of potential vorticity and thus we do not restrict attention to harmonic topography as de Szoeke does. We wish to emphasize that internal jets form with transport of order 1 both in the interior and at the boundary of the region where the subsurface waters move and such a region is well inside the interior of the basin. Part of the motivation for considering topography was to examine whether the effects of topography allow geostrophic flow across the line of zero Ekman pumping. We show that this indeed occurs, especially in the case of forcing of realistic amplitude. Moreover, the lines of zero transport are different in each layer, unlike the flat bottom models. In section 2.2 we present the formulation and solution of the quasi-geostrophic two-layer model for the case of constant bottom slope. That is, analogously to YR and RYa we will force the upper layer with an Ekman pumping, while subsurface forcing, provided by smaller scale activity will be considered small. Thus subsurface flow will be significantly different from zero only in regions where flow streamlines do not encounter boundaries unable to support boundary layers or, obviously, where they close in the interior. The results illustrated by RYa strictly apply to circulations that close in the interior of the ocean although 
eddy resolving general circulation models (see Holland et al., 1984) suggest that they may apply also to flows passing through boundary currents. Therefore we will develop examples with wind stress curl distributions that give circulations which close either in the interior or in the western boundary. In section 2.3 we present the results of calculations of the three-layer model and the continuous stratification case is solved in section 2.4. In section 2.5 the homogeneous and two-layer models are considered for the ridge-like topography. Finally, in section 2.6 we make some speculations on the characteristic transport expected in the internal jets we have predicted. The effects of relative vorticity and of finite viscosity are addressed in the next chapter.

\subsection{Two-layer model}

Our analysis is based on the quasi-geostrophic, two-layer model on a $\beta$-plane, with a wind stress forcing at the top of the model ocean. In this section we consider a simple constant slope topography at the bottom of the lower layer. We will assume that the dominant internal nonconservative mechanism is lateral diffusion of potential vorticity (for a discussion of this choice see Rhines and Young (1982b)). If relative vorticity is neglected lateral diffusion of potential vorticity is equal and opposite in the two layers and therefore gives no contribution to the vertically integrated flow. Therefore bottom friction (for example) is also needed in order to balance the overall input of vorticity but we will assume its effect to be negligibly small except in narrow regions.

The steady two-layer quasi-geostrophic equations are:

$$
\begin{aligned}
& J\left(\psi_{1}, q_{1}\right)=\frac{f_{0} w_{e}}{H_{1}}+\kappa \nabla^{2} q_{1} \\
& J\left(\psi_{2}, q_{2}\right)=\kappa \nabla^{2} q_{2}-\delta \nabla^{2} \psi_{2}
\end{aligned}
$$


with $\delta \ll \kappa f_{0}^{2} /\left(g^{\prime} H_{2}\right) \ll \beta L$, and

$$
\begin{aligned}
& q_{1}=\beta y+\nabla^{2} \psi_{1}+F_{1}\left(\psi_{2}-\psi_{1}\right) \\
& q_{2}=\beta y+\nabla^{2} \psi_{2}+F_{2}\left(\psi_{1}-\psi_{2}\right)+\frac{f_{0} h}{H_{2}} \\
& g^{\prime}=\frac{\rho_{2}-\rho_{1}}{\rho_{2}} g
\end{aligned}
$$

where $H_{1}, H_{2}$ are the average depths of the upper and lower layers respectively and $h$ is the topography. $F_{1}=f_{0}^{2} /\left(g^{\prime} H_{1}\right)$ is the inverse of the Rossby deformation radius squared and $F_{2}=F_{1} H_{1} / H_{2}$.

In the interior of a wind driven gyre the relative vorticity is negligible with respect to the planetary vorticity and the vortex stretching term, and so are all the nonconservative terms. If the barotropic transport equation is formed one obtains:

$$
\beta\left(H_{1}+H_{2}\right) \psi_{b x}=f_{0} h_{x} \psi_{2 y}-f_{0} h_{y} \psi_{2 x}+f_{0} w_{e}
$$

where

$$
\left(H_{1}+H_{2}\right) \psi_{b} \equiv H_{1} \psi_{1}+H_{2} \psi_{2}
$$

Unlike the cases analyzed by RYa and YR, when topography is present the barotropic transport cannot be calculated without solving for the lower layer flow. On the other hand, flow in the lower layer will be (to the order where relative vorticity and nonconservative terms can be neglected) along the contours of

$$
\hat{q}_{2}=\beta y+f_{0} h / H_{2}+F_{2}\left(1+H_{2} / H_{1}\right) \psi_{b}
$$

i. e. $\psi_{2}=\mathcal{F}\left(\hat{q}_{2}\right)$ and in particular $\mathcal{F}\left(\beta y+f_{0} h / H_{2}\right)$ at the boundaries. In general the argument of the right hand side will not be constant on the boundaries and the only solution is the trivial one $\mathcal{F}\left(\hat{q}_{2}\right)=0$. This reasoning cannot be applied when the flow lines close either in the interior or in some boundary layer region, which we choose according to the dynamics of the specific problem. For example if topography is taken to be a constant, say positive, east-west slope $\left(h=h_{x}\left(x-x_{e}\right)\right)$ we know that linear 
frictional boundary layers will be allowed on the northern side of the basin, in addition to the western one. In this case $\psi_{2}$ need not be zero if contours of $\hat{q}_{2}$ hit only the western or the northern boundaries, or obviously if they close upon themselves.

Throughout this section topography has in fact been chosen as a constant positive east-west slope, a ridge-like topography is dealt with in section 2.5. If such a slope is chosen to be of the same order as the $\beta$ term, $\hat{q}_{2}$ contours would go across the basin in diagonal straight lines were it not for the interface displacement due to motion in the upper forced layer. If this forcing is weak, though, such modifications will be small and we can foresee that the lower layer flow will be zero. On the other hand if such an interface displacement becomes comparable to the $\beta$-effect (or the topographic term) there may be some regions where $\hat{q}_{2}$ contours are bent enough to allow flow in the lower layer.

To keep the mathematics as simple as possible we will select the forcing function in the form of (see Fig. 2.1)

$$
\begin{aligned}
w_{e} & =W f(y) \quad(W \leq 0) \\
f(y) & = \begin{cases}y / y_{0} & \text { if } y \leq y_{0} \\
2-y / y_{0} & \text { if } y_{0} \leq y \leq 3 y_{0} \\
y / y_{0}-4 & \text { if } 3 y_{0} \leq y \leq 4 y_{0}\end{cases}
\end{aligned}
$$

which in the absence of topography would give two gyres antisymmetric about the latitude of zero Ekman pumping located at $y=2 y_{0}$.

Wherever the lower layer is motionless the vertically integrated transport can be calculated as

$$
\left(H_{1}+H_{2}\right) \psi_{b}=H_{1} \psi_{1}=f_{0} w_{e}(y)\left(x-x_{e}\right) / \beta
$$

The resulting $\hat{q}_{2}$ contours are

$$
\hat{q}_{2}=\beta y+\left(x-x_{e}\right)\left(f_{0} h_{x} / H_{2}+F_{2} f_{0} w_{e}(y) /\left(H_{1} \beta\right)\right)
$$




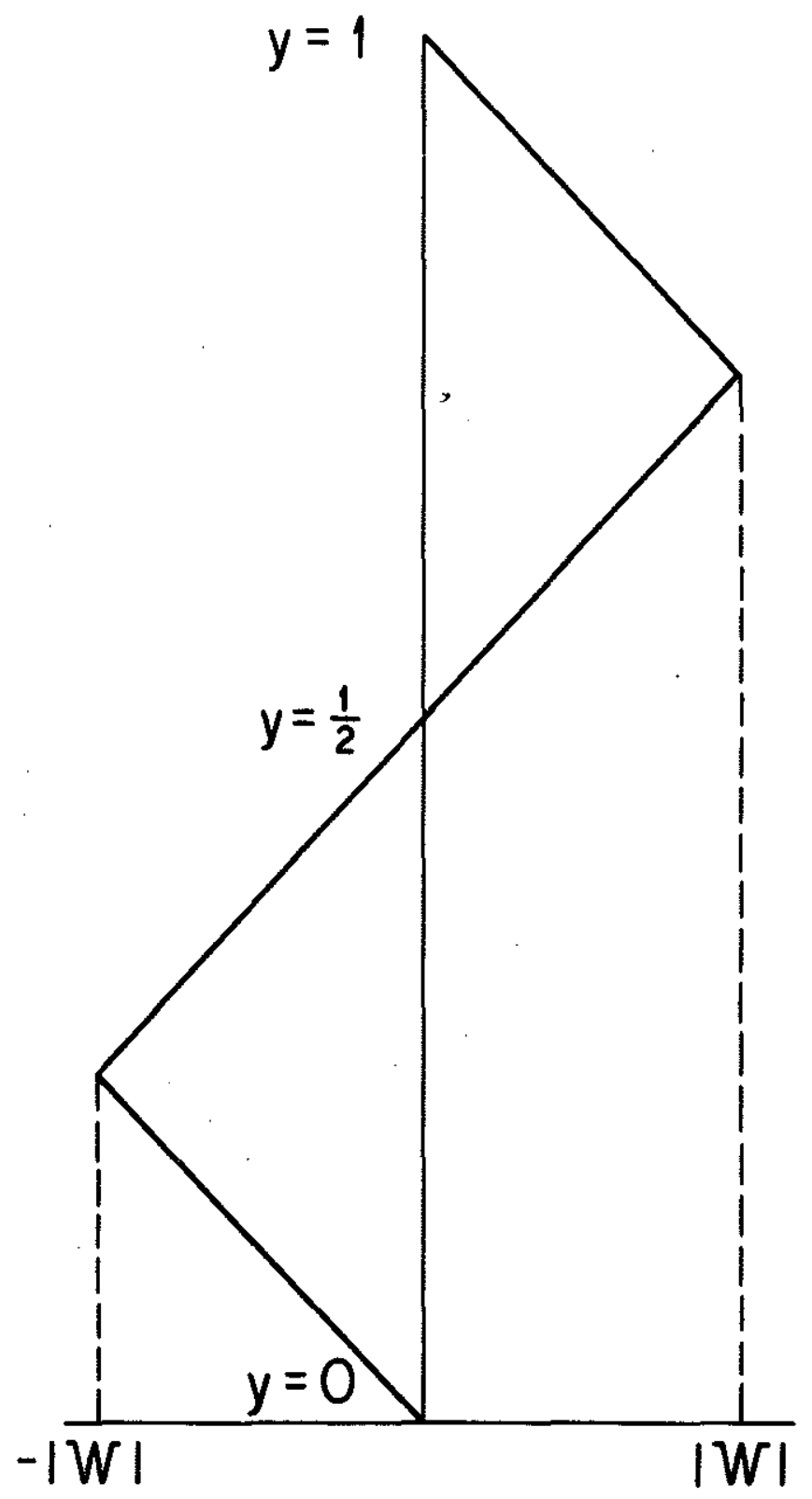

Fig. 2.1. The Ekman pumping (2.2.4) as a function of latitude. The ordinate is $y / 4 y_{0}$. 
Some examples are shown in Fig. 2.2 for different values of the forcing and of the topographic slope. Notice that there is a region where the isolines do not meet either the eastern or the southern boundaries (it is there that the lower layer will be moving) and that this region increases as the forcing gets stronger. Compared with the corresponding case for flat bottom it is evident that the line dividing the subtropical and subpolar gyre is greatly displaced from the line of zero Ekman pumping, being altogether absent for large bottom slope/forcing ratios. Analogously to YR, RYa and Pedlosky and Young (1983), we will assume that where the lower layer moves it will do so as to keep its potential vorticity constant. The arguments leading to the homogenization of potential vorticity may not hold when the flow lines pass through a viscous boundary layer (see Ierley and Young (1983)); they apply though when the flow lines close in the interior. Our point, however, is not to discuss here the validity of the homogenization arguments, and we hope to be able to convince the reader that our results are independent of the choice of constant potential vorticity, although some details may change if a different choice is made.

In order to get closed $\hat{q}_{2}$ contours we must have:

$$
x_{e} f_{0} F_{2}|W| / H_{1}>\beta^{2} y_{0}
$$

Typical oceanic values are: $x_{e}=6000 \mathrm{~km}, f_{0}=10^{-4} \mathrm{sec}^{-1}, H_{1}=1000 \mathrm{~m}, H_{2}=$ $3000 \mathrm{~m}, \beta=10^{-13} \mathrm{~cm}^{-1} \mathrm{sec}^{-1}, y_{0}=1000 \mathrm{~km}, g^{\prime}=1 \mathrm{~cm} / \mathrm{sec}, W=10^{-4} \mathrm{~cm} / \mathrm{sec}$. For these values the ratio of the left hand side to the right hand side of Eq. 2.2.5 is 2 .

Furthermore, in order to obtain an anticyclonic circulation (beside the cyclonic one) as shown in Fig. 2.2c we must have:

$$
x_{e} F_{1}|W|>\beta h_{x} y_{0}
$$



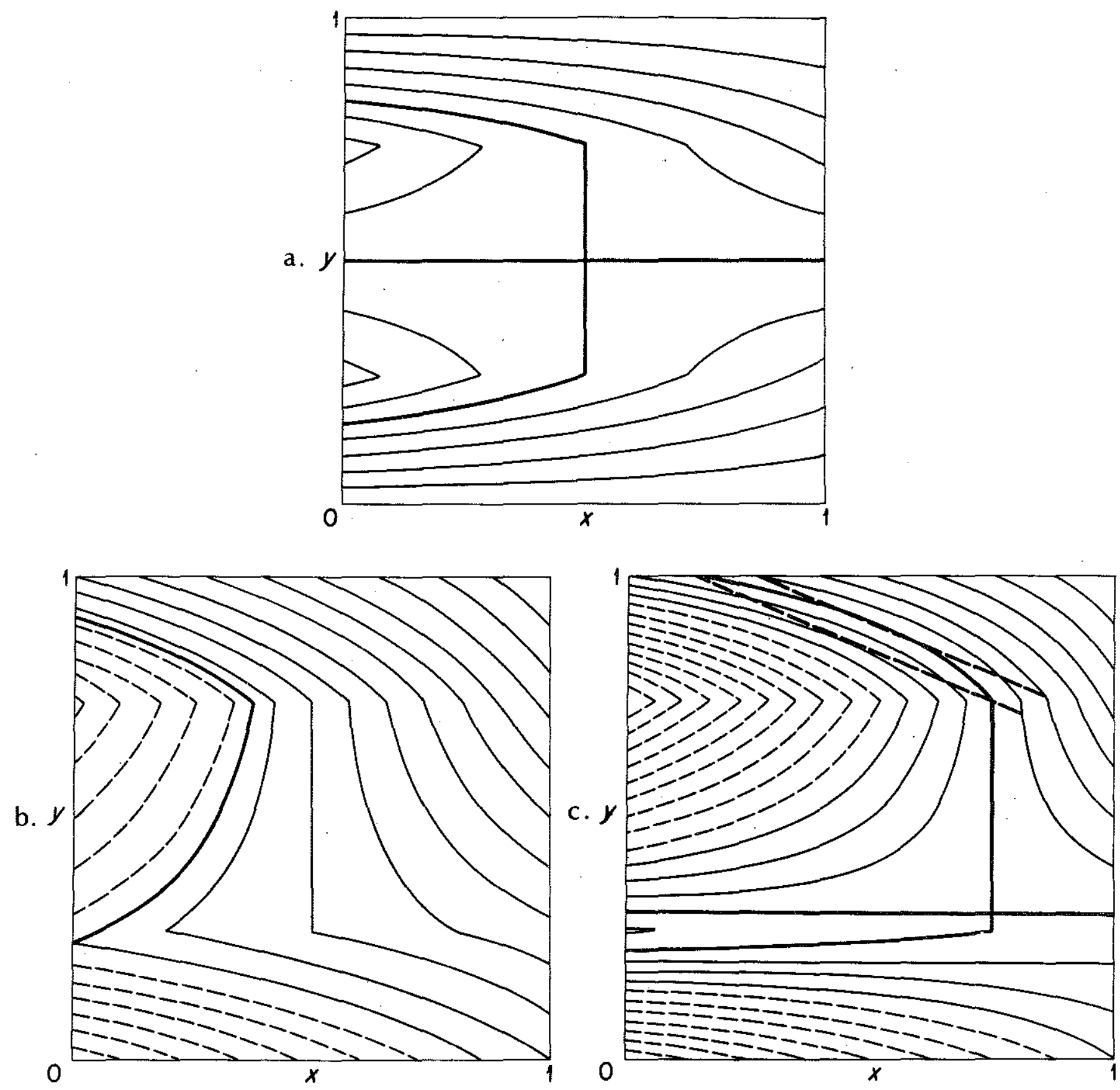

Fig. 2.2. Isolines of $\hat{q}_{2}$ for the two layer model in the nondimensional $x-y$ plane. Both $x$ and $y$ are scaled by $x_{e}$. a) $H_{1}=H_{2}, f_{0} h_{x} /\left(\beta H_{2}\right)=0, y_{0} / x_{e}=1 / 4, f_{0} W F_{2} /\left(\beta^{2} H_{1}\right)=$ 㬝 -0.5 . b) Same as for a) except for $f_{0} h_{x} /\left(\beta H_{2}\right)=0.8$. c) Same as for b) except for $f_{0} W F_{2} /\left(\beta^{2} H_{1}\right)=-1.2$.The heavy line is the outermost closed $\hat{q}_{2}$ contour and the straight dashed lines are the characteristics (2.2.6). 
The constant value $Q_{2}$ of potential vorticity is chosen in such a way as to match the streamfunction $\psi_{2}$ on the outermost closed $\hat{q}_{2}$ contour.

$$
Q_{2}=\beta y+f_{0}\left(h_{x} / H_{2}\right)\left(x-x_{e}\right)+F_{2}\left(\psi_{1}-\psi_{2}\right)
$$

Using this relation to eliminate $\psi_{1}$, the barotropic transport equation becomes

$$
\beta\left(H_{1}+H_{2}\right) \psi_{2 x}-f_{0} h_{x} \psi_{2 y}=f_{0} w_{e}+\beta f_{0} h_{x} / F_{1}
$$

and is independent of the (constant) value of $Q_{2}$, which appears only in the boundary conditions. If the streamfunction is nondimensionalized with

$$
\psi_{2}=\left(\beta x_{e} / F_{1}\right) \psi_{2}^{\prime} \quad \text { and } \quad(x, y)=x_{e}\left(x^{\prime}, y^{\prime}\right)
$$

dropping the primes one gets (see also de Szoeke (1985)):

$$
\left(1+H_{2} / H_{1}\right) \psi_{2 x}-\frac{f_{0} h_{x}}{\beta H_{1}} \psi_{2 y}=\frac{F_{1} f_{0} w_{e}}{\beta^{2} H_{1}}+\frac{f_{0} h_{x}}{\beta H_{1}}
$$

with $\psi_{2}=0$ on $\hat{g}_{2}(x, y)=Q_{2}$ which is a partial differential equation whose characteristics are:

$$
\xi=y+\frac{f_{0} h_{x}}{\beta\left(H_{1}+H_{2}\right)}\left(x-x_{e}\right)
$$

In Fig. $2.2 \mathrm{c}$ it is shown that the characteristics intersect twice the outermost closed $\hat{q}_{2}$ contour in a significant portion of its perimeter. If the choice is made of satisfying the boundary condition of no normal flow at the intersection lying at lower latitudes $\psi$ will have, in general, a value different from zero at the intersection at higher latitudes.

The physical mechanism may be more easily understood if a simpler geometry is analysed. Consider the following forcing function (compare with RYa)

$$
w_{e}= \begin{cases}-\alpha x & \text { if } x^{2}+y^{2}<r_{0}^{2} \\ 0 & \text { if } x^{2}+y^{2}<r_{0}^{2}\end{cases}
$$


with the domain of the basin being $-\infty<x<+\infty$. The governing equations are (2.2.1) and (2.2.2). Where the lower layer is motionless (take $H_{1}=H_{2}=H$ ) the upper layer streamfunction is given by

$$
\begin{array}{ll}
\beta \psi_{1}=\frac{-f_{0} \alpha}{2 H}\left(x^{2}+y^{2}-r_{0}^{2}\right) & \text { if } \quad\left(x^{2}+y^{2}\right)<r_{0}^{2} \\
\beta \psi_{1}=0 & \text { if } \quad\left(x^{2}+y^{2}\right)>r_{0}^{2}
\end{array}
$$

which is the equation of concentric circles centered at $(0,0)$ of maximum radius $r_{0} . \hat{q}_{2}$ contours then become

$$
\begin{array}{ll}
\hat{q}_{2}=\beta y-F_{1} \alpha /(2 H \beta)\left(x^{2}+y^{2}-r_{0}^{2}\right) & \text { if } \quad\left(x^{2}+y^{2}\right)<r_{0}^{2} \\
\hat{q}_{2}=\beta y+\frac{f_{0} h_{x} x}{H} & \text { if } \quad\left(x^{2}+y^{2}\right)>r_{0}^{2}
\end{array}
$$

The first is an equation for arcs of circle. If the forcing is strong enough, i.e. $r_{0}^{2}>x_{0}^{2}+y_{0}^{2}$ with $x_{0}=\beta h_{x} /\left(\alpha F_{1}\right)$ and $y_{0}=\beta^{2} H /\left(\alpha f_{0} F_{1}\right)$, these arcs may close to full circles centered at $\left(x_{0}, y_{0}\right)$ with maximum radius $r_{1}=r_{0}-\sqrt{x_{0}^{2}+y_{0}^{2}}$ (see Fig. 2.3). Notice that in the absence of topography, $x_{0}=0$ and the circles are centered about $x=0$ so that $\iint_{A} w_{e} d a=0$, where $A$ is the area enclosed by any circle centered at $x=0, y=y_{0}$. When topography is added this center is shifted toward one side of the basin so that, as it will be shown in the following, the total input of vorticity is non zero and cannot be balanced without appending boundary layers. If the lower layer potential vorticity is assumed constant inside these circles the total transport is governed by:

$$
2 \beta \psi_{2 x}-f_{0} h_{x} \psi_{2 y} / H=-f_{0} \alpha x / H+\beta f_{0} h_{x} /\left(F_{1} H\right)
$$

where the characteristics are the same as in Eq. 2.2.6. Again on the northwest half of the $r_{1}$ circle the value of $\psi_{2}$ as resulting from the interior dynamics will be different from zero. In the following we show that the mismatch does not depend on the choice of constant potential vorticity and would arise in any case as long as topography depends on $x$. (This result was proved in conjunction with J. Pedlosky.) 


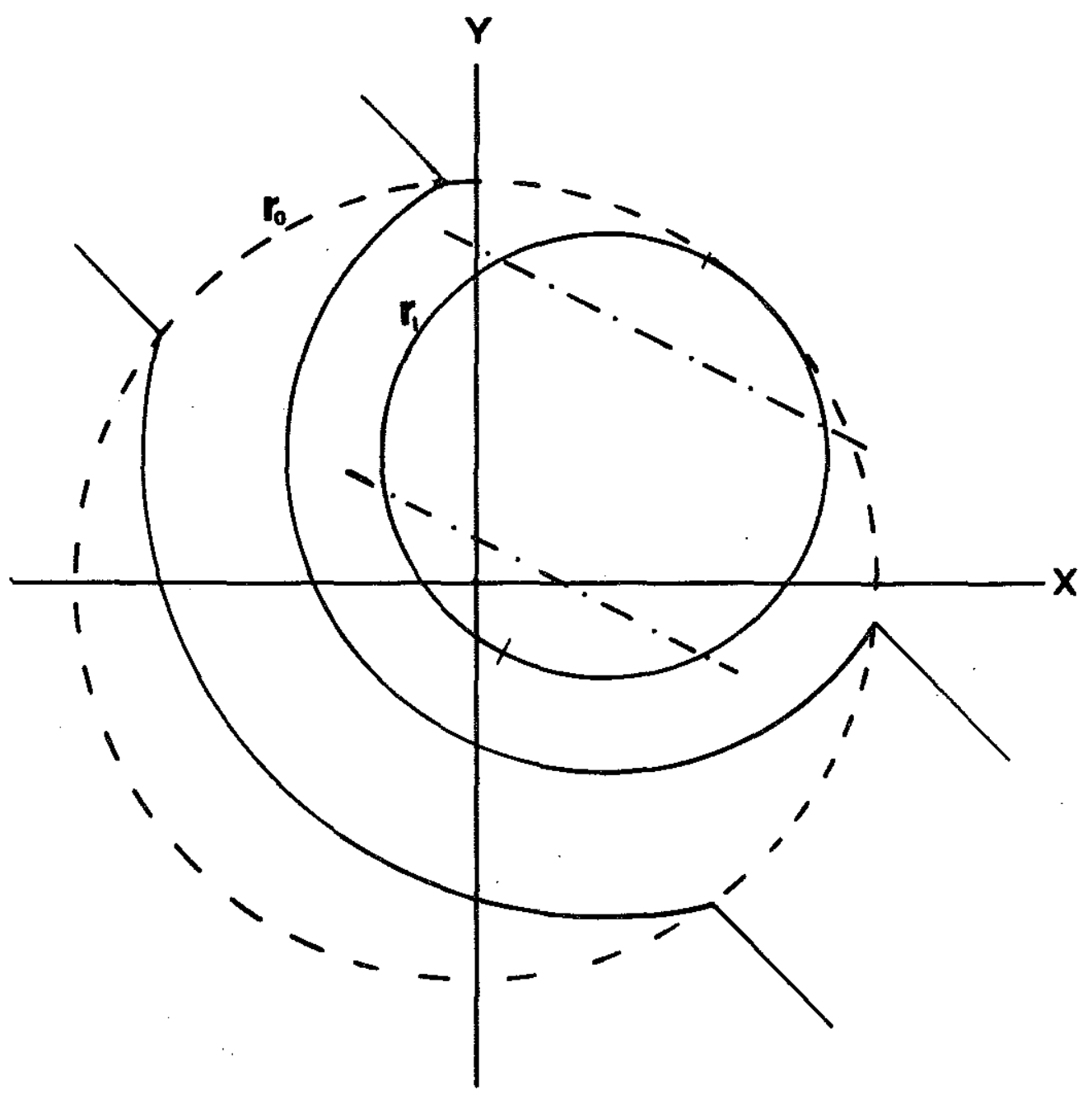

Fig. 2.3. Contours of $\hat{q}_{2}$. The dashed circle $\left(r=r_{0}\right)$ is the bounding contour for the barotropic streamfunction. The dashed-dot lines are the characteristics (2.6). On the northwest half of the outermost closed $\hat{q}_{2}$ contour $\left(r=r_{1}\right)$ the value of $\psi_{2}$ will be different from zero. 
Now returning to the more general case, suppose the forcing is such that there is a contour of $\hat{q}_{2}$ which closes in the interior with $\hat{q}_{2}=\beta y+f_{0} h / H_{2}+F \Psi$ ( $\Psi$ is the upper layer streamfunction calculated with no lower layer flow and $F=f_{0}^{2}\left(H_{1}+\right.$ $\left.\left.H_{2}\right) /\left(g^{\prime} H_{1} H_{2}\right)\right)$ : inside this contour $\psi_{2}=G(q)$ where $q=\beta y+f_{0} h / H_{2}+F \psi_{1}$. Therefore the equation for the vertically integrated transport in the presence of topography becomes (compare with Eq. 2.2.3):

$$
\left(\beta+F \frac{H_{2}}{H_{1}} \frac{\mathrm{d} G}{\mathrm{~d} q}\left(\beta+\frac{f_{0} h_{y}}{H_{2}}\right)\right) \psi_{1 x}-F \frac{f_{0} h_{x}}{H_{1}} \frac{\mathrm{d} G}{\mathrm{~d} q} \psi_{1 y}=\frac{f_{0} w_{e}}{H_{1}}=\beta \Psi_{x} .
$$

We can change to a new coordinate system $\xi$ and $\tau$, where $\xi$ are the characteristics of the flow, such that

$$
\begin{gathered}
\left(\beta+F \frac{H_{2}}{H_{1}} \frac{\mathrm{d} G}{\mathrm{~d} q}\left(\beta+\frac{f_{0} h_{y}}{H_{2}}\right)\right) \xi_{x}=F \frac{f_{0} h_{x}}{H_{1}} \frac{\mathrm{d} G}{\mathrm{~d} q} \xi_{y} \\
\text { with } \begin{array}{c}
\frac{\partial x}{\partial \tau}=\beta+F \frac{H_{2}}{H_{1}} \frac{\mathrm{d} G}{\mathrm{~d} q}\left(\beta+\frac{f_{0} h_{y}}{H_{2}}\right) \\
\frac{\partial y}{\partial \tau}=-F \frac{f_{0} h_{x}}{H_{1}} \frac{\mathrm{d} G}{\mathrm{~d} q}
\end{array}
\end{gathered}
$$

so that the equation for $\psi_{1}$ becomes

$$
\frac{\partial \psi_{1}}{\partial \tau}=\beta \Psi_{x}
$$

We now integrate the equation along a line where $\xi$ is constant $\left(\xi=\xi_{0}\right)$ from the point $\tau_{0}$ to the point $\tau_{1}$, where $\tau_{0}$ and $\tau_{1}$ represent the intersections of $\xi=\xi_{0}$ with the outermost closed $\hat{q}_{2}$ contour, as schematically shown in Fig. 2.4. Assuming that at $\tau=\tau_{0} \quad \psi_{1}\left(\tau_{0}, \xi_{0}\right)=\Psi\left(\tau_{0}, \xi_{0}\right)$ we find

$$
\begin{gathered}
\psi_{1}\left(\tau_{1}, \xi_{0}\right)-\Psi_{1}\left(\tau_{0}, \xi_{0}\right)=\beta \int_{\tau_{0}}^{\tau_{1}} d \tau \frac{\partial}{\partial x} \Psi\left(\xi_{0}, \tau\right) \\
\delta \psi_{1}=\psi_{1}\left(\tau_{1}, \xi_{0}\right)-\Psi\left(\tau_{1}, \xi_{0}\right)=\beta \int_{\tau_{0}}^{\tau_{1}} d \tau \frac{\partial}{\partial x} \Psi\left(\xi_{0}, \tau\right)+\Psi\left(\tau_{0}, \xi_{0}\right)-\Psi\left(\tau_{1}, \xi_{0}\right)
\end{gathered}
$$

Using the definition of $\hat{q}_{2}$ we can write

$$
\Psi\left(\tau_{0}, \xi_{0}\right)-\Psi\left(\tau_{1}, \xi_{0}\right)=1 /\left.F \int_{\tau_{0}}^{\tau_{1}} d \tau \frac{\partial}{\partial \tau}\left(\beta y+f_{0} h / H_{2}-\hat{q}_{2}\right)\right|_{\xi=\xi_{0}}
$$


and recalling that $\hat{q}_{2}\left(\tau_{0}, \xi_{0}\right)=\hat{q}_{2}\left(\tau_{1}, \xi_{0}\right)$,

$$
\delta \psi_{1}=\beta \int_{\tau_{0}}^{\tau_{1}} d \tau \frac{\partial}{\partial x} \Psi\left(\xi_{0}, \tau\right)+1 / F \int_{\tau_{0}}^{\tau_{1}} d \tau \frac{\partial}{\partial \tau}\left(\beta y+f_{0} h / H_{2}\right)
$$

and finally using the definition of $\tau$, i. e. using the chain rule for $\partial / \partial \tau$, one finds

$$
\delta \psi_{1}=\beta / F \int_{\tau_{0}}^{\tau_{1}} d \tau \hat{q}_{2 x}\left(\tau, \xi_{0}\right)
$$

The right hand side vanishes for every value of $\xi_{0}$ only if $\tau=$ const. lines coincide with $x=$ const. lines and this can only be if topography is independent of $x$. Similarly the jump in the lower layer is given by (recalling that $\psi_{2}\left(\tau_{0}, \xi_{0}\right)=0$ )

$$
\delta \psi_{2}=\psi_{2}\left(\tau_{1}, \xi_{0}\right)=\beta \int_{\tau_{0}}^{\tau_{1}} d \tau \frac{\mathrm{d} G}{\mathrm{~d} q} \hat{q}_{2 x}
$$

Therefore, no matter what $G(q)$ will be, a mismatch in the streamfunction field will occur at the boundary of the region where the lower layer is moving. Because the existence of the mismatch is independent of the particular form of $G(q)$ we will continue our calculations making the convenient assumption that potential vorticity is constant in the lower layer. Notice that the jump in the lower layer potential vorticity $q_{2}$ is

$$
q_{2}\left(\tau_{1}, \xi_{0}\right)-\hat{q}_{2}\left(\tau_{1}, \xi_{0}\right)=\beta \int_{\tau_{0}}^{\tau_{1}} d \tau\left(1-F \frac{\mathrm{d} G}{\mathrm{~d} q}\right) \hat{q}_{2 x}
$$

and for the choice $q_{2}=$ constant, we require $d G / d q=1 / F$ so that there is no jump in potential vorticity.

Because of the choice of constant potential vorticity the streamfunction mismatch at the boundary of the outermost closed $\hat{q}_{2}$ contour is equal in the two layers. This is plausible since this choice implies strong interfacial friction which tightly locks the two layers. If for example bottom friction is considered of the same order as interfacial friction, the circulation integral inside closed $\hat{q}_{2}$ contours (see RYa) gives

$$
q=(F+\delta / \kappa) \psi_{2}+\text { constant }
$$




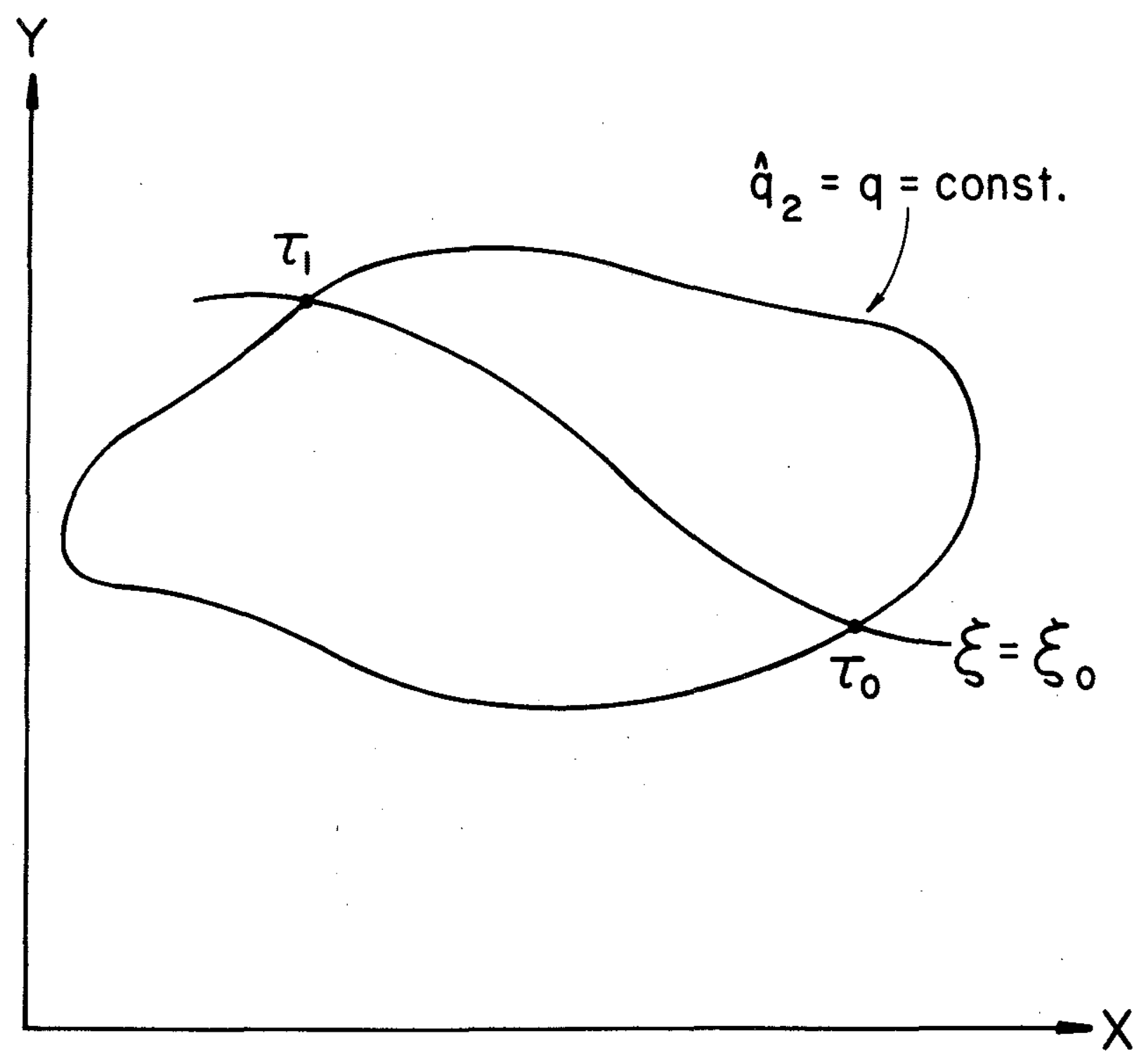

Fig. 2.4. Sketch of the geometry for the integral constraints. See text for explanations. 
where $\delta$ is the coefficient of bottom friction. Now the boundary layer transport in the upper layer will be larger than that in the lower layer, in particular

$$
\delta \psi_{1}=(1+\delta /(\kappa F)) \delta \psi_{2}
$$

In Fig. 2.5 the resulting streamlines for the two layers are shown for the more realistic wind stress pattern (2.2.4). The position of the mismatch where a boundary layer has to be appended is consistent with the notion that in the presence of this topographic slope a barotropic flow would be able to form a linear viscous boundary layer on the northern side of the basin (see Appendix A2). The length of the mismatch region increases with the topographic slope and the strength of the forcing (see Table 2.1a). From Eq. A2.3 it can be checked that as the point of maximum upwelling shifts northward the length of the mismatch region becomes larger. On the other hand if one considers a fixed distance on the outermost closed $\hat{q}_{2}$ contour from the point $x_{0}$ where the discontinuity begins, the strength of the jump is almost independent of the parameters. Because the jump increases with increasing distance from $x$ (see Table 2.1b), the final transport entering the western boundary will increase with the topographic slope and the forcing strength.

It is appropriate to mention here that the line of zero transport does not coincide in the two layers unlike the case for flat bottom. Moreover there is some vertically integrated flow across the line of zero Ekman pumping. When a subtropical gyre appears in the lower layer (see Fig. $2.5 \mathrm{~b}$ ) the flow tends to be rather zonal in the southwest corner of the subpolar gyre, in close analogy to the case of a barotropic flow in the presence of the same topography (see Appendix B2 and Fig. B2). As could have been expected, when the forcing gets stronger the circulation in the lower layer resembles more closely the forcing pattern itself and, therefore, the flat bottom circulation. Comparing the lower layer streamfunctions of Figs. 2.5a and 2.5b it can 

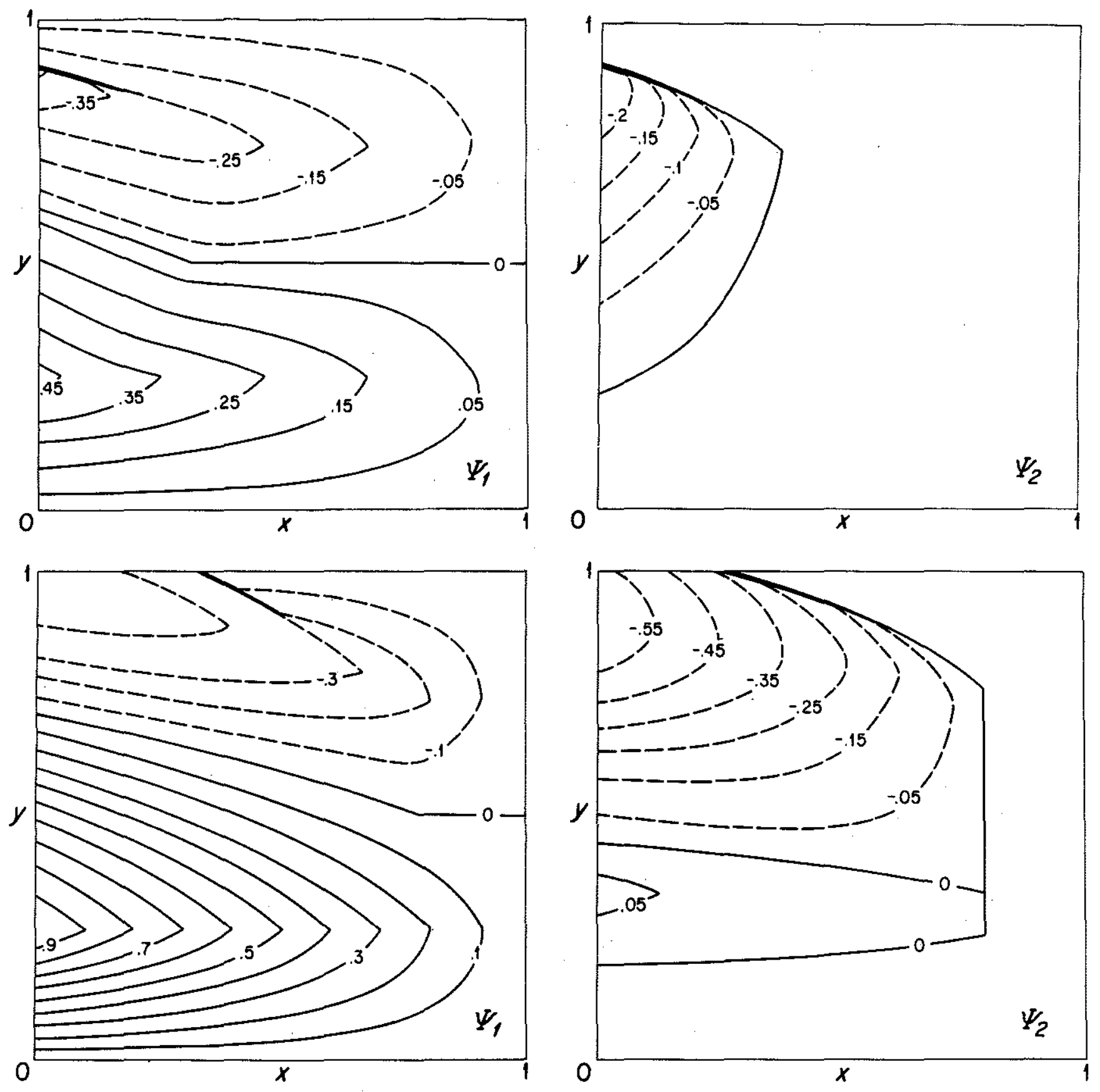

Fig. 2.5. Streamlines for the flow in the two layer model. Top) $f_{0} W F_{2} /\left(\beta^{2} H_{1}\right)=-0.5$ Bottom) $f_{0} W F_{2} /\left(\beta^{2} H_{1}\right)=-1.2$ The line of increasing thickness shows the location of the discontinuity. 


\begin{tabular}{|c|c|c|c|c|c|}
\hline \multirow{2}{*}{ Forcing } & \multicolumn{5}{|c|}{ Topographic slope } \\
\cline { 2 - 6 } & 0.4 & 0.8 & 1.2 & 1.6 & 2.0 \\
\hline-0.50 & 0.000 & 0.154 & 0.193 & 0.213 & 0.225 \\
-0.75 & 0.203 & 0.390 & 0.390 & 0.390 & 0.390 \\
-1.00 & 0.315 & 0.500 & 0.528 & 0.510 & 0.500 \\
-1.25 & 0.388 & 0.560 & 0.628 & 0.604 & 0.584 \\
-1.50 & 0.440 & 0.601 & 0.667 & 0.684 & 0.653 \\
-1.75 & 0.480 & 0.663 & 0.695 & 0.729 & 0.714 \\
-2.00 & 0.513 & 0.657 & 0.717 & 0.750 & 0.780 \\
\hline
\end{tabular}

Table 2.1a: Easternmost point of discontinuity, for the constant slope topography, in units of $x_{e}$ as a function of the nondimensional forcing $f_{0} W F_{1} /\left(\beta^{2} H_{1}\right)$ (rows) and topographic slope $f_{0} h_{x} /\left(\beta H_{2}\right)$ (columns).

\begin{tabular}{|l|l|l|l|l|l|l|l|c|}
\hline & \multicolumn{7}{|c|}{ Distance from $x_{0}$} \\
\cline { 2 - 9 } Forcing & 0.05 & 0.10 & 0.15 & 0.20 & 0.25 & 0.30 & 0.35 & 0.40 \\
\hline-1.25 & -0.03 & -0.07 & -0.11 & -0.16 & -0.21 & -0.26 & -0.32 & -0.38 \\
-1.75 & -0.02 & -0.06 & -0.11 & -0.16 & -0.21 & -0.27 & -0.35 & -0.43 \\
\hline
\end{tabular}

Table 2.1b: Nondimensional transport of the lower layer flow at the northern boundary of closed $\hat{q}_{2}$ contours, as a function of the distance from the point $x_{0} / x_{e}$ where the discontinuity begins (columns), for two values of the forcing $f_{0} W F_{1} /\left(\beta^{2} H_{1}\right)$ (rows), for a fixed value of the topographic slope $f_{0} h_{x} /\left(\beta H_{2}\right)=0.8$.

be seen that the water crossing the zero Ekman pumping line (at $y=1 / 2$ ) is of a more southern origin when the forcing is weaker.

We now want to increase the vertical resolution of our model in order to test the robustness of our results and to see whether topography produces other new features when more layers are added. 


\subsection{Three-layer model}

In order to make useful comparison between the two and three layer model, we have to choose our parameters in such a way that these models represent different approximations of the same physical process. Therefore some minimal requirements arise: the total mass of our system has to be the same and the mass of cold and warm waters have to be separately conserved. The first statement implies that, to the order considered by the Boussinesq approximation the total volume has to be the same in the two models. Therefore, indicating the two-layer model variables with primes

$$
H_{1}+H_{2}+H_{3}=H_{1}^{\prime}+H_{2}^{\prime}=2 H \quad \text { for equal layers }
$$

The second statement is not as easy to quantify, but a simple formulation which clearly satisfies it is:

$$
\rho_{1}^{\prime}=\rho_{1} ; \quad H_{1}^{\prime}=H_{1}=H ; \quad \rho_{2} H_{2}+\rho_{3} H_{3}=\rho_{2}^{\prime} H_{2}^{\prime}
$$

which gives

$$
g^{\prime}\left(H_{2}+H_{3}\right)+g^{n} H_{3}=g_{1}^{\prime} H
$$

where $g^{\prime}=\left(\rho_{2}-\rho_{1}\right) g / \rho_{2}, \quad g^{\prime}=\left(\rho_{3}-\rho_{2}\right) g / \rho_{2}, \quad g_{1}^{\prime}=\left(\rho_{2}^{\prime}-\rho_{1}^{\prime}\right) g / \rho_{1}^{\prime}$.

The equations for a quasi-geostrophic, very large scale, $\beta$-plane, steady three layer model in the presence of wind stress forcing, bottom topography, small lateral diffusion of potential vorticity and very small bottom friction, are:

$$
\begin{aligned}
& J\left(\psi_{1}, q_{1}\right)=\frac{f_{0} w_{e}}{H_{1}}+\kappa \nabla^{2} q_{1} \\
& J\left(\psi_{2}, q_{2}\right)=\kappa \nabla^{2} q_{2} \\
& J\left(\psi_{3}, q_{3}\right)=\kappa \nabla^{2} q_{3}-\delta \nabla^{2} \psi_{3}
\end{aligned}
$$

where 


$$
\begin{aligned}
& q_{1}=\beta y+F_{1}\left(\psi_{2}-\psi_{1}\right) \\
& q_{2}=\beta y+F_{2}\left(\psi_{1}-\psi_{2}\right)+F_{3}\left(\psi_{3}-\psi_{2}\right) \\
& q_{3}=\beta y+F_{4}\left(\psi_{2}-\psi_{3}\right)+\frac{f_{0} h}{H_{3}}
\end{aligned}
$$

where $F_{1}=f_{0}^{2} /\left(g^{\prime} H_{1}\right), F_{2}=f_{0}^{2} /\left(g^{\prime} H_{2}\right), F_{3}=f_{0}^{2} /\left(g^{\prime \prime} H_{2}\right), F_{4}=f_{0}^{2} /\left(g^{\prime \prime} H_{3}\right)$.

Again we will assume that the potential vorticity is constant in the two unforced layers, in those regions where the streamlines do not hit either the eastern or the southern boundaries. The limiting contours of such regions are again found assuming no flow in each layer outside them and are shown in Figs. 2.6a and 2.6b. Now the condition on the forcing strength in order to have a deep moving layer is:

$$
x_{e} F_{1}^{\prime} f_{0}|W| / H>\gamma \beta^{2} y_{0}
$$

with

$$
\gamma=\frac{H_{3} H_{2}}{H^{2}} \frac{g^{\prime}}{g_{1}^{\prime}}+\frac{H_{3}}{H} \frac{\left(g^{\prime}+g^{\prime}\right)}{g_{1}^{\prime}}+\frac{g^{\prime}}{g_{1}^{\prime}} \frac{H_{2}}{H}
$$

and the analogous condition for the middle layer is:

$$
x_{e} F_{2} f_{0}|W| / H>\beta^{2} y_{0}
$$

Notice that, because of our choice (2.3.1), in order for the deepest layer to move the forcing has to be stronger than in the equivalent situation for two layers. In particular

$$
x_{e} F_{1}^{\prime} f_{0}|W| / H>\left(1+H_{3} H_{2} g^{n} /\left(H^{2} g_{1}^{\prime}\right)\right) \beta^{2} y_{0}
$$

which is to be compared with Eq. (2.2.5). In the following we will present the results without showing the detailed derivation, emphasizing the similarities with the two layer model and the new findings for increased vertical resolution. One result of the introduction of bottom topography can be readily seen by inspection of Fig. $2.6 \mathrm{~b}$ : there is a small region at the northwest corner of the basin where the deepest layer 
moves although the middle layer doesn't. This is quite contrary to the results in the absence of topography where the region of "closed $\hat{q}_{3}$ contours is properly contained in the region of closed $\hat{q}_{2}$ contours" (see RYa). This lack of "nesting" of the closed $\hat{q}$ contours also occurs when ventilation is present (see LPS) although for different reasons. In the calculations we have done, though, we haven't corrected the lowest layer flow for the absence of motion in the middle layer, since this region is very small. In the region where all layers are moving, the characteristics of the equation for the vertically integrated transport are the same as in the two-layer model (Eq. 2.2.6). Therefore the characteristic lines are independent of the stratification. In Fig. 2.7 an example of flow is shown for one value of the forcing strength. Notice that, as in the two layer case, the line of zero transport is different in each layer, except, of course, in the region where topography is not felt. Again in all layers there will be noticeable exchange of water across the zero Ekman pumping line, a feature which is absent in the flat bottom model. Although the small region where $\psi_{2}=0$ and $\psi_{3} \neq 0$ is not shown we have checked that the mismatch at the southern side of its limiting contour, where $\psi_{2}$ and $\psi_{3}$ become non zero, still exists together with a non zero flow at the northern rigid boundary. 

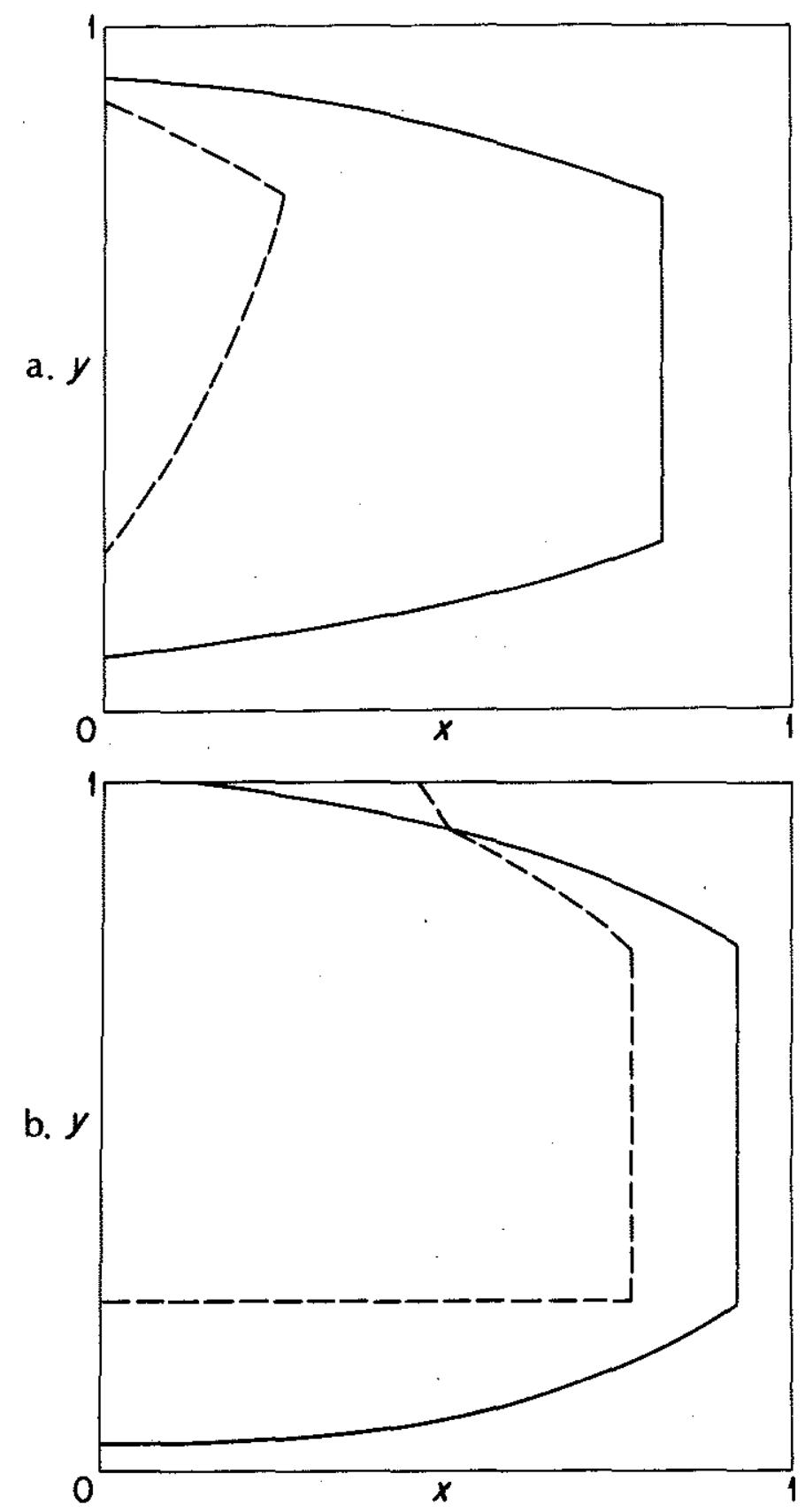

Fig. 2.6. Bounding contours for the regions of flow in the middle and lowest layer. Outside the dashed line $\psi_{3}=0$. Outside the full line $\psi_{2}=0$. a) $f_{0} h_{x} /\left(\beta H_{2}\right)=$ $0.8, y_{0} / x_{e}=1 / 4, H_{1}=H, f_{0} W F_{1} /\left(\beta^{2} H\right)=-0.5, g^{\prime} / g_{1}^{\prime}=3 / 4, g^{n} / g_{1}^{\prime}=$ $1 / 2, H_{2} / H=H_{3} / H=1 / 2$. b) Same as for a) except for $f_{0} W F_{1} /\left(\beta^{2} H\right)=-1.2$ 

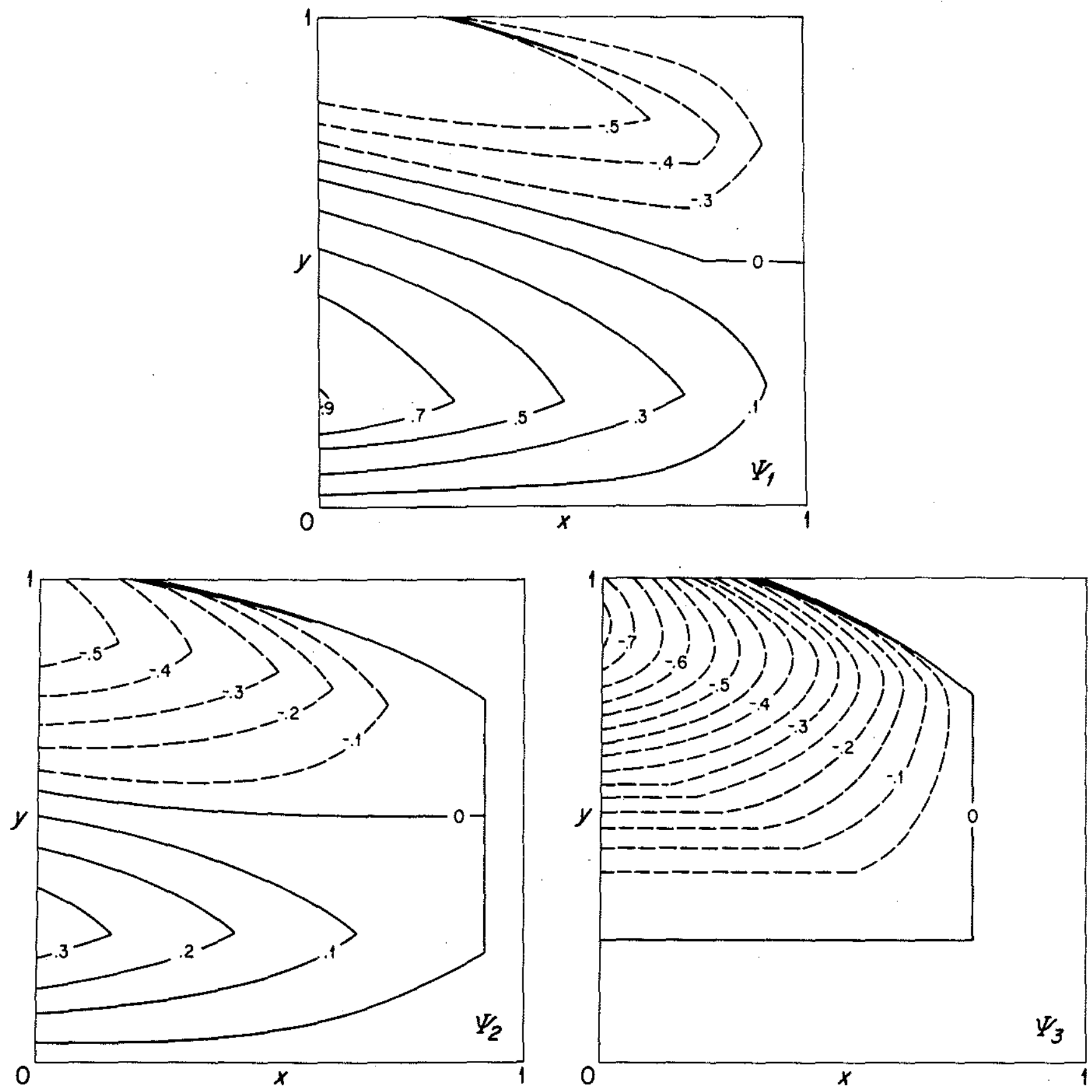

Fig. 2.7. Streamlines for the three layer model. Same parameters as in Fig. 2.6b. The line of increasing thickness shows the location of the discontinuity. 


\subsection{Continuous stratification model}

With continuous stratification the Boussinesq potential vorticity equation is given by

$$
J(\psi, q)=\kappa \nabla^{2} q
$$

with boundary conditions $\quad J\left(\psi, f_{0} \psi_{z} / N^{2}\right)=-w_{e} \quad$ at $\quad z=0$

$$
\text { and } \quad J\left(\psi, f_{0} \psi_{z} / N^{2}+h\right)=0 \quad \text { at } z=-H .
$$

Here $q=\nabla^{2} \psi+\left(f_{0}^{2} \psi_{z} / N^{2}\right)_{z}+\beta y$ and $N$ is the Brunt-Vaisala frequency. All the calculations will be performed under the assumption of constant Brunt-Väisälä frequency. Also in the following we will define the quantity $F=f_{0}^{2} /(N H)^{2}$ which is equivalent to the inverse of the Rossby deformation radius squared in the layer models. With this definition and with the assumption that relative vorticity can be neglected, the expression for potential vorticity becomes:

$$
q=F H^{2} \psi_{z z}+\beta y
$$

Following YR we will assume that the wind driven circulation is contained within a "bowl" between $z=-D(x, y)$ and the surface and that potential vorticity is constant inside the "bowl". In Appendix $\mathrm{C} 2$ it is shown that the value of $q$ inside the bowl is independent of the vertical coordinate as well as of the horizontal coordinates. For bottom topography to be felt by the flow, the circulation must extend to the bottom and the bowl must intersect the floor at $z=-H$. The model geometry in the $y-z$ plane is depicted in Fig. 2.8. The equations governing the flow are then

$$
F H^{2} \psi_{z z}+\beta y=\beta y_{1}
$$

with boundary conditions $\quad J\left(\psi, F H \psi_{z}\right)=-f_{0} w_{e} / H \quad$ at $\quad z=0$ and $\quad \psi=\psi_{z}=0 \quad$ at $z=-D \quad$ where $D<H$ or $J\left(\psi, F H \psi_{z}+f_{0} h / H\right)=0 \quad$ at $\quad z=-H \quad$ where $\quad D>H$ 
where $h$ is the bottom topography and $y_{1}$ is the constant value of potential vorticity.

In the region $D<H$ the bottom topography has no influence and the circulation is the same as in YR

$$
\begin{aligned}
\psi & =\beta\left(y_{1}-y\right)(z+D)^{2} /\left(2 F H^{2}\right) \\
\text { where } \quad D^{3} & =F H^{2} f_{0} 6 w_{e}(y)\left(x-x_{e}\right) / \beta^{2}\left(y_{1}-y\right) .
\end{aligned}
$$

Given the form of $D$ it is clear that $y_{1}$ must coincide with a latitude where the Ekman pumping is zero, otherwise $D$ would become infinite at the latitude $y=y_{1}$. For the Ekman pumping pattern shown in Fig. 2.1 there are three possibilities: $y_{1}=0, y_{1}=$ $2 y_{0}, y_{1}=4 y_{0}$. Contours of $(D / H)^{3}$ are shown in Fig. 2.9 for each of these choices. For the choice $y_{1}=0$ and $y_{1}=4 y_{0}$, the depth of the bowl is not symmetric around the line of zero Ekman pumping $y=2 y_{0}$ as one would expect for symmetric forcing, and becomes negative in half of the basin. For the choice $y_{1}=2 y_{0}$, the lines of constant $D$ are symmetric around the latitude of zero wind stress curl and they resemble the outermost closed $\hat{q}_{2}$ contour for the two layer model shown in Fig. 2.2a. Therefore, from the analogy with the layered model the choice $y=2 y_{0}$ is the correct one. A more rigourous derivation of this result can be found in Appendix C2. With this choice $D$ achieves its maximum on the western boundary at the latitude $y=2 y_{0}$. The maximum depth of the bowl is given by $D=\left(6 W x_{e} f_{0} F H^{2} /\left(\beta^{2} y_{0}\right)\right)^{1 / 3}$. If the values quoted in section 2.2 are used and if $N=10^{-3} \mathrm{sec}^{-1}$, the circulation goes as deep as $6000 \mathrm{~m}$. Before this depth is reached, the bowl containing the circulation will intersect the bottom.

In the region where $D>H$ the bottom boundary condition is $J\left(\psi, F H \psi_{z} /+\right.$ $\left.f_{0} h / H\right)=0$ and is satisfied whenever $\psi=\mathcal{F}\left(F H \psi_{z}+f_{0} h / H\right)$ at $z=-H$. On the boundary of this region (given by $D=H$ ) pressure and density ought to be continuous so $\psi=\psi_{z}=0$. This implies $0=\mathcal{F}(h)$ which in general is a contradiction. Therefore, for all the contours of $\hat{q}=\left.F H \psi_{z}\right|_{z=-H}+f_{0} h / H$ that encounter the boundary $D=H$, the only possible solution is $\psi=0$. Thus there is going to be a region (shaded in Fig. 


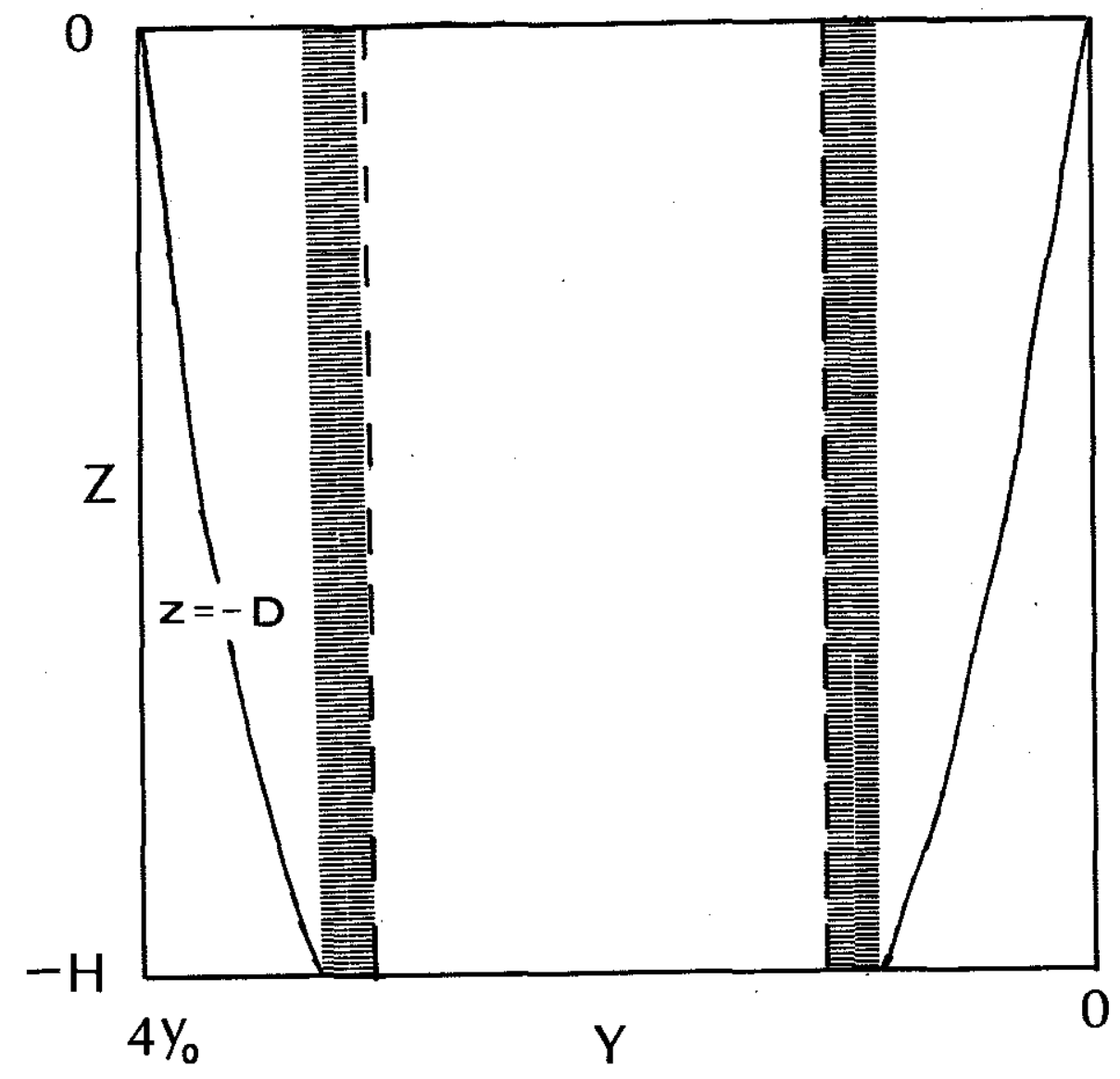

Fig. 2.8. Schematic geometry for the continuously stratified model in the $y-z$ plane. The circulation is contained between the surfaces $z=-D(x, y)$ and $z=0$. The "bowl" intersects the floor at $z=-H$. In the shaded region the bottom water is at rest and topography is not felt. In the innermost region the bottom water moves and topography influences the flow top to bottom. 

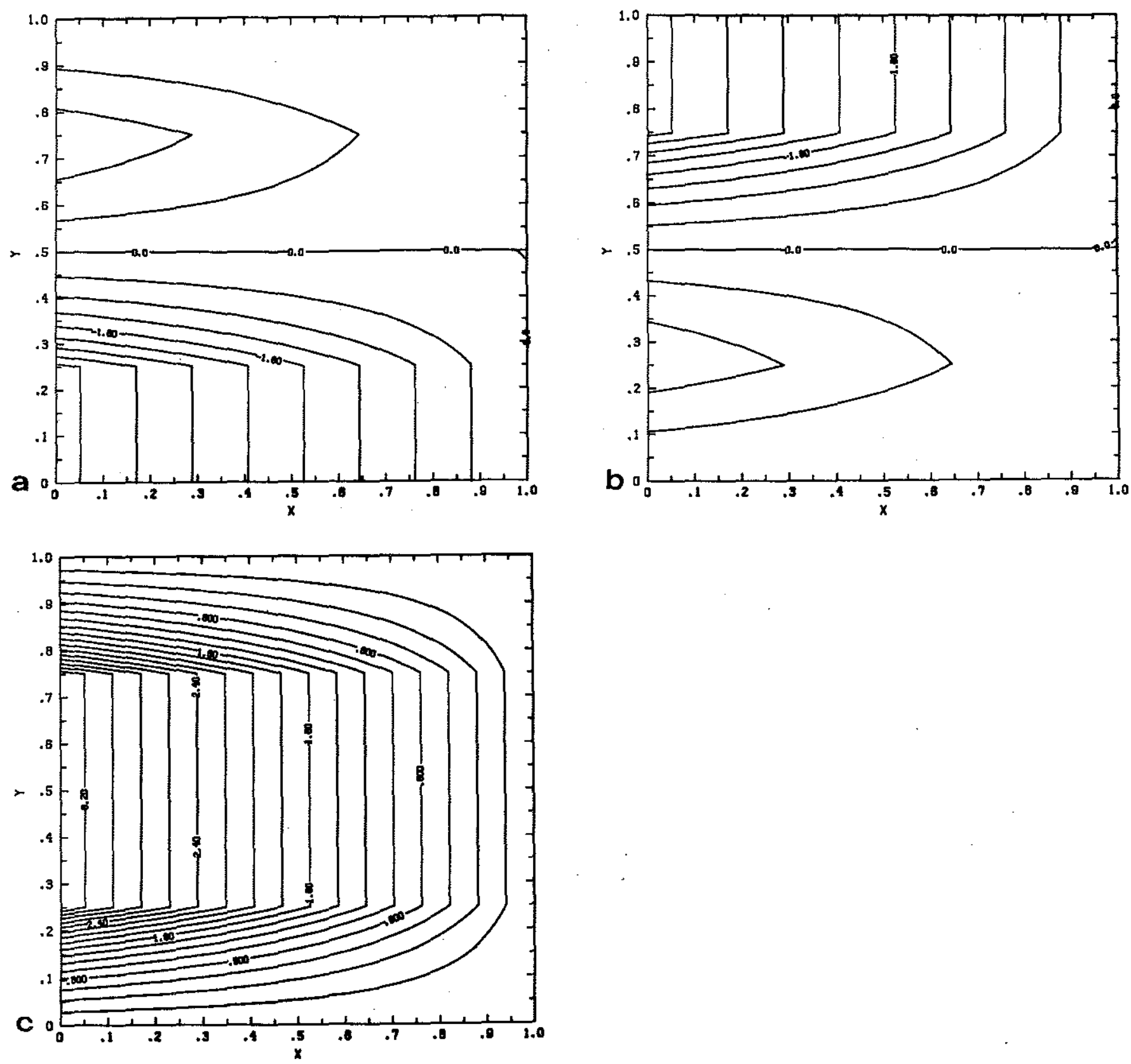

Fig. 2.9. Contours of $D^{3} / H^{3}$ as a function of $y / 4 y_{0}$ and $x / x_{e}$ for different values of the homogenized potential vorticity. a) $y_{1}=0$. In the region south of the zero Ekman pumping latitude $\left(y / 4 y_{0}=0.5\right)$ the contours intersect the southern boundary, $D$ is negative and no circulation can occur. b) $y_{1}=4 y_{0}$. In the region north of the zero Ekman pumping latitude the contours intersect the northern boundary, $D$ is negative and no circulation can occur. c) $y_{1}=2 y_{0} . D$ is symmetric about the latitude of zero Ekman pumping. The maximum value of $D$ occurs on the western boundary at $y=y_{1}$. 
2.8) where, even though the circulation goes all the way to the bottom, the very abyssal water is at rest and topography is not felt. In this region the flow satisfies

$$
F H^{2} \psi_{z z}=\beta\left(y_{1}-y\right)
$$

with vertical boundary conditions

$$
\begin{array}{cl}
F H J\left(\psi, \psi_{z}\right) & =-w_{e} \quad \text { at } \quad z=0 \\
\text { and } \quad \psi & =0 \quad \text { at } \quad z=-H
\end{array}
$$

and horizontal boundary conditions $\left.\quad \psi_{z}\right|_{z=-H}=0$ at $D=H$

The solution is given by

$$
\psi=\beta\left(y_{1}-y\right)\left[(z / H+1)^{2} / 2+(z / H+1)\left(D^{3} / H^{3}-1\right) / 3\right] / F
$$

and both $\psi$ and $\psi_{z}$ are continuous at $D=H$ with the solution given by (2.4.2).

The solution (2.4.3) is valid as long as the $\hat{q}$ contours intersect the curve $D=H$. If $\hat{q}$ contours are closed then the bottom may not be at rest and topography will affect the flow. In Fig. 2.10 contours of $F H \psi_{z}+f_{0} h / H$ (solid lines) are shown for the constant slope topography $h=h_{x} x\left(h_{x}>0\right)$ used previously, the dashed line is the contour $D=H$ and represents the intersection of the bowl containing the circulation with the bottom. As usual we assume that contours will close in appropriate boundary layers on solid walls (in this example the western and northern walls). In order to get closed contours of $\hat{q}$ the forcing has to be strong enough so that

$$
6 F x_{e} f_{0}|w| / H>\beta^{2} y_{0}
$$

which is analogous to the condition obtained for the layer model, Eq. 2.2.5. Similarly to the three layer model, due to the presence of a northern boundary current, there is a region close to the northwest corner where the bottom may move although the water right above it doesn't. This means that, near the northwest corner of the basin the bowl $z=-D(x, y)$ will be folded. In Fig. 2.11a a schematic section of the region of motion in the $y-z$ plane, at a longitude where the folding occurs, is shown. In 


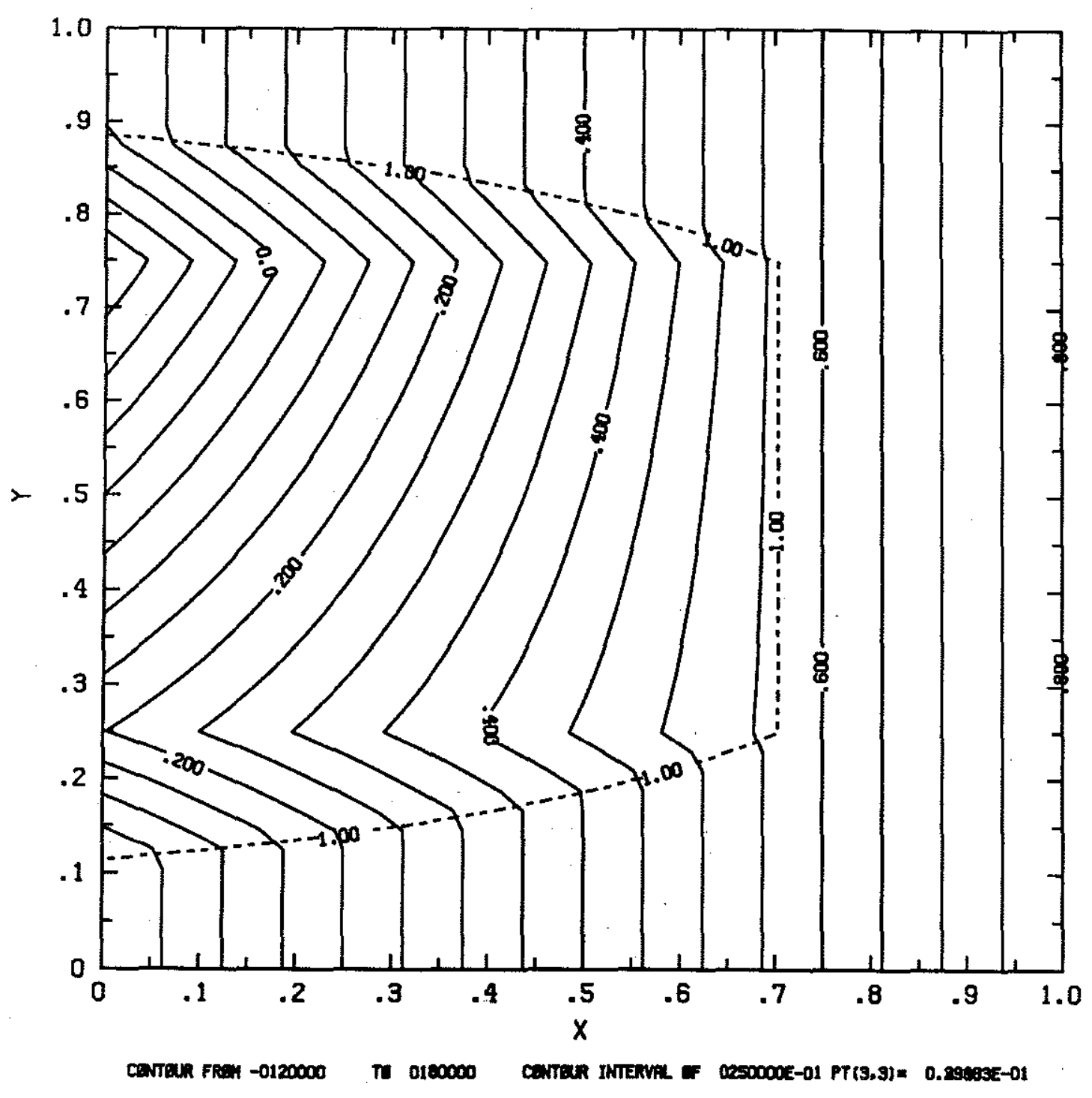

Fig. 2.10. Contours of $\hat{q}=\left.F H \psi_{x}\right|_{s=-H}+f_{0} h / H$, in units of $\beta y_{0}$ (solid lines), computed under the assumption that the water at $z=-H$ is at rest. This assumption is not valid for the region where $\hat{q} / \beta y_{0}<0.2$. In that region the bottom will move keeping $\hat{q}=Q_{0}=0.2 \beta y_{0}$. The dashed line is the contour $D=H$ and represents the intersection of the circulation bowl with the floor. 
Fig. 2.11b the section in the $x-y$ plane, at $z=-H$, is shown. In Fig. 2.11 three regions are distinguished: in region $\mathbf{C}$ the water moves everywhere but is at rest at the bottom and topography has no effect; in region $B$ the motion occurs top to bottom and topography is felt; in region $A$ the water is quiescent at mid depth although it is sandwiched between a moving top and bottom.

Where the water on the bottom moves (regions $A$ and $B$ ), we will assume that $F H \psi_{z}+f_{0} h /\left.H\right|_{z=-H}$ is constant, say equal to $Q_{0}$, since the quantity $\hat{q}$ can be thought of as bottom layer potential vorticity for a layer model, in the limit of vanishing layer thickness. The constant $Q_{0}$ is determined by matching density $\left(\psi_{z}\right)$ at the outermost closed $\hat{q}$ contour.

Let's now compute the circulation in region $A$, where the bowl is folded. Suppose the surface is folded at the level $z=-D^{\prime}(x, y)$. For $z>-D^{\prime}$ (region $A^{\prime}$ in Fig. 2.11a) the governing equations are the same as in (2.4.1) and

$$
\psi=\beta\left(y_{1}-y\right)(z+D)^{2} /\left(2 F H^{2}\right)
$$

In the region $-H<z<-D^{\prime}$ (region $A^{\prime \prime}$ in Fig. 2.11a) we have

$$
F H^{2} \psi_{z z}+\beta y=\beta y_{1}
$$

with boundary conditions $\quad F H \psi_{z}+f_{0} h / H=Q_{0} \quad$ at $\quad z=-H$

$$
\text { and } \quad \psi=\psi_{z}=0 \quad \text { at } \quad z=-D^{\prime}
$$

The solution is given by

$$
\psi=\beta\left(y_{1}-y\right)\left(z+D^{\prime}\right)^{2} /\left(2 F H^{2}\right)
$$

with $D^{\prime}$ given by

$$
D^{\prime}=H+H \frac{Q_{0}-f_{0} h / H}{\beta\left(y_{1}-y\right)}
$$

The expression for the folding curve in the $x-y-z$ space is then given by the intersection of the two surfaces.

$$
\left\{\begin{array}{l}
z=-D(x, y) \\
z=-D^{\prime}(x, y)
\end{array}\right.
$$



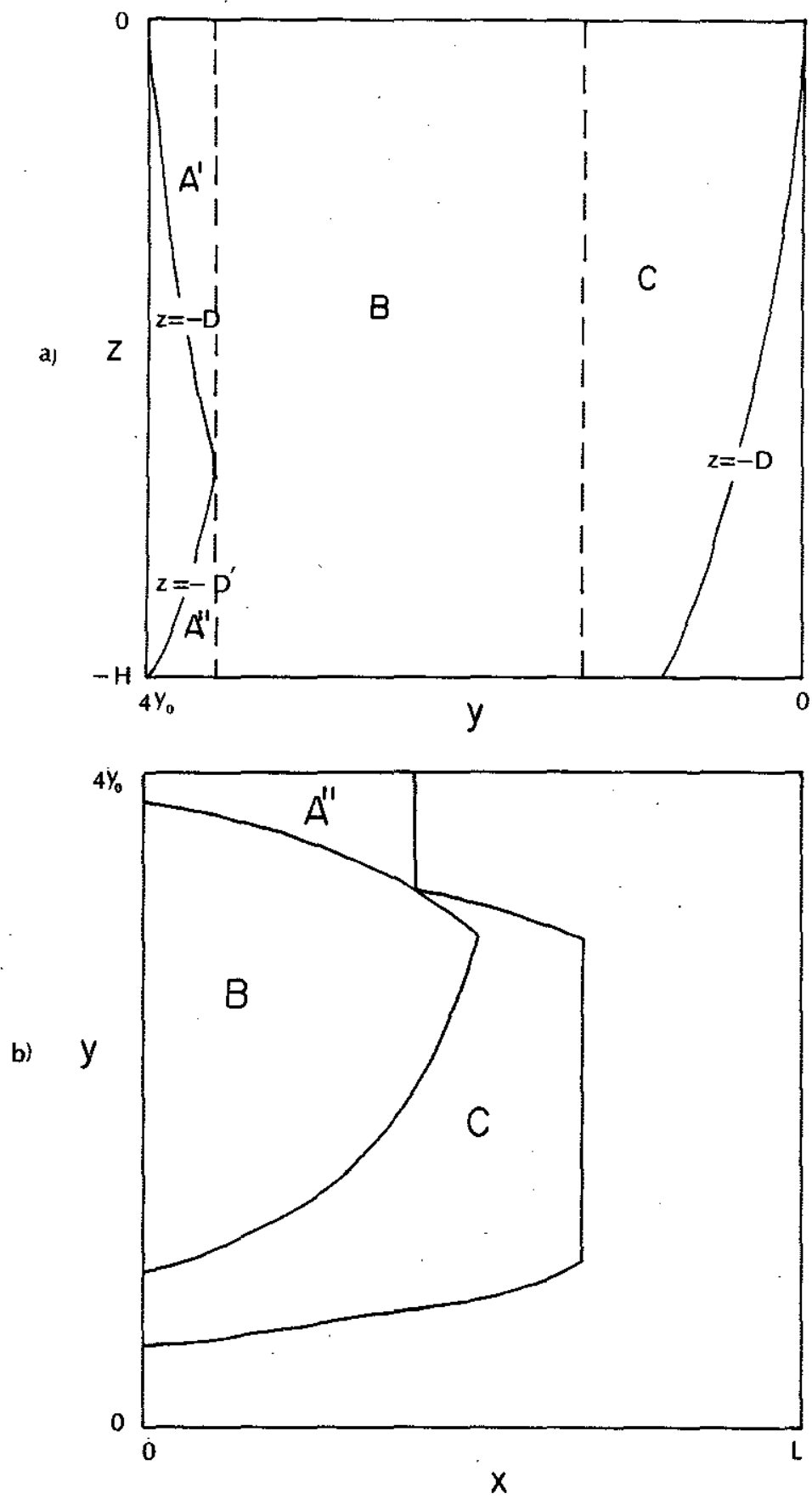

Fig. 2.11. Schematic of the different regions in the $y-z$ and $x-y$ planes. a) Vertical section at a longitude where the folding occurs, say $x / x_{0}=0.1$ in Fig. 2.10. Regions $A$ : the water moves only in regions $A^{\prime}$ and $A^{\prime \prime}$, so that the water at middepth is queiscent. In region $A^{\prime}$ the circulation is confined between $z=-D$ and $z=0$. In region $A^{\prime \prime}$ the circulation is confined between $z=-H$ and $z=-D^{\prime}$. In region $B$ the water moves top to bottom and topography is felt. In region $\mathrm{C}$ the fluid is at rest on $z=-D$ and $z=-H$.

b) Horizontal section at $z=-H$. The boundary between regions $\mathrm{B}$ and $A^{\prime \prime}$ is the projection of the folding curve resulting from the intersection of regions $A^{\prime}$ and $A^{\prime \prime}$ shown in Fig. 11a. 
The projection of this curve on the $x-y$ plane is shown in Fig. $2.11 \mathrm{~b}$ as the boundary between regions $A^{\prime \prime}$ and $\mathrm{B}$.

Inside region $B$ the fluid moves top to bottom and the circulation is given by

$$
F H^{2} \psi_{z z}+\beta y=\beta y_{1}
$$

with boundary conditions $\quad J\left(\psi, F H \psi_{z}\right)=-f_{0} w_{e} / H \quad$ at $\quad z=0$

$$
\text { and } F H \psi_{z}+f_{0} h / H=Q_{0} \quad \text { at } z=-H
$$

In this region, the vertically integrated transport, defined as

$$
\bar{\psi}=H^{-1} \int_{-H}^{0} d z \psi
$$

satisfies the equation

$$
J\left(\bar{\psi}, \beta y+f_{0} h / H\right)=f_{0} w_{e} / H+f_{0} h \beta /(6 F H)
$$

and its characteristics are the same as in the layer model, Eq. 2.2.6. The characteristics (2.2.6) intersect the boundary of region $B$ twice in the interior, leading to a mismatch of the flow occuring at all depths. Explicit solutions were not obtained given the complexity due to the presence of so many different regions.

In summary it appears that the occurrence of a mismatch in an interior region of the basin is a recurring property of baroclinic gyres, in the presence of $x$-dependent topography, regardless of the vertical resolution. 


\subsection{Ridge-like topography}

In this section we analyze the effects of a bottom topography of varying slope on both a barotropic and a two layer model on a $\beta$-plane. Topography will be ridge-like, i.e.

$$
h= \begin{cases}-h_{x}\left|x-x_{0}\right|+h_{x} a & \text { for }\left|x-x_{0}\right|<a \\ 0 & \text { for }\left|x-x_{0}\right|>a\end{cases}
$$

where $x_{0}$ is the point of maximum height $h_{x} a$, and $a$ is the halfwidth of the ridge. Let's first look at the case of one layer forced by a wind stress curl which is always negative, for example that of Eq. 2.2.4 with $y$ between 0 and $2 y_{0}$ (the one layer problem was solved by J. Pedlosky). The equation governing the flow will be

$$
J\left(\psi_{1}, \beta y+f_{0} h / H\right)=f_{0} w_{e}(y) / H
$$

The characteristics of the flow will be the geostrophic contours $\xi=\beta y+f_{0} h / H$, which are piecewise continuous broken lines dividing the domain in 8 different regions (see Fig. 2.12). If we require the flow to be zero at the eastern boundary and at the zeroes of the Ekman pumping we obtain the following results for the streamfunction for the different regions shown in Fig. 2.12.

$$
\begin{aligned}
\psi_{1}= & f_{0} w_{e}(y)\left(x-x_{e}\right) /(\beta H) \\
\psi_{1}= & \frac{1}{h_{x}} \int_{\xi / \beta}^{y} d \eta w_{e}(\eta)+f_{0} w_{e}(\xi / \beta)\left(x_{0}+a-x_{e}\right) /(\beta H) \\
\psi_{1}= & \frac{1}{h_{x}} \int_{0}^{y} d \eta w_{e}(\eta) \\
\psi_{1}= & -\frac{1}{h_{x}} \int_{0}^{y} d \eta w_{e}(\eta) \\
\psi_{1}= & -\frac{1}{h_{x}} \int_{s(\xi)}^{y} d \eta w_{e}(\eta)+\frac{1}{h_{x}} \int_{2 y_{0}}^{s(\xi)} d \eta w_{e}(\eta) \\
\psi_{1}= & -\frac{1}{h_{x}} \int_{s(\xi)}^{y} d \eta w_{e}(\eta)+\frac{1}{h_{x}} \int_{2 y_{0}}^{s(\xi)} d \eta w_{e}(\eta) \\
& +f_{0} w_{e}(\xi / \beta)\left(x_{0}-a-x_{e}\right) /(\beta H) \\
\psi_{1}= & -\frac{1}{h_{x}} \int_{0}^{y} d \eta w_{e}(\eta)+f_{0} w_{e}(y)\left(x+a-x_{e}\right) /(\beta H) \\
\psi_{1}= & -\frac{2}{h_{x}} \int_{s(\beta y)}^{y} d \eta w_{e}(\eta)+f_{0} w_{e}(y)\left(x+2 a-x_{e}\right) /(\beta H)
\end{aligned}
$$


where $s(\xi)=\xi / \beta-f_{0} h_{x} a /(\beta H)$.

Notice that the flow in regions $2 \mathrm{~A}$ and $3 \mathrm{C}$ is perfectly zonal and a discontinuity arises at the boundary between regions $\mathrm{C}$ and $\mathrm{B}$, in addition to discontinuities at the western and parts of the northern and southern boundaries. The difference in transport between regions $\mathrm{B}$ and $\mathrm{C}$ is given by

$$
\begin{aligned}
T_{\mathrm{B}-\mathrm{C}} & =H\left[\psi_{1}(3 \mathrm{~B})-\psi_{1}(3 \mathrm{C})\right]_{\xi=f_{0} h_{x} a /(\beta H)} \\
& =f_{0}^{2} W h_{x} a\left(x_{0}+a / 2-x_{e}\right) /\left(\beta^{2} y_{0} H\right)
\end{aligned}
$$

and is constant.

The physical mechanism of this discontinuity can be understood by means of the following analogy. Equation 2.5.1 is equivalent to the equation for the concentration of a passive tracer advected by a known velocity field ( here the concentration is analogous to $\psi_{1}$ and the known velocity field has flow lines identical to the geostrophic contours $\xi)$.

In the absence of forcing and dissipation the concentration will be constant along the flow lines and will be completely determined by the knowledge of the concentration at the boundaries of the domain under consideration. On the other hand, there might be some regions in the interior of the domain (such as the line dividing regions $\mathrm{C}$ and $B$ in Fig. 2.12) where neighboring flow lines trace back to regions of the boundaries that are very far apart and thus might have rather different concentration values. Therefore although the concentration is continuous along the boundary it may become discontinuous in the interior.

This explains also why in our oceanic, wind-forced problem the jump in transport across the discontinuity is constant. In the presence of forcing, the flow is the sum of two components: one across and the other along the geostrophic contours. The former is determined by the forcing and is continuous on the characteristic dividing regions $\mathrm{C}$ and $\mathrm{B}$ as long as the forcing is continuous there. The discontinuity arises in 


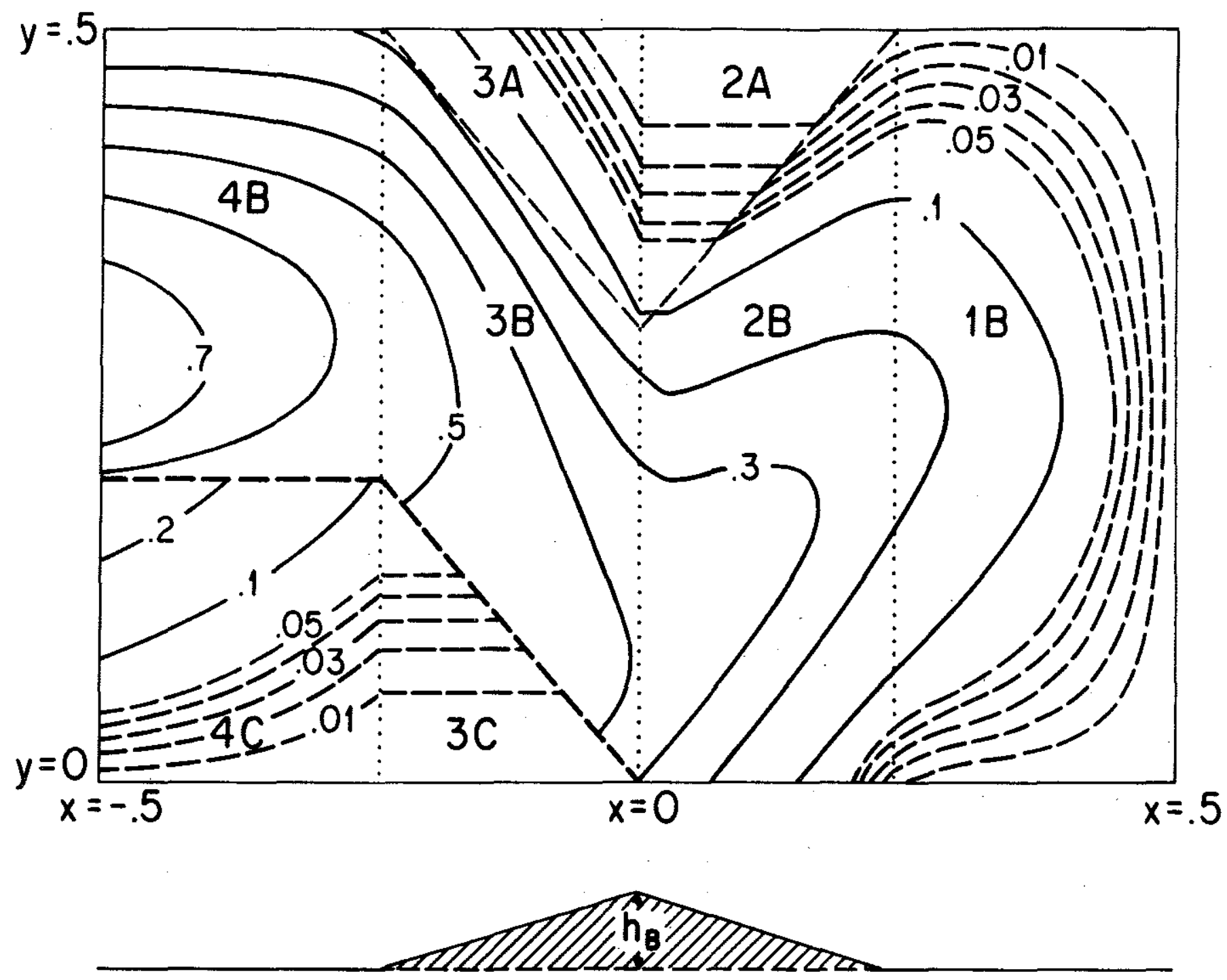

Fig. 2.12. Streamlines for the flow of the barotropic model in the presence of ridge-like topography. The thin dashed line represents one of the characteristics dividing the domain into different regions. The thick dashed line represents the characteristic at the location of the discontinuity. 
the component along the characteristics (the only one in the passive tracer analogue) which is obviously constant on a characteristic.

To emphasize the dependence upon the parameters we notice that the transport across the discontinuity can be written as

$$
T_{\mathrm{B}-\mathrm{C}}=T_{\mathrm{s}} \frac{\Delta h / H}{\Delta f / f_{0}} \frac{\left(x_{0}+a / 2-x_{e}\right)}{x_{e}}
$$

where $T_{s}=f_{0} W x_{e} / \beta$ is the maximum Sverdrup transport in the absence of topography, $\Delta h / H=h_{x} a / H$ is the relative change in height due to the topography and $\Delta f / f_{0}=$ $\beta y_{0} / f_{0}$ is the characteristic change in Coriolis parameter. For $x_{e}=6000 \mathrm{~km}, f_{0}=$ $10^{-4} \mathrm{sec}^{-1}, W=10^{-4} \mathrm{~cm} \mathrm{sec}^{-1}, y_{0}=1000 \mathrm{~km}, H=4000 \mathrm{~m}, \beta=10^{-13} \mathrm{~cm}^{-1} \mathrm{sec}^{-1}, \Delta h=$ $1000 \mathrm{~m}, x_{0}=3000 \mathrm{~km}, a=1200 \mathrm{~km}, T_{s}=60 \mathrm{~Sv}$ we get $T_{\mathrm{B}-\mathrm{C}}=60 \mathrm{~Sv}$. This value is clearly an upper limit for the transport at the jump since the barotropic model velocities are very large at the bottom. Also the ridge height and the basin width are very large in this example, at the limit of applicability of the quasi-geostrophic approximation. Nevertheless this value is indicative of the fact that these jets have transport of the same order as the interior flow.

We now want to see what are the effects of this ridge-like topography on the two layer model, considered in section 2.2. Again the forcing will be that of Fig. 2.1. We will take the streamfunction in the lower layer such as to keep $q_{2}$ constant inside the outermost closed $\hat{q}_{2}$ contour and $\psi_{2}$ zero outside where now

$$
\hat{q}_{2}=\beta y+\frac{f_{0} h_{x}}{H_{2}}\left(-\left|x-x_{0}\right|+a\right)+\left(x-x_{e}\right) \frac{f_{0} w_{e}(y) F_{2}}{\beta H_{1}}
$$

An example is given in Fig. 2.13. The characteristics of the flow will be

$$
\xi=\beta y+f_{0} h /\left(H_{1}+H_{2}\right)
$$

and they divide the region of lower layer flow into several parts shown in Fig. 2.13. Similarly to the barotropic case, in the lower layer there are two regions of weak quasizonal flow (2B and 3D in Fig. 2.13) and a discontinuity in flow between regions $\mathrm{C}$ and 
D. The jump in the streamfunction is the same in the two layers because of the choice of constant potential vorticity. The vertically integrated difference in transport at the jump is given by

$$
T_{\mathrm{C}-\mathrm{D}}=\frac{f_{0} h_{x} a}{\beta\left(H_{1}+H_{2}\right)}\left[\beta H_{1} / F 2+f_{0} W\left(x_{0}+a / 2-x_{e}\right) /\left(\beta y_{0}\right)\right]
$$

and is always larger than the corresponding value for the one layer model. A mismatch analogous to that of section 2.2 occurs at the northern boundary of region $3 \mathrm{~B}$ inducing a discontinuity which continues with constant value at the boundary between regions 4A and 4B. In Table 2.2 some values for this jump in the lower layer streamfunction are given for different values of the topographic slope, the width of the ridge and the strength of the forcing. The nondimensional slope $f_{0} h_{x} /(\beta H)=2$ and the nondimensional halfwidth $a / x_{e}=0.2$ in Table 2.2 a correspond to the dimensional values given in the previous example for the homogeneous model. This gives a dimensional total transport of $60 \mathrm{~Sv}$, which fortuitously happens to be the same value as the internal jet transport for the barotropic model, and again this value is comparable to the interior transport. Notice that this discontinuity grows more slowly with the topographic slope than $T_{\mathrm{C}-\mathrm{D}}$, while doubling the width of the ridge more than doubles the amplitude of the discontinuity. This is what one would actually expect since the "internal" jump (C - D) owes its existence to the change in slope of the bottom topography. In fact taking a very thin ridge, but with constant height $\left(h_{x} a=\right.$ constant, $\left.a \rightarrow 0\right)$ the internal jump would continue to exist with almost unchanged strength while the mismatch at the northern boundary of region $3 \mathrm{~B}$ tends to vanish. For reasonable values of the parameters both these discontinuities are of the same magnitude as the interior flow and they arise in regions away from (although connected to) the solid boundaries of the domain. 


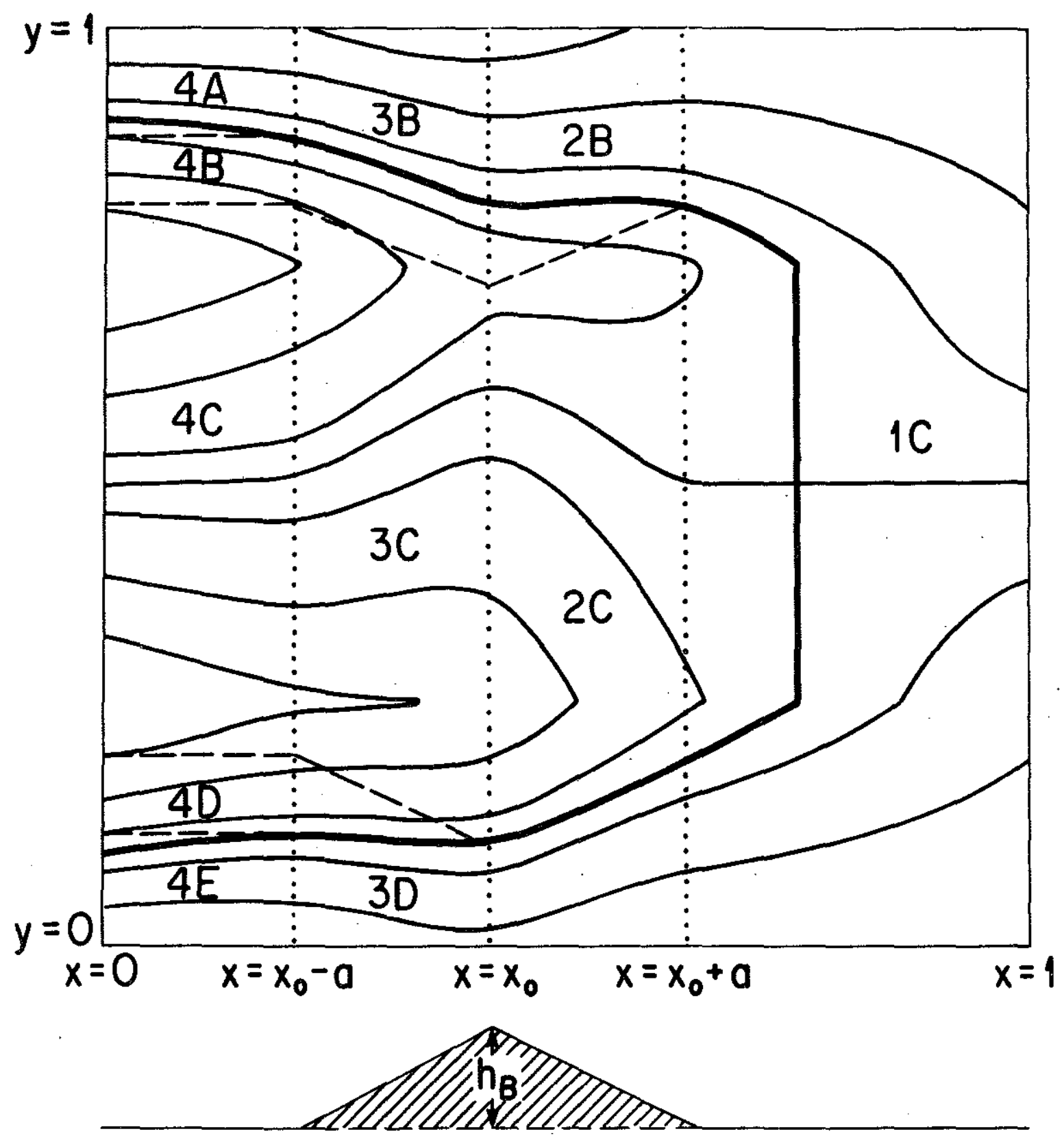

Fig. 2.13. Contours of $\hat{q}_{2}$. The heavy line represents the outermost closed contour and the dashed lines are the characteristics dividing the domain in different regions. $f_{0} W F_{2} /\left(\beta^{2} H_{1}\right)=-1 ., f_{0} h_{x} /\left(\beta H_{2}\right)=0.8, x_{0} / x_{e}=0.4, a / x_{e}=0.2$. 


\begin{tabular}{|c|c|c|c|c|c|}
\hline \multirow{2}{*}{ Ridge halfwidth } & \multicolumn{5}{|c|}{ Topographic slope } \\
\cline { 2 - 6 } & 0.4 & 0.8 & 1.2 & 1.6 & 2.0 \\
\hline 0.1 & -0.04 & -0.07 & -0.09 & -0.10 & -0.11 \\
0.2 & -0.09 & -0.14 & -0.18 & -0.22 & -0.25 \\
0.3 & -0.14 & -0.22 & -0.28 & -0.34 & -0.37 \\
\hline
\end{tabular}

\begin{tabular}{|c|c|c|c|c|c|}
\hline \multirow{2}{*}{ Ridge halfwidth } & \multicolumn{5}{|c|}{ Topographic slope } \\
\cline { 2 - 6 } & 0.4 & 0.8 & 1.2 & 1.6 & 2.0 \\
\hline 0.1 & -0.06 & -0.08 & -0.10 & -0.11 & -0.12 \\
0.2 & -0.12 & -0.18 & -0.22 & -0.27 & -0.30 \\
0.3 & -0.18 & -0.29 & -0.39 & -0.45 & -0.47 \\
0.4 & -0.26 & -0.43 & -0.56 & -0.58 & -0.56 \\
\hline
\end{tabular}

Table 2.2: Difference in transport, for the ridge-like topography, in the lower layer at the northern jump (region 4A-4B) for different values of the topographic slope $f_{0} h_{x} /\left(\beta H_{2}\right)$ (columns), and the ridge halfwidth $a / x_{e}$ (rows). Top) The nondimensional forcing is $f_{0} W F_{1} /\left(\beta^{2} H_{1}\right)=-1.0$. Bottom) The nondimensional forcing is $f_{0} W F_{1} /\left(\beta^{2} H_{1}\right)=$ -2.0 .

\subsection{Discussion}

We have shown how in the presence of topography internal jets arise when simple Sverdrup dynamics are used. More specifically we find that for baroclinic gyres, jets are formed at the boundary of the region containing the subsurface flow. Their existence is independent of the forcing mechanism for the lower layer as long as such forcing is small. On the other hand their very presence raises questions about the applicability of conservative dynamics since at the jets' locations, frictional effects have to be much stronger than we considered initially. To avoid such difficulties De Szoeke (1985), in an independent work on the same subject, has chosen to prescribe the flow as completely continuous in both layers. This choice implies that either the forcing has to be of a special form or that the eastern boundary condition can't be applied in the upper layer. 
We think, however, that it is inescapable to embed the circulation in a closed basin and the occurrence of flows with internal jets must not be ruled out a priori.

Since our results are not sensitive to the choice of constant potential vorticity in the lower layer we need not to restrict ourselves to constant slope topography. Therefore we have also analyzed the effects of bottom topography of varying slope, in particular in the form of a ridge. Again jets are found although of a different nature. In fact in this case, internal jets occur in both stratified and even in homogeneous models; their existence now is due to the change in sign of the bottom slope and the presence of the southern gyre boundary along which $\psi$ vanishes. For values of the parameters in the oceanic range, the jets produced both in the ridge-like and in the constant slope topography have very large transports, i.e., on the order of the maximum transport of the interior flow.

In the next chapter we will examine numerical models that include relative vorticity and small nonconservative mechanisms to see whether the features found in the previous sections actually occur, and how they are modified by higher order effects. 


\section{Appendix A2. Viscous boundary layers analysis}

Assuming that lower layer potential vorticity is constant in the boundary layer, the equation for the barotropic flow in the presence of small bottom friction is:

$$
\left(1+H_{2} / H_{1}\right) \psi_{2 x}-\frac{f_{0} h_{x}}{\beta H_{1}} \psi_{2 y}=\frac{f_{0} w_{e} F_{1}}{\beta^{2} H_{1}}+\frac{f_{0} h_{x}}{\beta H_{1}}-\frac{\delta H_{2}}{\beta L H_{1}} \nabla^{2} \psi_{2}
$$

The boundary of validity of constant potential vorticity dynamics is

$$
Q_{2} / \beta=y+\frac{f_{0} h_{x}}{\beta H_{2}}\left(x-x_{e}\right)+\frac{f_{0} W F_{2} f(y)}{\beta^{2} H_{1}}\left(x-x_{e}\right)
$$

Therefore, we define as boundary layer variable

$$
\epsilon=y+\left(x-x_{e}\right)\left(r+w_{0} f(y)\right)-Q_{2} / \beta
$$

where

$$
r=f_{0} h_{x} /\left(\beta H_{2}\right), \quad w_{0}=f_{0}^{3} W /\left(\beta^{2} g^{\prime} H_{1} H_{2}\right)
$$

and

$$
\lambda=x-x_{e} \text { as the other independent variable. }
$$

The dominant balance is, for $\epsilon=\delta H_{2} /\left(\beta L H_{1}\right)$ :

$$
c(\lambda) \frac{\partial \psi_{2}}{\partial \xi}+g(\lambda) \frac{\partial^{2} \psi_{2}}{\partial \xi^{2}}=0
$$

where $g(\lambda)$ is always positive:

and

$$
\begin{aligned}
& g(\lambda)=\left[\frac{r+4 w_{0}\left(Q_{2} / \beta-1\right)}{1+4 w_{0} \lambda}\right]^{2}+\left(1+4 w_{0} \lambda\right)^{2} \\
& c(\lambda)=\frac{2\left[r+4 w_{0}\left(Q_{2} / \beta-1\right)\right]-r\left(1+4 w_{0}\right)^{2}}{1+4 w_{0} \lambda}
\end{aligned}
$$

In order to get solutions which are decaying for $\xi \rightarrow-\infty$ (inside the region of constant potential vorticity) we must have $c(\lambda)<0$, which gives:

$$
\lambda<\frac{1-\sqrt{2+8 w_{0}\left(Q_{2} / \beta-1\right) / r}}{4\left|w_{0}\right|}
$$


This is exactly the relation which determines the point at which the boundary is tangent to the characteristics. For $x-x_{e}$ greater than this value the boundary layer streamfunction grows exponentially but the amplitude of the boundary layer correction goes to zero.

$$
\psi_{2}(\xi, \lambda)=A(\lambda)[1-\exp (-g(\lambda) \xi / c(\lambda))]
$$

where

$$
A(\lambda)=-2 w_{0}\left(Y-Y_{s}\right)\left(Y+Y_{s}-2+r /\left(2 w_{0}\right)\right) / r
$$

with

$$
Y=\left(Q_{2} / \beta-r \lambda+4 w_{0}\right) /\left(1+4 w_{0} \lambda\right)
$$

and

$$
Y_{s}=r \dot{\lambda} / 2+1-r /\left(8 w_{0}\right)
$$

At the point given by (A2.1) $Y=Y_{s}$ and $A(\lambda)=0$.

If the Ekman pumping is of a more general form (see Fig. A2) (here the variables are nondimensional):

$$
w(y)= \begin{cases}w_{m} y / y_{1} & \text { if } y<y_{1} \\ {\left[\left(w_{M}-w_{m}\right) y+y_{2} w_{m}-y_{1} w_{M}\right] /\left(y_{2}-y_{1}\right)} & \text { if } y_{1} \leq y \leq y_{2} \\ w_{M}(y-1) /\left(y_{2}-1\right) & \text { if } y_{2} \leq y \leq y_{1}\end{cases}
$$

The point at which the outermost closed $q_{2}$ contour is tangent to the characteristics is:

$$
\lambda=\left(y_{2}-1\right)(2 b-1) / w_{M}
$$

where

$$
b=\frac{\left(1+w_{M} / r\right)\left[w_{M}\left(1-y_{1}\right)-w_{m}\left(1-y_{2}\right)\right]}{2\left(1-y_{2}\right)\left(w_{M}-w_{m}\right)}
$$




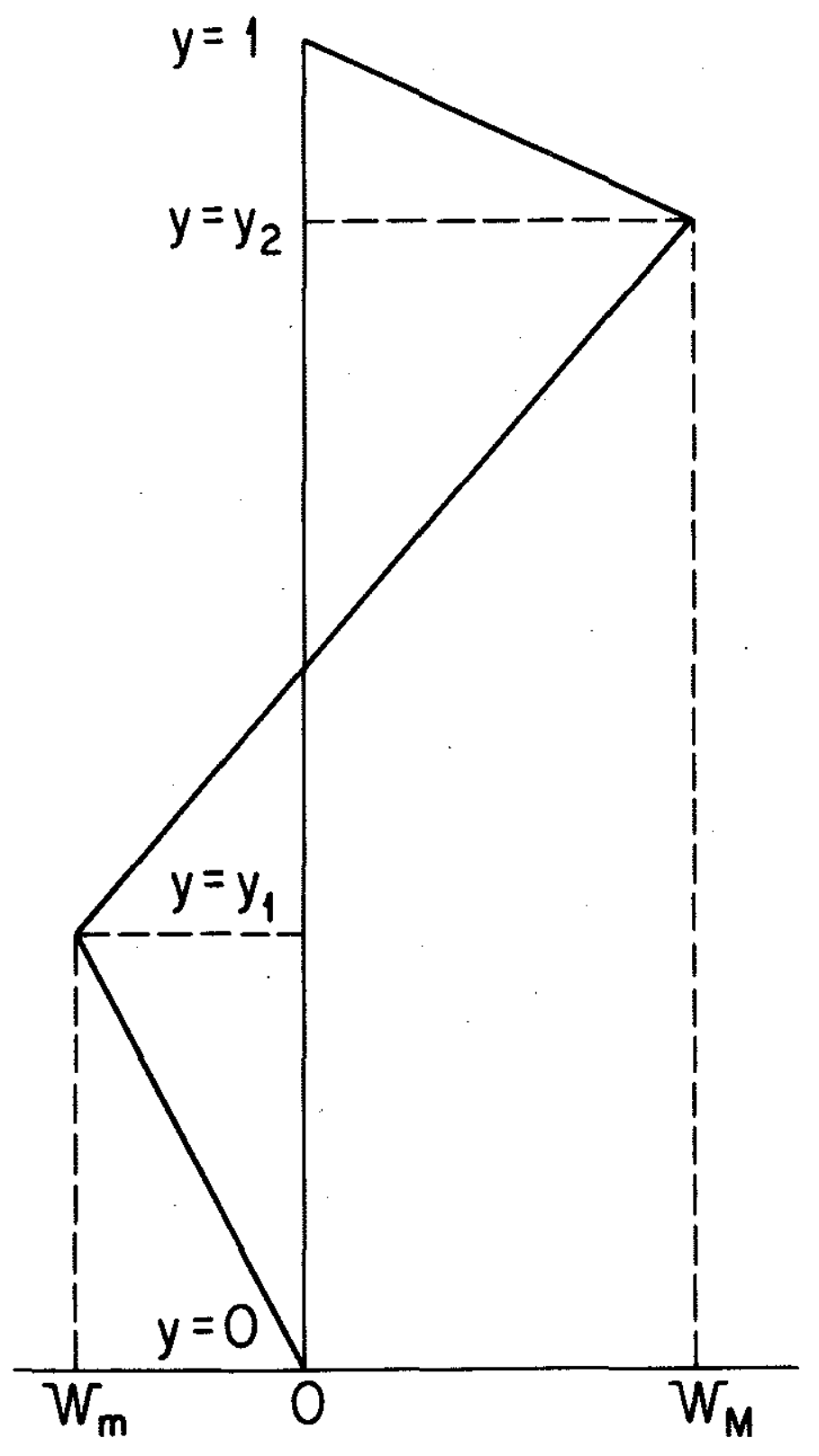

Fig. A2. The Ekman pumping (A2.2) as a function of $y$. 


\section{Appendix B2. Homogeneous model in the presence of topography}

The equation governing the transport of one layer of homogeneous fluid in the presence of topography $h=\alpha x$, forced by a longitude independent Ekman pumping is:

$$
\beta \psi_{b x}-\frac{f_{0} \alpha}{H} \psi_{b y}=\frac{f_{0} w_{e}(y)}{H}
$$

Setting $\xi=y+f_{0} \alpha\left(x-x_{e}\right) /(\beta H)=y+r\left(x-x_{e}\right)$ and choosing $r>0$, its solution is:

$$
\psi_{b}=-\int^{y} d \eta w_{e} / \alpha+\phi(\xi) .
$$

Given the form of the modified $\beta$-effect we choose to satisfy the following boundary conditions:

$$
\psi_{b}=0 \quad \text { on } \quad x=x_{e} \quad \text { and } \quad \psi_{b}=0 \text { on } y=0 .
$$

Therefore the flow is divided into two regimes:

$$
\psi_{b}=-\int_{y+r\left(x-x_{e}\right)}^{y} d \eta w_{e} / \alpha \quad \text { for } \xi>0
$$

and

$$
\psi_{b}=-\int_{0}^{y} d \eta w_{e} / \alpha \quad \text { for } \xi<0
$$

Because of the choice of an $x$-independent forcing, in the south-west part of the basin the flow is perfectly zonal. Notice also how the cyclonic gyre is reduced in favour of the subtropical gyre (see Fig. B2). 


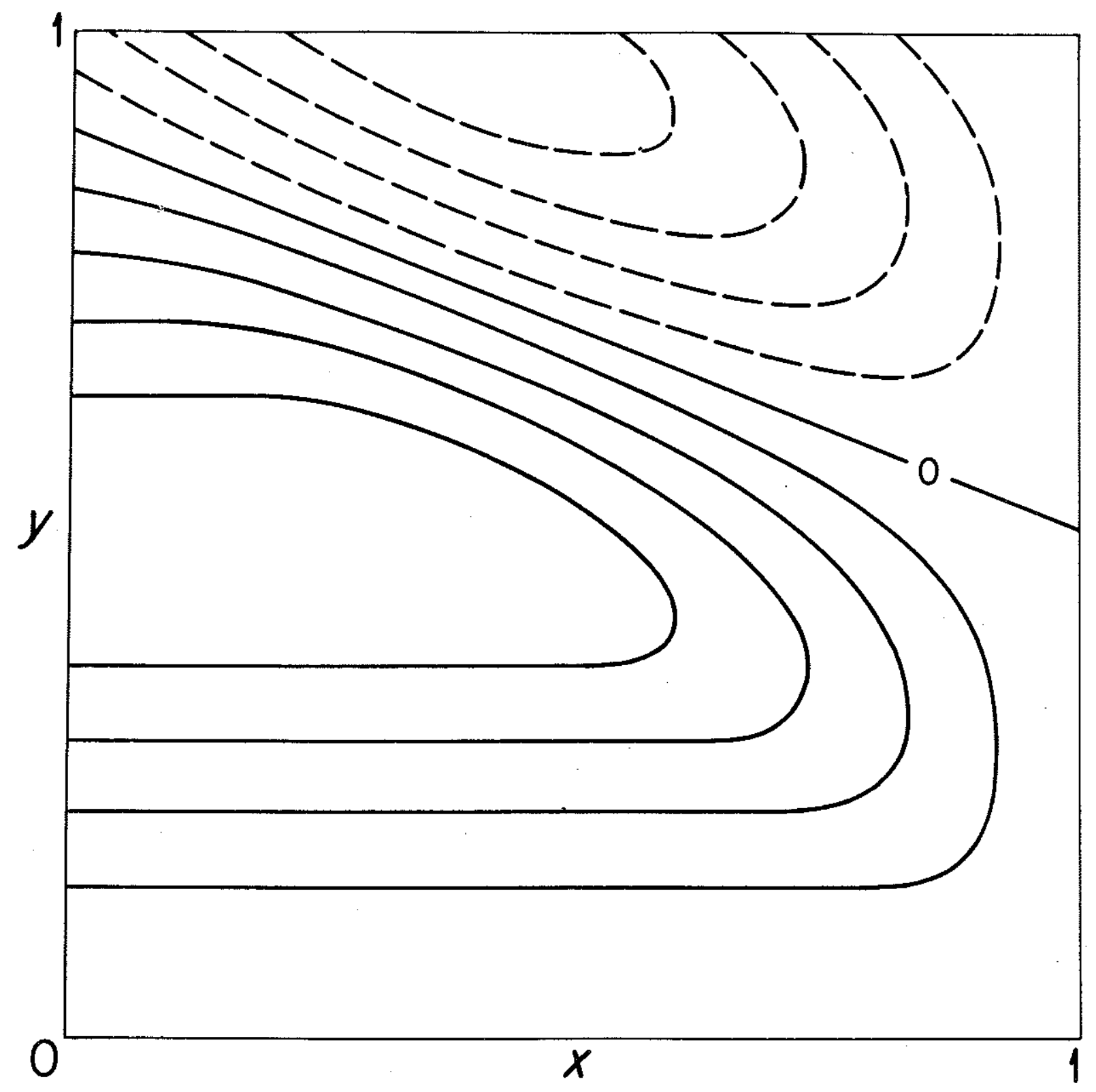

Fig. B2: Streamlines for the barotropic streamfunction resulting from the Ekman pumping $w_{e}=W \sin (2 \pi y)$. With $f_{0} W /\left(\beta^{2} g H^{2}\right)=-1.2$ and $f_{0} \alpha /(\beta H)=0.8$. 
Appendix C2. The constant value of potential vorticity within the "bowl". For a continuosly stratified model, the homogenization argument of Rhines and Young (1982a) leads to the result that, within the "bowl" containing the motion, potential vorticity is independent of $x$ and $y: q=\bar{q}(z)$. Since $\bar{q}(z)$ is independent of the horizontal coordinates, its value is more easily found in the region $D<H$ where the bowl containing the circulation does not hit the bottom and topography is not felt.

The calculation will be performed under the assumption that the Brunt-Vaisala frequency $N^{2}$ is constant, but the result holds for an arbitrary stratification. In the region $D<H$ the equation governing the flow is

$$
F H^{2} \psi_{z z}+\beta y=\bar{q}(z)
$$

with boundary conditions $J\left(\psi, F H \psi_{z}\right)=-f_{0} w_{e} / H$ at $z=0$ and $\psi=\psi_{z}=0$ at $z=$ $-D$. It is clear that in the region $D<H$ there will be no flow across the zero wind stress curl line, $y=2 y_{0}$, therefore $\psi=\psi_{z}=\psi_{z z}=0$ at that latitude, and thus the value of $\bar{q}(z)$ can be calculated at $y=2 y_{0}$ from (C2.1), so $\bar{q}(z)=2 \beta y_{0}$, which is a constant independent of $z$. The choice $\psi=\psi_{z}=\psi_{z z}=0$ at the eastern wall, and thus at the zero Ekman pumping latitude, can always be made by defining the mean stratification $N^{2}$ appropriately.

The result that the latitude of zero Ekman pumping is a streamline in the region $D<H$ can be proved without knowledge of the value of $\bar{q}(z)$. Integrating (C2.1) vertically from $z=-D$ to an arbitrary depth $z \leq 0$ and using the boundary conditions at $z=-D$ we find

$$
\begin{aligned}
F H^{2} \psi_{z} & =-(z+D) \beta y+\int_{-D}^{z} d z^{\prime} \bar{q}\left(z^{\prime}\right) \\
F H^{2} \psi & =-(z+D)^{2} \beta y / 2+\int_{-D}^{z} d z^{\prime} \int_{-D}^{z^{\prime}} d z^{\prime \prime} \bar{q}\left(z^{\prime \prime}\right)
\end{aligned}
$$

Application of the boundary condition at $z=0$ gives

$$
[\bar{q}(-D)-\beta y] \beta D^{2} D_{x} / 2=f_{0} w_{e} F H^{2}
$$


On the other hand the meridional velocity is given, from (C2.2), by

$$
F H^{2} \psi_{x}=-(z+D) D_{x}[\beta y-\bar{q}(-D)]
$$

Using (C2.3) to eliminate $D_{x}[\beta y-\bar{q}(-D)]$ from (C2.4) one finds

$$
\psi_{x}=f_{0} w_{e}(z+D) /\left(\beta D^{2}\right)
$$

If $y=2 y_{0}$ is the zero Ekman pumping latitude then $\psi\left(x, 2 y_{0}\right)=0$ for every $x$ and $z$, and as expected, there is no meridional flow across the latitude of zero Ekman pumping. 


\section{CHAPTER 3}

On the role of topography

in the wind-driven circulation:

viscous and inertial effects 


\subsection{Numerical experiments}

In the previous chapter we have shown that, in the presence of $x$-dependent topography, internal jets arise when Sverdrup dynamics are used. It is not obvious a priori that all the assumptions made about the higher order effects will hold when, for example, viscosity is explicitly introduced in the calculations. It is also conceivable that, when relative vorticity is restored, the internal jets will become unstable, violating the assumption that the solutions found in the previous chapter represent possible steady states. Thus both relative vorticity and dissipative terms will be explicitly included in the calculations presented in this chapter.

A special comment should be made about the role of relative vorticity. Ierley and Young (1983) have shown that, in the absence of relative vorticity, the western boundary layer dynamics affect the interior flow when interfacial friction and bottom drag are taken into account in quasigeostrophic, wind-driven layer models. In their calculation, because all the flow lines go through a frictional western boundary current, the homogenization arguments of Rhines and Young (1982) do not apply and the relation between the streamfunction and the potential vorticity in the interior of the unforced layers is not simple.

In the analysis presented in Ierley and Young (1983), the western boundary viscous dynamics play an essential role in the "exit" region, located at the northwest corner in the subtropical gyre. In models which include relative vorticity, such as that of Holland et al. (1984), a recirculating gyre is established at the "exit" region, and many of the interior streamlines pass through the northern boundary layer sandwiched between the recirculating gyre and the zero wind stress curl line, invalidating the arguments presented in Ierley and Young (1983). For reasons that are yet unclear, the occurrence of the recirculating gyre favors homogenization of potential vorticity in the unforced layers. 
Because all the calculations in the previous chapter have been done under the assumption that potential vorticity is constant in the subsurface layers it would be desirable to be able to compare the inviscid results with those obtained using finite viscosity. Thus both relative vorticity and diffusion will be taken into explicit account in the following calculations.

The inclusion of relative vorticity and of weak, but finite diffusion makes the problem analyzed in the previous sections analytically untractable. Solutions were found by means of a numerical model developed by Dr. Ierley. A brief description of the model is given in Appendix A3. The numerical model solves the quasigeostrophic equations

$$
\begin{array}{ll}
\frac{\partial q_{1}}{\partial t}+J\left(\psi_{1}, q_{1}\right)=f_{0} w_{e} / H_{1}+\kappa \nabla^{2} q_{1} \\
\frac{\partial q_{2}}{\partial t}+J\left(\psi_{2}, q_{2}\right)=-\delta \nabla^{2} \psi_{2}+\kappa \nabla^{2} q_{2} \\
\text { where } \quad \begin{aligned}
q_{1} & =\nabla^{2} \psi_{1}+F_{1}^{*}\left(\psi_{2}-\psi_{1}\right)+\beta y \\
q_{2} & =\nabla^{2} \psi_{2}+F_{2}^{*}\left(\psi_{1}-\psi_{2}\right)+\beta y+f_{0} h_{b} b(x, y) / H_{2}
\end{aligned}
\end{array}
$$

with boundary conditions $\psi_{1}=\psi_{2}=\nabla^{2} \psi_{1}=\nabla^{2} \psi_{2}=0$ on $x= \pm L_{x}, y= \pm L_{y}$. $w_{e}=w g(x, y)$ is the Ekman pumping applied at the top of the upper layer, $\kappa$ is the coefficient of lateral diffusion of potential vorticity, $\delta$ is the coefficient of bottom friction, $F_{1}^{*}=f_{0}^{2} /\left(g^{\prime} H_{1}\right)$ is the inverse of the squared Rossby radius of deformation, $F_{2}^{*}=F_{1}^{*} H_{1} / H_{2}$, and $h_{b}$ is a typical height of the bottom elevation while $b(x, y)$ is the form of the topography. There are thus six dimensional external parameters which determine the solution. Numerical solutions are more conveniently found when (3.1.1) 
is put in nondimensional form with the choice

$$
\begin{aligned}
(x, y) & =L_{y}\left(x^{\prime} / \epsilon, y^{\prime}\right) \quad \epsilon=L_{y} / L_{x} \\
\psi_{n} & =\frac{L_{y} f_{0} w}{\epsilon \beta H_{1}} \psi_{n}^{\prime} \\
t & =\frac{\beta L_{y} H_{1}}{f_{0} w} t^{\prime} \\
q_{n} & =\beta L_{y} q_{n}^{\prime} \\
\kappa & =\frac{f_{0} w L_{y}}{\beta H_{1}} \kappa^{\prime} \\
\delta & =\epsilon \beta L_{y} \delta^{\prime}
\end{aligned}
$$

Dropping the primes (3.1.1) becomes

$$
\begin{aligned}
& \frac{\partial q_{1}}{\partial t}+J\left(\psi_{1}, q_{1}\right)=g(x, y)+\kappa \nabla^{2} q_{1} \\
& \frac{\partial q_{2}}{\partial t}+J\left(\psi_{2}, q_{2}\right)=-\delta \nabla^{2} \psi_{2}+\kappa \nabla^{2} q_{2} \\
& \text { where } \quad q_{1}=\gamma \nabla^{2} \psi_{1}+F_{1}\left(\psi_{2}-\psi_{1}\right)+y \\
& q_{2}=\gamma \nabla^{2} \psi_{2}+F_{2}\left(\psi_{1}-\psi_{2}\right)+y+h b(x, y) \\
& \text { and } \quad \nabla^{2}=\frac{\partial^{2}}{\partial y^{2}}+\epsilon^{2} \frac{\partial^{2}}{\partial x^{2}}
\end{aligned}
$$

with boundary conditions $\psi_{1}=\psi_{2}=\nabla^{2} \psi_{1}=\nabla^{2} \psi_{2}=0$ on $x= \pm 1, y= \pm 1$. All the quantities are now nondimensional. If a velocity scale is defined as $U=f_{0} w /\left(\epsilon \beta H_{1}\right)$ then $\gamma=U /\left(\beta L_{y}^{2}\right)$ is the square of the ratio of the inertial boundary layer thickness to the North-South basin scale, $F_{n}=F_{n}^{*} \epsilon U / \beta$ is the square of the ratio of the inertial scale to the Rossby deformation radius. $h=f_{0} h_{b} /\left(\beta L_{y} H_{2}\right)$ is the ratio of the topographic effect to the planetary vorticity gradient, $\kappa$ is now the inverse of the Reynolds number and $\delta$ is the ratio of the Stommel boundary layer width to the basin scale.

The purpose of this work is to study some processes rather than to simulate realistic oceanic features, therefore the values used for the parameters should reflect their order of magnitude only. With this in mind typical choices are $L_{y}=L_{x}=1000 \mathrm{~km}, g^{\prime}=.01$ $\mathrm{m} \mathrm{sec}{ }^{-2}, \beta=10^{-11} \mathrm{~m}^{-1} \mathrm{sec}^{-1}, f_{0}=10^{-4} \mathrm{sec}^{-1}, H_{1}=H_{2}=1000 \mathrm{~m}, \kappa_{d i m}=100 \mathrm{~m}^{2} / \mathrm{sec}$, 
$\delta_{\text {dim }}=10^{-7} \mathrm{sec}^{-1}, w=10^{-4} \mathrm{~cm} / \mathrm{sec}, h_{b}=100 \mathrm{~m}$. This gives, for the nondimensional parameters, the following order of magnitudes

$$
\begin{aligned}
\gamma & =10^{-3} \\
F_{n} & =1 \\
\kappa & =10^{-2} \\
\delta & =10^{-2} \\
h & =1
\end{aligned}
$$

For the values just quoted the nondimensional inertial boundary layer thickness $\gamma^{1 / 2} \approx 0.03$ while the Munk layer thickness is $(\kappa \gamma)^{1 / 3} \approx 0.02$. Thus the boundary layer thicknesses are of the same order and the western boundary dynamics are fully nonlinear. Three series of experiments were performed with different shapes of Ekman pumping $g(x, y)$ and bottom topography $b(x, y)$.

1) The Ekman pumping is of one sign (always negative as in the subtropical gyre) and is independent of longitude. The topography is in the form of a simple ridge independent of latitude.

2) The Ekman pumping is of one sign (the same as in the previous series), and the topography is in the form of a constant slope in the longitudinal direction.

3) The Ekman pumping is positive in the western half of the basin and negative in the eastern half, and depends on latitude as well. The topography is a constant slope in the longitudinal direction.

The purpose of these experiments is to observe the occurrence of the internal jets described in the previous chapter, in the presence of small diffusive processes and of relative vorticity. In the following I will describe the results obtained. 


\subsection{Ridge-like topography}

In the presence of a topography of varying slope, the main result of the previous chapter was the occurrence of an internal boundary layer connecting the western boundary layer to the topographically induced southern boundary layer (see Fig. 2.12). In the inviscid limit the internal boundary layer appeared as a flow discontinuity due to the change in sign of the bottom topography and it was found in both baroclinic and barotropic models.

In the two-layer model it was found that, in the absence of relative vorticity and of bottom friction, and with the assumption that $q=$ constant, the transport across the discontinuity was the same in both layers and therefore there was no jump in potential vorticity.

Three experiments will be discussed, all of them have an Ekman pumping of the form $g(x, y)=-\cos (\pi y / 2)$ and the bottom topography has the form $h b(x, y)=$ $A e^{-\left[\left(x-x_{0}\right) / a\right]^{2}}$. The values of the parameters used are summarized in Table 3.1.

\begin{tabular}{|c|c|c|c|c|c|c|c|}
\hline Experiment & $A$ & $a$ & $x_{0}$ & $\gamma$ & $\kappa$ & $\delta$ & $F_{1}=F_{2}$ \\
\hline R1 & 1.5 & 0.2 & -0.6 & 0.0005 & 0.018 & 0.009 & 4 \\
R2 & 1.5 & 0.2 & 0.0 & 0.0005 & 0.025 & 0.0125 & 6 \\
R3 & 1.5 & 0.2 & 0.0 & 0.0005 & 0.050 & 0.025 & 6 \\
\hline
\end{tabular}

Table 3.1: Summary of the parameters used for the experiments in the presence of a ridge-like topography of the form $h b=A e^{-\left[\left(x-x_{0}\right) / a\right]^{2}}$ and of an Ekman pumping of the form $g(x, y)=-\cos (\pi y / 2)$. All the quantities are nondimensional.

In the first experiment, $\mathbf{R} 1$, the ridge is centered in the western half of the basin $\left(x_{0}=-0.6\right)$, it has a halfwidth $a=0.2$ and its maximum height is $A=1.5$. The experiment was integrated in time until the steady state state was reached (see Appendix A2 for the definition of "steady state"). The prediction of the analytical calculations is that the lower layer should move only in the region of "closed contours" (the region where no contours are drawn in Fig. 3.1a) and should be at rest in the region of 
"blocked contours" (the region where the lower layer potential vorticity contours trace back to the southern, northern or eastern boundary). A strong jet should be observed along the characteristic marked by a thick line in Fig. 3.1b. In Figs. 3.2a,b the streamfunction fields are shown and indeed a crowding of the streamlines can be observed near the region of the dividing characteristic, although the flow intensification appears along a characteristic $\xi$ different than that indicated in Fig. 3.1b. The reason for this discrepancy is that there is some viscously driven lower layer flow outside the region of "closed contours". The formula obtained in the previous chapter for the vertically integrated difference in transport across the dividing characteristic is, in the present nondimensional units,

$$
T=A\left(F_{2}^{-1}-x_{0}-a^{\prime} / 2+1\right) / 2
$$

and is valid for the simple piecewise continuous topography and Ekman pumping used in Section 2.4. $A$ is the maximum height of the ridge and $a^{\prime}$ is the halfwidth of the piecewise continuous ridge. Although the form of the topography and of the Ekman pumping used in the numerical experiments are slightly different from those used in the analytical calculations, there is a qualitative similarity and I will nevertheless compare (3.2.3) to the numerical results.

Because the thickness of the internal boundary layer in the numerical experiment is finite, the choice of the streamfunction values on each side of the dividing characteristic is to some extent arbitrary. My choice from inspection of Fig. 3.2 is $\Delta \psi_{1}=0.80$ and $\Delta \psi_{2}=0.75$, so that the vertically integrated jump is $T=1.55$. For the values of the parameters used in this experiment (3.2.3) gives $T=1.31$, which is in good agreement with the numerical results. In Fig. 3.2c,d the potential vorticity fields are shown for the experiment R1. The lower layer potential vorticity gradients are greatly reduced in the region of closed contours, where the bulk of the abyssal flow occurs, with respect to the region of "blocked" contours. Notice that, in agreement with the theoretical prediction, there is no enhancement of the potential vorticity gradients in the region where the 

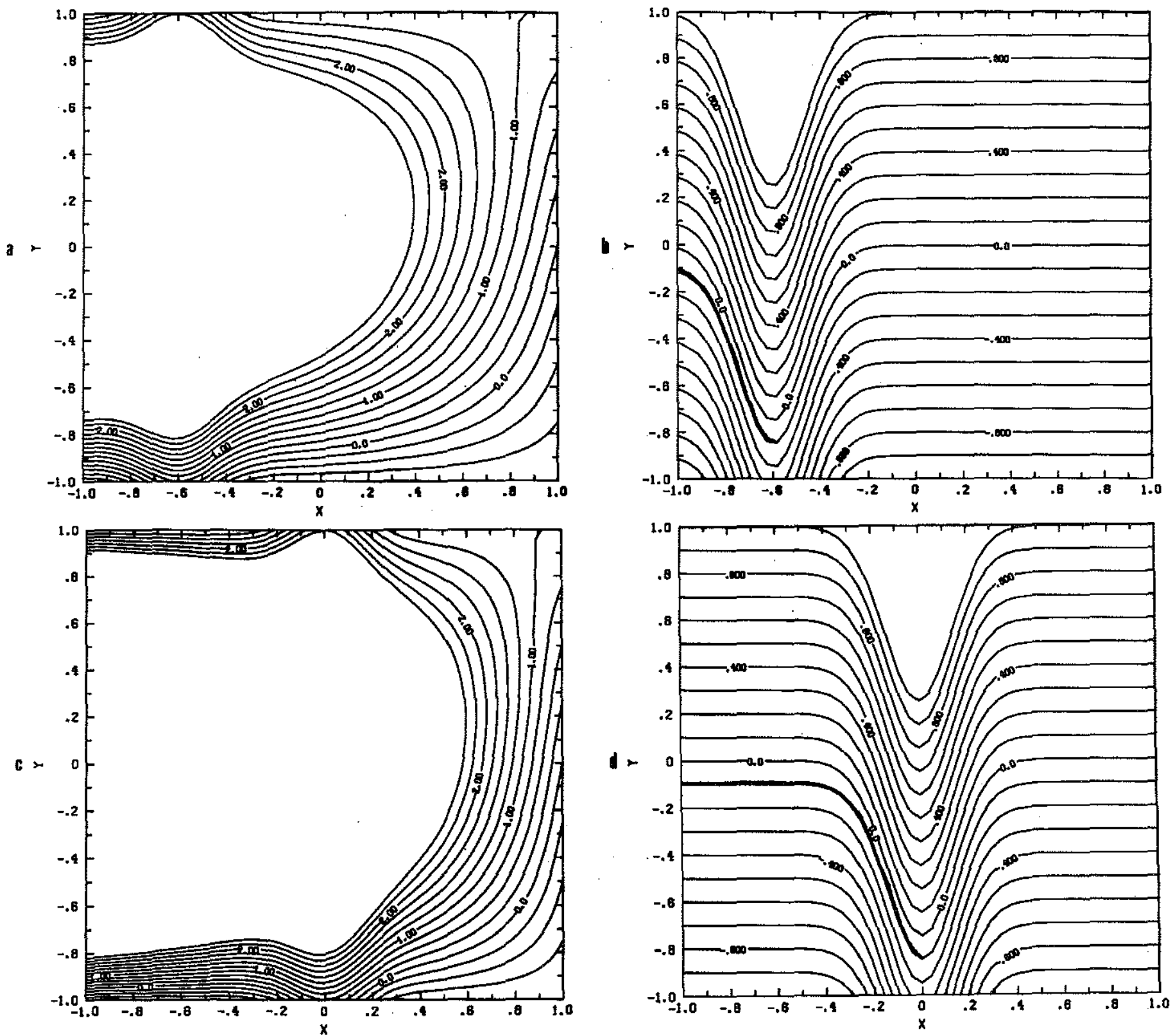

Fig. 3.1. a) Lower layer potential vorticity contours, as predicted by the inviscid calculation, for the parameters used in R1. The abyssal flow should be confined in the region where no contours are drawn ("closed contour"), and $q_{2}-\delta / \kappa \psi_{2}$ should be constant there. b) Contours of $\xi=y+h b / 2$ for the parameters used in R1. According to the inviscid calculation, in the "closed contours" region, the geostrophic contours for the upper layer flow are parallel to $\xi$. The internal jet should be observed along the thick line. c) Same as Fig. 3.1a, but for the parameters used in R2. d) Same as Fig. 3.1b, but for the parameters used in R2. 

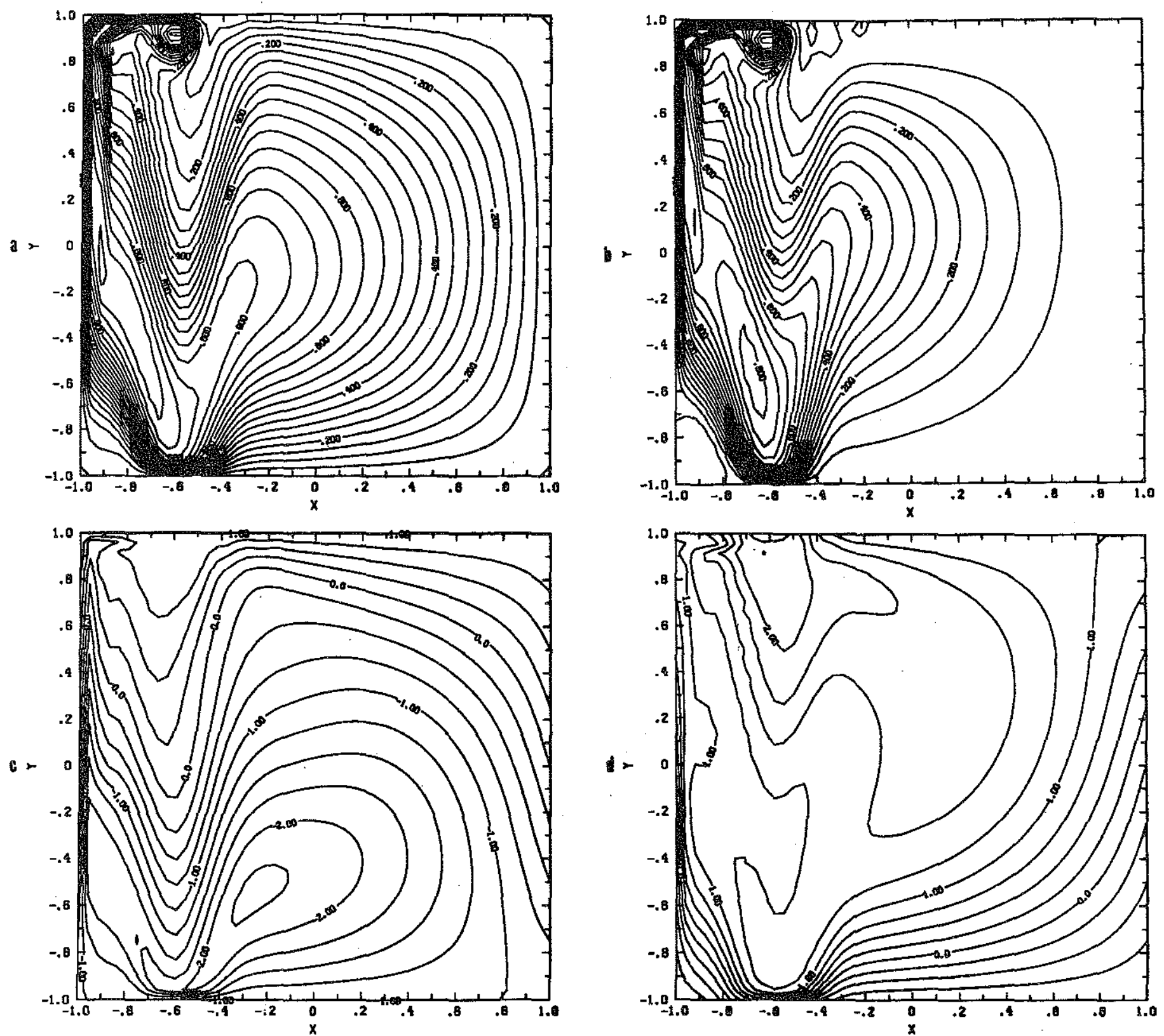

Fig. 3.2. Streamfunction and potential vorticity fields for the experiment R1. a) Upper layer streamfunction. b) Lower layer streamfunction. c) Upper layer potential vorticity. d) Lower layer potential vorticity. Notice the strong flow intensification occuring along the contour $\xi=-0.4$ of Fig. 3.1b. At the same location neither potential vorticity field exhibits a gradient enhancement. 
jump in the streamfunction fields occur. As remarked in the previous chapter, this is because the jump in transport is depth independent and thus does not contribute to the vortex stretching term in the potential vorticity field. Relative vorticity gives a small contribution to the total vorticity field.

Another prediction of the analytical calculation is that in the region west of the ridge the jet continues to flow zonally until it impinges upon the solid western boundary. In the experiment just discussed the ridge is so close to the western boundary of the basin that this effect cannot be revealed.

In the second experiment, R2, I moved the center of the ridge eastward, to the center of the basin, so that $x_{0}=0$. The height was kept the same, $A=1.5$, and so was the halfwidth, $a=0.2$. The other parameters are listed in Table 3.1. The experiment was integrated in time from rest until steady state was reached. The abyssal flow should be confined within the "closed contour" region (the blank region in Fig. 3.1c) and a strong jet should be observed along the characteristic $\xi$ marked by a thick line in Fig. 3.1d. The streamfunction and potential vorticity fields are shown in Fig. 3.3. Again a strong intensification of the gradients occurs in the streamfunction fields (Fig. 3.3a,c), but not in the potential vorticity fields (Fig. 3.3c,d) at the location of a more southern characteristic than that predicted by the inviscid calculation (the thick line in Fig. 3.1d). The inviscid calculation predicts that the vertically integrated difference in transport across the jet is, from (3.2.3), $T=0.77$, in rough agreement with the numerical results, which give a value between 0.8 and 0.9 . In the region west of the ridge $(x \leq-0.3)$, the jet flows almost zonally westward until the western boundary is encountered.

In the last experiment, R3, I tried to assess the effects of viscosity, by keeping all the parameters the same as in R2 except for the diffusive terms, $\kappa$ and $\delta$, which were both increased by a factor of 2. The results are shown in Fig. 3.4 and they should be compared to those shown in Fig. 3.3. As expected, potential vorticity in the lower layer 

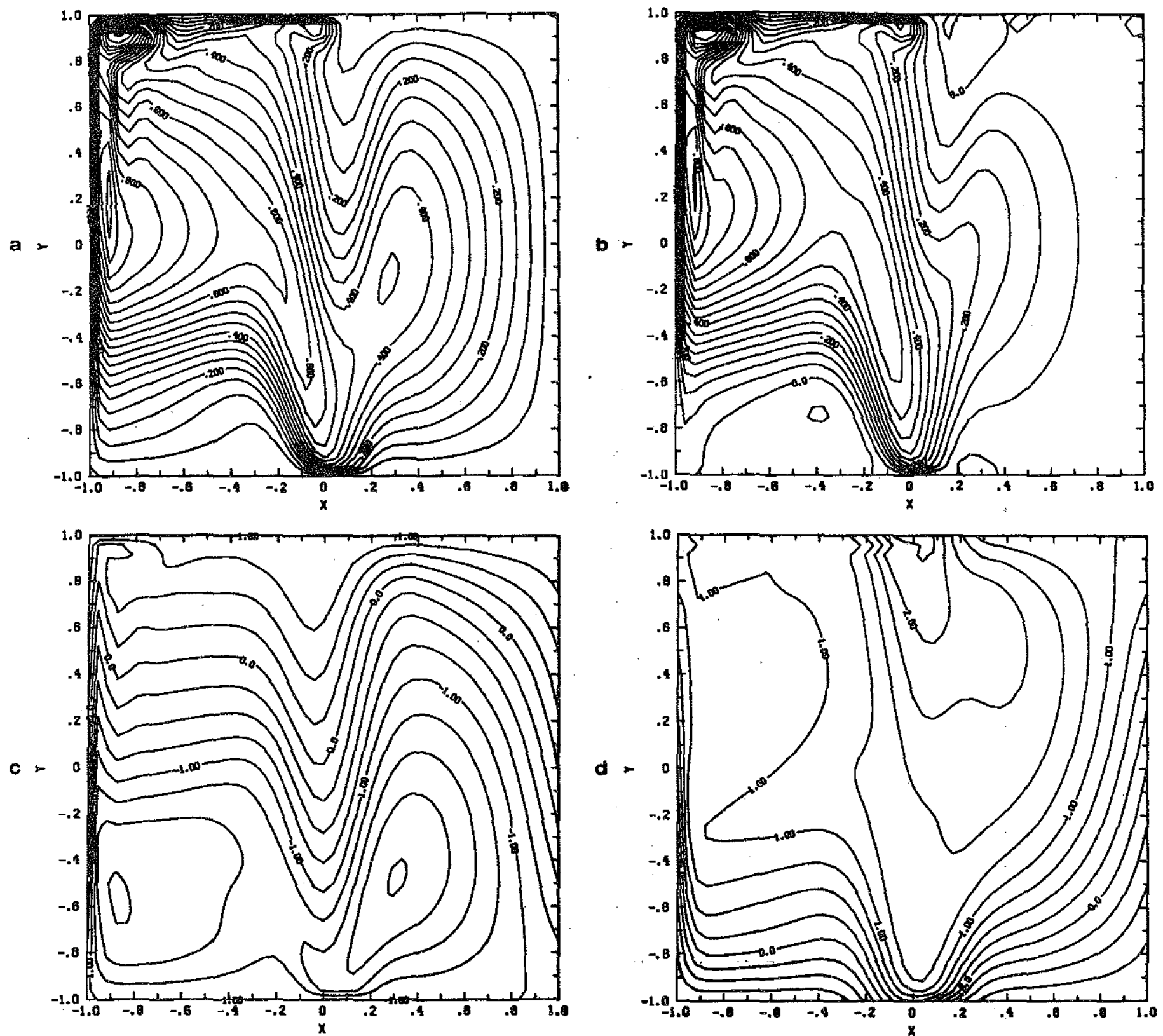

Fig. 3.3. Streamfunction and potential vorticity fields for the experiment R2. a) Upper layer streamfunction. b) Lower layer streamfunction. c) Upper layer potential vorticity. d) Lower layer potential vorticity. Notice the strong flow intensification occuring along the contour $\xi=-0.4$ of Fig. 3.1b. At the same location neither potential vorticity field exhibits a gradient enhancement. The jump in transport across the dividing characteristic is smaller than that occuring in $\mathrm{Rl}$ because the ridge center has been moved westward. 
is less homogeneous, and the flow in the lower layer is weaker. A remarkable feature is that the recirculating gyre, which occured in the northwest corner of the basin in Fig. 3.3 has disappeared and only a mild overshooting of the western boundary current is observed.

Although an increase by a factor of two in the diffusive terms produces some qualitative changes in the overall flow, the analysis of the viscous boundary layer for the vertically integrated flow shows that the jet expands very slowly as diffusion is increased. Calling $\psi=\psi_{1}+\psi_{2}$, the potential vorticity equation for the barotropic flow is

$J(\psi, y+h / 2)+\gamma\left[J\left(\psi_{1}, \nabla^{2} \psi_{1}\right)+J\left(\psi_{2}, \nabla^{2} \psi_{2}\right)\right]=g(x, y)+h_{x} /\left(2 F_{1}\right)+\kappa \gamma \nabla^{4} \psi-\delta \nabla^{2} \psi / 2$

The property that $q_{2}=\delta \psi_{2} / \kappa+$ constant has been used and terms of order $\delta /\left(\kappa F_{2}\right)$ have been neglected. The dominant balance in the region of the jump is given by

$$
J(\psi, y+h / 2)=\gamma \kappa \nabla^{4} \psi-\delta \nabla^{2} \psi / 2
$$

since, for the values of the parameters used, $(\delta / 2)^{1 / 2} \approx(\kappa \gamma)^{1 / 4}$. It turns out that for the experiment R2 lateral diffusion of potential vorticity is bigger than bottom friction, as illustrated in Fig. 3.5, so that the boundary layer width is $O\left((\kappa \gamma)^{1 / 4}\right)$, and the second term of the right hand side of (3.2.4) is subdominant.

In all the experiments presented so far viscosity is quite large and we are quite far from the asymptotic regime discussed in the previous chapter. Indeed the flow in the "blocked" region is substantial and potential vorticity is not very well homogenized in the lower layer. Nevertheless the establishment of an internal jet induced by the ridge-like topography is undeniable even in the experiment $\mathbf{R 3}$ where the maximum Reynolds number $\psi_{2 \max } / \kappa$ is as low as 17 . The numerical experiments thus confirm that, in the presence of bottom topography of varying slope, strong boundary layers are formed in the interior of the ocean. The occurrence of these strong jets is robust to the 

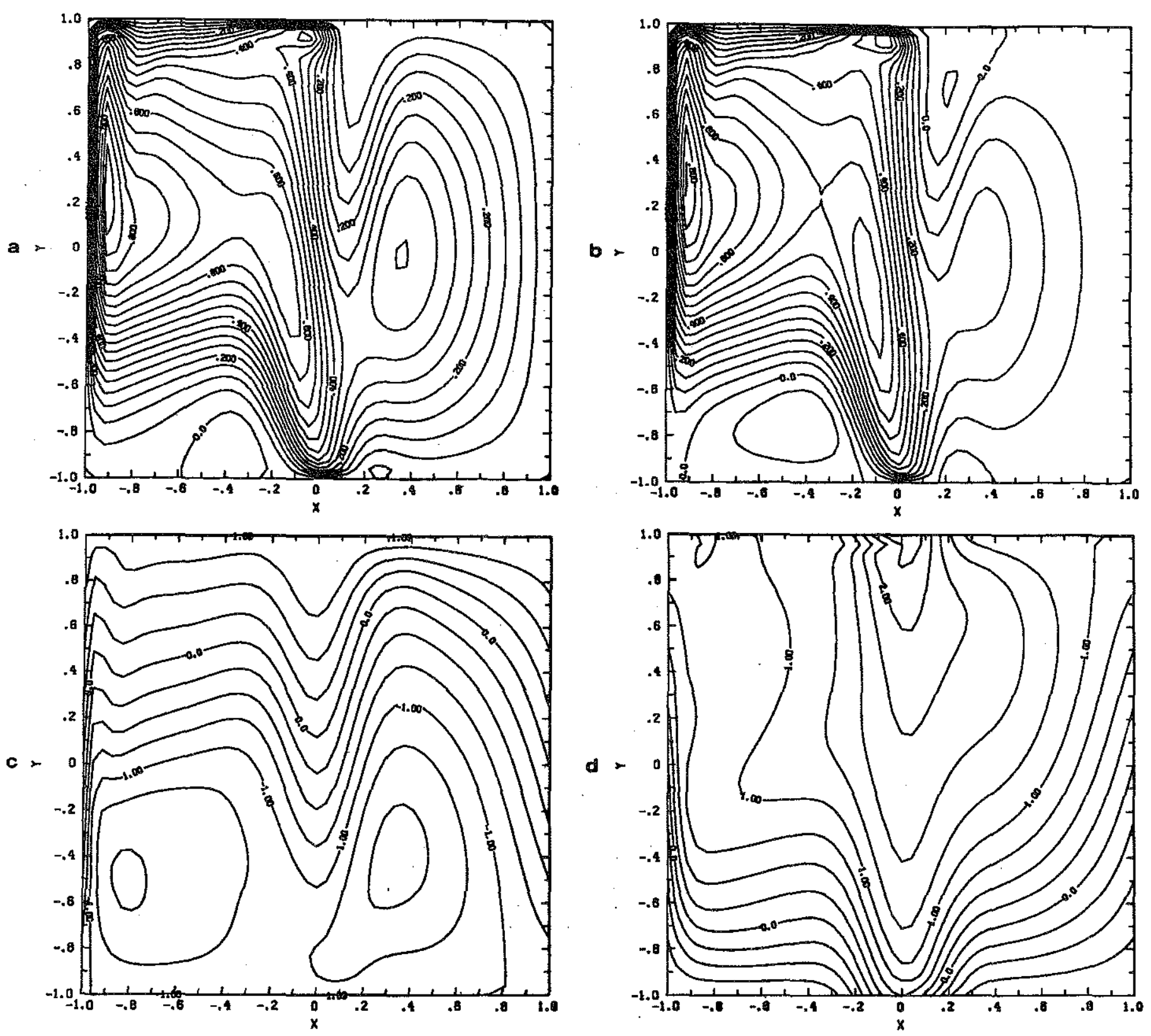

Fig. 3.4. Streamfunction and potential vorticity fields for the experiment R3. a) Upper layer streamfunction. b) Lower layer streamfunction. c) Upper layer potential vorticity. d) Lower layer potential vorticity. An increase by a factor of two in the diffusive terms brings some qualitative changes in the flow pattern: the recirculating gyre appearing in the northwest corner of the basin in Fig. 3.3 has practically disappeared. On the other hand, the internal boundary layer carries the same transport and its width grows very slowly with diffusion. 

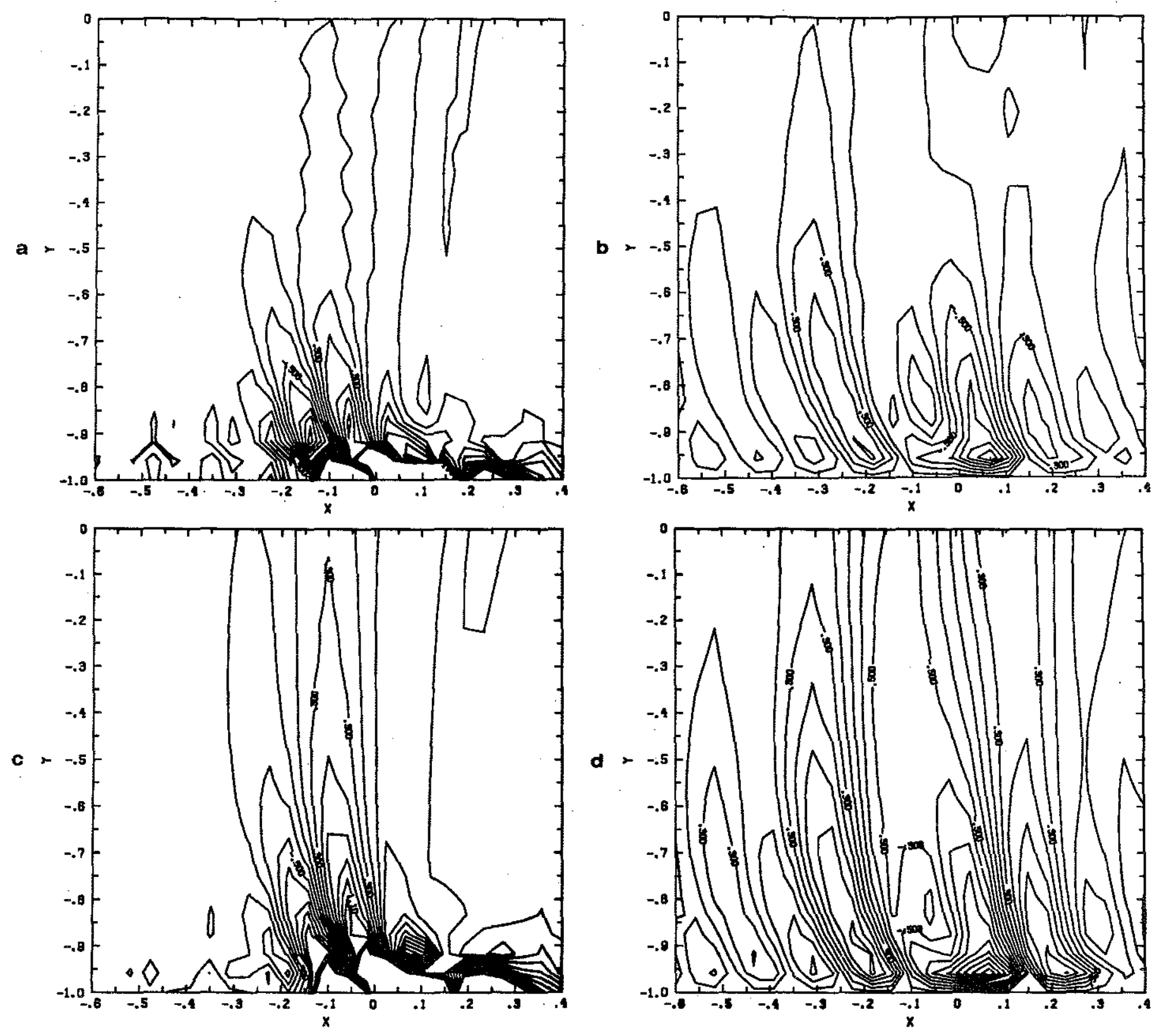

Fig. 3.5. Diffusive terms in the jet region. Notice the change in the $x-y$ scale. a) $\kappa \gamma \nabla^{4}\left(\psi_{1}+\psi_{2}\right)$ for the experiment R2. b) $\delta \nabla^{2} \psi_{2}$ for the experiment R2. c) $\kappa \gamma \nabla^{4}\left(\psi_{1}+\right.$ $\psi_{2}$ ) for the experiment R3. d) $\delta \nabla^{2} \psi_{2}$ for the experiment R3. For the parameters used in R2 lateral diffusion is larger than bottom friction, while for the parameters used in R3 they are of the same size. 
inclusion of viscosity and inertial effects, which simply smooth the jump in transport over a finite width, which grows very slowly as diffusion is increased.

\subsection{Constant slope topography}

In the previous chapter I showed that, in the presence of bottom topography which depends on longitude, strong jets were generated at the boundary of the region of abyssal flow, and this region is well inside the interior of the basin.

In the present series of experiments I force the top layer with the same Ekman pumping used in the previous section, i.e. $g(x, y)=-\cos (\pi y / 2)$, but the bottom topography is of the form $h b=-\alpha x$, with $\alpha>0$. The values of the parameters used are summarized in Table 3.2. The inviscid calculation predicts that the lower layer should be in motion only in the region of "closed contours" (the empty region in Fig. 3.6), and that an internal boundary layer should be observed at the location marked by a thick solid segment in Fig. 3.6. The streamfunction and potential vorticity fields resulting after time integration from rest of the first experiment, S1, are shown in Fig. 3.7. Although the maximum Reynolds number is $\psi_{2 \max } / \kappa=50$ and thus quite large, there is a weak abyssal flow in the region where the inviscid calculation suggests that there should be no motion. Even though the abyssal, diffusively driven flow is weak, because the topographic slope is so large, it changes the barotropic transport substantially and the topographically induced velocity enhancement occurs on the southern solid boundary rather than in the interior. The barotropic transport $\psi=\psi_{1}+\psi_{2}$ is governed, to first order, by

$$
\psi_{x}+\alpha \psi_{y}=-\cos (\pi y / 2)
$$

In the "blocked" region, south of where the jet should be observed $(y \approx-0.8)$, the wind forcing is weak, and the second term on the left hand side, which was neglected in the inviscid calculation, becomes as big as the Ekman pumping. A change in the barotropic flow in the southwest corner of the basin enlarges the "closed contour" region enough 

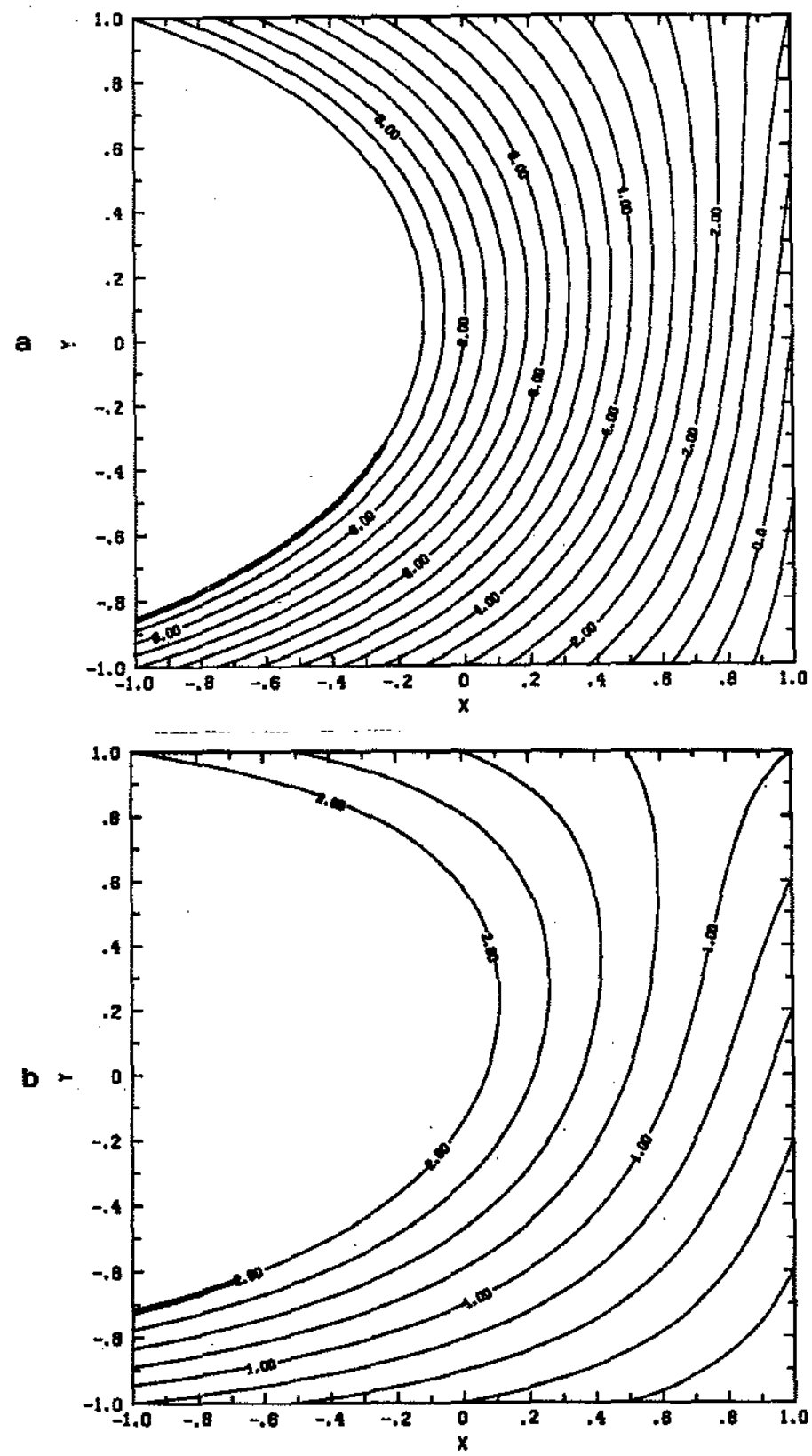

Fig. 3.6. a) Lower layer potential vorticity contours, as predicted by the inviscid calculation, for the parameters used in $\mathbf{S 1}$. The abyssal flow should be confined in the region where no contours are drawn ("closed contours"), and $q_{2}-\delta / \kappa \psi_{2}$ should be constant there. b) Same as Fig. 3.6a, but for the parameters used in S2. The internal jets should be observed along the thick curve segments. 
so as to include the southern boundary (compare Fig. 3.7d with Fig. 3.6a) and the boundary layer occurs on the solid wall.

\begin{tabular}{|c|c|c|c|c|c|}
\hline Experiment & $\alpha$ & $\gamma$ & $\kappa$ & $\delta$ & $F_{1}=F_{2}$ \\
\hline S1 & 4.0 & 0.0005 & 0.012 & 0.006 & 4 \\
S2 & 0.8 & 0.0005 & 0.018 & 0.009 & 2 \\
\hline
\end{tabular}

Table 3.2: Summary of the parameters used for the experiments in the presence of a constant slope topography of the form $h b=-\alpha x$ and of an Ekman pumping of the form $g(x, y)=-\cos (\pi y / 2)$. All the quantities are nondimensional.

For the second experiment, S2, the region of abyssal flow, as predicted by the inviscid calculation, is shown in Fig. 3.6b. Now the topographic slope has been reduced by a factor of five. The streamfunction and potential vorticity fields resulting from the numerical integration are shown in Fig. 3.8. No sign of velocity intensification is evident in the streamfunction field except in the western boundary layer and in the recirculation region (northwest corner). The potential vorticity contours in the lower layer are quite different than those predicted by the inviscid calculation (Fig. 3.6b), the difference being larger near the outermost closed $q_{2}$ contour. To assess the effects of viscosity I calculated the viscously driven abyssal flow in the "blocked contour" region. In this region the lower layer flow is weak and to first order the upper layer flow is in Sverdrup balance, while the lower layer is forced by the weak stresses induced by the upper layer motion

$$
\begin{gathered}
J\left(\psi_{1}, y\right)=-\cos (\pi y / 2) \\
J\left(\psi_{2}, y+h+F_{2} \psi_{1}\right)=\kappa \nabla^{2} q_{2} \simeq \kappa \nabla^{2} \psi_{1}
\end{gathered}
$$

with boundary conditions $\psi_{2}=0$ on $x=1, y=1$. The solution of this linear problem is shown in Fig. 3.9a. The viscously driven flow is indeed maximum in the region where the topographically induced jet should be observed. The amplitude, though, is about a factor of five smaller than that observed in the numerical experiment. The reason for 

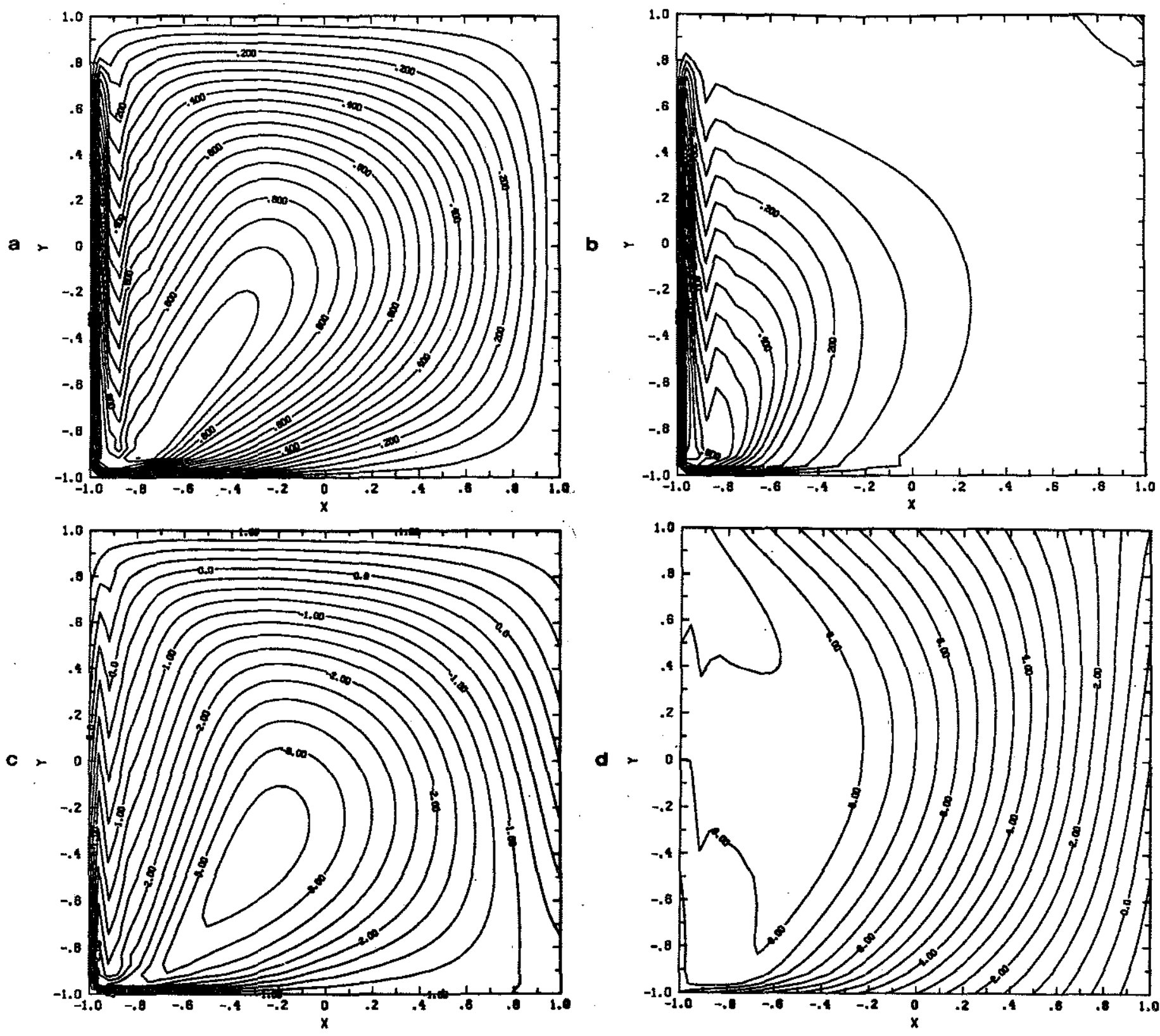

Fig. 3.7. Streamfunction and potential vorticity fields for the experiment S1. a) Upper layer streamfunction. b) Lower layer streamfunction. c) Upper layer potential vorticity. d) Lower layer potential vorticity. The flow is closed by western and southern boundary layers both occurring on solid walls. The region of homogeneous potential vorticity differs from that shown in Fig. 3.6a mostly in the southwest corner. 

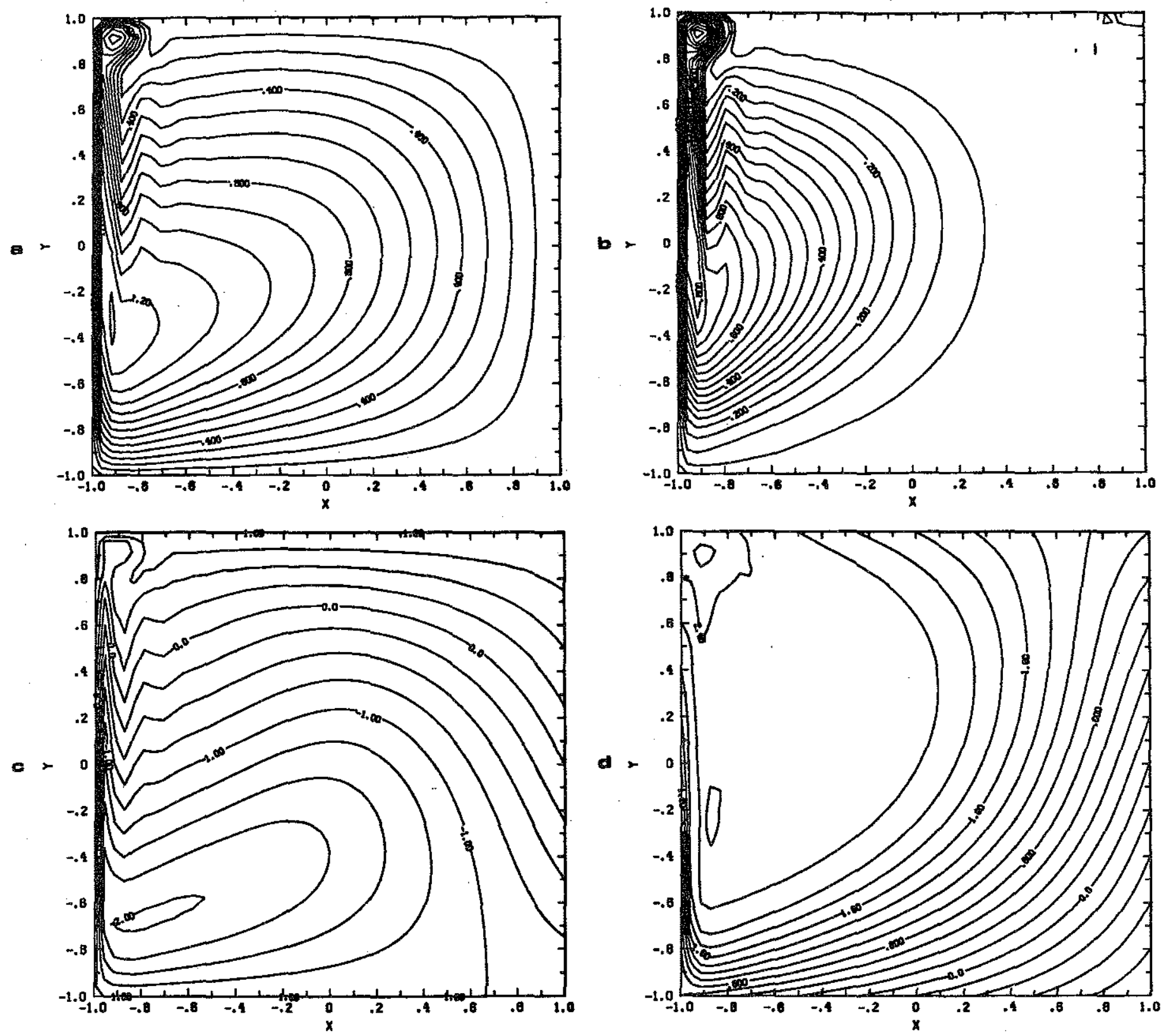

Fig. 3.8. Streamfunction and potential vorticity fields for the experiment S2. a) Upper layer streamfunction. b) Lower layer streamfunction. c) Upper layer potential vorticity. d) Lower layer potential vorticity. The lower layer moves also in the "blocked" region avoiding the onset of an internal boundary layer. The $q_{2}$ field is quite different from that shown in Fig. 3.6b. For example the contour $q_{2}=2.4$ has moved from the "blocked" region to the "closed" region. 
this discrepancy is that, in the linear calculation (3.3.1) the strong recirculating gyre occurring in the northwest corner of the basin has not been modelled. Specifically the contribution of the recirculating gyre to the source term $\left(\kappa \nabla^{2} q_{2}\right)$ in (3.3.1) is substantial as shown in Fig. 3.9b. Because the $q_{2}$ contours close to the region where the jet should be observed trace back to the northwest corner of the basin, crossing the "wake" of the recirculation, this localized source affects the whole region near the outermost closed $q_{2}$ contour.

The presence of the viscously driven flow in turn deforms the lower layer potential vorticity contours in the interior so that they become parallel to the characteristics given in (2.2.6), thus avoiding the jump in transport described in the previous chapter, Section 2.2. The reason why a moderate amount of friction and inertia has such a devastating effect on the prediction of the inviscid calculation is the following. In the inviscid theory it is crucial that the characteristics (2.2.6) intersect the boundary of the abyssal flow twice in the interior. If the distance between the two points of intersection is small the jump in transport will be small. Inspection of Fig. 3.6b shows that it is enough to deform the outermost closed $q_{2}$ contour by a small amount to avoid the abovementioned intersection. On the other hand in Section 2.2 it was shown that, if the Ekman pumping is such that the flow can be closed in the interior (see Fig. 2.3) then the jump in transport will occur on half of the boundary of the region of abyssal flow (the northwest half of the circle of radius $r_{1}$ in Fig. 2.3).

With this in mind a series of experiments was performed with an Ekman pumping of the form $g(x, y)=-(\pi / 2) \sin (\pi x / 2) \cos (\pi y / 2)$. In the absence of lower layer flow the upper layer is in Sverdrup balance, $\psi_{1}=\cos (\pi x / 2) \cos (\pi y / 2)$, and the circulation can be closed without appending boundary layers. In the absence of western boundary currents, even in the limit of very weak dissipation, there is no need for a recirculating gyre in order to achieve the steady state (see Chapters 4 and 5). In the experiments 

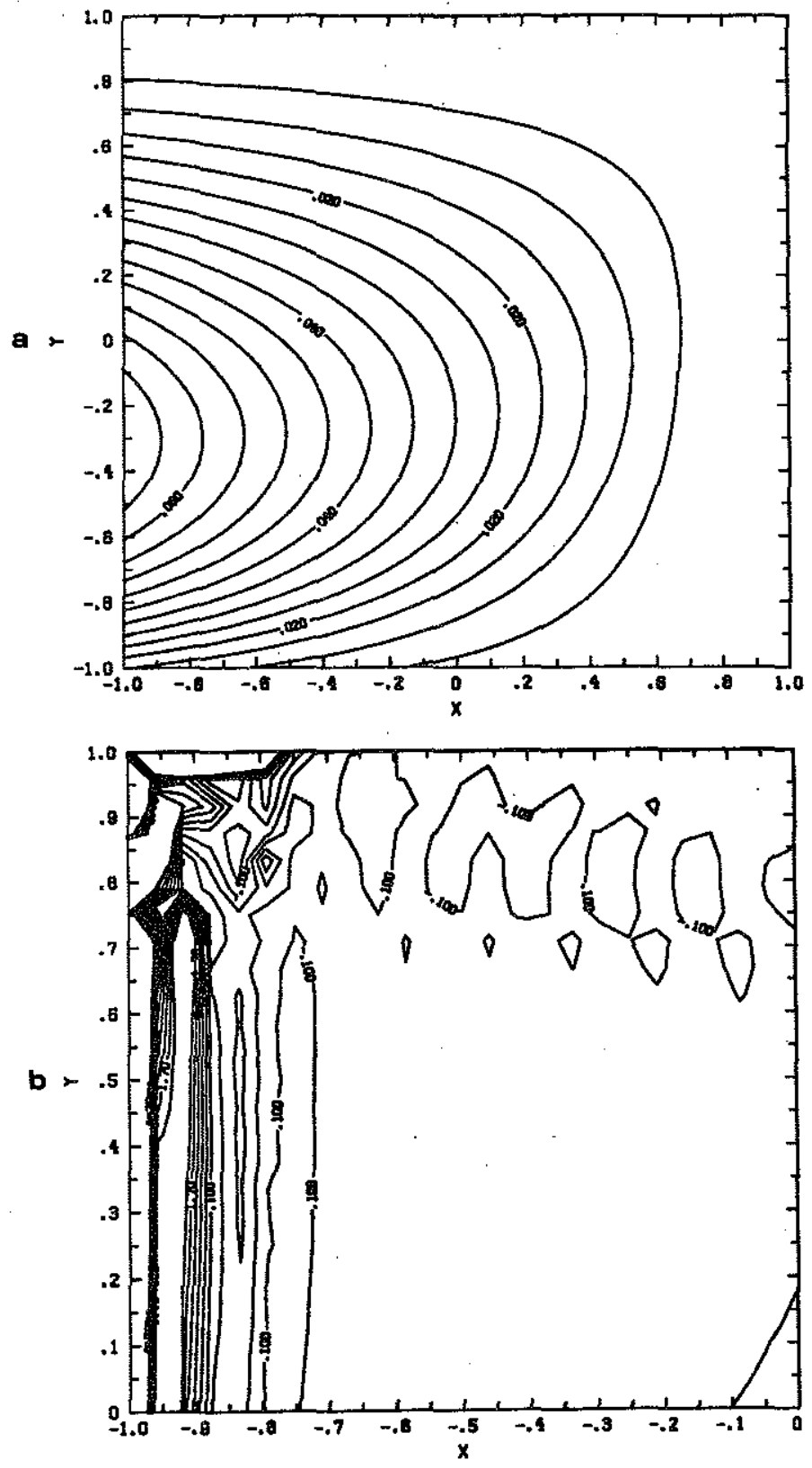

Fig. 3.9. a) Viscously driven lower layer flow resulting from the solution of (3.3.1b) for the parameters used in S2. The amplitude of the solution is too small. b) Contours of $\kappa \nabla^{2} q_{2}$ in the northwest half of the basin. In the recirculation region $\kappa \nabla^{2} q_{2}$ is very big and such a region extends eastward in the interior. 


\begin{tabular}{|c|c|c|c|c|c|}
\hline Experiment & $\alpha$ & $\gamma$ & $\kappa$ & $\delta$ & $F_{1}=F_{2}$ \\
\hline C1 & 0.0 & 0.0005 & 0.0125 & 0.006 & 4 \\
C2 & 0.5 & 0.0005 & 0.0125 & 0.006 & 4 \\
C3 & 2.0 & 0.0005 & 0.0125 & 0.006 & 4 \\
\hline
\end{tabular}

Table 3.3: Summary of the parameters used for the experiments in the presence of a constant slope topography of the form $h b=-\alpha x$ and of an Ekman pumping of the form $g(x, y)=-\pi / 2 \sin (\pi x / 2) \cos (\pi y / 2)$. All the quantities are nondimensional.

presented the topography was chosen as $h b=-\alpha x, \alpha>0$, the values of the parameters used are summarized in Table $\mathbf{3 . 3}$.

In the first experiment, $\mathrm{C} 1$, the bottom is flat, i. e. $\alpha=0$. In the limit of infinitesimal viscosity the lower layer should move only inside the region bounded by the thick line in Fig. 3.10a, keeping the quantity $q_{2}-\delta / \kappa \psi_{2}$ constant and equal to 1 . The streamfunction and potential vorticity fields obtained by time integration from rest are shown in Fig. 3.11, together with the $q_{2}-\delta / \kappa \psi_{2}$ field. Although the region of "blocked" $q_{2}$ contours in the numerical experiment is wider than that predicted by the inviscid calculation, the abyssal flow occupies the whole basin, so that the circulation is much more vertically coherent than expected. Moreover the homogenized value of $q_{2}-\delta / \kappa \psi_{2}$ is not 1 , but $\approx 0.45$ (the range of values being between -1 . and 1 .). The maximum Reynolds number for this experiment is $\psi_{2} / \kappa=34$, but the Reynolds number in the region of "blocked" contours is $\approx 8$. The effect of viscosity, in the absence of topography, is to shrink the region of homogenized $q_{2}-\delta / \kappa \psi_{2}$, and yet to expand the region of abyssal flow. However, in the absence of bottom topography, the vertically integrated flow is, to first order, independent of the motion of the lower layer, so that the inviscid prediction for the barotropic flow is not invalidated by the inclusion of dissipative and inertial effects. In other words diffusion acts mostly on the baroclinic mode, leaving the vertically integrated flow almost unchanged. 

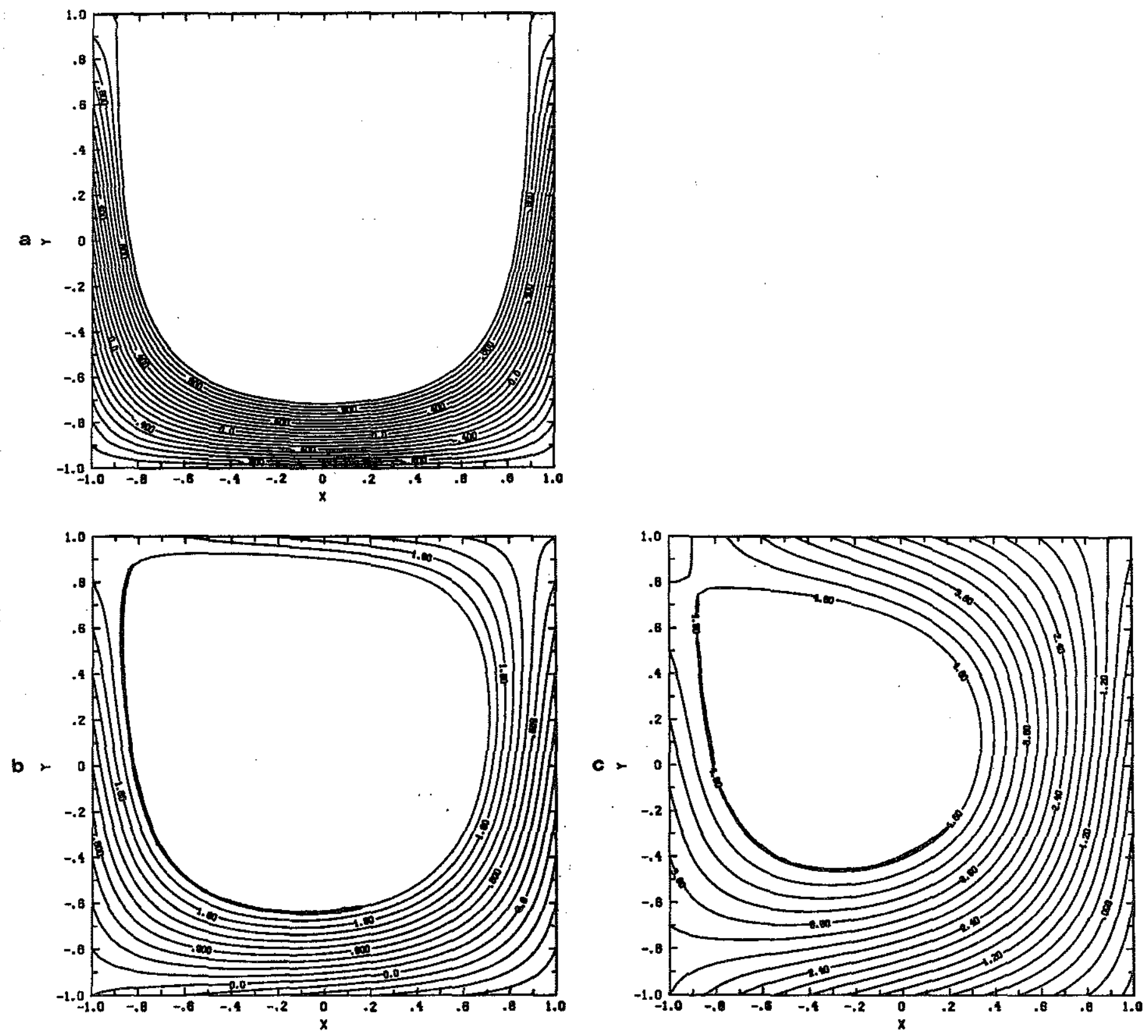

Fig. 3.10. a) Lower layer potential vorticity contours, as predicted by the inviscid calculation, for the parameters used in $\mathrm{C} 1$. The abyssal flow should be confined in the region where no contours are drawn ("closed contours"), and $q_{2}-\delta / \kappa \psi_{2}$ should be constant there. b) Same as Fig. 3.10a, but for the parameters used in C2. c) Same as Fig. 3.10b, but for the parameters used in C3. The internal jets should be observed along the thick curve segments. 

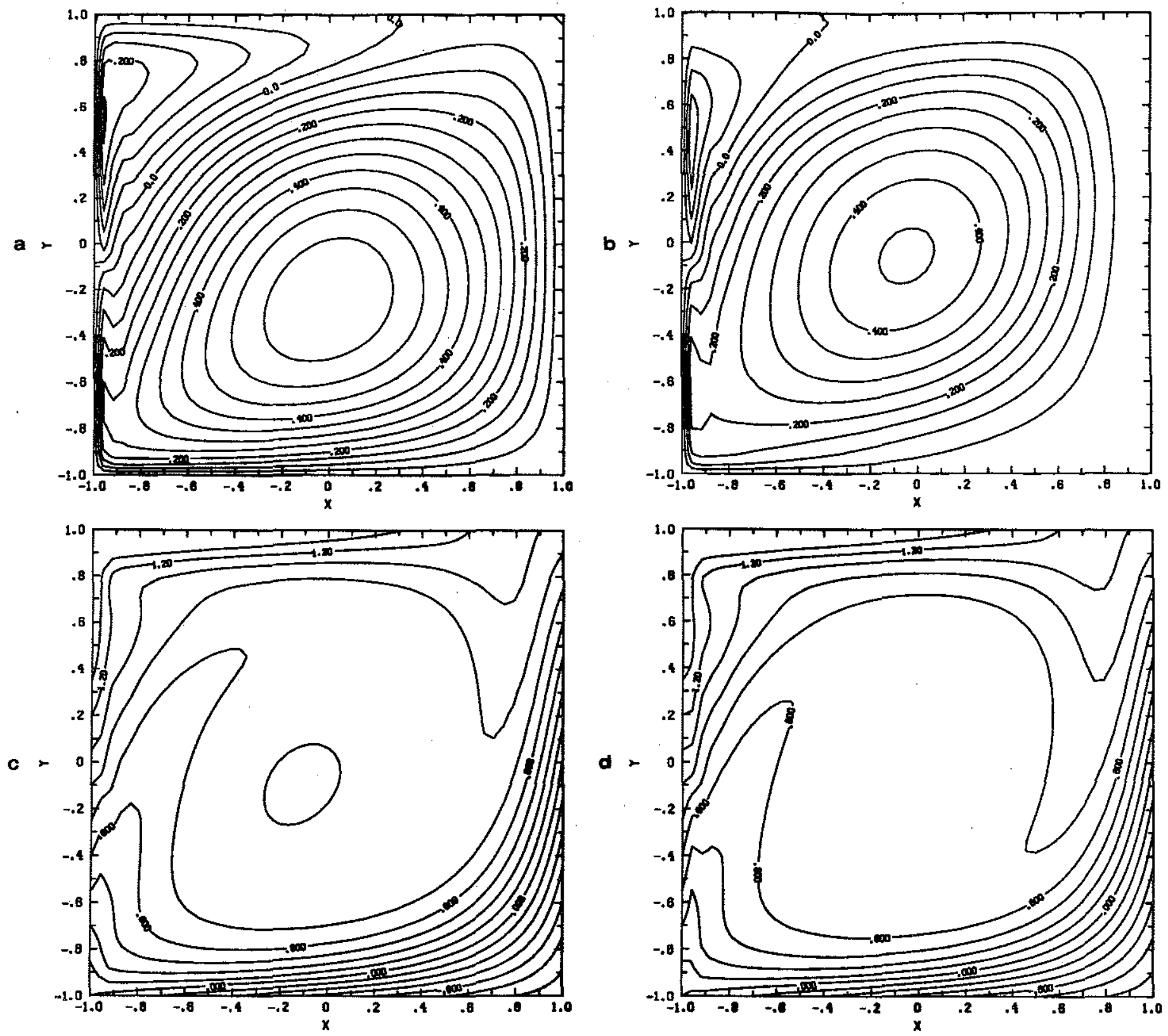

Fig. 3.11. Streamfunction and potential vorticity fields for the experiment $\mathrm{C} 1$. a) Upper layer streamfunction. b) Lower layer streamfunction. c) Lower layer potential vorticity. d) $q_{2}-\delta / \kappa \psi_{2}$. Although the region of homogenized $q_{2}-\delta / \kappa \psi_{2}$ is smaller than that shown in Fig. 3.10a the abyssal flow occupies a larger region. 
In the presence of topography the barotropic flow cannot be calculated without knowledge of the abyssal flow, even if the viscous and inertial effects are neglected. In the next experiment of the series, C2, I kept all the parameters the same as in C1 except for the bottom slope which was set to $\alpha=0.5$. The lower layer potential vorticity contours in the absence of abyssal flow are shown in Fig. 3.10b. The inviscid theory of Section 2.2 predicts a discontinuity in transport on the southwest half of the abyssal flow boundary marked by a thick line in Fig. 3.10b. The streamfunction and potential vorticity fields, resulting from time integration of $\mathrm{C} 2$ using the steady state reached in $\mathrm{C} 1$ as initial condition, are shown in Fig. 3.12. Again there is a weak abyssal flow in the region of blocked $q_{2}$ contours so that the lower layer moves everywhere in the basin. The homogenized value of $q_{2}-\delta / \kappa \psi_{2}$ is $\approx 0.7$ instead of the value 2.0 as given in Fig. 3.10b. Unlike the flat bottom experiment, $\mathrm{C} 1$, the weak, viscously driven flow induces qualitative changes in the flow. The inviscid calculation predicts an anticyclonic circulation in both layers, while in C2 a weak cyclonic gyre appears in both layers, confined to the northwest corner of the basin. This cyclonic gyre is not equivalent to the recirculating gyre appearing at the same location in the experiment S2, but is a feature solely due to topography. The circulation pattern observed in C2 is very similar to the one that would be obtained neglecting viscous and inertial effects in a barotropic model with the same bottom topography. For one layer the flow is described by

$$
J(\psi, y-\alpha x)=-\pi / 2 \sin (\pi x / 2) \cos (\pi y / 2)
$$

with boundary conditions $\psi=0$ on $x=1, y=1$. The solution of (3.3.2) is shown in Fig. 3.13a, and is qualitatively very similar to the vertically integrated flow resulting from C2 (Fig. 3.13b). The question is: what drives the cyclonic circulation in the lower layer?

In Fig. 3.14 I have plotted the balance of terms in the lower layer as a function of $x$, for the two latitudes $y=0.8$ and $y=0.6$. At the northern latitude, where the cyclonic 

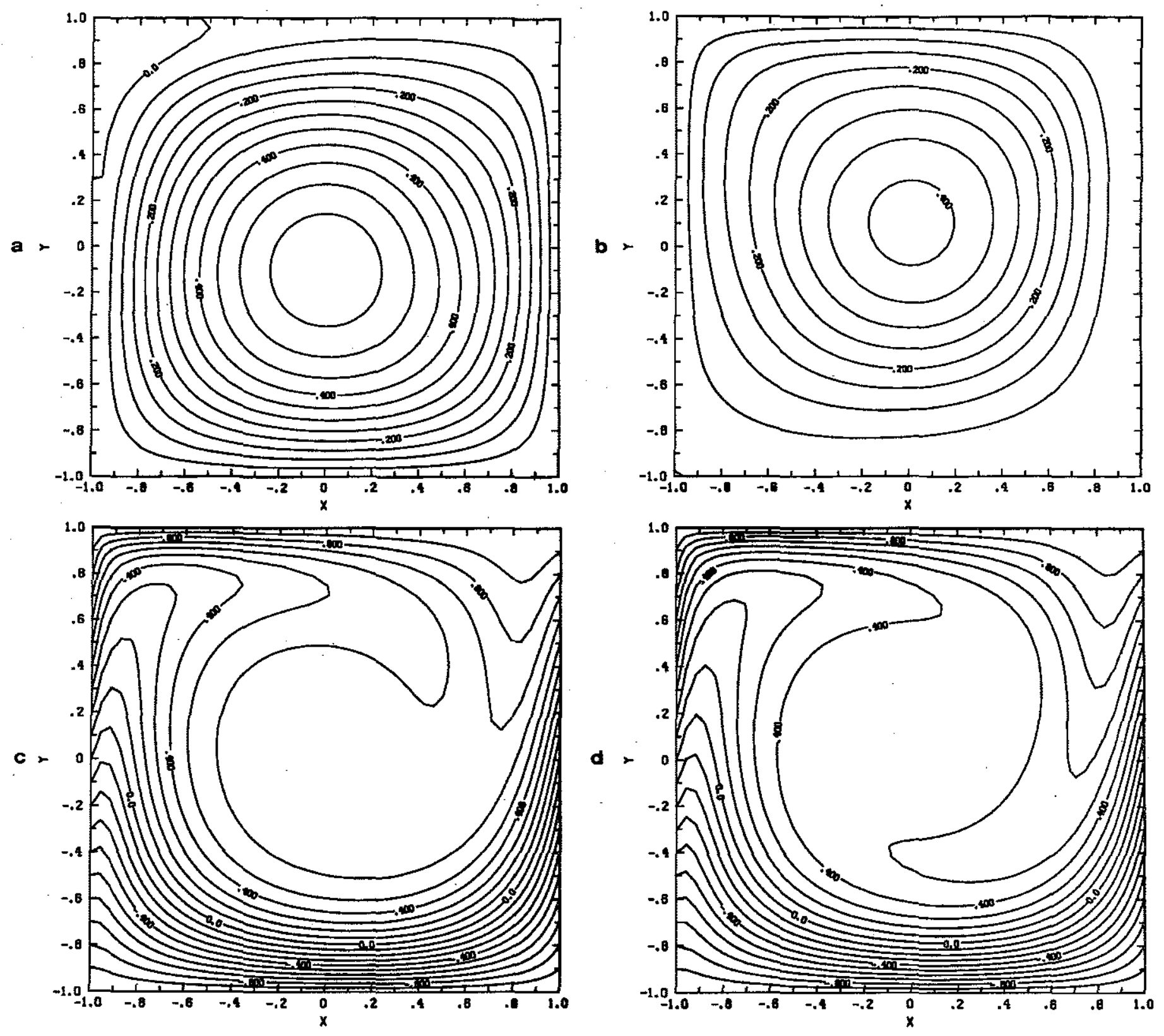

Fig. 3.12. Streamfunction and potential vorticity fields for the experiment C2. a) Upper layer streamfunction. b) Lower layer streamfunction. c) Lower layer potential vorticity. d) $q_{2}-\delta / \kappa \psi_{2}$. The region of homogenized $q_{2}-\delta / \kappa \psi_{2}$ is smaller than that shown in Fig. 3.10b and yet the abyssal flow occupies the whole basin. 

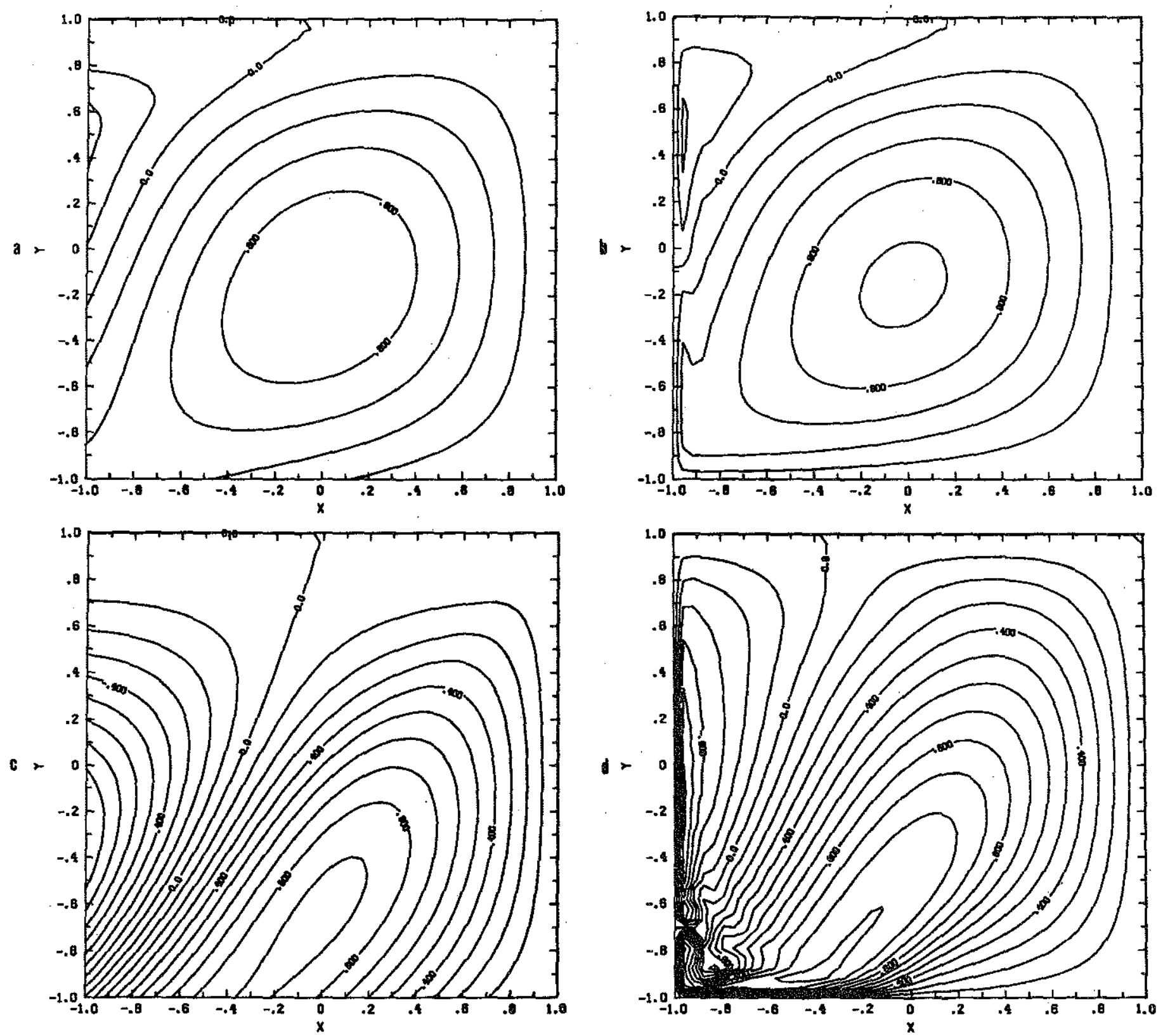

Fig. 3.13. a) Solution of the barotropic linear problem (3.3.2) with an equivalent bottom topography $h=-\alpha x / 2, \alpha=.5$. b) Barotopic flow $\psi_{1}+\psi_{2}$ for the esperiment C2. c) Same as Fig. 3.13a for $\alpha=1.0$. d) Same as Fig. 3.13b for the experiment C3. 
gyre is observed, the interfacial friction term (curve A in Fig. 3.14b) dominates and is bigger than each single term in the Jacobian term (Fig. 3.14a), so that the dominant balance in the lower layer is

$$
J\left(\psi_{2}, y+h+F_{2} \psi_{1}\right)=\kappa F_{2} \nabla^{2}\left(\psi_{1}-\psi_{2}\right)
$$

At the southern latitude viscosity is much less important and, in the interior, the dominant balance is $J\left(\psi_{2}, y+h+F_{2} \psi_{1}\right)=0$ as assumed in the inviscid calculations.

The dominance of the interfacial friction in the northwest corner of the basin locks the two layer together and this is why the flow resembles the linear barotropic solution shown in Fig. 3.13a. In most of the basin friction is still negligible and the maximum Reynolds number is $\psi_{2 \max } / \kappa=40$. Unlike the flat bottom experiment, a change in the flow in the lower layer from the inviscid prediction can induce a substantial change in the barotropic transport. For this experiment I have checked that the dominant balance for the depth integrated flow is, except in the western and southern boundary layers,

$$
\left(\psi_{1}+\psi_{2}\right)_{x}+\alpha \psi_{2 y}=-(\pi / 2) \sin (\pi x / 2) \cos (\pi y / 2)
$$

Because of the second term on the left hand side, the behavior of the barotropic flow can be changed by the motion in the lower layer.

In the last experiment presented, C3, I kept all the parameters the same as in C2 except for the topographic slope, which was increased by a factor of 4 . The boundary of the region of abyssal flow predicted by the inviscid calculation is shown in Fig. 3.10c and the discontinuity in transport should occupy the south-west half of the "egg" shaped boundary. The streamfunction and potential vorticity fields resulting after time integration from rest are shown in Fig. 3.15. Again a cyclonic gyre, stronger than that obtained in $\mathrm{C} 2$, appears in the western portion of the basin. Where the bulk of the abyssal flow occurs, the lower layer potential vorticity is homogenized, but the actual region is very different from that predicted by the inviscid theory. The abyssal 

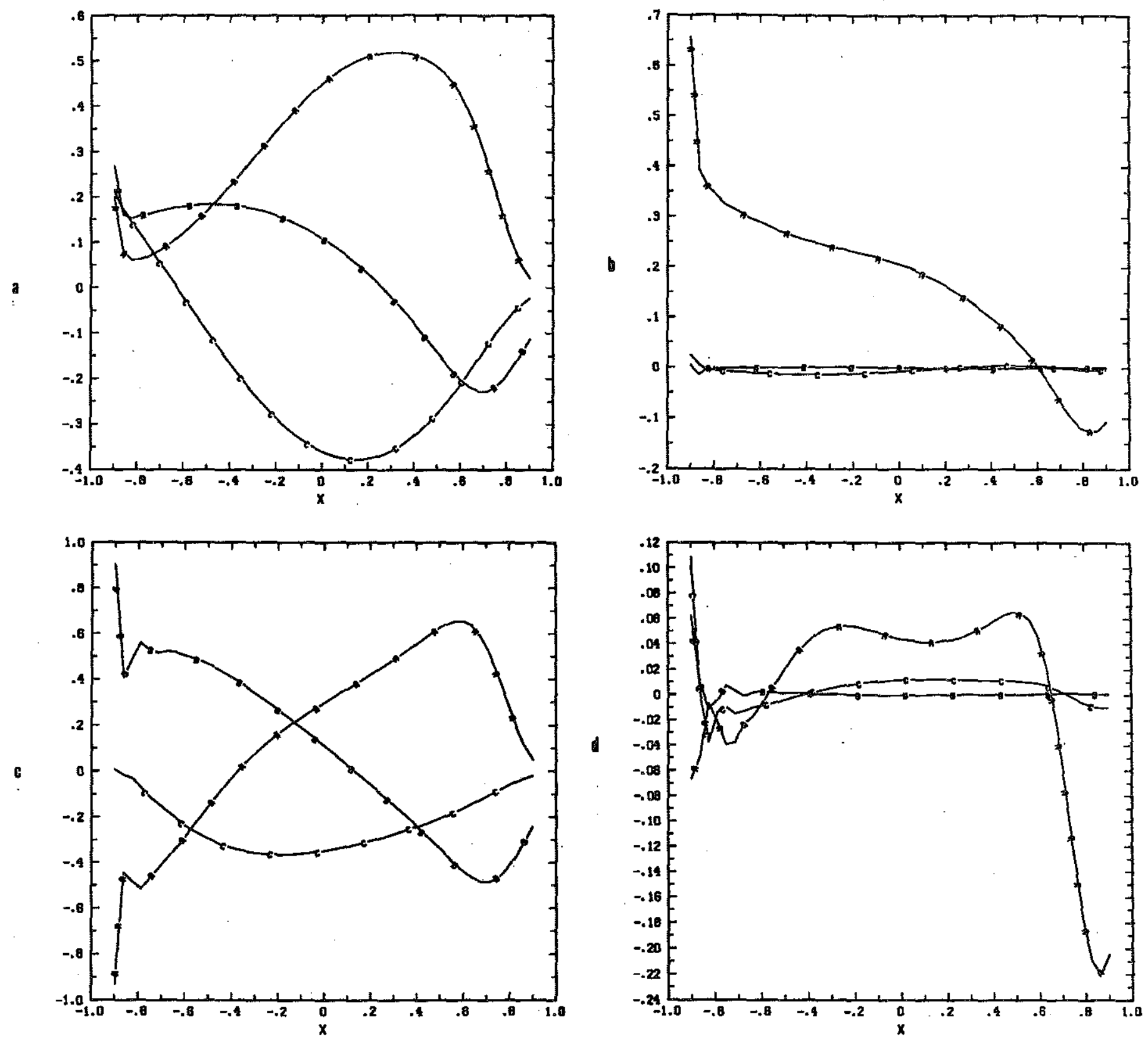

Fig. 3.14. Balance of terms in the lower layer potential vorticity for the experiment C2, as a function of $x$, at fixed latitudes. a) Advective terms at $y=0.8$ : curve $\mathrm{A}=J\left(\psi_{2}, F_{2} \psi_{1}\right)$, curve $\mathrm{B}=J\left(\psi_{2}, y\right)$, curve $\mathrm{C}=J\left(\psi_{2}, h\right)$. b) Viscous and inertial terms at $y=0.8$ : curve $\mathrm{A}=\kappa \nabla^{2} q_{2}$, curve $\mathrm{B}=J\left(\psi_{2}, \gamma \nabla^{2} \psi_{2}\right)$, curve $\mathrm{C}=\delta \nabla^{2} \psi_{2}$. c) Advective terms at $y=0.6$ : curve $\mathrm{A}=J\left(\psi_{2}, F_{2} \psi_{1}\right)$, curve $\mathrm{B}=J\left(\psi_{2}, y\right)$, curve $\mathrm{C}=J\left(\psi_{2}, h\right)$. c) Viscous and inertial terms at $y=0.6$ : curve $A=\kappa \nabla^{2} q_{2}$, curve $B=J\left(\psi_{2}, \gamma \nabla^{2} \psi_{2}\right)$, curve $\mathrm{C}=\delta \nabla^{2} \psi_{2}$. 
flow invades the southwestern portion of the basin and the vertically integrated flow resembles closely the one layer inviscid solution of Eq. (3.3.2), shown in Fig. 3.13c.

In the following I will try to explain why in the presence of small interfacial friction and topography the potential vorticity and flow field are qualitatively different from those predicted by the inviscid theory. If the lower layer were, to first order at rest in the northwest corner of the basin, then, to $O(\kappa)$, the lower layer would move according to

$$
J\left(\psi_{2}, \hat{q}_{2}\right)=-\kappa F_{2} \pi^{2} / 2 \cos (\pi x / 2) \cos (\pi y / 2)
$$

where $\hat{q}_{2}=y-\alpha x+F_{2} \cos (\pi x / 2) \cos (\pi y / 2)$. The $\hat{q}_{2}$ contours are shown in Fig. 3.10c. Therefore the viscously induced lower layer flow will be northeastward to the east of the outermost $\hat{q}_{2}$ contour and south-westward to the west. The effect of this weak abyssal flow on the depth integrated transport is, taking the $x$ derivative of Eq. (3.3.4), to induce a positive relative vorticity, which means a cyclonic circulation. The reasoning depicted here is only heuristic, since near the northern boundary the Ekman pumping is small and its contribution may not exceed the viscous term. Indeed this is the reason why even if the viscously induced flow is weak, the $q_{2}$ contours in Fig. 3.15d in the northwest corner of the basin are so different than those in Fig. 3.10c.

In Fig. 3.16 I have plotted the balance of terms in the lower layer for the experiment C3, as a function of $x$ at two fixed latitudes, $y=0.85$ and $y=0.50$. At the northern latitude the interfacial friction term is dominant and the balance is as in Eq. (3.3.3), while at the southern latitude the viscous terms are subdominant. Because the flow at the northwest corner is weak (being near the zero of the Ekman pumping) the local Reynolds number is small. Therefore the weak viscously induced flow is as big as the wind driven flow and it is able to deform the lower layer potential vorticity contours substantially: in Fig. 3.10c their slope is opposite to that in Fig. 3.15c. The geometry of the potential vorticity contours in the northwest corner is essential to the separation 

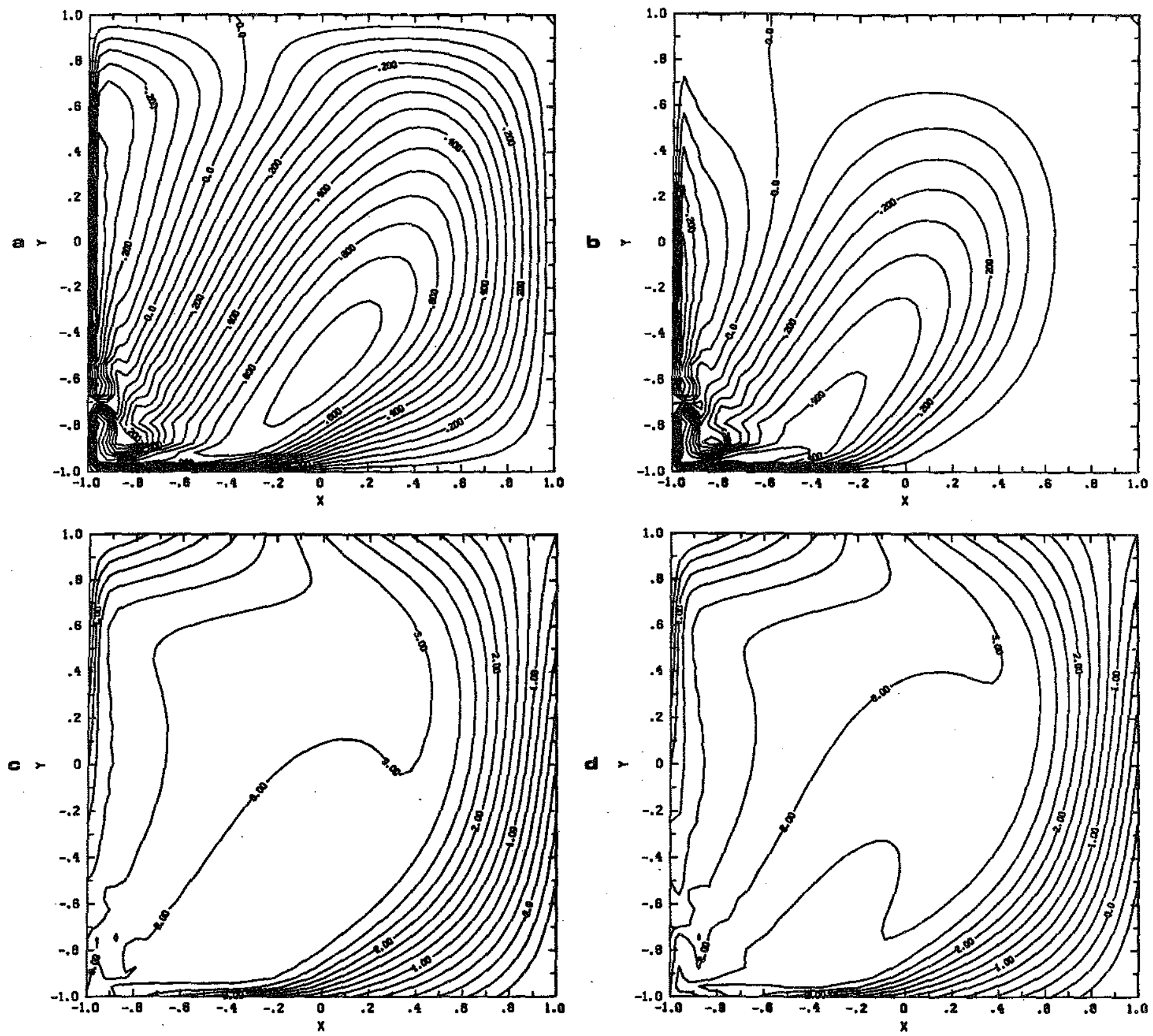

Fig. 3.15. Streamfunction and potential vorticity fields for the experiment C3. a) Upper layer streamfunction. b) Lower layer streamfunction. c) Lower layer potential vorticity, d) $q_{2}-\delta / \kappa \psi_{2}$. The region of homogenized $q_{2}-\delta / \kappa \psi_{2}$ is completely different from that shown in Fig. 3.10c. 
of "closed" contours from "blocked" contours and a change in this region affects the whole abyssal flow.

I should point out that the experiment just discussed is the most inviscid I could obtain with the given resolution. At lowest viscosities strong instabilities, generated by the recirculating gyre appearing in the southwest corner of Fig. 3.15a,b rendered the problem numerically intractable.

\subsection{Summary and discussion}

In the previous chapter it has been shown that, in the presence of $x$-dependent topography, strong internal boundary layers are found when viscous and inertial effects are neglected. Two types of jets were discussed. One occurs in the presence of topography of varying slope and arises both in vertically homogeneous and stratified models. The other occurs even in the presence of constant slope topography as long as the bottom elevation depends on longitude. The latter internal boundary layer is found only in baroclinic gyres, although it has a component which is depth independent.

In order to test the robustness of the results I included the effects of lateral and interfacial friction as well as of inertia. The problem was solved numerically by time integration until the steady state was reached. The internal jets, connecting the southern to the western boundary layers in the presence of a ridge-like topography, found in the numerical model agreed with the results of the inviscid theory. Consistent with the inviscid prediction, the potential vorticity field doesn't exhibit the increase in gradient observed in the streamfunction field at the jet location. The vertically integrated transport carried by the jets was found to be in quantitative agreement with the analytical calculation. The occurrence of the internal boundary layer was shown to survive moderate increases in the diffusion coefficient and the jet width grows very slowly when $\kappa$ is increased. This indicates that the inviscid result is robust to the inclusion of weak nonconservative effects. 

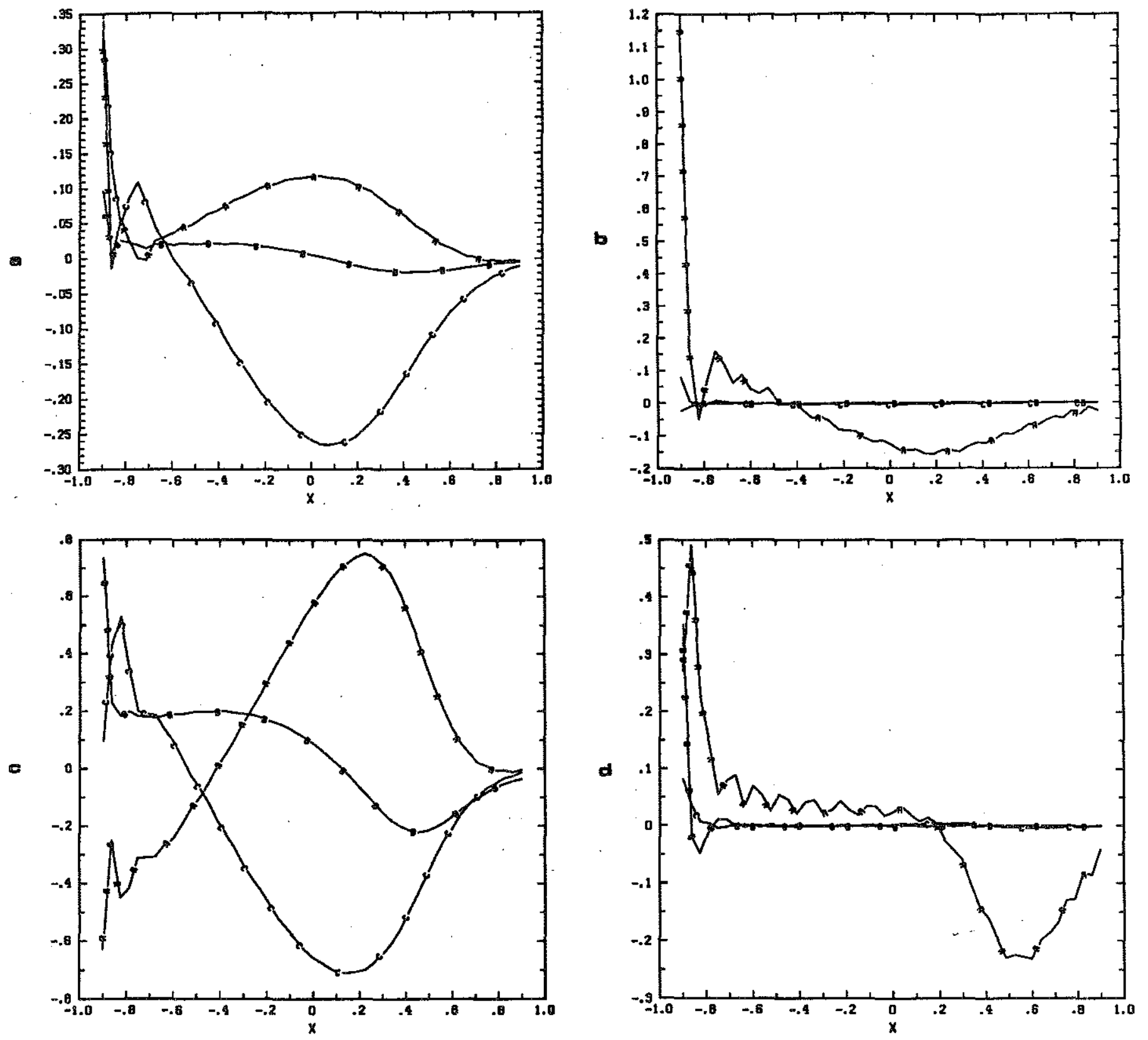

Fig. 3.16. Balance of terms in the lower layer potential vorticity for the experiment C3, as a function of $x$, at fixed latitudes. a) Advective terms at $y=0.85$ : curve $\mathrm{A}=J\left(\psi_{2}, F_{2} \psi_{1}\right)$, curve $\mathrm{B}=J\left(\psi_{2}, y\right)$, curve $\mathrm{C}=J\left(\psi_{2}, h\right)$. b) Viscous and inertial terms at $y=0.85$ : curve $\mathrm{A}=\kappa \nabla^{2} q_{2}$, curve $\mathrm{B}=J\left(\psi_{2}, \gamma \nabla^{2} \psi_{2}\right)$, curve $\mathrm{C}=\delta \nabla^{2} \psi_{2}$. c) Advective terms at $y=0.5$ : curve $\mathrm{A}=J\left(\psi_{2}, F_{2} \psi_{1}\right)$, curve $\mathrm{B}=J\left(\psi_{2}, y\right)$, curve $\mathrm{C}=J\left(\psi_{2}, h\right)$. c) Viscous and inertial terms at $y=0.5$ : curve $\mathrm{A}=\kappa \nabla^{2} q_{2}$, curve $\mathrm{B}=J\left(\psi_{2}, \gamma \nabla^{2} \psi_{2}\right)$, curve $\mathrm{C}=\delta \nabla^{2} \psi_{2}$. 
The numerical experiments, aimed at reproducing the results described in Section 2.2 (constant slope topography), were unsuccessful. In neither the case of wind gyres closing on western boundary layers, nor in the case of wind gyres closing in the interior, did the numerical results agree with the inviscid prediction. Instead, with the latter wind stress pattern, the numerical model gave qualitatively different results from those of the analytic calculation. I have shown that the discrepancy occurs because interfacial friction locks the two layers together making the flow vertically coherent. Therefore the effects that depend crucially on the baroclinicity of the flow are discouraged. This is also why, in the presence of ridge-like topography, the numerical results are in agreement with the inviscid calculation: the jets are present also in a vertically homogeneous model.

The numerical results presented do not rule out the possibility of observing topographically induced baroclinic jets if viscosity is reduced beyond the values I have used. Nevertheless they indicate that the baroclinic jets arising in the presence of constant slope topography are difficult to observe (if they can exist at all) when higher order effects are included. 


\section{Appendix A3 - Description of the numerical model.}

Glenn Ierley's model solves the following quasigeostrophic, two-layer equations (in non dimensional form):

$$
\begin{aligned}
& \frac{\partial q_{1}}{\partial t}+\epsilon J\left(\psi_{1}, q_{1}\right)=g(x, y)+\kappa \nabla^{2} q_{1} \\
& \frac{\partial q_{2}}{\partial t}+\epsilon J\left(\psi_{2}, q_{2}\right)=-\delta \nabla^{2} \psi_{2}+\kappa \nabla^{2} q_{2}
\end{aligned}
$$

where

$$
\begin{aligned}
& q_{1}=\theta_{1}+y=\gamma \nabla^{2} \psi_{1}+F_{1}\left(\psi_{2}-\psi_{1}\right)+y \\
& q_{2}=\theta_{2}+y+h=\gamma \nabla^{2} \psi_{2}+F_{2}\left(\psi_{1}-\psi_{2}\right)+y+h \\
& \epsilon=L_{y} / L_{x} \quad \text { is the aspect ratio of the basin }
\end{aligned}
$$

and

$$
\nabla^{2}=\epsilon^{2} \frac{\partial^{2}}{\partial x^{2}}+\frac{\partial^{2}}{\partial y^{2}}
$$

with boundary conditions $\psi_{1}=\psi_{2}=\theta_{1}=\theta_{2}=0$ on $x= \pm 1, y= \pm 1$.

Solutions are represented in the form:

$$
\left(\begin{array}{c}
\psi(x, y, t) \\
\theta(x, y, t)
\end{array}\right)=\sum_{i=0}^{48} \sum_{j=0}^{48}\left(\begin{array}{l}
\Psi_{i j}(t) \\
\Theta_{i j}(t)
\end{array}\right) T_{i}(x) T_{j}(y)
$$

where $T_{n}(s)$ are the Chebyshev polynomials of the first kind. The $\Theta_{i j}$ are time stepped with a Crank Nicholson scheme, and the $\Psi_{i j}$ are obtained by solving the Poisson and Helmoltz equations resulting from the barotropic and baroclinic streamfunction fields respectively. The diagonalization scheme, introduced by Haidvogel and Zang (1979), is used in the $y$ direction for the Poisson/Helmoltz equations. The remaining problem of 49 coupled linear equations in $x$ is solved reducing the equations to a quasi-tridiagonal form.

The Jacobian terms are calculated in real space, using a NCAR Cray matrix multiplication routine, and use is made of a Cray optimized fast Fourier transform. 
The code contains a diagnostic routine which computes the integral of the diffusive vorticity flux over the whole basin. In the steady state one should find

$$
\begin{aligned}
\kappa \oint \vec{\nabla} q_{1} \cdot \hat{n} d \ell & =-\int d A g(x, y) \\
\kappa \oint \vec{\nabla} q_{2} \cdot \hat{n} d \ell & =\delta \oint \vec{\nabla} \psi_{2} \cdot \hat{n} d \ell
\end{aligned}
$$

with the integrals performed over the whole basin. In all the experiments presented solutions were considered to have reached the steady state when

$$
\begin{aligned}
\kappa^{-1} \frac{\partial}{\partial t} \int d A q_{1} & =\oint \vec{\nabla} q_{1} \cdot \hat{n} d \ell+\int d A g(x, y) / \kappa \approx 10^{-2} \\
\kappa^{-1} \frac{\partial}{\partial t} \int d A q_{2} & =\oint \vec{\nabla} q_{2} \cdot \hat{n} d \ell-\delta / \kappa \oint \vec{\nabla} \psi_{2} \cdot \hat{n} d \ell \approx 10^{-2}
\end{aligned}
$$




\section{References}

De Szoeke R. A., 1985: Wind-driven mid-ocean baroclinic gyres over topography; extension of the Sverdrup relation. J. Mar. Res., 43, 793-824.

Holland W. R., T. Keffer and P. B. Rhines, 1984: Dynamics of the oceanic general circulation: the potential vorticity field.Nature, 308, 698-704.

Ierley G. R. and W. R. Young, 1983: Can the western boundary layer affect the potential vorticity distribution in the Sverdrup interior of a wind gyre?. J. Phys. Oceanogr., 13, 1753-1763.

Luyten J. L., J. Pedlosky and H. Stommel, 1983: The ventilated thermocline. J. Phys. Oceanogr., 13, 292-309.

Luyten J. L., H. Stommel and C. I. Wunsch, 1985: A dignostic study of the Northern Atlantic subpolar gyre. J. Phys. Oceanogr., 15, 1344-1358.

Pedlosky J. and W. R. Young, 1983: Ventilation, potential vorticity homogenization and the structure of the ocean circulation. J. Phys. Oceanogr., 13, 2020-2037.

Rhines P. B. and W. R. Young, 1982a: A theory of wind-driven circulation I. Mid-ocean gyres. J. Mar. Res., 40 (Suppl.), 559-596.

Rhines P. B. and W.R. Young, 1982b: Homogenization of potential vorticity in planetary gyres. J. Fluid Mech., 122, 347-367.

Young W. R. and P. B. Rhines, 1982: A theory of the wind-driven circulation II. Gyres with western boundary layers. J. Mar. Res., 40, 849-872. 


\section{CHAPTER 4}

\section{A model of the inertial recirculation driven by boundary currents: \\ the homogeneous model}

\section{Summary}

Some essential features of the recirculating inertial gyre ("the recirculation") can be analyzed with a simple, analytically tractable model. In wind driven ERGCM the recirculation appears as a strong, sub-basin scale inertial flow with homogeneous potential vorticity. The constant value of potential vorticity decreases with increasing forcing/dissipation ratio while the size and strength of the gyre increase. In the subtropical gyre the recirculation might be driven by anomalous value of low potential vorticity carried northward by the western boundary current. This process is modeled using a barotropic model and prescribing the values of potential vorticity at the edge of the gyre. The gyre is contained in a box in an attempt to simplify the geometry and to isolate the processes occurring in the recirculation region.

With weak diffusion the prescribed boundary forcing induces a flow with constant potential vorticity. The homogenized value of potential vorticity in the interior can be calculated without explicitely solving for the flow. Explicit solutions are also obtained by numerically solving the model. Two distinct cases arise: 1) For strong boundary forcing the gyre fills the box. Therefore the strength of the gyre is determined, but the extent of the recirculation is prescribed. 2) For weak boundary forcing the recirculation fills only part of the box and the size of the gyre can be determined as well as its strength (proportional to the homogenized value of potential vorticity within it). The latter case is the most relevant to the wind-driven, numerical experiments, because in these calculations the recirculating flow is confined to a subbasin-scale region. Also in this case the homogenized value of potential vorticity decreases with increasing forcing, while the size and the strength of the gyre increase. 


\subsection{Introduction}

Since the pioneering work of Fofonoff (1954) on the free inertial circulation in a closed basin, no simple theories have been proposed to understand strongly nonlinear flow.

In Fofonoff's (1954) model, the inertial recirculation fills the whole basin, while observations and results from wind-driven eddy resolving general circulation models show that the nonlinear gyre is confined to a region pressed against the separated boundary current, while in most of the basin, the linear Sverdrup dynamics still dominates (Fig. 4.1).

In the North Atlantic, Schmitz (1980) observed a tight $(\sim 300 \mathrm{~km})$ recirculating gyre, south of the separated Gulf Stream, extending all the way to the bottom with strong eastward and westward velocities $(\sim 5 \mathrm{~cm} / \mathrm{sec})$ with transport of about $90 \mathrm{~Sv}$ at $55^{\circ} \mathrm{W}$. This transport is far too large to be directly driven by the observed wind system through Sverdrup relation, and Worthington (1979) has proposed that buoyancy forcing may be responsible for it.

On the other hand, a gyre with similar features, appears also in a purely winddriven ERGCM pressed against the line of zero wind stress curl (the northern boundary in Fig. 4.1). With the present set of observations it is very hard to tell whether the oceanic recirculation is mainly driven by buoyancy effects or by the wind, therefore, in the following I will take the inertial gyre resulting in wind-driven ERGCM's as a paradigm for comparison with my hypothesis and results. The purpose of this work is to isolate the main dynamical balance and driving mechanism of the recirculation using simple layer models. Before presenting these models, I will summarize the results of some numerical models (eddy resolving and not) which motivate my formulation.

A striking feature appearing in many ERGCM's is that the size and strength of the recirculating gyre depends very weakly on depth. Therefore, to a first approximation, the main features of the recirculation may be captured by a barotropic model. Böning (1986) has reached the same conclusions after a very detailed comparison of ERGCM's 

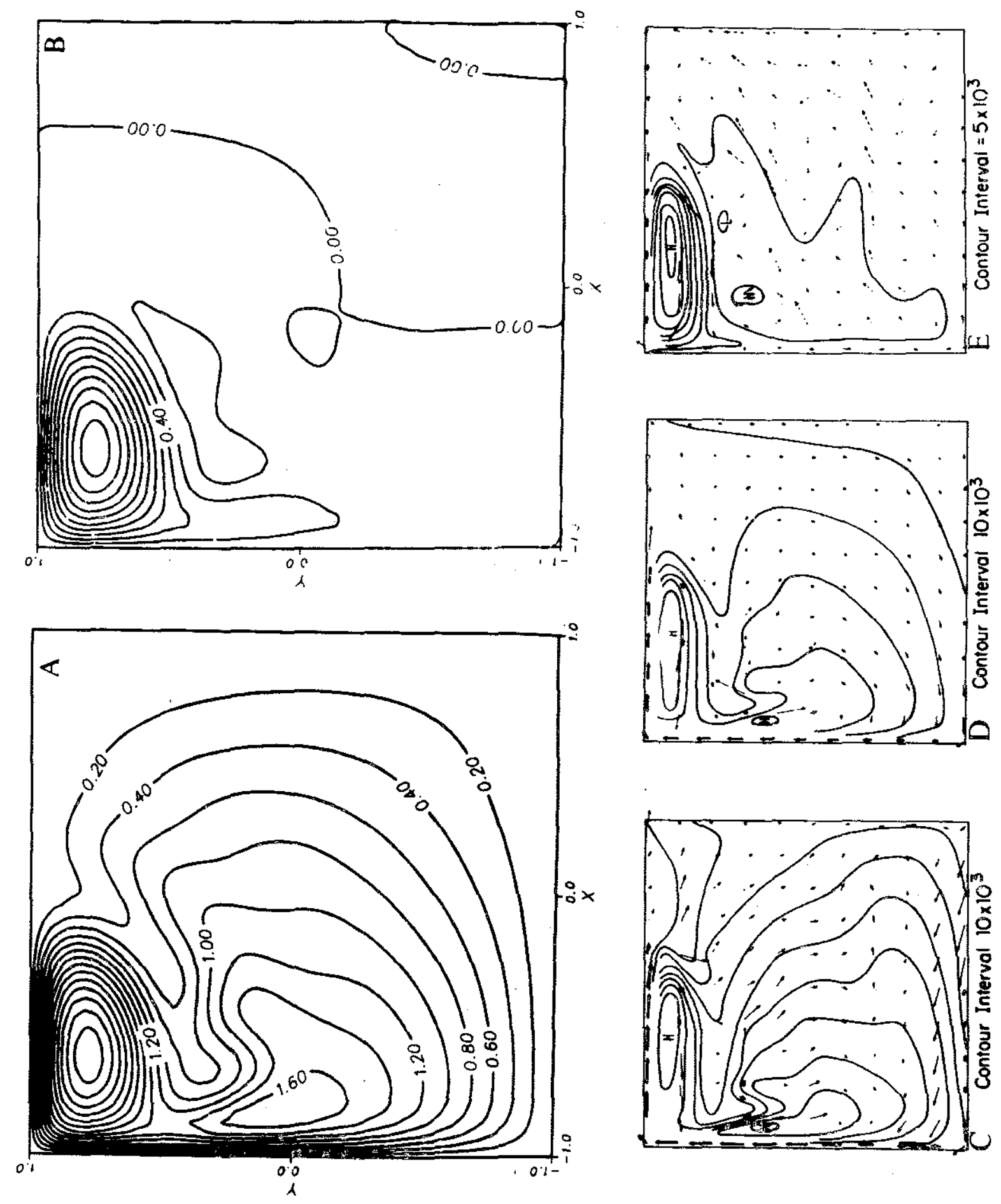

Fig. 4.1. Top row: steady state streamfunction resulting from a two layer quasigeostrophic wind driven GCM. A) Upper layer, B) lower layer. Bottom row: time averaged pressure fields (solid line) and horizontal velocities (arrows) resulting from a 5 level, primitive equation, wind-driven ERGCM (Robinson et al., 1977). (C) fields at $40 \mathrm{~m}$, (D) fields at $490 \mathrm{~m}$, (E) fields at $2690 \mathrm{~m}$. 
results with a simple barotropic numerical model. In the same paper he shows that parameterizing the eddies (not resolved in his barotropic model) with lateral diffusion of mean potential vorticity leads to results in very good agreement with the mean transport fields found in ERGCM's. This is consistent with Rhines and Young's (1982). suggestion that the divergent flux of eddy vorticity can be parameterized as the (negative) gradient of mean potential vorticity.

This evidence justifies the use of a simple model similar to Böning's to analyze in some detail the characteristics of the inertial recirculation.

\section{The Driving Mechanism}

Fig. 4.2 shows the instantaneous streamfunction and potential vorticity field of the final state of a wind-driven barotropic model developed by Dr. Glenn Ierley. The model solves the quasigeostrophic, barotropic equation in a closed basin forced by a simple wind stress curl and damped by lateral diffusion of potential vorticity:

$$
\begin{aligned}
& \frac{\partial q}{\partial t}+J(\psi, q)=\frac{f_{o} w_{e}}{H}+\kappa \nabla^{2} q \\
& \text { where } \quad\left(-\psi_{y}, \psi_{x}\right)=(u, v) \\
& \text { and } \quad q=\beta y+\nabla^{2} \psi \\
& w_{e}=-w_{0} \cos \pi \frac{\left(2 y+L-y_{1}\right)}{2\left(L+y_{1}\right)} \quad \text { if } \quad-L \leq y \leq y_{1} \\
& w_{e}=0 \quad \text { if } \quad y_{1} \leq y \leq L
\end{aligned}
$$

with boundary conditions $\psi=\nabla^{2} \psi=0$ (free slip) on $x= \pm L, y= \pm L$.

The Ekman pumping above was first suggested by Veronis (1966) as a means of illustrating one of the essential features of the recirculation, i.e., it is not locally forced by the wind.

In the experiment shown, the recirculation occurs entirely in a region where the Ekman pumping is zero (north of $y_{1}$ ). The western boundary current continues northward of $y_{1}$, turns eastward at the northern boundary $L$, transporting low values of potential 

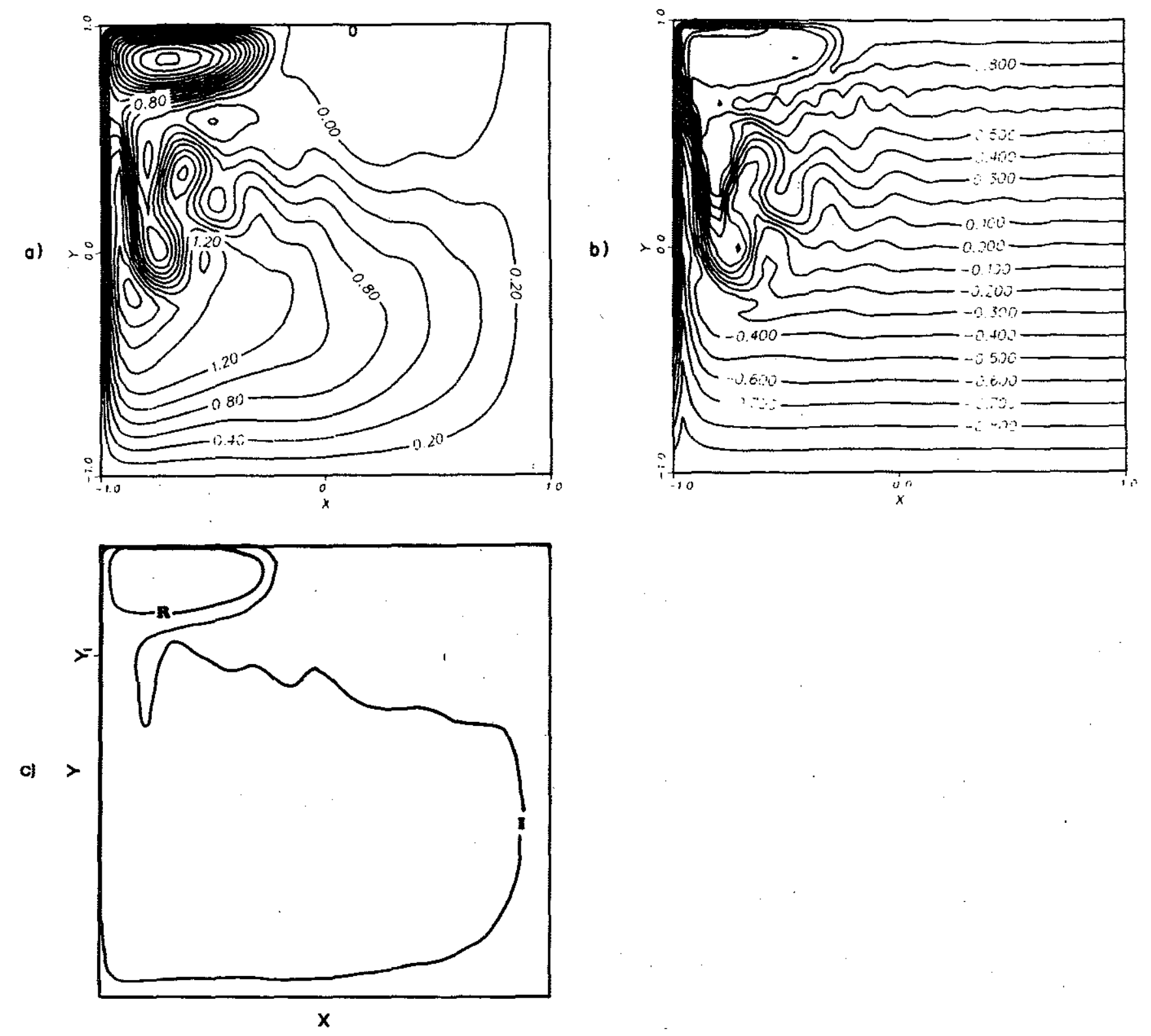

Fig. 4.2. Instantaneous solution at quasi-equilibrium of (4.1.1) with the Ekman pumping (4.1.2). a) Streamfunction, b) potential vorticity. $y_{1}=L / 2, w_{0} f_{0} / H \beta^{2} L^{2}=$ $10^{-3}, \kappa \beta H / w_{0} f_{0} L=2.5 \times 10^{-2} . x$ and $y$ axes in units of $L$. The Ekman pumping is zero in the northern quarter of the basin. c) Schematic picture of two streamlines shown in Fig. 4.2a. The streamline $R$ is in the recirculation region where $w_{e}=0$. The streamline $I$ goes in the interior where $w_{e} \neq 0$. 
vorticity northward, where low means smaller than the local planetary vorticity. The anomalous values of potential vorticity, in turn, drive the strong recirculating gyre. A complementary view is that recirculation occurs to allow particles to move along paths longer than these prescribed by local Sverdrup dynamics, in order to achieve the global balance between forcing and dissipation necessary in the steady state (see Niiler, 1966). A schematic picture of this mechanism is shown in Fig. 4.2c. In the steady state one must have

$$
-\kappa \oint \vec{\nabla} q \cdot d \vec{\ell}=\int d A f_{0} w_{e} / H
$$

where the integrals are performed along any closed streamline.

If the integrals are performed along the streamline $R$ in Fig. 4.2c inside the recirculation, the right hand side is zero and no dissipation occurs inside the recirculation. If the integral is performed along the streamline $I$, which goes in the interior and around the recirculation, the right hand side is almost equal to the integral over the basin of the Ekman pumping (always negative). Comparison with Fig. 4.2b shows that a substantial amount of potential vorticity dissipation occurs near the northwest corner of the basin around the edge of the recirculation gyre, as well as in the western boundary south of the zero wind stress curl line.

The mechanism described above is fairly independent of the choice of the wind stress pattern as suggested in Fig. 4.3, which shows the $\psi$ and $q$ time averaged fields for the same model as. Fig. 4.2, but with a wind stress curl which fills the basin, i.e., $y_{1}=L$ in (4.1.2).

In Fig. 4.4, the potential vorticity field along two streamlines of the recirculation shown in Fig. 4.3 are plotted as a function of arclength $s$. In Fig. 4.4a a streamline at the edge of the recirculation was chosen. Along the western $(2 \leq s \leq 2.5)$ and northern $(0 \leq s \leq 1)$ boundary of the gyre, the potential vorticity (solid line) is much lower than the local planetary vorticity (dashed line) while it is much closer to $\beta y$ elsewhere. 

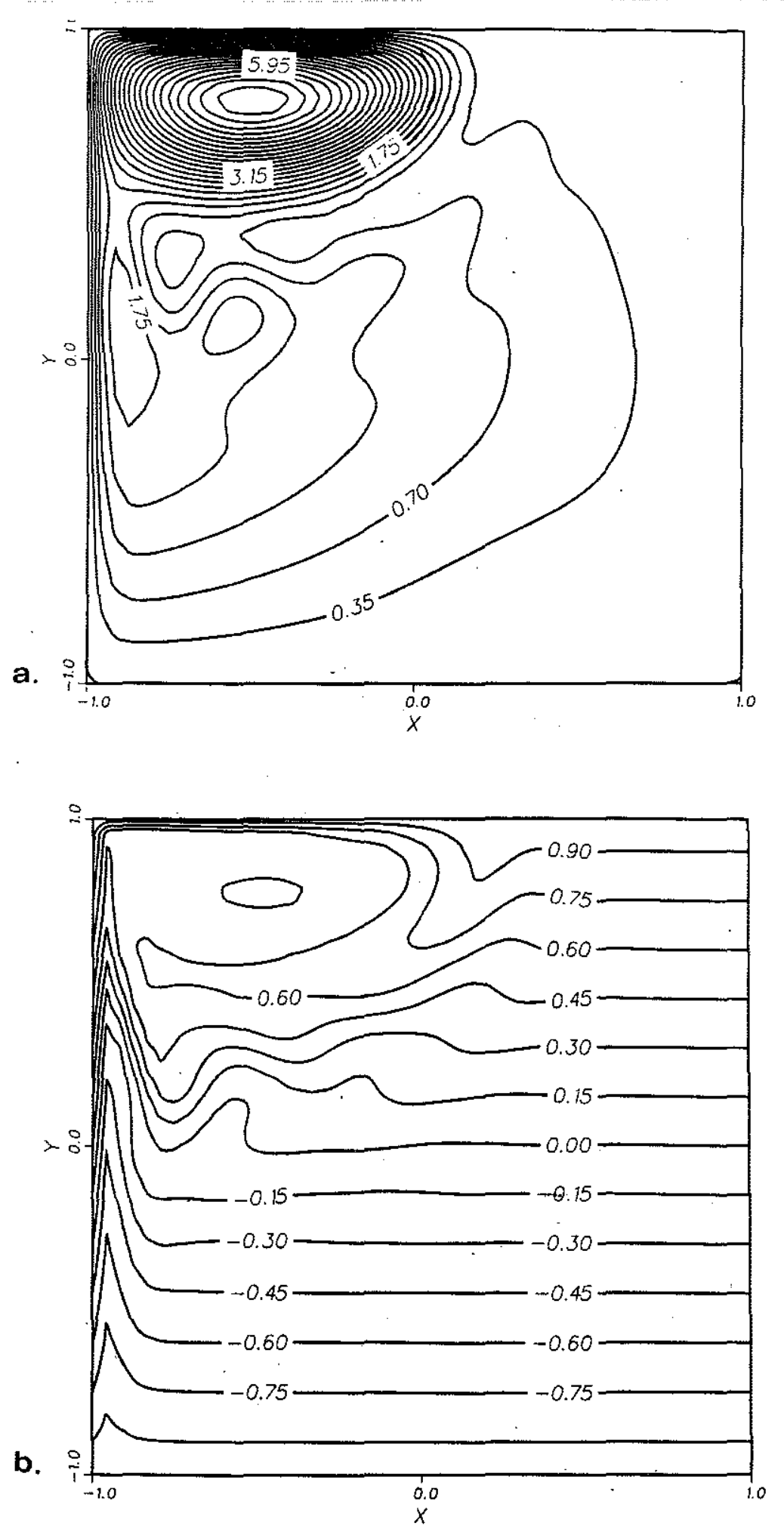

Fig. 4.3. Time averaged fields for the same model as in Fig. 4.2 except for $y_{1}=L$ and $\kappa \beta H / w_{0} f_{0} L=\mathbf{5 . 0} \times \mathbf{1 0}^{-2}$. Now the Ekman pumping is nonzero everywhere. a) Streamfunction. b) Potential vorticity. 
Along the inner streamline (Fig. 4.4b) the variation in potential vorticity is much reduced and indeed potential vorticity. is almost constant.

For smaller values of dissipation/forcing ratio, the recirculating gyre expands and its strength increases. Fig. 4.5 shows the solution when the dissipation is reduced by a factor of two below that in Fig. 4.3. For smaller diffusion, the western boundary current carries even lower values potential vorticity northward. In Fig. 4.6, potential vorticity is plotted as a function of arclength along two streamlines of the recirculation occurring in Fig. 4.5. In Fig. 4.6a a streamline at the edge of the recirculation was chosen. Along the western and northern boundary $(0 \leq s \leq 2.3)$, the difference between potential vorticity (solid line) and planetary vorticity (dashed line) is increased compared to the more viscous case (Fig. 4.4a). This increased forcing is the cause of the more vigorous recirculation. Again along an inner streamline (Fig. 4.6b), potential vorticity is almost constant.

\section{The Dynamical Balance}

The other feature of interest in the recirculation is that potential vorticity is essentially homogeneous (see Figs. 4.3 and 4.5). Because the numerical model is barotropic, the only term that can balance the planetary gradient, $\beta y$, is relative vorticity.

A very detailed diagnostic analysis of general circulation models of the type designed by Dr. Ierley can be found in Böning (1986). An important conclusion of his analysis is that the dominant balance in the recirculation region is between advection of planetary vorticity and advection of relative vorticity. As suggested also by the analysis of the previous section, the wind forcing is negligible inside the recirculation and so is dissipation. Dissipation is very strong around the edge of the recirculation, and indeed is essential in determining the distribution of potential vorticity inside the recirculation. 

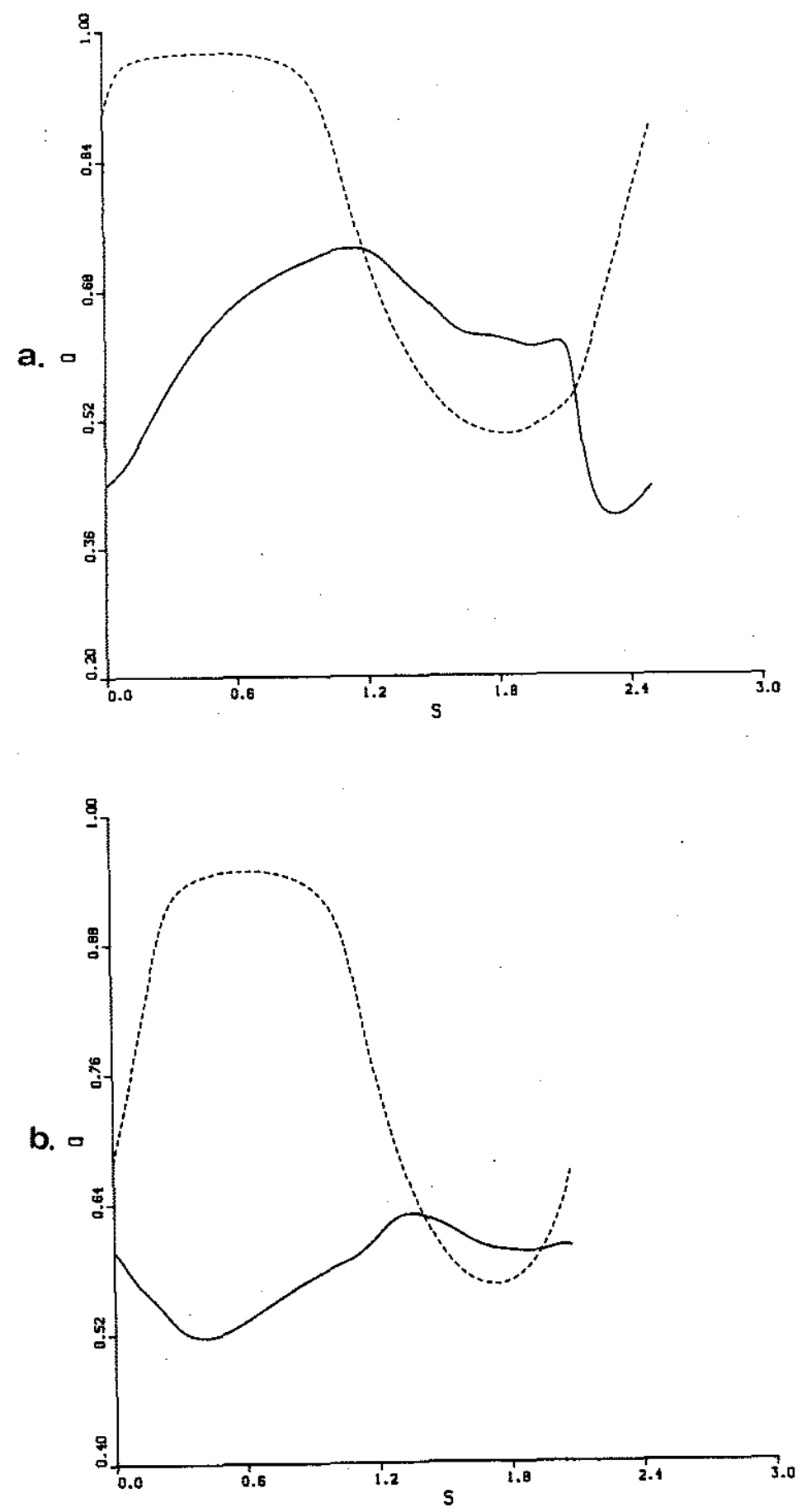

Fig. 4.4. Total potential vorticity $q$ (solid lines) and planetary vorticity $\beta y$ (dashed lines) as a function of arclength (moving clockwise) along streamlines in the recirculation region of Fig. 4.3. a) $q$ and $\beta y$ along a streamline at the edge of the gyre, $\psi=2.0$. b) $q$ and $\beta y$ along a streamline in the interior of the gyre, $\psi=3.5$. Notice the change in the ordinate scale relative to Fig. 4.4a. 
a)

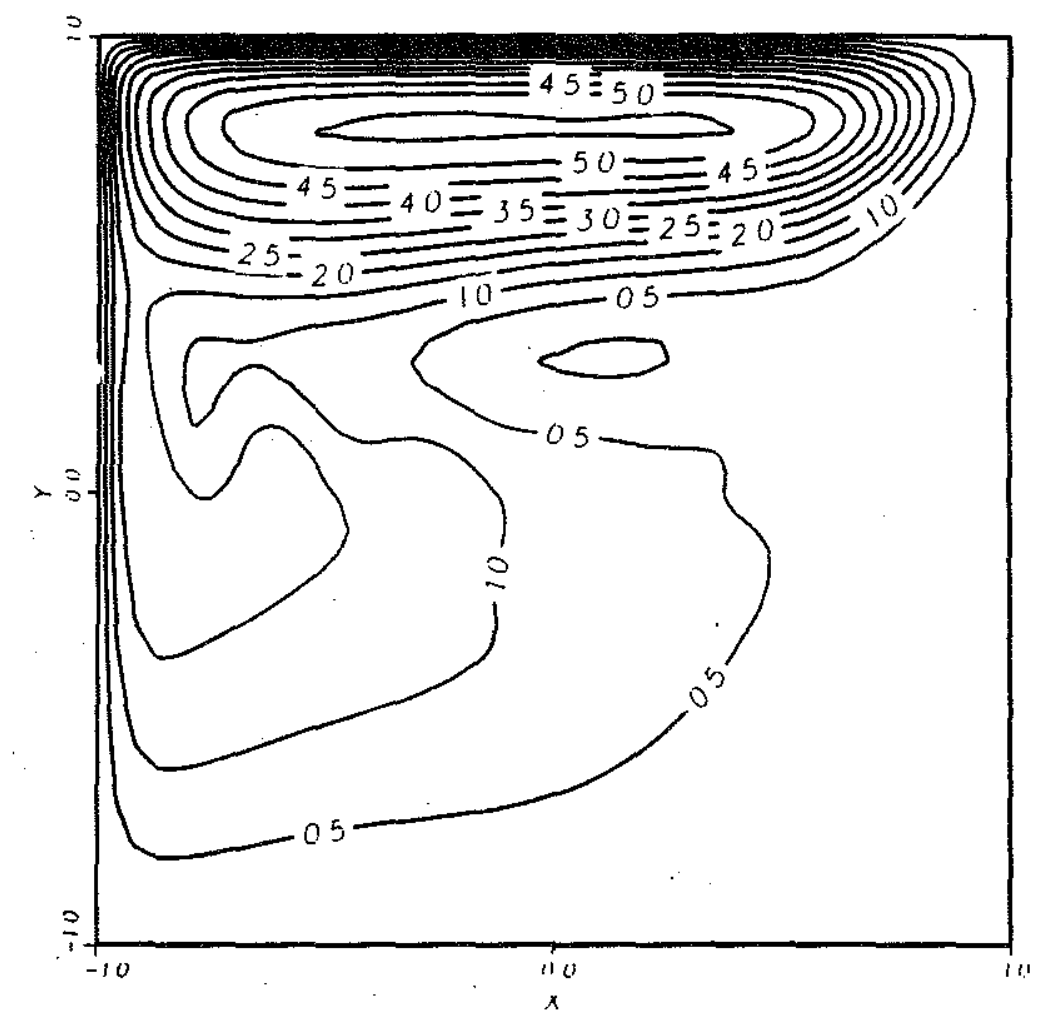

b)

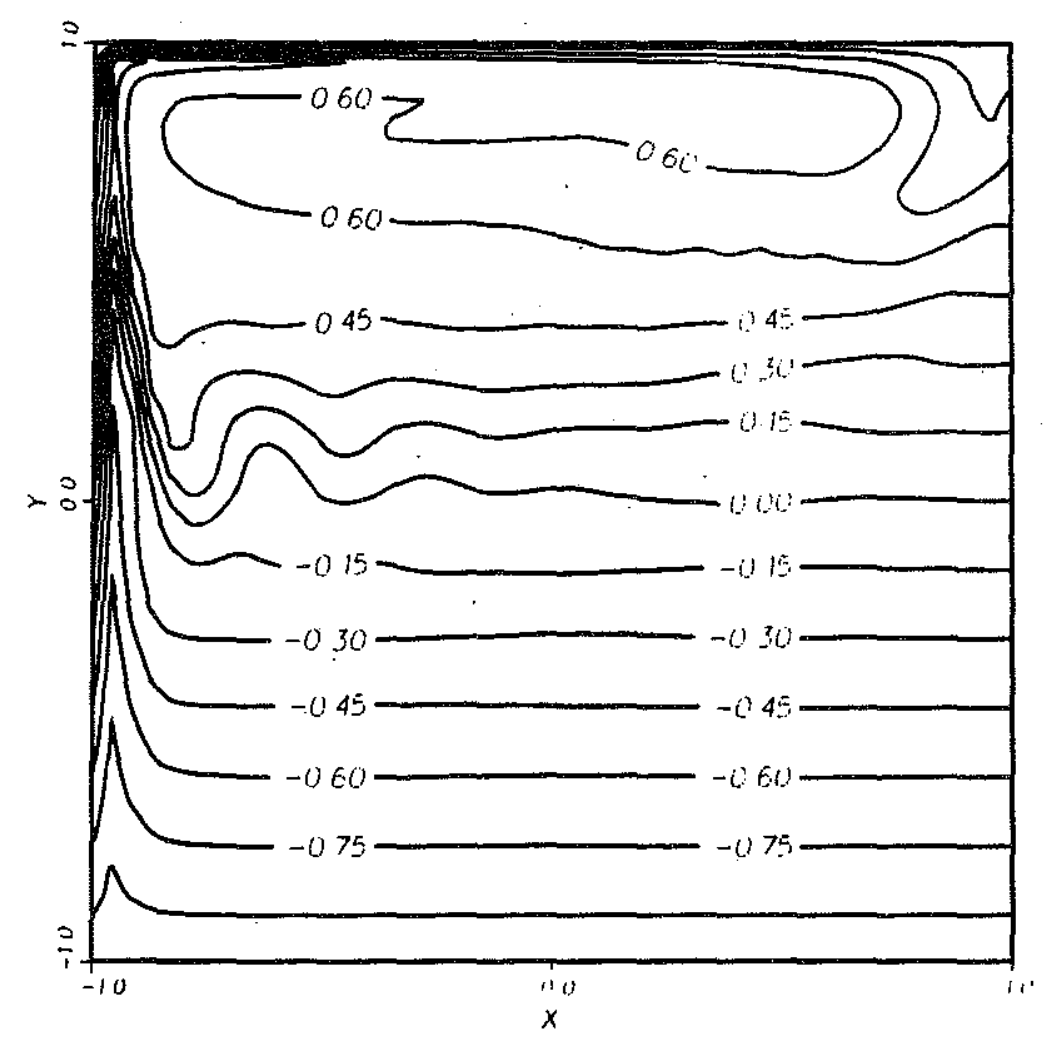

Fig. 4.5. Same as Fig. 4.3 except for $\kappa \beta H / w_{0} f_{0} L=2.5 \times 10^{-2}$. The recirculation has expanded relative to that in Fig. 4.3 because diffusivity has been halved. 

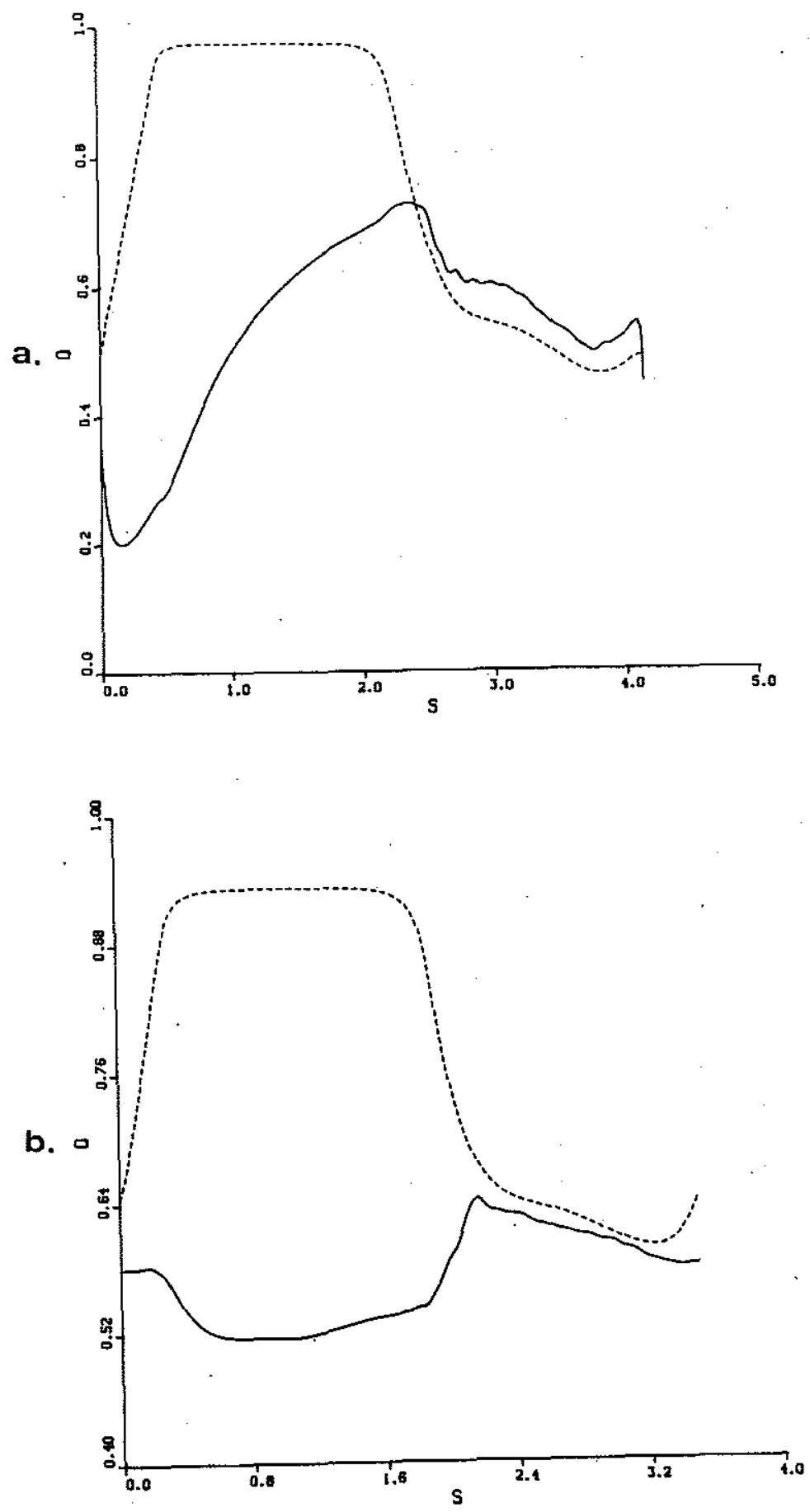

Fig. 4.6. Total potential vorticity $q$ (solid lines) and planetary vorticity $\beta y$ (dashed lines) as a function of arclength (moving clockwise) along streamlines in the recirculation region of Fig. 4.5. a) $q$ and $\beta y$ along a streamline at the edge of the gyre, $\psi=2.5$. The difference between $q$ and $\beta y$ is increased relative to the more viscous case shown Fig. 4.4a.

b) $q$ and $\beta y$ along a streamline in the interior of the gyre, $\psi=3.5$. Note the change in the ordinate scale relative to Fig. 4.6a. 
Relative vorticity is important also in the westward flow of the recirculation, and this is very different from the picture envisaged by Fofonoff (1954) where relative vorticity is confined to narrow boundary layers at the northern, western and eastern edges of the gyre. Therefore, a correct model of the recirculation must include relative vorticity in the interior region of the inertial gyre.

One final observation from numerical experiments is that the tangential velocity at the boundary of the recirculation is essentially determined by the dynamics of the interior region. In Fig. 4.7, the zonal velocity, $u$, and the potential vorticity, $q$, are shown for a section that cuts the recirculation region longitudinally ( $x=0$ in Fig. 4.5). No jump in $u$ occurs when the northern boundary is approached. Clearly there is a jump in $q$, in order to satisfy the free-slip boundary condition, causing $q_{y}$ to be large near the boundary. But $q$, although discontinuous, is finite, and the velocity on the boundary can be predicted approximately by extrapolating the interior value from just outside the boundary layer.

\section{Summary}

Recirculation is the development of a quasi-barotropic, sub-basin scale gyre, confined to the northwest corner of the subtropical recirculation, forced by anomalous values of potential vorticity at the edge of the gyre.

The transport of this gyre is much larger than the Sverdrup transport. Everywhere in the gyre, relative vorticity is as important as planetary vorticity. Although the dissipative terms and the wind forcing are subdominant, their strength sets the amplitude and the size of the recirculating gyre.

There are several questions suggested by these observations. What determines the latitudinal extent of the gyre? What determines the value of potential vorticity in the center of the gyre? Why is the recirculation almost barotropic?

In this chapter, the first two questions are addressed using a barotropic model. In the next chapter, the third question is answered using a two-layer model. 

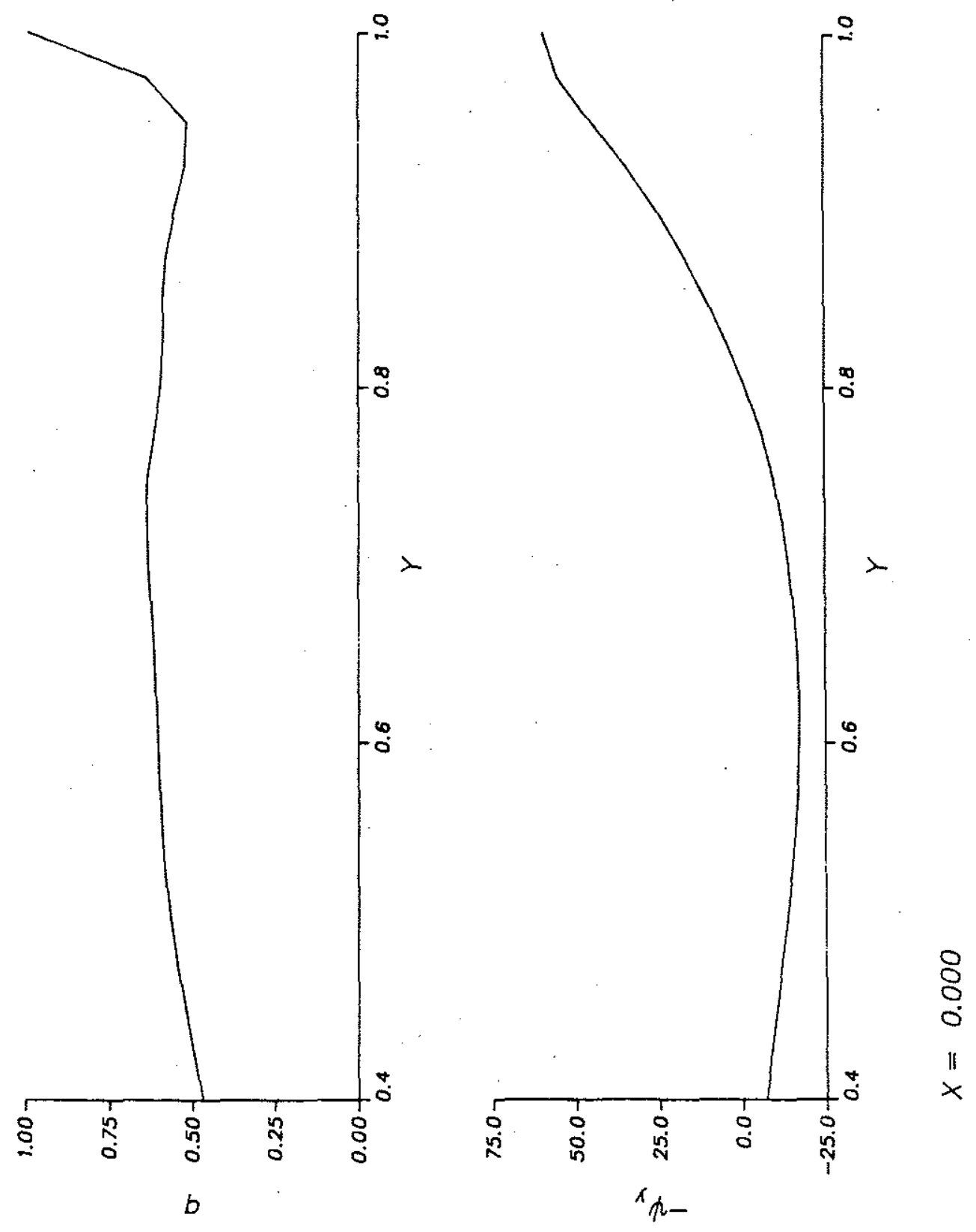

Fig. 4.7. a) Zonal velocity $u$ and potential vorticity $q$ through the recirculation region as a function of $y$, at $x=0$. There is no jump in velocity near the northern boundary, but there is a jump in potential vorticity. All parameters are as in Fig. 4.5. 


\subsection{Formulation of the Barotropic Model}

In order to highlight the essential features of the recirculation, I will analyze a model which is as simple as possible. Therefore, the following assumptions will be made:

1. The inertial recirculation is barotropic. This constraint will be removed later and results for the baroclinic model will justify this choice a posteriori.

2. Relative vorticity is of the same order as planetary vorticity.

3. Diffusion is weak and can be represented as lateral diffusion of potential vorticity.

4. There is no local wind or buoyancy forcing. Instead, the forcing is provided by prescribing the potential vorticity at the boundary of the recirculation (this idea is due to $\mathrm{W}$. Young and myself). This mimics the effect of the western boundary current carrying low values of potential vorticity northward as observed in Figs. 4.4 and 4.6, or can be thought of as some thermally induced potential vorticity anomaly concentrated at the boundary current.

5. The recirculation is considered isolated from the Sverdrup interior, which, together with the wind forcing, is neglected.

With the above in mind, we consider the quasigeostrophic equation in a $\beta$-plane box $(-L / \epsilon \leq x \leq L / \epsilon$ and $-L \leq y \leq L)$

$$
\frac{\partial q}{\partial t}+J(\psi, q)=\kappa \nabla^{2} q
$$

where $q=\nabla^{2} \psi+\beta y$ and $\epsilon$ is the aspect ratio of the box. The boundary conditions are $\psi=0$ and $q=q_{b}(s)$, where $s$ is the arclength around the box. Thus the flow is forced by the distribution of relative vorticity prescribed at the boundary $\left(q_{b} \neq \beta y\right)$.

The eastern and western boundaries represent the actual eastern and western boundaries of the basin, while the northern and southern boundaries are the meridional boundaries of the recirculation. Implicitly, a situation such as that depicted in Fig. 4.5 is considered, where the forcing/dissipation is strong enough so that the recirculation goes all the way to the eastern boundary. 
Before discussing specific functional forms for $q_{b}(s)$, some general results relevant to this problem can be proved.

Integrating (4.2.1) over the area enclosed by any streamline, and assuming a steady solution, we get

$$
\kappa \oint \vec{\nabla} q \cdot \hat{n} d \ell=0
$$

where $\hat{n}=\vec{\nabla} \psi /|\vec{\nabla} \psi|$. That is, the total diffusive flux across a closed streamline is zero.

Multiplying (4.2.1) by $\psi$ and integrating over the area enclosed by the box, we obtain the energy equation

$$
\frac{\partial}{\partial t} \int \frac{|\vec{\nabla} \psi|^{2}}{2} d A=-\kappa \int d A\left(\nabla^{2} \psi\right)^{2}+\kappa \oint\left(q_{b}-\beta y\right) \vec{u}_{b} \cdot \overrightarrow{d \ell}
$$

In the steady state the amount of vorticity available in the interior is proportional to the relative vorticity circulated at the boundaries. If, as a trivial example, $q_{b}=$ $\beta y$ then the relative vorticity must be identically zero everywhere in the interior and therefore there is no flow. This emphasizes the role of $q_{b}$ as forcing.

Equation (4.2.1) is analogous to an advection diffusion equation for the concentration of a passive tracer with prescribed values at the boundaries. It is intuitively clear that the concentration in the interior must have (in the steady state) values lying between the boundary values. This result can be proved rigorously even for potential vorticity, which is not a passive scalar, since the proof does not make use of the definition of $q$. This result was proved by Dr. Young and will be repeated here.

Suppose there is a point in the interior where $q$ is larger (smaller) than any of the values applied at the boundaries. In this case, such a point is a maximum (minimum). Therefore, around this point there will be a nested set of closed $q$ contours. Integrating (4.2.1) over the area enclosed by any such contour and assuming a steady state, we find

$$
\text { where } \begin{aligned}
\kappa \oint \vec{\nabla} q \cdot \hat{n} d \ell & =\kappa \oint|\vec{\nabla} q| d \ell=0 \\
\hat{n} & =\vec{\nabla} q /|\vec{\nabla} q|
\end{aligned}
$$


which is a contradiction. Therefore in the steady state there cannot be closed $q$ contours. A very important corollary of this result is that there cannot be any point where a discontinuity in velocity is smoothed over a distance which decreases as diffusion is reduced. If a shear layer occurred, then relative vorticity $\nabla^{2} \psi$ would become very large and $q$ would exceed the boundary values. This result is not in contradiction with the statements made in chapters 2 and 3 about the geometry of the potential vorticity contours. For example in Fig. 2.3 the quantity that is contoured is $q_{2}$ assuming that the lower layer is at rest. Indeed it is to avoid closed potential vorticity contours that the lower layer must move inside the circle $r=r_{1}$ shown in Fig. 2.3, keeping $q_{2}$ constant. In the presence of weak, but nonzero, viscosity (4.2.4) requires that the potential vorticity contours spiral around the region of homogenized $q$ as depicted in Fig. 3.11, so that a closed potential vorticity contour never occurs.

In proving these general results, no assumption about the size of diffusion has been made. In order to make further progress, the weakly diffusive limit will be considered, limit which is probably the most relevant for the oceanic recirculation. 


\subsection{Homogenized Gyre Filling the Box}

With the simplest possible choice of $q_{b}$, Dr. Glenn Ierley has solved (4.2.1) numerically. Specifically, the form for $q_{b}$ chosen is:

$$
q_{b}=\left(Q_{n}-Q_{\theta}\right) \frac{(y-L)}{2 L}+Q_{n}
$$

where $Q_{n}$ and $Q_{s}$ are the constant values of $q$ on the northern and southern boundaries, respectively. Typical steady state solutions are shown in Figs. 4.8, 4.9, and 4.10. For some values of $Q_{n}$ and $Q_{s}$, the recirculating gyre fills the whole box, while for other choices it is confined to a subregion of the basin. In this section, I will examine cases where the circulation fills the whole domain as in Fig. 4.8. The more complicated and interesting case typified by Fig. 4.10 will be analyzed in the next section.

In the limit of weak diffusion, outside the thin boundary layers close to the solid walls, potential vorticity is homogeneous. This is a consequence of (4.2.2) in the limit of small dissipation (see also Rhines and Young, 1982). But what is the constant value of potential vorticity inside the recirculation region?

The question is answered in Appendix A4 and the result is that the constant value of potential vorticity in the interior is given by

$$
\vec{q}=\frac{\oint q_{b} \vec{u} \cdot d \vec{\ell}}{\oint \vec{u} \cdot d \vec{\ell}}
$$

where the integral is performed along the boundary of the domain. The detailed derivation is given in Appendix A4, but the physical explanation is very simple (Roberts, 1977). As the fluid is advected along the streamlines, potential vorticity is diffused from streamline to streamline, since streamwise diffusion is always negligible with respect to advection. Cross-stream diffusion is more effective when streamlines are closer, that is where the velocities are larger. This is why, in the determination of $\bar{q}$, the boundary values of $q$ contribute more where velocities are larger. Therefore, this velocity weighted average is opposite to a time average which would be weighted with the inverse of the 

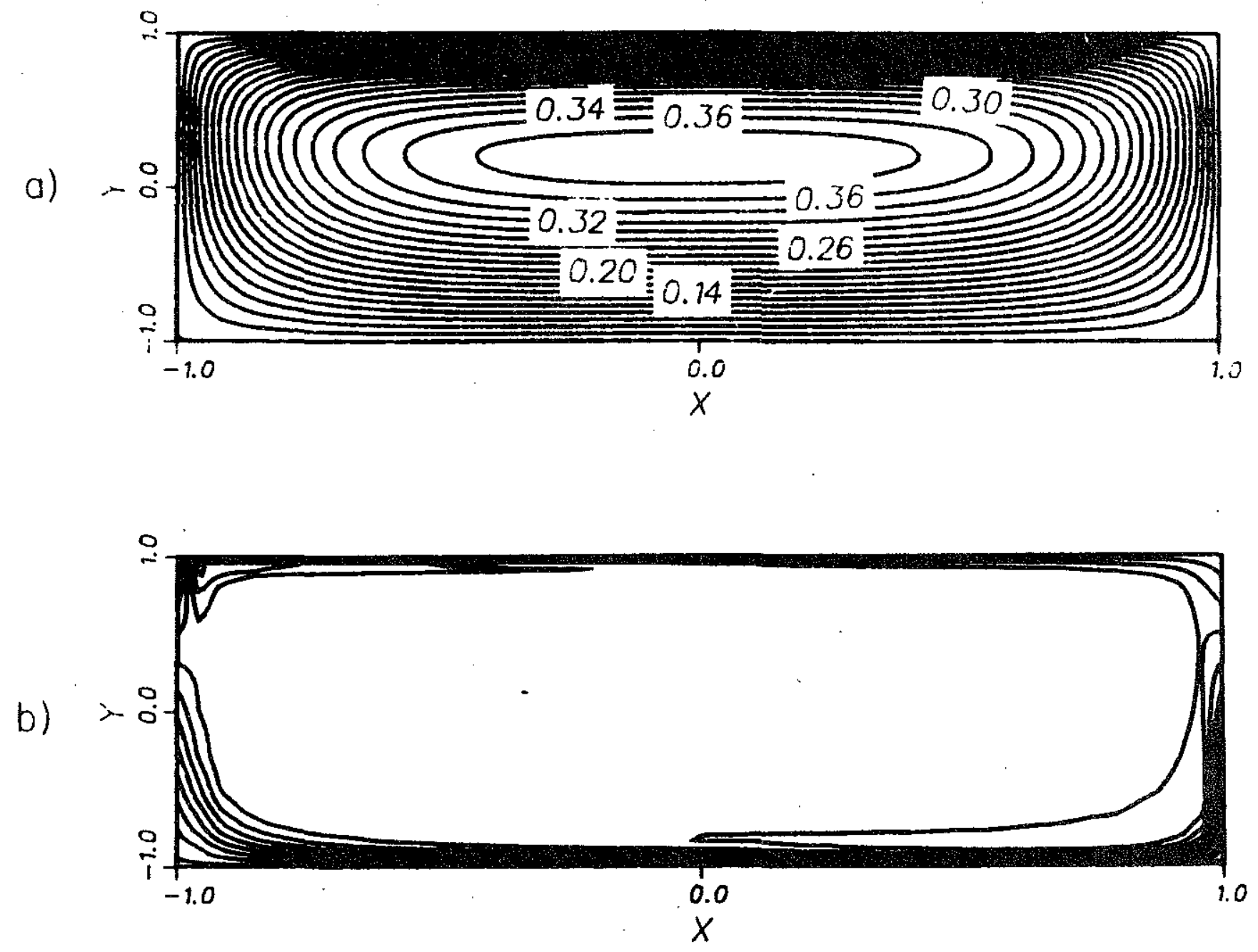

Fig. 4.8. a) Streamfunction and b) potential vorticity for the steady state resulting from the numerical solution of (4.2.1) with potential vorticity boundary condition (4.3.1). $Q_{n}=-2 \beta L / 3, Q_{s}=-\beta L . \epsilon=0.3, \kappa / \beta L^{3}=5 \times 10^{-4}$. Streamfunction is in units of $\beta L^{3}$ and potential vorticity is in units of $\beta L$. x-axis in units of $L / \epsilon, y$-axis in units of $L$. Potential vorticity contour interval is 0.03 . Notice the presence of small closed $q$ contours, due to a residual time dependence. 
a)
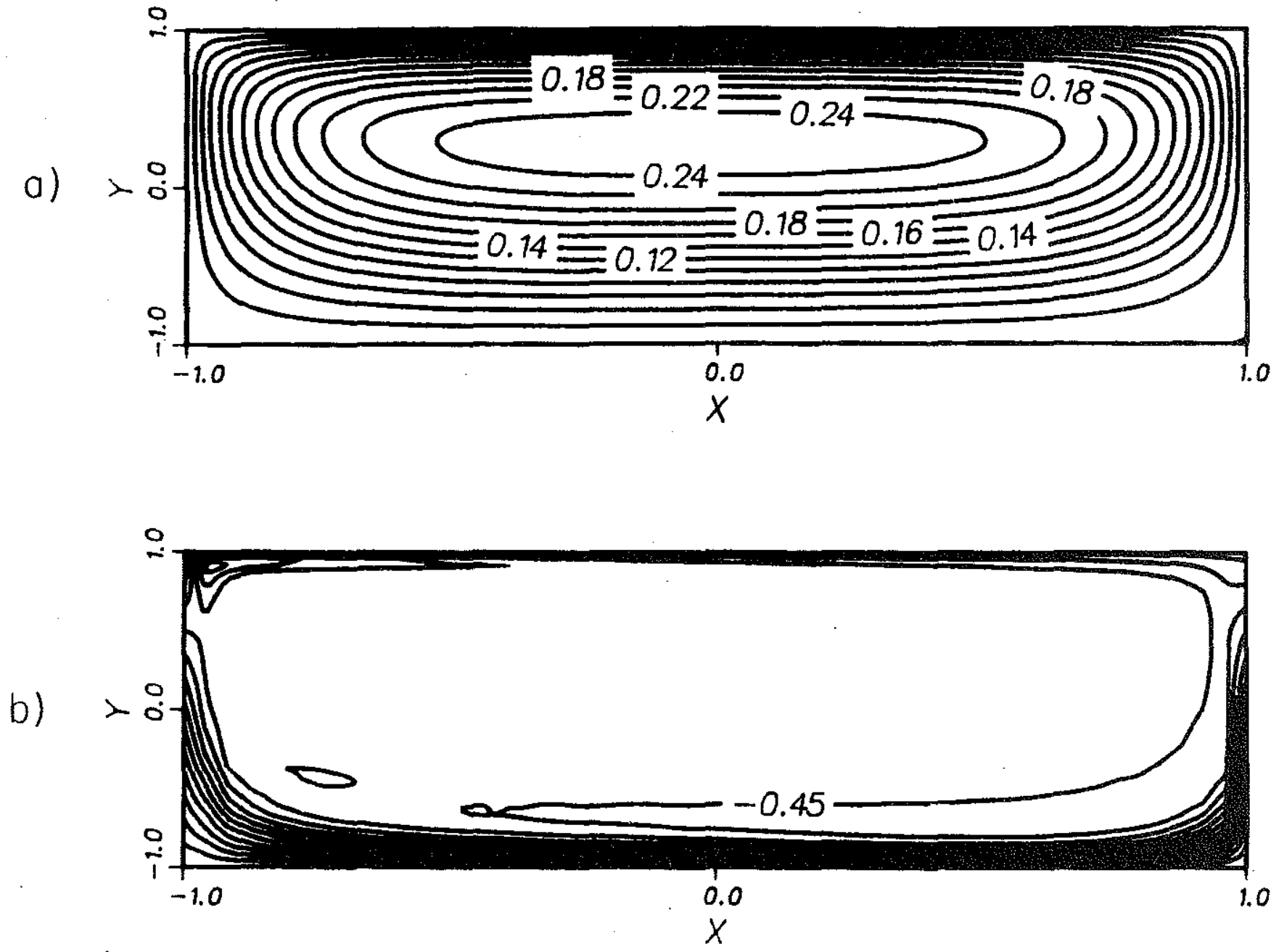

Fig. 4.9. a) Streamfunction and b) potential vorticity for the steady state resulting from the numerical solution of (4.2.1) with potential vorticity boundary condition (4.3.1). $Q_{n}=-\beta L / 3, Q_{s}=-\beta L . \epsilon=0.3, \kappa / \beta L^{3}=5 \times 10^{-4}$. Streamfunction is in units of $\beta L^{3}$ and potential vorticity is in units of $\beta L . x$-axis in units of $L / \epsilon, y$-axis in units of $L$. Potential vorticity contour interval is 0.05 . 
a)

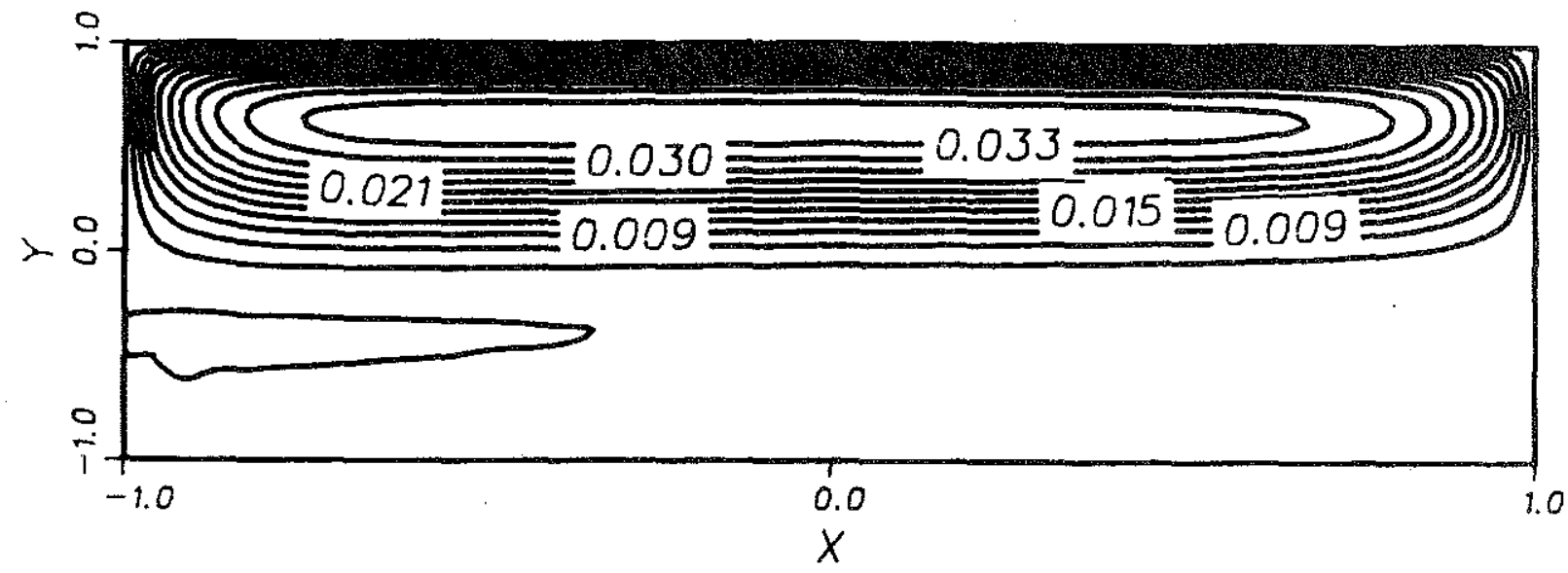

t)

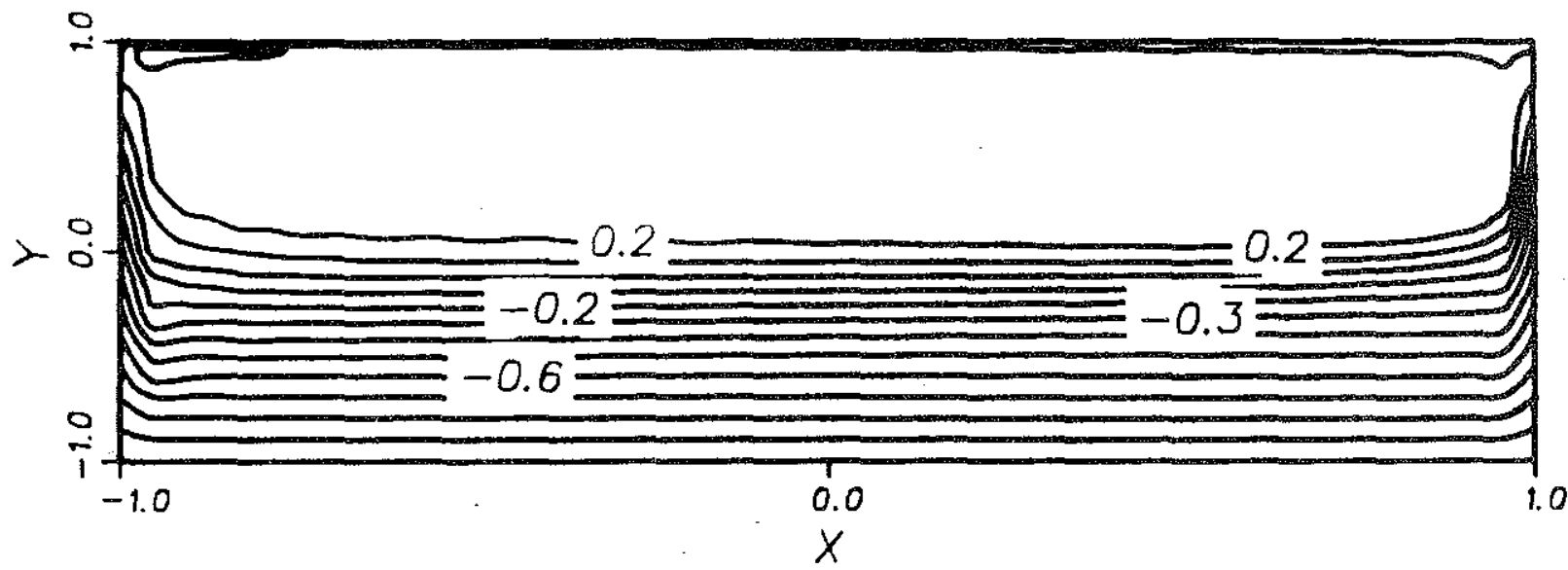

Fig. 4.10. a) Streamfunction and b) potential vorticity for the steady state resulting from the numerical solution of $(\mathbf{4 . 2 . 1})$ with potential vorticity boundary condition (4.3.1). $Q_{n}=\beta L / 3, Q_{a}=-\beta L . \epsilon=0.3, \kappa / \beta L^{3}=6.75 \times 10^{-4}$. Streamfunction is in units of $\beta L^{3}$ and potential vorticity is in units of $\beta L . x$-axis in units of $L / \epsilon, y$-axis in units of $L$. Potential vorticity contour interval is 0.1 . 


\begin{tabular}{|c|c|c|c|c|}
\hline$\kappa^{\prime}$ & $\psi^{\prime}$ & $q_{\min }^{\prime}$ & $q_{\max }^{\prime}$ & $\bar{q}^{\prime}$ \\
\hline 0.050 & 2.0 & 0.395 & 0.733 & 0.603 \\
0.050 & 3.5 & 0.517 & 0.706 & 0.567 \\
0.050 & 5.0 & 0.523 & 0.547 & 0.535 \\
\hline 0.025 & 1.75 & 0.200 & 0.724 & 0.527 \\
0.025 & 2.50 & 0.402 & 0.664 & 0.531 \\
0.025 & 3.50 & 0.514 & 0.639 & 0.553 \\
\hline
\end{tabular}

Table 4.1: Potential vorticity along streamlines in the recirculating gyres shown in Figs. 4.3 and 4.5. The last column is the "velocity weighted" average obtained from Eq. 4.3.2. The upper three rows are from the experiment shown in Fig. 4.3. The lower three rows are from the experiment shown in Fig. 4.5 where viscosity has been halved. Notice that although the range of potential vorticity values is larger the "average" value varies less from streamline to streamline. The prime indicates that the quantities are nondimensional.

velocity. Equation 4.3.2 is valid also if the integrals are performed along any closed streamline. I have computed the "velocity weighted" average for the wind-driven experiments shown in Figs. 4.3 and 4.5 for three sets of streamlines. The first set (lowest value of $\psi$ ) is just at the boundary of the recirculation and the second two sets (higher value of $\psi$ ) are well inside the recirculation where $q$ is almost homogeneous. In each experiment, the average value of $q$ should be the same for any streamline. The results are shown in Table 4.1 together with the maximum and minimum value of potential vorticity encountered on the streamline. Notice that the difference in the average value of $q$ from streamline to streamline is smaller in the less viscous experiment although the range of potential vorticity value on the streamline is wider.

As was noted in the Introduction and illustrated in Fig. 4.7 for the wind-driven experiment, the velocity at the boundary is essentially determined by the interior dynamics where potential vorticity is constant. In this model, this is assured by the extremum principle which excludes shear layers. In the interior region potential vorticity is uniform and the flow satisfies

$$
\nabla^{2} \psi+\beta y=\bar{q}
$$


with boundary conditions $\psi=0$ on $x= \pm L / \epsilon, y= \pm L$. Using (4.3.3) and the fact that $q_{b}$ is a linear function of $y$, the expression for $\bar{q}$ can be put in the form (see Appendix B4 for a more detailed derivation)

$$
\bar{q}=\frac{\int d A q_{b}(\bar{q}-\beta y)}{\int d A(\bar{q}-\beta y)}
$$

where $q_{b}$ is now defined everywhere in the interior and is given by (4.3.1). This result can be generalized to any form of $q_{b}(s)$ and the derivation is given in Appendix B4. Evaluating the trivial integrals in (4.3.4), a simple quadratic equation for $\bar{q}$ is obtained with solutions

$$
\bar{q}=\frac{Q_{n}+Q_{s}}{4} \pm \frac{1}{4} \sqrt{\left(Q_{n}+Q_{s}\right)^{2}-8 \beta L\left(Q_{n}-Q_{s}\right) / 3}
$$

Notice that the aspect ratio $\epsilon$ does not enter in this formula. In deriving (4.3.5), we have made the following assumptions about the solution of (4.3.3):

(a) The gyre fills the whole domain.

(b) There is only one gyre.

Both these assumptions must be checked $a$ posteriori by solving (4.3.3) with the value of $\bar{q}$ obtained from (4.3.5). Also, unphysical roots, such as complex values and values that are outside the range of the boundary values, must be eliminated. The solutions in (4.3.5) actually depend only on the two non-dimensional parameters $n=Q_{n} /(\beta L)$ and $s=Q_{s} /(\beta L)$, and the parabola bounding the domain of real solutions in the $n-s$ plane shown in Fig. 4.11. Notice the symmetry around the axis $n=-s$. Changing $n+s$ into $-(n+s)$ with $n-s$ constant just reverses the sign of $\bar{q}$. Therefore, I will only analyze the half-plane $n+s \leq 0$.

As mentioned earlier, the homogenized value of $q$ given by (4.3.5) must be consistent with the hypothesis made to derive this result. In other words, the solution of (4.3.2) must consist of only one gyre filling the whole box. The geometry of the box (which does not enter (4.3.5) as noted earlier) restricts the allowable values of $n$ and $s$. 


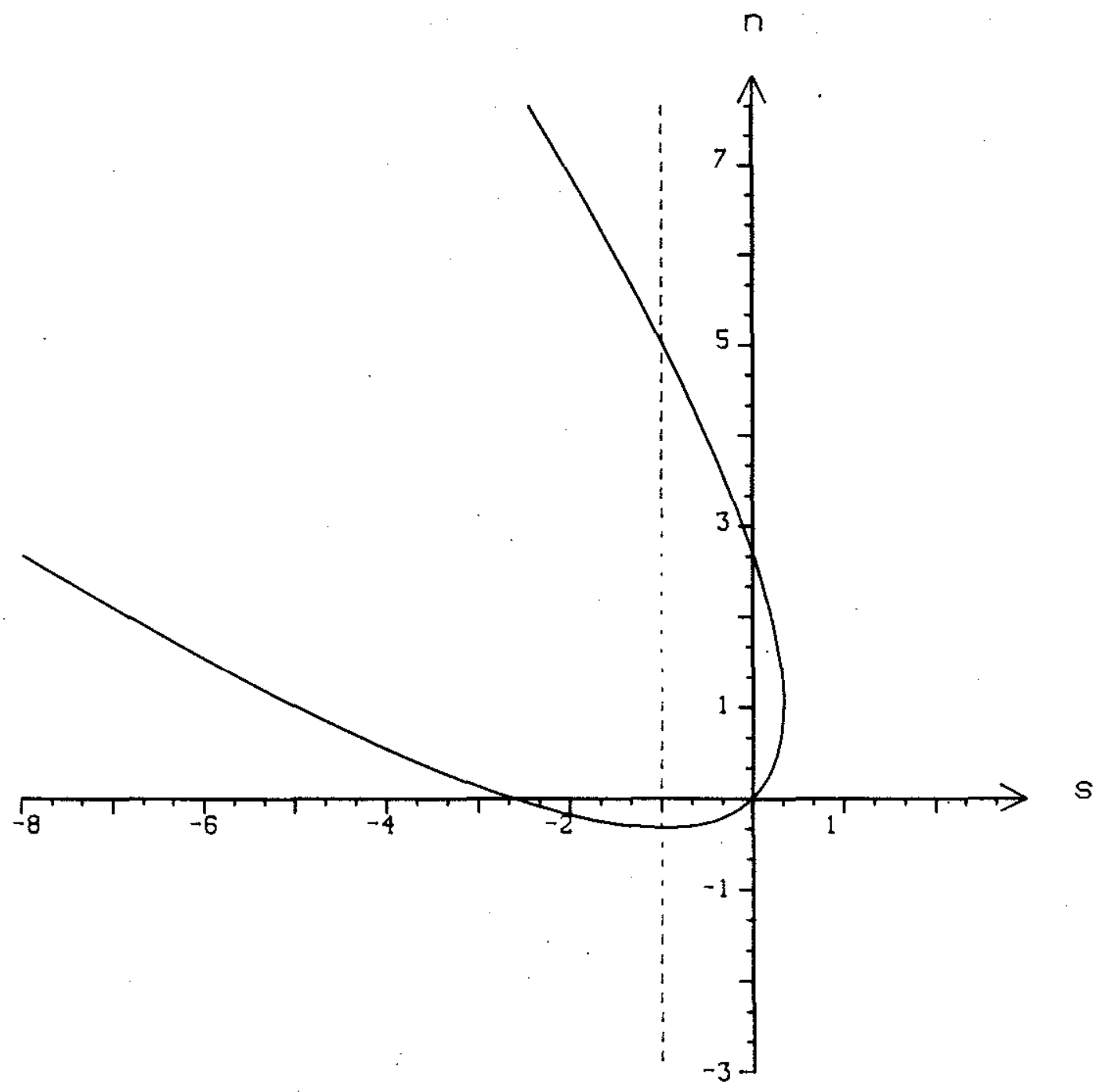

Fig. 4.11. Parabola bounding the domain of real solutions of (4.3.5) as a function of $n=Q_{n} / \beta L$ and $s=Q_{a} / \beta L$. Inside the parabola (4.3.5) has complex roots. The dashed line is at $s=-1$. 
To illustrate this point I have considered a different geometry where analytical solutions of (4.3.3) can be found, i.e., an elliptical domain. The discussion of this problem is deferred to Appendix C4. In the following, using physical arguments, I will restrict my attention to a certain portion of the parameter space $\left(Q_{a}=-\beta L, Q_{n}<\beta L, \epsilon^{2} \ll 1\right)$ which is most relevant to the recirculation.

In the wind-driven models, the main source of vorticity for the recirculation is the western boundary current. As the flow circulates along the streamlines surrounding the recirculation, it carries low values of potential vorticity northward (i.e., $Q_{n}<\beta L$ ) while dissipation acts to bring potential vorticity back to its local planetary value. Because the circulation is clockwise, when the flow has reached the southern edge of the recirculation, dissipation has eroded the relative vorticity injected at the boundary (see Fig. 4.6). No source of relative vorticity is present at the southern edge where the recirculation is bounded by the Sverdrup interior where potential vorticity is essentially given by its planetary value. Therefore, I have concentrated my attention on the case where $Q_{s}=-\beta L(s=-1$ in Fig. 4.11). For the numerical experiment shown in Fig. 4.8, $Q_{a}=-\beta L$ and $Q_{n}=-2 / 3 \beta L$. For this choice, (4.3.5) gives two roots: $\bar{q}=-0.76 \beta L$ and $\bar{q}=-0.073 \beta L$. The latter is clearly unphysical because it gives a value for the homogenized $\bar{q}$ which is outside the boundary value. The solution obtained by Dr. Ierley integrating (4.2.1) numerically gives a value for $\bar{q} \sim-0.77 \beta L$ in excellent agreement with the theoretical prediction. 


\subsection{Homogenized Gyres with Free Boundaries}

The case which is most relevant to the wind-driven circulation is when the forcing provided by the potential vorticity boundary condition is weak. That is, when the values of $Q_{n}$ and $Q_{s}$ are chosen such that (4.3.5) does not have real roots. The reason why this region of the parameter space is called "weak forcing" is because it contains the point $n=1, s=-1$ (see Fig. 4.11). This corresponds to the choice $q_{b}=\beta y$ for the boundary forcing. As shown by (4.2.3) no flow will develop in this case since no relative vorticity is applied at the boundaries.

Dr. Glenn Ierley has run his numerical model for the choice $Q_{n}=\beta L / 3$ and $Q_{s}=-\beta L$ which corresponds to the point $n=1 / 3$ and $s=-1$ in Fig. 4.11. Fig. 4.10 shows the steady state $\psi$ and $q$ fields obtained. There is a narrow gyre pressed against the northern wall and a very weak diffusive flow filling the rest of the box. The gyre fills only part of the domain and selects its own boundary. A schematic picture of the flow regimes in the inviscid limit is depicted in Fig. 4.12: the recirculation fills only a portion of the basin from $y_{s}$ to $L$, and south of $y_{s}$, the fluid is at rest.

Because the southern edge of the gyre is not a solid boundary but a free streamline, some constraints need to be imposed on the flow. Specifically, the tangential velocity has to be continuous across the free streamline $y_{s}$ and therefore zero. If this condition was not met, there would be, in the inviscid limit, an infinite amount of relative vorticity which would violate the result that no shear layers are admitted (see the discussion following Eq. 4.2.4).

It is important to notice that although velocities are continuous, potential vorticity will experience a discontinuity across $y_{s}$, smoothed by the diffusion over a distance decreasing with diffusion.

Because tangential velocity is zero on $y_{s}$, the boundary value of $q$ at that location does not affect the "velocity weighted" average (4.3.2). Therefore, if the aspect ratio $\epsilon$ is small, the homogenized value of $q$ will be very close to the northern boundary value 


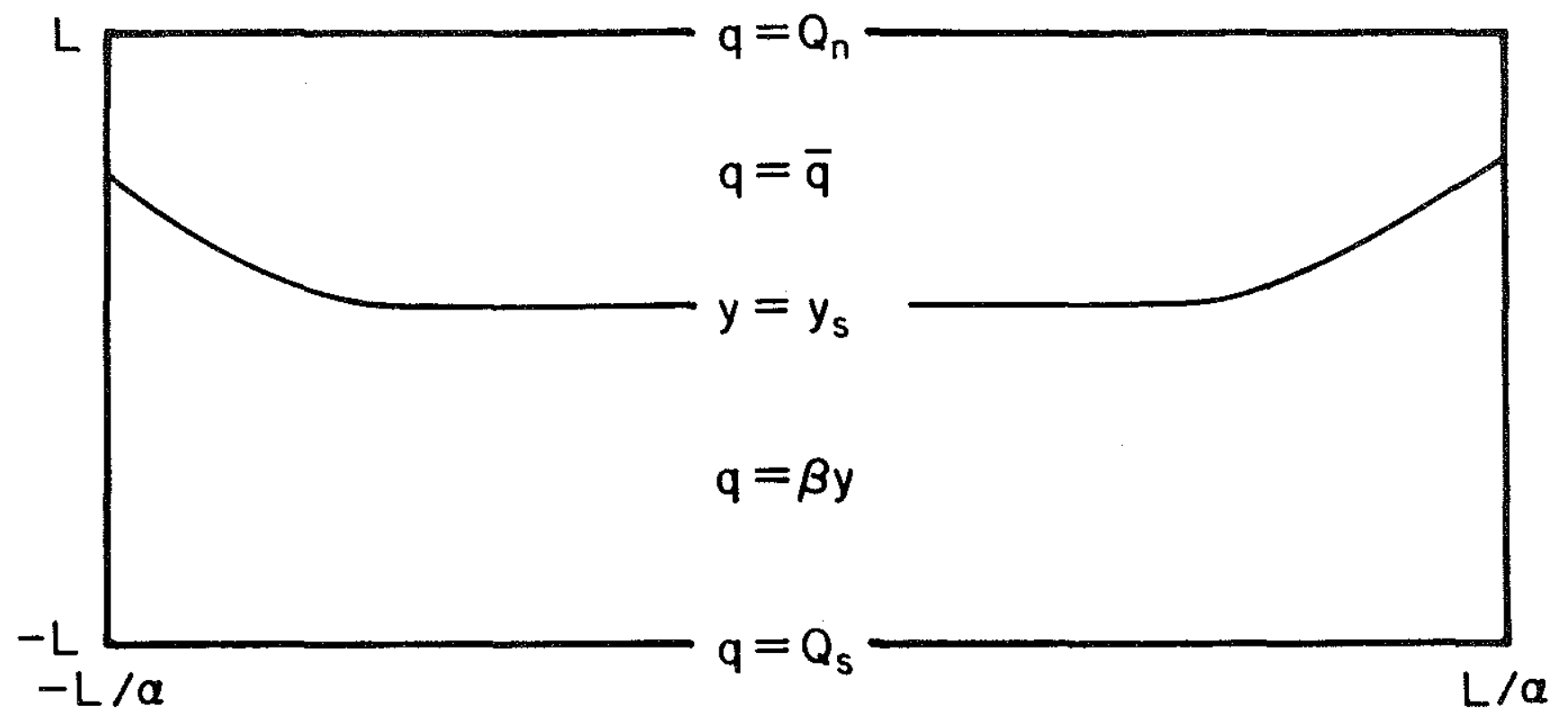

Fig. 4.12. Schematic picture of Fig. 4.10, showing the flow regimes in different regions. South of the free streamline $y=y_{s}$ the fluid is at rest. North of $y=y_{a}$ the fluid circulates with uniform potential vorticity. At the free streamline, $y_{s}$, both $\psi$ and $\vec{\nabla} \psi$ are zero. 
$Q_{n}$, and indeed, in Fig. 4.10 where $Q_{n}=\beta L / 3$ and $Q_{s}=-\beta L$ potential vorticity inside the gyre homogenizes to $q \simeq 0.28 \beta L$.

Furthermore, the condition that the southern edge of the gyre is a free streamline sets a relation between $\bar{q}$ and the meridional scale of the gyre. This relation, together with (4.3.2) determines completely the problem of finding $y_{s}$ and $\bar{q}$ once potential vorticity is specified on the western, northern, and eastern walls.

The solution of Poisson equation (4.3.3) in the case where part of the boundary is an unknown streamline on which $\psi$ and $\vec{\nabla} \psi$ vanish is a very hard problem in an arbitrary geometry. In the following, only approximate solutions will be given under the simplifying assumptions that the meridional scale of the gyre is much smaller than the longitudinal scale $(\epsilon \ll 1)$. In this approximation, the problem becomes onedimensional away from the eastern and western walls, and (4.3.3) reduces to

$$
\psi_{y y}+\beta y=\bar{q}
$$

with boundary conditions $\psi=0$ on $y=L, y_{s}$ and additionally,

$$
\psi_{y}=0 \text { on } y=y_{s} .
$$

There are thus three boundary conditions applied on a second order differential equation. The problem has a solution because one of the boundaries, $y_{s}$, is unknown. The relationship between $y_{s}$ and $\bar{q}$ can be found immediately by multiplying (4.4.1) by $(y-L)$ and integrating in $y$ from $y_{s}$ to $L$.

$$
\int_{y_{0}}^{L} d y(y-L) \psi_{y y}=\int_{y_{0}}^{L} d y(y-L)(\bar{q}-\beta y)
$$

The left-hand side vanishes when all the boundary conditions are applied and performing the trivial integrals on the right-hand side, we find

$$
\left(L-y_{s}\right)=\frac{3}{2}\left(L-\frac{\bar{q}}{\beta}\right)
$$


Dr. W. Young gave an independent derivation of this result by solving (4.4.1) and applying all the boundary conditions. He found

$$
\begin{gathered}
\psi=-\beta(y-L)\left(y-y_{s}\right)^{2} / 6 \\
\bar{q}=\beta\left(L+2 y_{s}\right) / 3
\end{gathered}
$$

In the same approximation of an infinitesimal aspect ratio $\epsilon$ of the box, the "average" value of potential vorticity is given simply by

$$
\bar{q}=Q_{n}
$$

and (4.4.3) can be written as

$$
\left(L-y_{s}\right)=\frac{3}{2 \beta}\left(\beta L-Q_{n}\right)
$$

This shows that the meridional extent of the gyre is proportional to the relative vorticity $Q_{n}-\beta L$ applied at the northern wall, and is independent of the meridional extent of the box.

For this one-dimensional solution, the maximum transport obtained with (4.4.4) can be related to the meridional extent of the gyre, and is given by

$$
\psi_{\max }=\frac{2}{81} \beta\left(L-y_{s}\right)^{3}
$$

Notice that this relation depends only implicitely on the value of $\bar{q}$ and the transport predicted by (4.4.6) can be computed using th observed gyre width, a quantity much easier to observe than the potential vorticity boundary condition. This result completes the analogy with the wind-driven experiments analyzed in the introduction. As the forcing gets stronger $\left(Q_{n}\right.$ is decreased from $\left.\beta L\right)$ the recirculating gyre expands, the homogenized value of potential vorticity decreases and the transport of the gyre increases. In Fig. 4.13 the zonal velocity profile calculated from (4.4.4) is shown. The region of westward flow is twice as wide as the region of eastward flow, and the maximum eastward velocity is three times larger than the maximum westward velocity. 


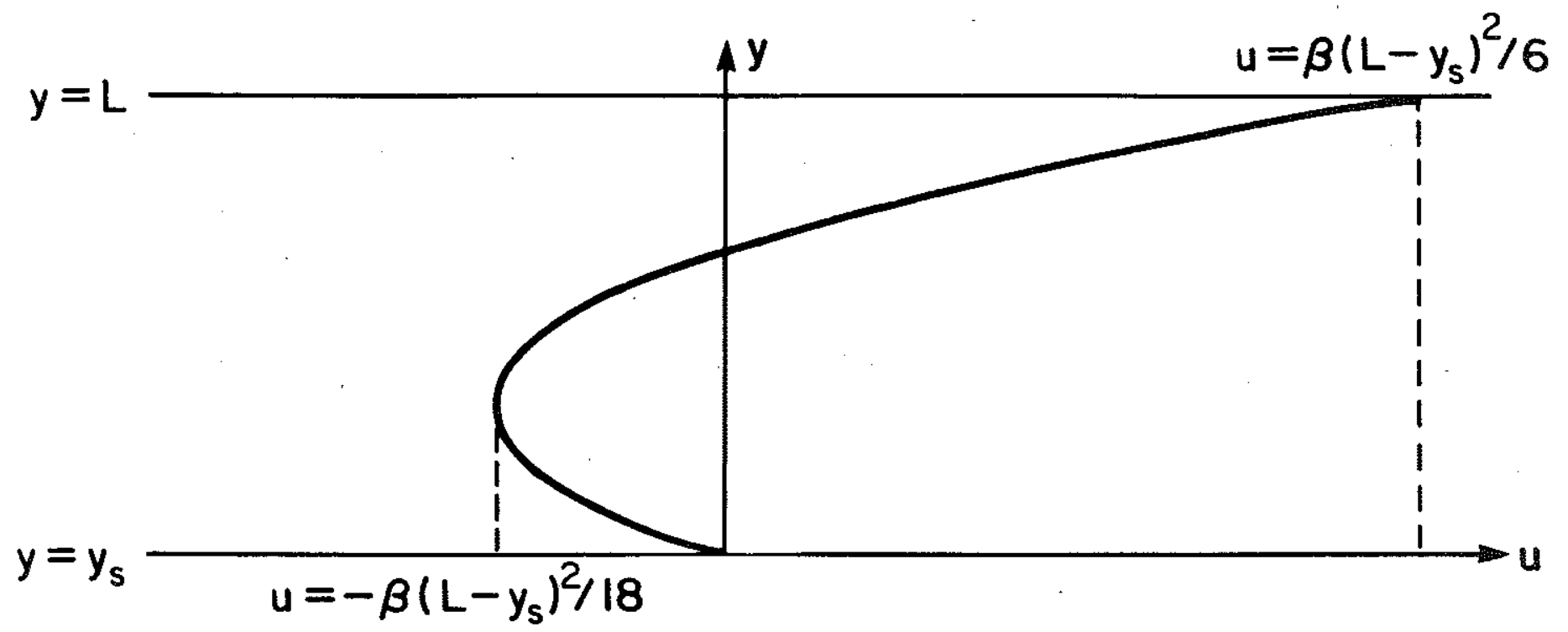

Fig. 4.13. Zonal velocity profile resulting from (4.4.4a). The minimum velocity occurs at $y-y_{o}=\left(L-y_{0}\right) / 3$ and the velocity changes sign at $y-y_{\bullet}=2\left(L-y_{s}\right) / 3$. 
Comparison of the analytical solution obtained in the limit of infinitesimal aspect ratio $\epsilon$ with the numerical solution obtained by Dr. Ierley for finite $\epsilon$ shows very good agreement. For the case shown in Fig. 4.9, $Q_{n}=-\beta L / 3$ and $Q_{s}=-\beta L$, the values for the southern edge of the gyre predicted from (4.4.5) is $y_{s}=-L$, that is the recirculating gyre just fills the whole box and the southern wall is actually a free streamline. The corresponding prediction for $\bar{q}$ is then $\bar{q}=-\beta L / 3$. The numerical run indicates a value for $\bar{q} \simeq-0.4 \beta L$. For the case shown in Fig. $4.10 Q_{n}=\beta L / 3, Q_{s}=-\beta L$ and the approximated formulas give $\bar{q}=\beta L / 3$ and $y_{s}=0$, i.e., the gyre fills half the box. The numerical experiments indicates a value of $\bar{q}=0.28 \beta L$ and the meridional extent of the gyre is in good agreement as well.

\subsection{A Comment on the Zonal Scale of the Recirculation}

In the previous discussion, the recirculating gyre fills the whole box in the zonal direction. To ensure that this is the case, the forcing, $q_{b}(s)$, has been chosen constant on the northern wall. But, indeed, both in oceanic observation and in wind-driven ERGCM's this is not the case (see for example Fig. 4.3). The gyre is confined to the northwest corner of the subtropical gyre. It is conceivable that if the boundary forcing decays eastward as well as southward, the inertial gyre will be confined to the northwest corner of the basin. Unfortunately, with the present formulation of the model, where dissipation is infinitesimal and the Sverdrup interior surrounding the gyre is neglected, a gyre which does not fill the basin in the zonal direction cannot be supported. The proof will be outlined in the following.

Consider a gyre pressed against the northern $(y=0)$ and western wall $(x=$ 0) with a southeastern boundary given by $y_{s}(x)$ (see Fig. 4.14). In the inviscid limit, potential vorticity will be homogeneous inside the gyre and (4.3.3) holds. Integrating (4.3.3) over the area enclosed by the gyre, we find

$$
\oint \vec{u} \cdot d \vec{\ell}=\int d A(\bar{q}-\beta y) .
$$


We are looking for a solution consisting of one anticyclonic gyre where the circulation $(\oint \vec{u} \cdot d \vec{\ell})$ is negative. In this geometry, $\beta y$ is always negative, therefore, $\bar{q}$ must be negative. Multiplying (4.3.3), by $x y$ and integrating over the area enclosed by the gyre, we find

$$
\oint x y \vec{u} \cdot d \vec{\ell}=\int d A x y(\bar{q}-\beta y)
$$

The left-hand side is zero because the integral is performed along the contour $x=0$, $y=0$ and $y=y_{s}(x)$ where the tangential velocity is zero. If $y_{s}\left(x_{0}\right)$ is the southernmost point of the recirculating gyre, it can easily be shown from (4.5.2) that

$$
\beta y_{s}\left(x_{0}\right) \leq \frac{3}{2} \bar{q}<\bar{q}
$$

Therefore, $\bar{q}-\beta y$ changes sign inside the gyre. Let's now consider a system of coordinates $s, n$ such that $s$ is along the streamlines and $n$ is normal to the streamlines. Then by definition $\partial^{k} \psi /\left(\partial s^{k}\right)=0$. At any point $s, n$ located just inside the gyre, and close to a point $s_{0}, n_{0}$, located on the outer streamline $\psi=0$, we have, expanding in a Taylor series

$$
\left.\psi(s, n) \simeq \frac{\partial \psi}{\partial n}\right|_{n_{0}, \theta_{0}}\left(n-n_{0}\right)+\left.\frac{1}{2} \frac{\partial^{2} \psi}{\partial n^{2}}\right|_{n_{0}, \theta_{0}}\left(n-n_{0}\right)^{2}
$$

On the free streamline, $\partial \psi /(\partial n)$ is zero, and, if diffusive effects can be neglected, $\nabla^{2} \psi=\bar{q}-\beta y$ and thus

$$
\psi(s, n) \simeq \frac{\left(n-n_{0}\right)^{2}}{2|\vec{\nabla} n|^{2}}(\bar{q}-\beta y)
$$

Thus $\psi(s, n)$, in the vicinity of the outer streamline, changes sign at the latitude $y=\bar{q} / \beta$, and the possibility of having a single gyre circulation is ruled out.

This proof does not rigorously rule out the possibility of multiple gyres with different values of homogeneous potential vorticity. But a multiple gyre solution would not be physically relevant to the recirculation problem. 


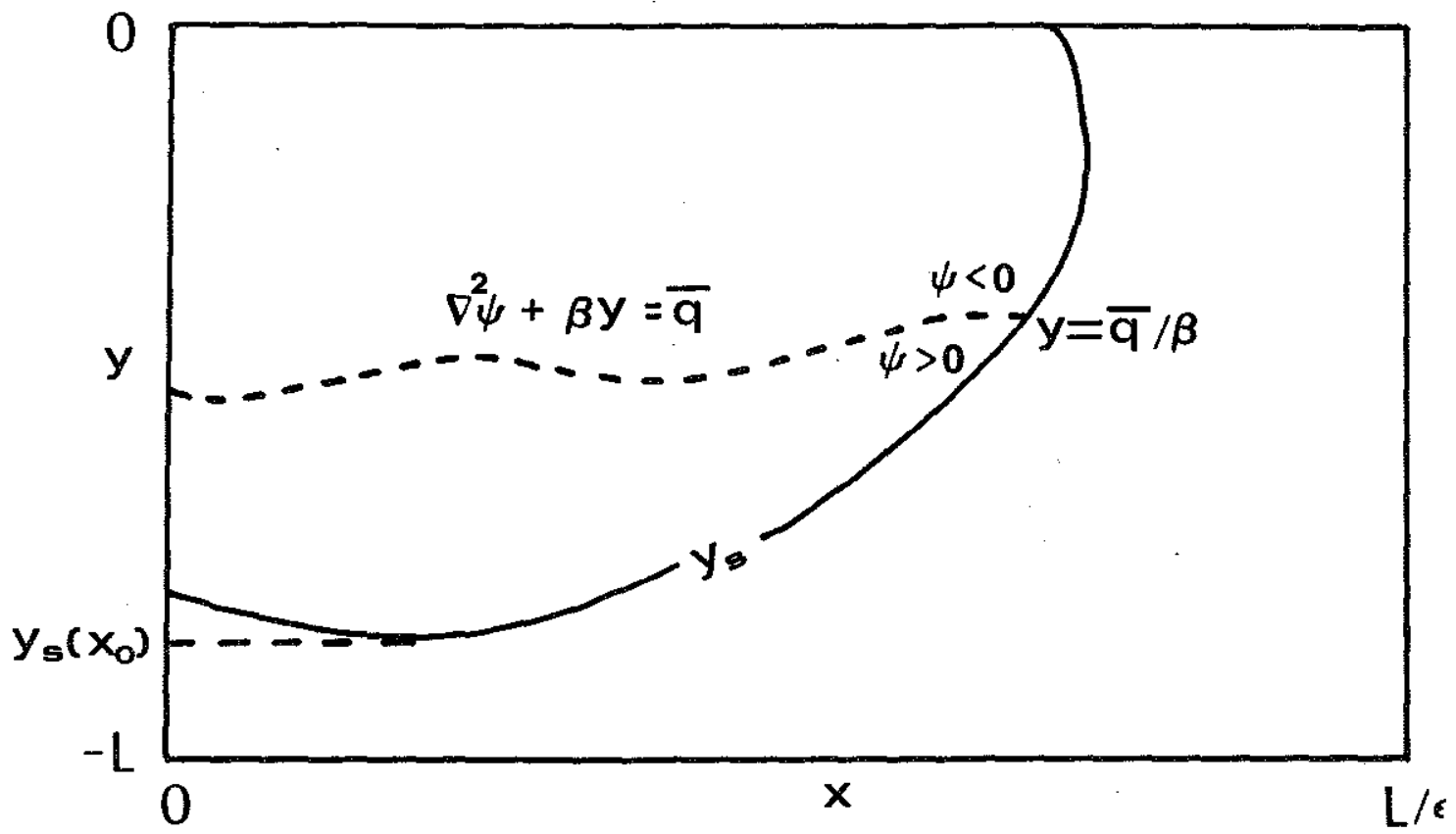

Fig. 4.14. Schematic picture of the gyre geometry when the recirculation is confined to the northwest corner of the box (as in Fig. 4.3). The curve $y=y_{e}(x)$ is a free streamline where $\psi=\vec{\nabla} \psi=0$. In the vicinity of the point $y_{s}(x)=\bar{q}$ the circulation
changes sign. 
Clearly, the results used in the previous argument do not hold if the gyre hits the eastern wall as well as the western wall, and a single anticyclonic gyre may exist in that case, as long as the free streamline is located south of $y=\bar{q}$. In the case where the gyre fills the basin in the zonal direction, the correction due to diffusion and to the matching of velocities with the Sverdrup interior can be neglected. If one seeks a solution for a gyre which is confined to the northwest corner of the basin, diffusive effects or the matching with the Sverdrup interior are of primary importance. Ierley and Young (1987) analyzed the problem of a gyre forced by a potential vorticity boundary anomaly confined to the northwest corner of a rectangular box, in the absence of any Sverdrup interior. For moderate values of diffusivity, $\kappa$, a gyre is obtained pressed against the northwest corner where the forcing is applied. As diffusivity is reduced, the gyre shrinks in the meridional direction and expands in the zonal direction until the eastern boundary is eventually reached and the shrinking of the gyre is arrested. 


\subsection{Summary and Discussion}

One of the prominent features of the recirculating gyre responsible for the increased transport of the separated Gulf Stream is that it has a strong barotropic structure. This property is confirmed by results from purely wind-driven ERGCM's. Therefore to a first approximation, some of its characteristics can be analyzed with a barotropic model.

The analysis of some wind-driven barotropic numerical models suggests that the recirculation can be modeled as an inertial gyre with constant potential vorticity. In a barotropic model this implies that relative vorticity is as large as planetary vorticity everywhere in the gyre, both in the eastward and in the westward flow. The local input of vorticity by the wind can be neglected to a first approximation. Instead, the forcing is provided by prescribing anomalous values of potential vorticity at the rim of the recirculating gyre. With this prescription, the constant value of potential vorticity can be calculated without first explicitly solving for the flow inside the gyre. In particular, the constant value of $q$ is given by the average of the boundary values weighted by the velocity on such boundaries.

In all the cases presented, the recirculating gyre fills the basin in the zonal direction. Therefore, it is consistent to isolate the gyre from the Sverdrup interior and to neglect diffusive effects.

For strong boundary forcing, the gyre fills the box in the meridional direction as well and the width of the gyre does not emerge as a prediction of the model. For weak forcing, the gyre is confined to a subregion and the meridional width of the gyre can be calculated as well as its homogenized value of potential vorticity. The latter case is the most relevant to the oceanic recirculation. Explicit solution were derived numerically by Dr. Ierley, and, in the limit of a long and narrow gyre, analytically by Dr. Young and myself. The analytic solution shows that the width of the gyre is proportional to the forcing applied at the northern boundary, and that the maximum transport is 
proportional to the cube of the width. This result is consistent with the finding of wind-driven numerical models that, as the wind forcing/dissipation ratio increases, the gyre expands and its strength increases. For a gyre $450 \mathrm{~km}$ wide and $2700 \mathrm{~m}$ deep, the analytic solutions gives a maximum transport of 121.5 Sverdrup which is in very good agreement with the results of the baroclinic ERGCM of Robinson et al. (1977).

A number of questions remain open. The first is to explain why both in baroclinic ERGCM's and in the ocean, the recirculation has a weakly depth-dependent structure. This problem is addressed in the next chapter where the calculations of section 4.4 are extended to a two-layer model. The second problem is to formulate a model where the longitudinal extent of the gyre is predicted as well as its latitudinal width. This question can be addressed by choosing a boundary forcing which depends on $x$ as well as $y$. As remarked in section 4.5, in order to address this issue, the matching with the Sverdrup interior or the effects of diffusion (or both) must be included. Drs. Ierley and Young are presently analyzing the case where diffusive effects are taken into account in the absence of a Sverdrup interior.

The last and hardest question is how to relate the boundary values of potential vorticity to the global wind forcing and dissipation or to the diabatic processes which are supposed to be responsible for the anomalous potential vorticity at the edge of the recirculation. This is an important problem which deserves attention in the future but will not be addressed in this thesis. 


\section{Appendix A4 - Derivation of the "Velocity Weighted" Average, (4.3.2)}

The result (4.3.2) holds if instead of potential vorticity we consider the more general problem of a generic passive tracer which satisfies the advection diffusion equation

$$
\begin{aligned}
J(\psi, \theta) & =\kappa \nabla^{2} \theta \\
\psi & =0, \theta=\theta_{b} \quad \text { on the boundary }
\end{aligned}
$$

Multiplying by $\psi$ and integrating over the area of the box we eventually obtain

$$
\int d A \theta \nabla^{2} \psi=\oint \theta_{b} \vec{u}_{b} \cdot d \vec{\ell}
$$

In the limit of small diffusion, $\theta$ will be given by its homogenized value $\bar{\theta}$ everywhere except in narrow boundary layers close to the edge of the gyre. Therefore, in this strongly advective limit, the left-hand side of (A4.2) can be approximated with

$$
\int d A \theta \nabla^{2} \psi \simeq \bar{\theta} \int d A \nabla^{2} \psi
$$

and (A4.2) becomes

$$
\vec{\theta}=\frac{\oint \theta_{b} \vec{u}_{b} \cdot d \vec{\ell}}{\oint \vec{u}_{b} \cdot d \vec{\ell}}
$$

The result is implicit in Roberts' (1977) work. His derivation made use of the local coordinates to express the dominant balance in (A4.1), while the integral balance (A4.4) (proved in conjunction with Dr. W.R. Young) provides an explicit formula for the interior homogenized value of the tracer concentration. The velocity weighted average (A4.4) is confirmed by the numerical experiment of Musgrave (1985). In his work he showed that a passive tracer satisfying (A4.1), in the presence of a western intensified flow, would homogenize in the interior to the value on the western boundary. Interestingly enough, the derivation that leads to (A4.4) can be used for potential vorticity which is not a passive tracer. Because of the extremum principle (see the discussion following Eq. 4.2.4) the relative vorticity is bounded by its boundary values and so is 
finite everywhere in the domain, including the boundary layers. Therefore, the approximation (A4.3) holds for potential vorticity as well as for a passive tracer. Moreover, the velocity at the boundary, $\vec{u}_{b}$, can be approximated with the interior velocity just outside the boundary layer and this allows the complete determination of the homogenized value of potential vorticity without a detailed boundary layer solution.

\section{Appendix B4 - How to Find $\bar{q}$ Without Solving for the Flow}

The velocity weighted average (4.3.2) can be written as

$$
\bar{q}=\frac{\oint q_{b}(\vec{\nabla} \psi) \cdot \hat{n} d \ell}{\oint \vec{\nabla} \psi \cdot \hat{n} d \ell}
$$

Since $q_{b}$ is only defined on the boundary, we have the freedom to define a function $g$ everywhere in the domain such that

$$
\begin{aligned}
\nabla^{2} g & =0 \text { in the interior and } \\
g & =q_{b} \text { on the boundary }
\end{aligned}
$$

With this choice and using Gauss' theorem, $\oint q_{b}(\vec{\nabla} \psi) \cdot \hat{n} d \ell$ becomes $\int d A g \nabla^{2} \psi$ and the expression for the averaged potential vorticity is

$$
\bar{q}=\frac{\int d A g(\bar{q}-\beta y)}{\int d A(\bar{q}-\beta y)}
$$

where $g$ is a known function, independent of $\psi$, which satisfies (B4.1). 


\section{Appendix C4 - Homogenized gyre filling an elliptical domain}

In this section the rectangular box will be replaced by an elliptical domain, where solutions to Eq. 4.3.3. can be found analytically. The solution to (4.3.3) with boundary conditions $\psi=0$ on $\epsilon^{2} x^{2}+y^{2}=L^{2}$ is given by

$$
\psi=\frac{\left(\epsilon^{2} x^{2}+y^{2}-L^{2}\right)}{2}\left(\frac{\bar{q}}{\epsilon^{2}+1}-\frac{\beta y}{3+\epsilon^{2}}\right) .
$$

Clearly, this solution gives a solution consisting of one gyre only if

$$
\left|\frac{\bar{q}}{\beta L}\right| \frac{3+\epsilon^{2}}{\epsilon^{2}+1}>1 \text {. }
$$

To determine the value of $\vec{q}$ in the interior of the ellipse, we use (4.3.4) for the boundary forcing given by (4.3.1). The solutions for an elliptical domain corresponding to (4.3.5) are given by

$$
\bar{q}=\frac{1}{4}\left\{Q_{n}+Q_{s} \pm \sqrt{\left(Q_{n}+Q_{s}\right)^{2}-2 \beta L\left(Q_{n}-Q_{s}\right)}\right\} .
$$

Here $Q_{n}$ and $Q_{s}$ are the values of $q$ at $y=L$ and $y=-L$ respectively.

Again, the value of $\bar{q}$ is independent of the aspect ratio $\epsilon$ which enters in the constraint (C4.1). For each value of $n=Q_{n} /(\beta L)$ and $s=Q_{s} /(\beta L)$, a consistency check must be made on the value of $\bar{q}$ given by (C4.2). The value of $\bar{q}$ has to be within the boundary values, and it must satisfy (C4.1). In Fig. C4 the values of $\bar{q}$ resulting from (C4.2) are plotted as a function of $Q_{n}$ for a fixed value of $Q_{s}=-\beta L$. Only one root is plotted since the other root gives values that are outside the boundary values. The constraint (C4.1) is shown on the right side of the plot for discrete values of the aspect ratio $\epsilon$. 


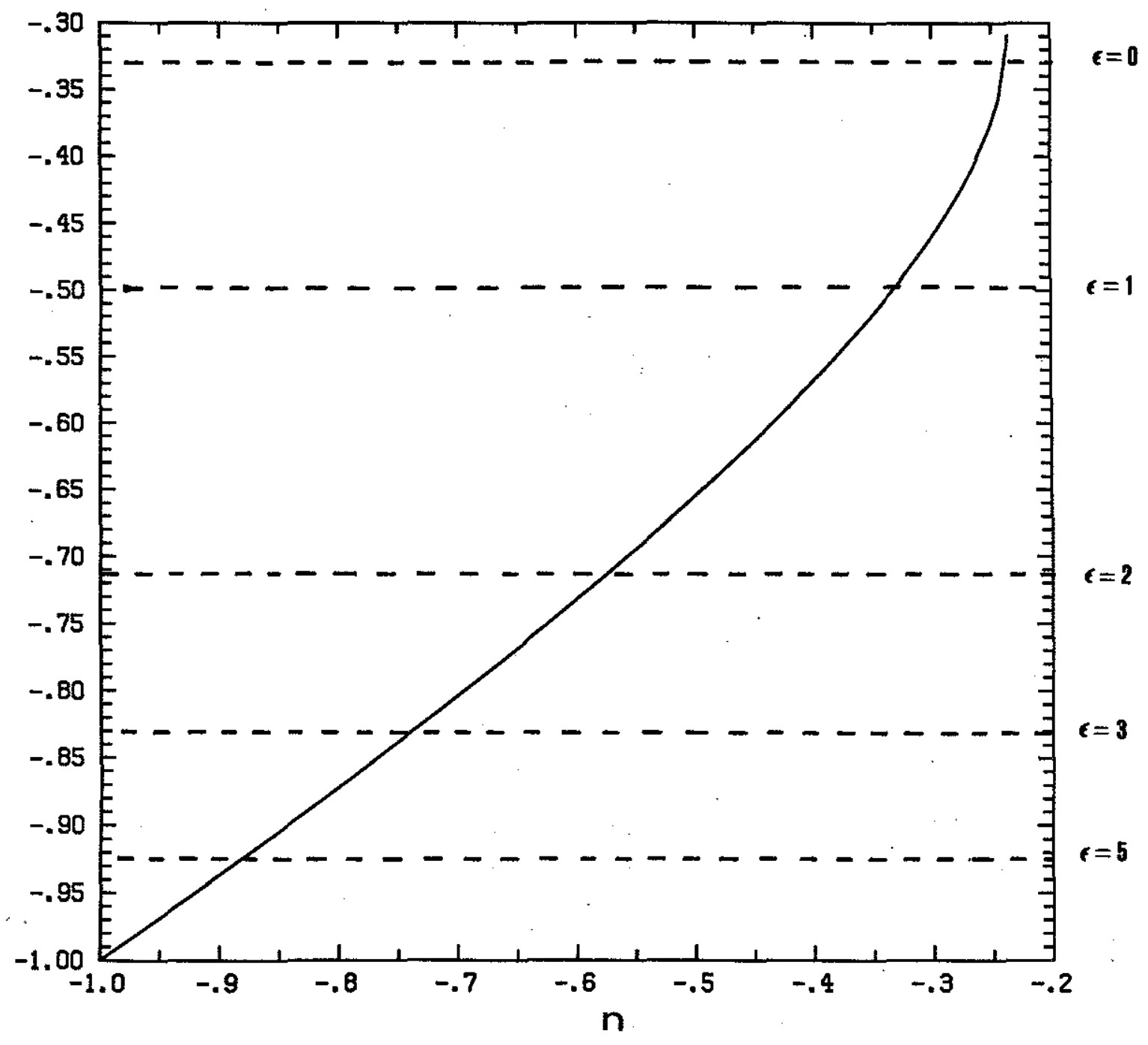

Fig. C4. $\bar{q} / \beta L$ resulting from the solution of (C4.2) as a function of $n=Q_{n} / \beta L$, for a fixed value of $Q_{s}=-\beta L$. The constraint (C4.1) is shown on the right hand side of the graph for discrete values of the aspect ratio $\epsilon \cdot \bar{q} / \beta L$ should be below the dashed line in order to satisfy (C4.1). 


\section{CHAPTER 5 \\ A model of the inertial recirculation \\ driven by boundary currents: \\ the stratified model}

\section{Summary}

An inertial gyre with characteristics very similar to the recirculation observed in eddy resolving general circulation models is obtained with a simple, analytically tractable, two-layer model. The recirculating gyre is contained in a box of simple geometry which isolates it from the Sverdrup interior. The gyre is forced by prescribing anomalous values of potential vorticity at the edge of the box. This mimics the effect of the western boundary current carrying low values of potential vorticity northward in the subtropical gyre or can be thought of as a rough parameterization of diabatic forcing. In both cases the forcing is confined to the thermocline waters, which are represented by the upper layer. Therefore the boundary forcing is confined to the upper layer and is transmitted to the abyssal ocean through interfacial stresses.

The condition for the abyssal water to be set in motion is derived and for oceanic values the recirculation goes all the way to the bottom. When this occurs the center of the gyre is dominated by a barotropic flow, while the baroclinic flow is confined to the edges of the gyre. Analogously to the barotropic model considered in the previous chapter, the width and strength of the gyre can be easily calculated in the limit of long, narrow gyres. The meridional scale of the gyre is directly proportional to the vorticity anomaly injected at the northern boundary, and the barotropic part of the transport is proportional to the cube of the abyssal gyre width, in close analogy with the results found in a one-layer model. 


\subsection{Introduction}

In the previous chapter, the observed vertical coherence of the inertial gyre, responsible for the enhanced Gulf Stream transport, has been exploited to formulate a simple barotropic model of the recirculation. In that work the recirculation appears as an inertial gyre with constant potential vorticity, driven by anomalous low values of potential vorticity applied at the edge of the flow. This boundary forcing mimics the effect of the boundary currents, or perhaps diabatic forcing, producing low values of potential vorticity at the northern edge of the subtropical gyre. With this prescription it is possible to calculate the homogenized value of potential vorticity as well as the meridional extent of the gyre. Although the results obtained are consistent with findings from baroclinic ERGCM, the barotropic model is unable to answer the question of why the recirculation has a weak depth dependent structure.

The vertical coherence of the North Atlantic circulation is well documented in the literature. Schmitz (1980) analyzed an array of current meters deployed in the western North Atlantic along $55^{\circ}$ W. He found "weakly depth dependent" time averaged currents (mostly zonal) flowing eastward just south of the jet axis and returning westward further south with amplitudes that ranged from 6 to $10 \mathrm{~cm} / \mathrm{sec}$ throughout the water column. Both eastward and westward currents had a horizontal scale of roughly 200 $\mathrm{km}$, and the westward flow was surface and bottom intensified. Richardson (1985) used current meters and surface drifter measurements to produce a vertical section of the mean zonal currents flanking the Gulf Stream. He found that the total eastward transport was about $93 \mathrm{~Sv}$ at $55^{\circ} \mathrm{W}$, that is, about three times larger than that observed in the Gulf Stream at the Florida Straits. The excess $63 \mathrm{~Sv}$ are recirculated north and south of the jet axis. In particular about $29 \mathrm{~Sv}$ are transported westward in a southern countercurrent about $200 \mathrm{~km}$ wide. The vertical coherence of this westward flow is remarkable (see especially his Fig. 6b): the zonal velocities (with the wind drift removed) vary from $4 \mathrm{~cm} / \mathrm{sec}$ at the surface to $6 \mathrm{~cm} / \mathrm{sec}$ at the bottom. 
The same vertical coherence is found in the recirculating gyre in wind driven ERGCM's such as that of Schmitz and Holland (1986). In their experiments, performed with a quasi-geostrophic, eddy resolving, eight level model, the mean zonal velocity along a section cutting the recirculation shows a quasi-barotropic westward flow extending all the way to the floor at $5000 \mathrm{~m}$. No bottom intensification is observed in their calculations, presumably because of the absence of topography. The mean westward velocity is about $10 \mathrm{~cm} / \mathrm{sec}$ and the width of the westward recirculation is about $200 \mathrm{~km}$.

In this chapter the question of why the recirculation is "weakly depth dependent" is addressed. Another question that needs to be addressed is whether relative vorticity or vortex stretching is the dominant vorticity term in the region of westward flow. Marshall and Nurser (1986) (herafter referred as MN) neglect the former, while in the homogeneous model the latter is excluded. If potential vorticity is homogenous within the recirculating gyre (as it is in both the present work and in MN) then, in the absence of vortex stretching, relative vorticity has to be of the same order as planetary vorticity everywhere in the gyre, including the westward flow. Indeed this is the case in Schmitz and Holland's (1986) ERGCM results. Although they don't present explicit diagnostics for the balance of terms in the mean vorticity field, a simple estimate can be inferred from their mean zonal velocities. With $u \sim 10 \mathrm{~cm} / \mathrm{sec}$ and a width of the westward flow of $\sim 200 \mathrm{~km}$, relative vorticity can be estimated with $u_{y} \sim(10 \mathrm{~cm} / \mathrm{sec}) /(100 \mathrm{~km})$ $=10^{-6} \sec ^{-1}$. This has to be compared with $\beta y \sim 2 \times 10^{-6} \sec ^{-1}$, and the ratio of relative to planetary vorticity is $\mathbf{0 . 5}$.

A dynamical balance of this type has to emerge as a consequence of a baroclinic model rather than being set forth as an a priori assumption. With this in view the ideas developed in Chapter 4 will be extended to the simplest possible baroclinic model. 


\subsection{Formulation of the baroclinic model and general results}

All the assumptions made in the previous chapter will be retained in the present formulation except the vertical resolution is increased. They will be briefly repeated here. 1) The recirculating gyre extends to the bottom, which is taken as level. Baroclinic effects are taken into account in the simplest possible way, i.e., a two-layer model. The interface represents the thermocline.

2) The recirculation is steady and the eddy field is parameterized as weak lateral diffusion of potential vorticity.

3) There are no body forces applied to the fluid. Local wind or buoyancy forces are neglected in both layers. This approximation may not be as accurate for the surface layer as it was for the barotropic model. Nevertheless, if the upper layer is not very shallow, it may still be acceptable. In order to neglect the wind we must have:

$$
J\left(\psi_{1}, q_{1}\right) \gg \frac{f_{0} w_{e}}{H_{1}} \quad \text { or } \quad \beta v \gg \frac{f_{0} w_{e}}{H_{1}}
$$

In both the observations and the ERGCM results, the meridional velocity $v$ at the center of the recirculation is much smaller than the zonal velocity $u$. Therefore $v$ can be estimated as $v \approx L_{y} u / L_{x}$ where $L_{y}$ is the meridional scale of the recirculation and $L_{x}$ is its zonal scale. With this estimate the wind can be neglected if

$$
\beta L_{y} u / L_{x} \gg f_{0} w_{e}\left(y=L_{y}\right) / H_{1}
$$

In Schmitz and Holland (1986)'s ERGCM the recirculation occurs near the zero of the wind stress curl, and its zonal scale is of the order of the meridional scale of the Sverdrup gyre. Therefore $f_{0} w_{e}\left(y=L_{y}\right) / H_{1}=f_{0} / H_{1} W \sin \left(\pi L_{y} / L_{x}\right) \simeq \pi L_{y} f_{0} W /\left(L_{x} H_{1}\right)$. With $\beta=2 \times 10^{-11} \mathrm{~m}^{-1} \mathrm{sec}^{-1}, \quad H_{1}=1000 \mathrm{~m}, W=10^{-4} \mathrm{~cm} / \mathrm{sec}$ and $u=10 \mathrm{~cm} / \mathrm{sec}$, we find the ratio of the left hand side to to the right hand side in (5.2.1) is $20 / \pi$ which is a reasonably large number and the wind forcing can be neglected. Local buoyancy forcing is more difficult to estimate since its amplitude is not known from oceanic 
measurements. I will assume that it is not bigger than the wind. As in the previuous chapter the forcing for the inertial gyre will be provided by prescribing anomalous values of potential vorticity at the edge of the recirculation. This is a crude paramerization of the effect of the Gulf Stream carrying vorticity of southern origin northward and eastward in the subtropical gyre.

4) Since my attention is focused on the dynamics of the recirculation only, the inertial gyre is considered isolated from the Sverdrup interior, which, consistent with the neglect of the wind, is set to zero.

The equations defining the model are then the quasigeostrophic equation on a $\beta$-plane:

$$
\begin{gathered}
\frac{\partial q_{1}}{\partial t}+J\left(\psi_{1}, q_{1}\right)=\kappa \nabla^{2} q_{1} \\
\frac{\partial q_{2}}{\partial t}+J\left(\psi_{2}, q_{2}\right)=\kappa \nabla^{2} q_{2} \\
\text { where } \quad \begin{aligned}
q_{1} & =\nabla^{2} \psi_{1}+F_{1}\left(\psi_{2}-\psi_{1}\right)+\dot{\beta}_{y} \\
q_{2} & =\nabla^{2} \psi_{2}+F_{2}\left(\psi_{1}-\psi_{2}\right)+\beta y \\
g^{\prime} & =\frac{\rho_{2}-\rho_{1}}{\rho_{1}} g \\
F_{1} & =\frac{f_{0}^{2}}{g^{\prime} H_{1}} \\
F_{2} & =\frac{f_{0}^{2}}{g^{\prime} H_{2}}
\end{aligned}
\end{gathered}
$$

The boundary conditions are applied at the solid walls of a box which contains the recirculation and are: $\psi_{1}=\psi_{2}=0, q_{1}=q_{1 b}(s), q_{2}=q_{2 b}(s)$ on $y= \pm L, x= \pm L / \epsilon$, where $\epsilon$ is the aspect ratio of the box and $s$ is the arclength along the boundary.

Some general results were obtained in the barotropic model and they apply equally well for each layer separately. 
a) Integrating each equation (5.2.2) over the area enclosed by any closed streamline we obtain, in the steady state

$$
\begin{array}{r}
\kappa \oint_{\psi_{1}} \vec{\nabla} q_{1} \cdot \hat{n}_{1} d \ell=0 \\
\kappa \oint_{\psi_{2}} \vec{\nabla} q_{2} \cdot \hat{n}_{2} d \ell=0 \\
\text { where } \quad \hat{n}_{i}=\frac{\vec{\nabla} \psi_{i}}{\left|\nabla \psi_{i}\right|} \quad i=1,2
\end{array}
$$

with the integrals performed on any closed streamline in that layer. This shows that the total diffusive flux is zero across a streamline. In the limit of weak diffusion this implies that potential vorticity is homogenized (Rhines and Young (1982)).

b) The potential vorticity field is bounded by its boundary values. If this were not the case there would be an extremum in the interior, surrounded by a nested set of closed potential vorticity contours. Integrating (5.2.2) over the area enclosed by any closed potential vorticity contour we get, in the steady state:

$$
\begin{aligned}
& \kappa \oint_{q_{1}}\left|\nabla q_{1}\right| d \ell=0 \\
& \kappa \oint_{q_{3}}\left|\nabla q_{2}\right| d \ell=0
\end{aligned}
$$

which is a contradiction.

This shows that, in the steady state, there are no closed potential vorticity contours, and therefore no maxima or minima of potential vorticity in the interior. Thus potential vorticity in each layer is bounded by its boundary values and there cannot be any shear layers. Then, even in the limit of weak diffusion, velocity has to be continuous everywhere in the fluid. Otherwise relative vorticity would become very large and potential vorticity would exceed the boundary values, contradicting the extremum principle. Although velocity has to be continuous, potential vorticity may not be and indeed in the limit of weak diffusion, potential vorticity becomes discontinuous at internal boundary layers. 
c) Multiplying (5.2.2a) by $H_{1} \psi_{1},(5.2 .2 b)$ by $H_{2} \psi_{2}$, integrating over the area of the basin and observing that

$\kappa\left[\int d A \psi_{1} \nabla^{2} q_{1}=-\oint q_{1} \vec{u}_{1} \cdot d \vec{\ell}+\int d A \beta y \nabla^{2} \psi_{1}+\int d A\left[\left(\nabla^{2} \psi_{1}\right)^{2}+F_{1} \nabla^{2} \psi_{1}\left(\psi_{2}-\psi_{1}\right)\right]\right.$

and similarly for the lower layer, the energy equation is obtained by summing the integrals obtained for each layer.

$$
\frac{\partial}{\partial t} \int E d A=H_{1} S_{1}+H_{2} S_{2}-\kappa \int d A\left[H_{1}\left(\nabla^{2} \psi_{1}\right)^{2}+H_{2}\left(\nabla^{2} \psi_{2}\right)^{2}+H_{1} F_{1}\left(\vec{u}_{1}-\vec{u}_{2}\right)^{2}\right]
$$

$$
\text { where }
$$

$$
\begin{aligned}
& S_{1}=\kappa \oint\left(q_{1 b}-\beta y\right) \vec{u}_{1} \cdot d \vec{\ell} \\
& S_{2}=\kappa \oint\left(q_{2 b}-\beta y\right) \vec{u}_{2} \cdot d \vec{\ell}
\end{aligned}
$$$$
E=\frac{1}{2}\left(H_{1} u_{1}^{2}+H_{2} u_{2}^{2}+H_{1} F_{1}\left(\psi_{1}-\psi_{2}\right)^{2}\right)
$$

Equation 5.2.5 shows that in the steady state the amount of relative vorticity, and of vertical shear, in the fluid is proportional to the vorticity anomaly injected at the boundaries. This result emphasizes the role of $q_{1 b}$ and $q_{2 b}$ as forcing. If both $q_{1 b}=$ $q_{2 b}=\beta y$ then the fluid is at rest in both layers. Notice that if diffusion is completely absent there is neither dissipation nor forcing, and the fluid will preserve its initial state. If dissipation is present, in the steady state, one also finds

$$
\begin{aligned}
& \oint\left(q_{1 b}-\beta y\right) \vec{u}_{1} \cdot d \vec{\ell}+\int d A F_{1} \vec{u}_{1} \cdot \vec{u}_{2}=\int d A\left[\left(\nabla^{2} \psi_{1}\right)^{2}+F_{1} u_{1}^{2}\right] \\
& \oint\left(q_{2 b}-\beta y\right) \vec{u}_{2} \cdot d \vec{\ell}+\int d A F_{2} \vec{u}_{1} \cdot \vec{u}_{2}=\int d A\left[\left(\nabla^{2} \psi_{2}\right)^{2}+F_{2} u_{2}^{2}\right]
\end{aligned}
$$

Therefore if only one layer is forced at the boundaries, there may still be motion in the unforced layer due to the "drag" exerted at the interface. It is worth observing that, in this case, $\vec{u}_{1}=\vec{u}_{2}=0$ is not a solution of (5.2.2) unless $q_{1 b}=q_{2 b}=\beta y$.

Although these general results are very instructive, and many properties of the flow can be deduced just from the analysis of integral properties, explicit solutions of the problem expressed by (5.2.2) for arbitrary values of diffusion can only be obtained 
numerically. I will restrict my analysis to the limit of weak diffusion, which is probably the most relevant for the oceanic recirculation.

The anomalous value of potential vorticity at the rim of the gyre is supposed to mimic the effect of the western boundary current carrying low values of planetary vorticity northward in the wind driven ERCGM's, or can be thought of as a crude parameterization of the mode water formation occurring within the thermocline. In both cases the direct forcing is exerted in the upper layer and transmitted to the abyssal ocean through mesoscale processes which, in my model, are parameterized as diffusion of potential vorticity. Therefore in all cases presented, I have forced the upper layer but not the lower layer $\left(q_{2 b}=\beta y\right)$. This choice is consistent with results from wind-driven ERCGM's, such as that of Holland et al.'s (1984), and with analysis of North Atlantic data, such as that of Bower et al.'s (1985). In Holland et al.'s (1984) model, strong potential vorticity gradients across the separated Gulf Stream ( $y=L$ in the present model) are observed in the layer directly forced by the wind. Similarly, Bower et al. (1985) show that, in the surface water $\left(\sigma_{\theta}<27.0\right)$, potential vorticity, together with other tracers, exhibits huge gradients across the separated Gulf Stream. According to the present model's interpretation, this jump in properties is due to the confluence of low values of potential vorticity generated in the south and advected northward and eastward by the Gulf Stream in the subtropical gyre, with high values of potential vorticity generated in the north and advected southward and eastward by the Gulf Stream in the subpolar gyre. On the other hand, in layers shielded from the wind forcing potential vorticity is constant across the separated Gulf Stream in Holland et al.'s (1984) ERGCM. This result is supported by the observations of Bower et al. (1985), where potential vorticitybelow the $27.0 \sigma_{\theta}$ surface is rather homogeneous across the separated Gulf Stream. According to my interpretation, the lower layer of my model lies below the thermocline, where no direct forcing is applied and therefore there is no anomalous potential vorticity generation. 
As in Chapter 4 only gyres that fill the whole basin in the zonal direction will be analyzed. This is so because for simplicity my attention is restricted to boundary forcing which is independent of longitude. The simplest choice for the potential vorticity forcing is

$$
\begin{aligned}
& q_{1 b}=\frac{\left(Q_{n}-Q_{s}\right)(y-L)}{2 L}+Q_{n} \\
& q_{2 b}=\beta y
\end{aligned}
$$

where $Q_{n}$ and $Q_{s}$ are the constant values of $q_{1}$ on the northern and southern boundaries respectively. Because the recirculating gyre is supposed to rejoin the Sverdrup interior at its southern boundary, $Q$, has been chosen as $-\beta L$ in all cases presented. Numerical solutions of (5.2.2) obtained with the numerical model developed by Dr. Ierley are shown in Figs. 5.1, 5.2 and 5.3 for different values of the forcing $Q_{n}$ and of the depth ratio. In all cases presented the gyre in the lower layer is contained within the region of motion of the upper layer and potential vorticity is homogeneous in both gyres. Homogenization should be expected from the result (5.2.3) in the limit of weak diffusion. As proved in Appendis A4 the homogenized value of potential vorticity in each gyre is given by

$$
\begin{aligned}
\bar{q}_{1} & =\frac{\oint q_{1 b} \vec{u}_{1} \cdot d \vec{\ell}}{\oint \vec{u}_{1} \cdot d \vec{\ell}} \\
\bar{q}_{2} & =\frac{\oint q_{2 b} \vec{u}_{2} \cdot d \vec{\ell}}{\oint \vec{u}_{2} \cdot d \vec{\ell}}
\end{aligned}
$$

where the integrals are performed along the boundaries of the gyres.

This remarkable result has a simple physical explanation already given in Chapter 4, which will be repeated here. As the fluid is advected along the streamlines, potential vorticity is diffused across the streamlines. Diffusion is more efficient where the streamlines are closer, that is where velocities are larger (Roberts, 1977). This is why, in (5.2.7), the boundary values of potential vorticity are weighted by the velocity. A detailed derivation of this result can be found in Appendix A4. 
(a)

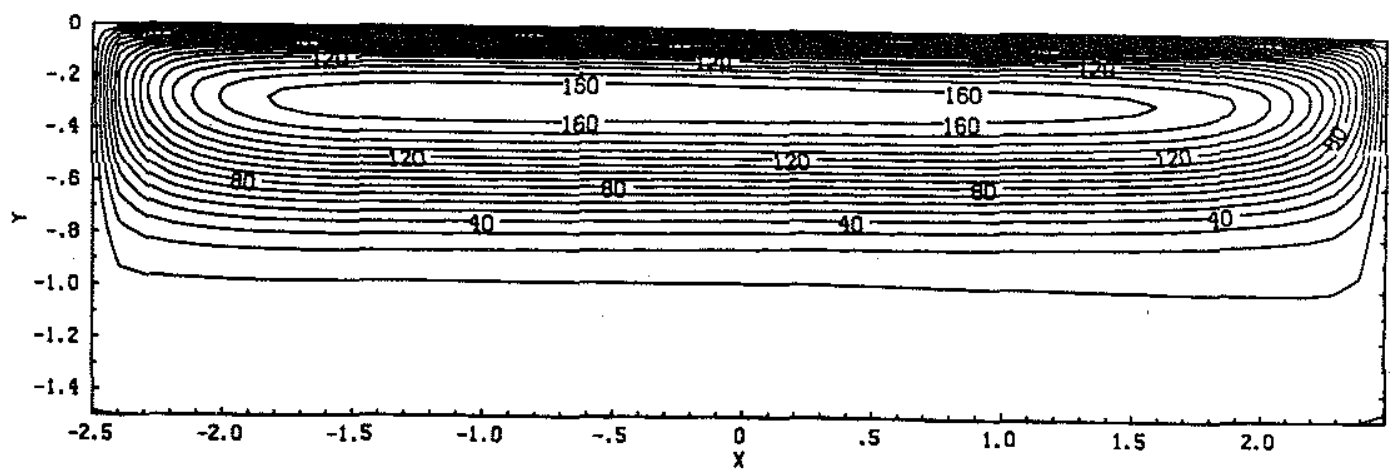

(b)

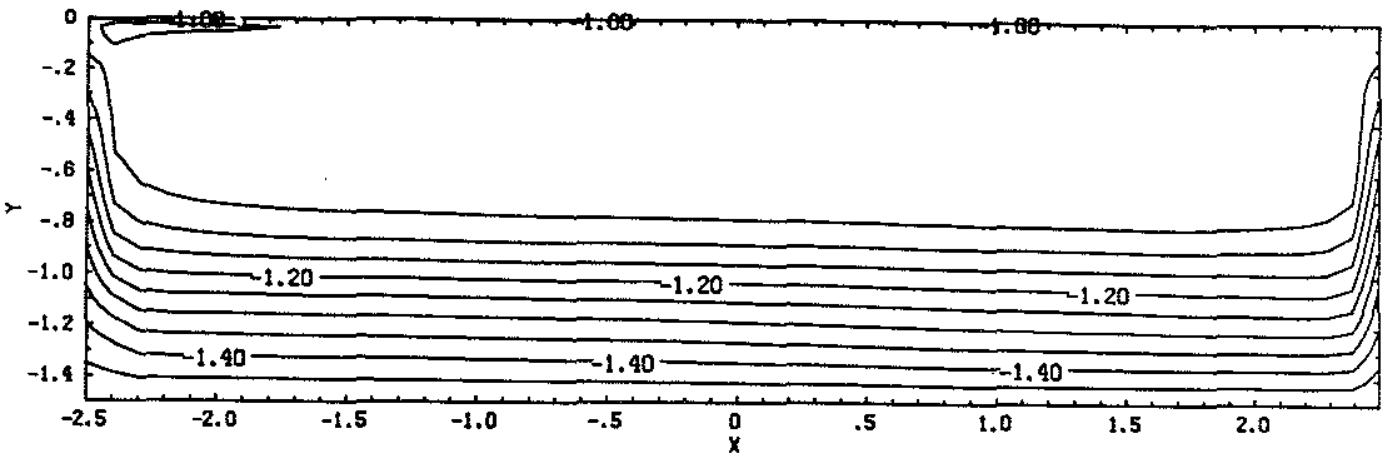

(c)

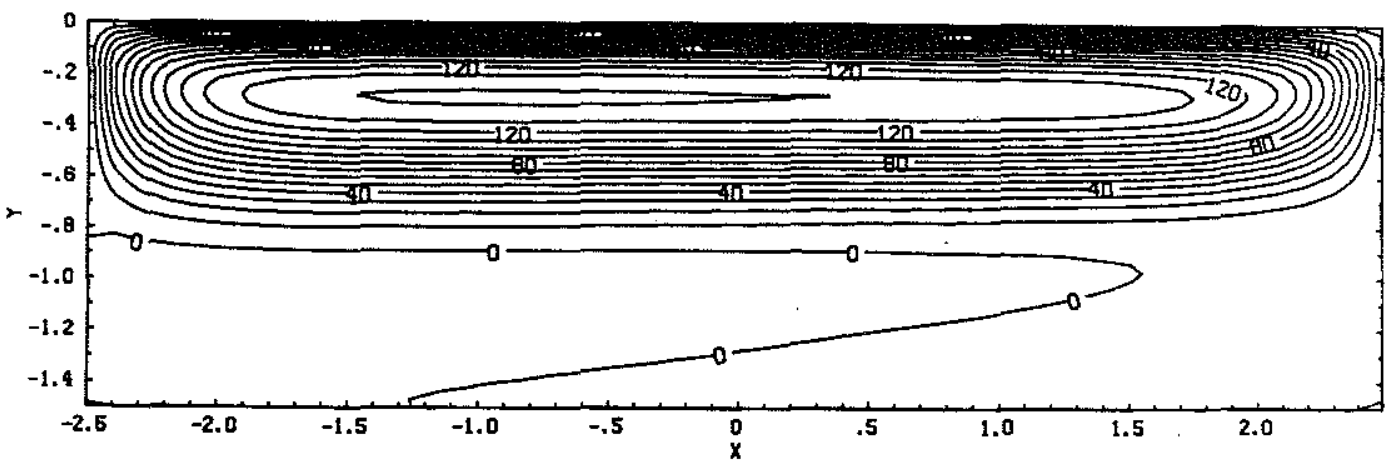

(d)

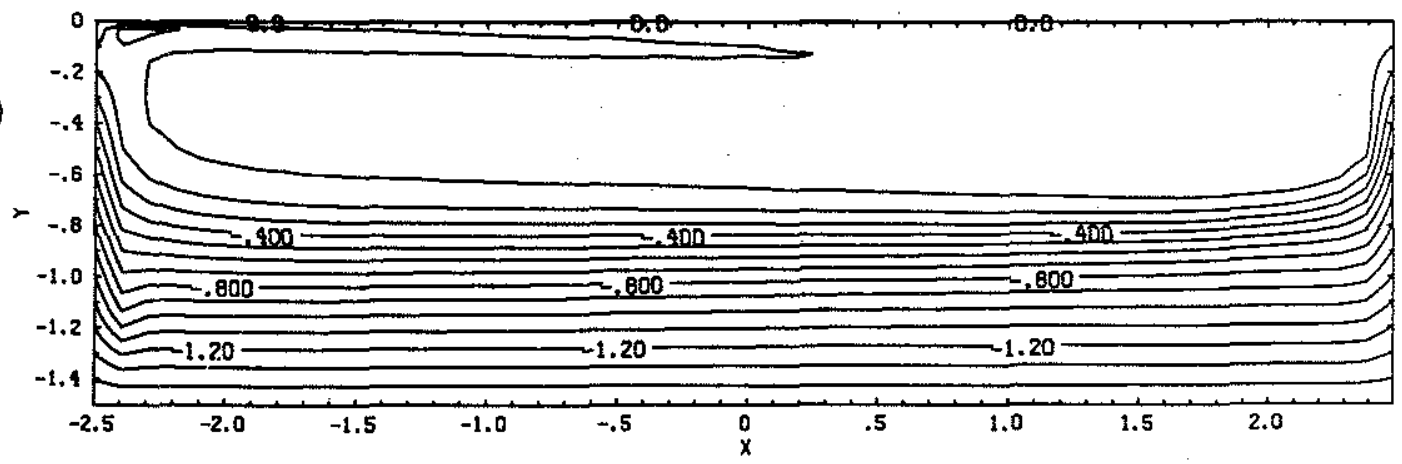

Fig. 5.1. Nondimensional streamfunction and potential vorticity fields for the steady state numerical solution. The boundary forcing is given by (5.2.6) with $Q_{n}-\beta L=$ $-\beta \sqrt{g^{\prime} H_{1}} \alpha_{1} / f_{0}$. In all the experiments the baroclinic deformation radius is $\sqrt{g^{\prime} H_{1}} / f_{0}=$ $45 \mathrm{~km}$ and the aspect ratio of the box is $\epsilon=0.3$. For this experiment $\alpha_{1}=13.33$ and $H_{2}=H_{1}$. The dissipation is $\kappa=286.2 \mathrm{~m}^{2} / \mathrm{sec}$. a) Upper layer streamfunction. Labels are multiplied by $10^{4}$. b) Upper layer potential vorticity. c) Lower layer streamfunction. Labels are multiplied by $10^{4}$. d) Lower layer potential vorticity. See Section 3 for nondimensionalization. 
(a)

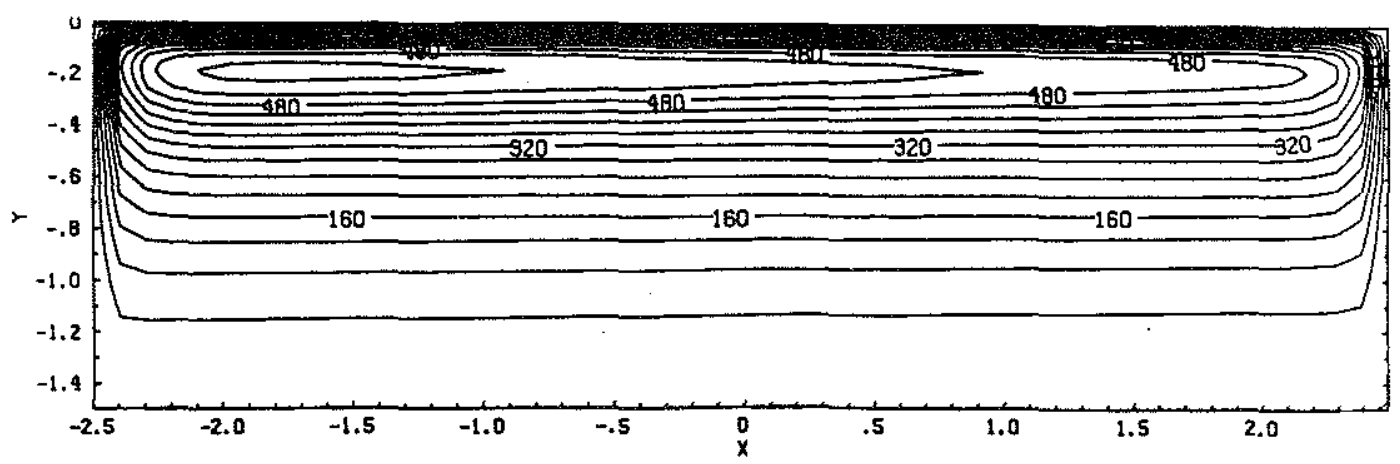

(b)

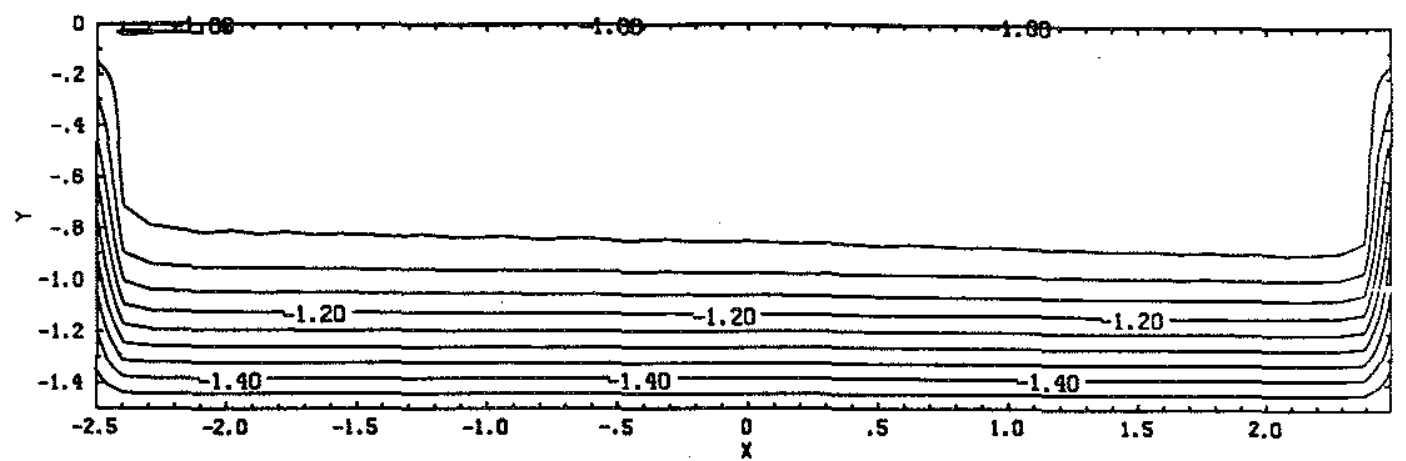

(c)

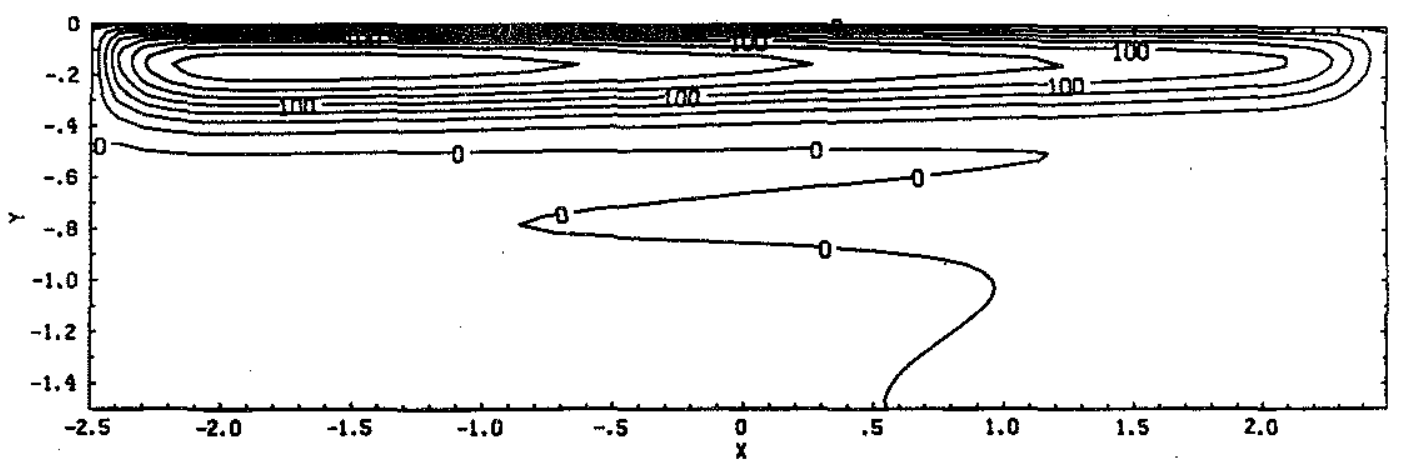

(d)

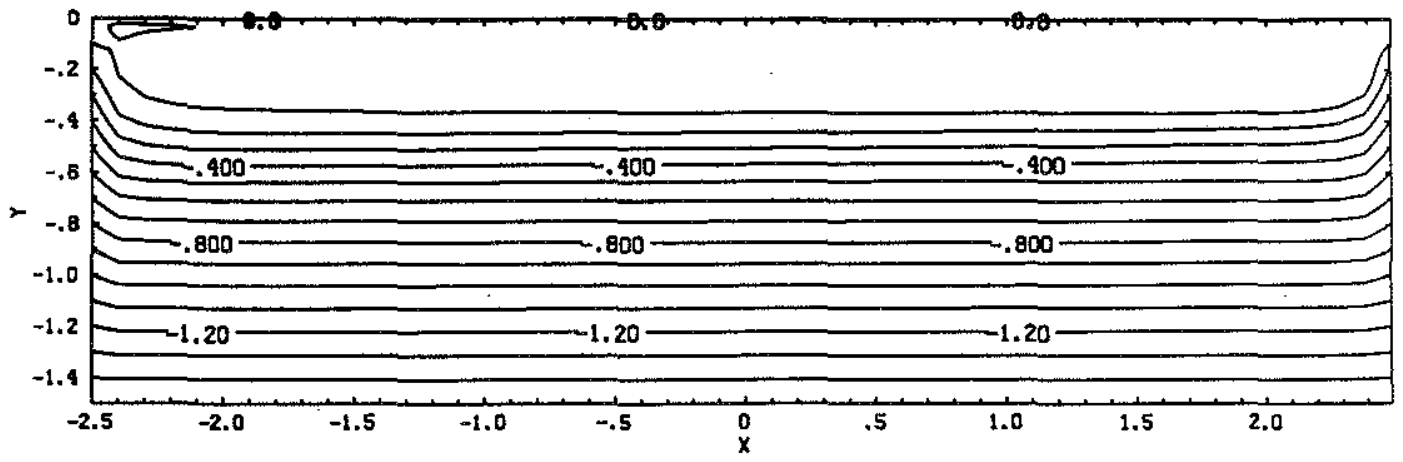

Fig. 5.2 Same as Fig. 5.1 except for $H_{2}=3 H_{1}$ and $\kappa=71.6 \mathrm{~m}^{2} / \mathrm{sec}$. The forcing has not been changed but the lower layer is deeper. a) Upper layer streamfunction. Labels are multiplied by $10^{5}$. b) Upper layer potential vorticity. c) Lower layer streamfunction. Labels are multiplied by $10^{5}$. d) Lower layer potential vorticity. Notice that the lower layer gyre has shrunk compared to that in Fig. 5.1. 
(a)

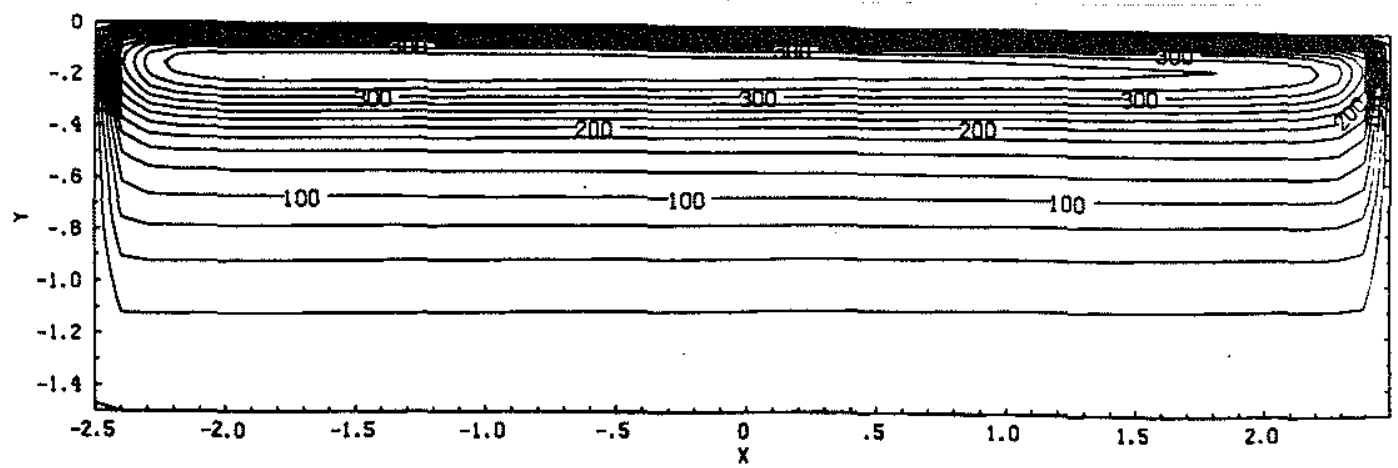

(b)

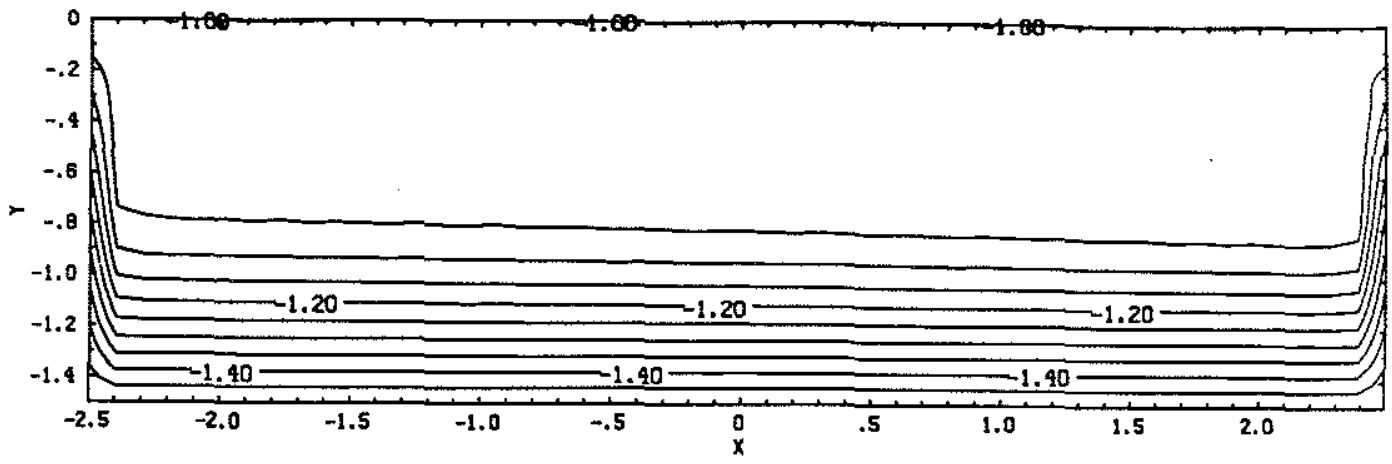

(c)

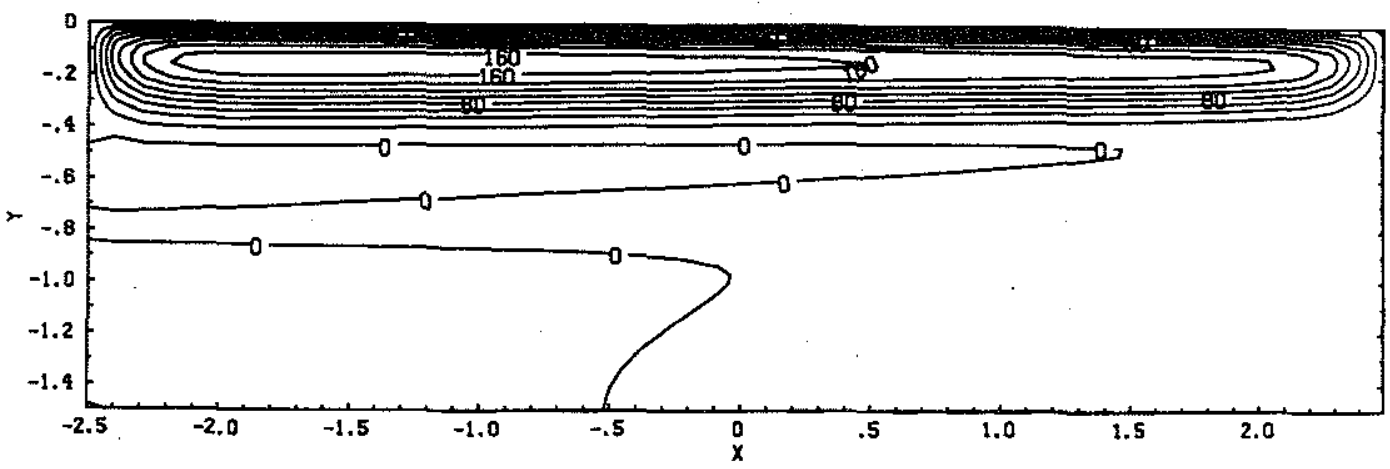

(d)

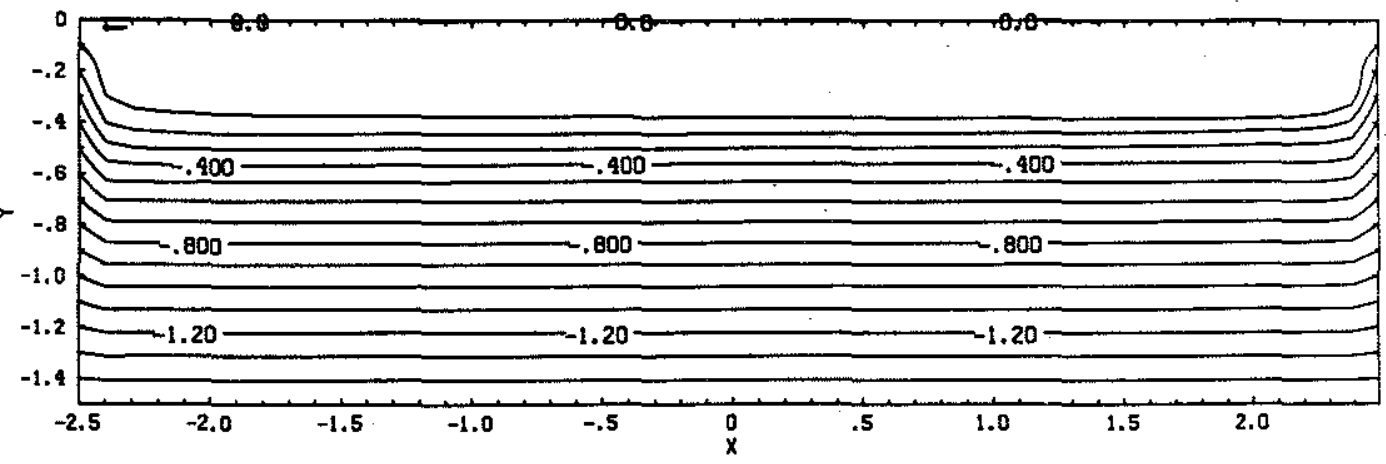

Fig. 5.3 Same as Fig. 5.1 except for $H_{2}=3 H_{1}, \alpha_{1}=18.9$ and $\kappa=101.2 \mathrm{~m}^{2} / \mathrm{sec}$. The forcing has been increased from Fig. 5.2 and the depth ratio is the same. a) Upper layer streamfunction. Labels are multiplied by $10^{5}$. b) Upper layer potential vorticity. c) Lower layer streamfunction. Labels are multiplied by $10^{5}$. d) Lower layer potential vorticity. The size of the lower layer gyre is the same as in Fig. 5.2, but its strength has increased at the expense of the upper layer flow. 
From the extremum principle (see the discussion following (5.2.4)) velocity has to be continuous everywhere, even in the presence of infinitesimal diffusion, and the tangential velocities appearing in (5.2.7) can be calculated from the interior dynamics where potential vorticity is constant. A difficulty arises because the boundaries of the gyres are unknown. For the simple choice (5.2.6), where the forcing in the upper layer is maximum at the northern boundary, unless the driving is very strong, the gyre will be pressed against the northern wall and will not fill the whole basin in the meridional direction. Therefore the southern boundaries of the gyres will be free streamlines. The situation is depicted schematically in Fig. 5.4. South of the free boundary for the upper layer gyre ( $y=L-L_{1}(x)$ in Fig. 5.4a) both layers will be at rest, except for a weak diffusively driven flow which I will neglect. Therefore on that streamline the upper layer velocity has to be zero. South of the free boundary for the lower layer ( $y=L-L_{2}(x)$ in Fig. 5.4b) no deep flow occurs, the velocities have to be continuous in both layers across the free streamline with $\vec{u}_{2}$ being zero on $y=L-L_{2}$.

This continuity is why in (5.2.7) the values of $q_{1}$ and $q_{2}$ at the rims of the gyres are the same as the potential vorticity boundary conditions (5.2.6). In fact, the northern, eastern and western boundaries of the gyres are the solid walls of the basin where potential vorticity is prescribed. The southern free boundary, where potential vorticity has an unknown value, doesn't contribute to the velocity weighted average (5.2.7).

The velocity appearing in (5.2.7) can be obtained by solving the interior problem

$$
\begin{aligned}
& \nabla^{2} \psi_{1}+F_{1}\left(\psi_{2}-\psi_{1}\right)+\beta y=\bar{q}_{1} \\
& \nabla^{2} \psi_{2}+F_{2}\left(\psi_{1}-\psi_{2}\right)+\beta y=\bar{q}_{2}
\end{aligned}
$$

with boundary conditions $\psi_{1}=\psi_{2}=0$ on $y=L, \quad x= \pm L / \epsilon, \psi_{1}=\vec{\nabla} \psi_{1}=0$ for $y \leq L-L_{1}(x)$ and $\psi_{2}=\vec{\nabla} \psi_{2}=0$ for $y \leq L-L_{2}(x)$. Notice that there is an extra pair of boundary conditions, but the widths of the gyres $L_{1}$ and $L_{2}$ are unknown and they are determined as part of the solution. It is this additional freedom which allows (5.2.8) 

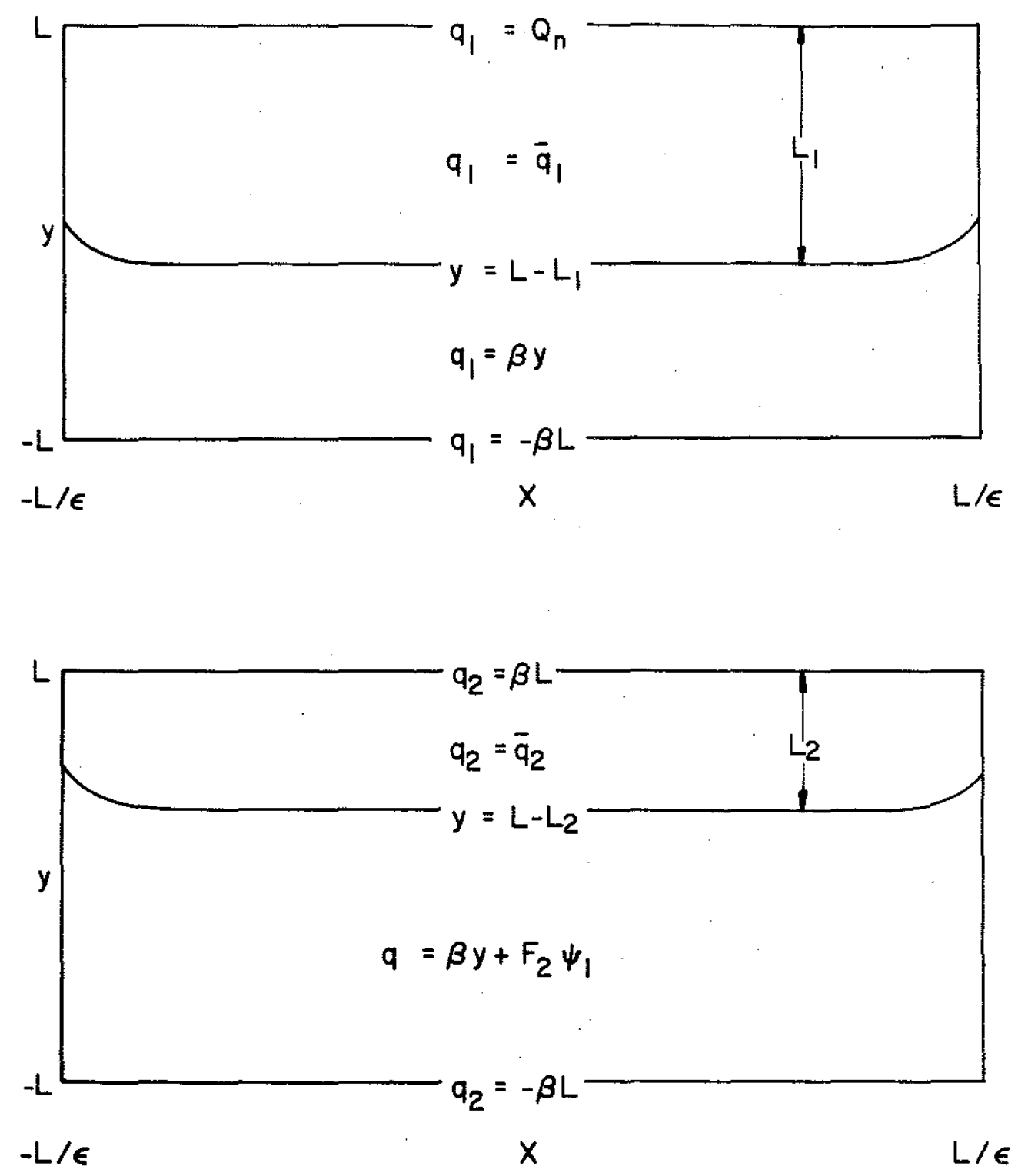

Fig. 5.4. Schematic picture of the flow regimes in the inviscid limit. a)Upper layer: south of the free streamline $y=L-L_{1}(x)$ both layers are at rest. North of $y=L-L_{1}(x)$ the surface flow circulates with uniform potential vorticity. At $y=L-L_{1}(x)$ both $\psi_{1}$ and $\vec{\nabla} \psi_{1}=0$. b) Lower layer: south of the free streamline $y=L-L_{2}(x)$ the lower layer is at rest. North of $y=L-L_{2}(x)$ the abyssal flow circulates with uniform potential vorticity. At $y=L-L_{2}(x) \psi_{1}$ and $\vec{\nabla} \psi_{1}$ are continuous and $\psi_{2}=\vec{\nabla} \psi_{2}=0$. 
to satisfy the extra boundary conditions. Although the additional boundary conditions ensure that the velocity is continuous, there will be a jump in potential vorticity across the free streamlines.

The problem becomes analytically tractable in the limit of small aspect ratio, $\epsilon$, because, away from the eastern and western walls (5.2.8) is then "one-dimensional". This approximation seems relevant to both the wind driven ERCGM's, where the recirculation appears as a long narrow gyre with aspect ratio $\epsilon \sim 0.1$, and the oceanic observations, which show meridional velocities much smaller than zonal ones. Before presenting the results of the one-dimensional approximation some general comments on (5.2.8) can be made. Forming the barotropic mode we obtain

$$
\nabla^{2}\left(H_{1} \psi_{1}+H_{2} \psi_{2}\right)+\beta y\left(H_{1}+H_{2}\right)=H_{1} \bar{q}_{1}+H_{2} \vec{q}_{2}
$$

In the barotropic equation the vortex stretching term has disappeared and the only term left to balance the planetary gradient is relative vorticity. This problem is essentially the one solved in Chapter 4. As noted in the introduction, the barotropic component of the flow represents a substantial contribution to the recirculation. Therefore, whenever the recirculation hits the bottom, a barotropic flow is established in which relative vorticity is not negligible. In this simple two-layer model the barotropic flow occurs in all the region of motion of the lower layer, so we expect relative vorticity to be important both for the eastward and the westward deep flow. This is very different from the situation depicted by $\mathrm{MN}$ who analyzed a " $\mathrm{N}$ and a half" layer model. In their work the recirculation appears as a set of baroclinic Fofonoff's (1954) gyres stacked on top of each other. The relative vorticity is confined to narrow boundary layers close to the northern, eastern and western walls and is negligible in the westward flow. This is because MN avoid the onset of a barotropic flow by always placing a resting layer below the recirculation, and consequently relative vorticity may be neglected in the interior of their gyre. 
In the presence of a deep resting layer most of the energy of the recirculating gyre is in the form of available potential energy, since the lower interface is free to deform. But the amount of possible deformation is limited. If, when the forcing is increased, the interface displacement closes potential vorticity contours in the deepest layer, then the flow will go all the way to the bottom. Once the deep layer starts moving, the growth of the interface displacement is reduced, together with the available potential energy. This is a strongly driven limit of the homogenization process envisaged by Rhines and Young (1982). Since the forcing has increased, but the increase in potential energy is limited, kinetic energy must grow, together with relative vorticity.

In the presence of substantial relative vorticity in the westward flow, the unknown gyre widths, $L_{1}$ and $L_{2}$, are determined by imposing continuity of velocity at the free streamlines, as explained in the discussion following (5.2.8). By constrast, in MN the meridional extent of the gyres is determined by requiring continuity of $\psi$ only which, when relative vorticity is neglected, implies continuity of the potential vorticity fields, while the velocities are discontinuous. The appropriate matching condition is clearly determined by what type of dissipative process is considered to act at higher order. Here it has been shown that, in the limit of infinitesimal potential vorticity diffusion, the velocities, but not potential vorticities, are continuous. The results presented in Section 4 will also indicate that, in oceanic applications where the recirculation scale is much bigger than the baroclinic deformation radius, and the recirculation strikes the bottom, the two choices give quantitatively different results. However in the absence of a barotropic flow, i.e., when the recirculation does not strike the bottom, both matching conditions give rise to approximately the same result.

The dynamical regimes are thus very different depending on whether or not the recirculation strikes the bottom of the ocean. Oceanographic observations seem to show that it does. Before proceeding with the two layer calculations I will analyze the conditions under which the recirculation hits the bottom. 


\subsection{One and a half layer model}

When the lower layer is very deep, because it is not directly forced at the boundaries, it will be at rest. As the depth $\mathrm{H}_{2}$ is reduced, the interface displacement due to the upper layer motion is eventually large enough to produce closed $q_{2}$ contours, and the abyssal waters are then set in motion. In this section I will analyze the conditions for this to occur. The critical value of $\mathrm{H}_{2}$ at which closed $q_{2}$ contours occur will be calculated, assuming that initially the lower layer is at rest.

From here after, all the analytical calculations will be done for gyres with infinitesimal aspect ratio $(\epsilon \ll 1)$. In this approximation the potential vorticity equation is one-dimensional away from the meridional walls:

$$
\psi_{1 y y}-F_{1} \psi_{1}+\beta y=\bar{q}_{1}
$$

with boundary conditions $\psi_{1}=0$ on $y=L$ and $\psi_{1}=\psi_{1 y}=0$ on $y=L-L_{1}$. From the velocity weighted average (5.2.7) the homogenized value of potential vorticity $\bar{q}_{1}$ is simply given by the value of potential vorticity on the northern boundary, $Q_{n}$, specified by (5.2.6), since the southern boundary condition doesn't contribute because the tangential velocity is zero there. The contributions from the side walls are order $\epsilon$ and can be neglected.

The solution can be found more easily if the variables are put in nondimensional form, with the choice

$$
\begin{gathered}
y=\left(L-\beta^{-1} Q_{n}\right) y^{\prime}+L \\
x=\left(L-\beta^{-1} Q_{n}\right) x^{\prime} / \epsilon \\
\psi_{n}=\beta\left(L-\beta^{-1} Q_{n}\right)^{3} \phi_{n} \\
q_{n}=\left(\beta L-Q_{n}\right) q_{n}^{\prime}+\beta L
\end{gathered}
$$

The scaling is chosen according to the results of the barotropic model, which showed that the meridional length scale is proportional to $\left(\beta L-Q_{n}\right)$, the potential vorticity 
anomaly prescribed at the northern boundary. Dropping the primes we get

$$
\phi_{1 y y}-\alpha_{1}^{2} \phi_{1}+y=-1
$$

with boundary conditions $\phi_{1}=0$ on $y=0,-\ell_{1}$ and the additional constraint $\phi_{1 y}=0$ on $y=-\ell_{1} \cdot \ell_{1}=L_{1} /\left(L-\beta^{-1} Q_{n}\right)$ is the unknown nondimensional width of the upper gyre. $\alpha_{1}=\sqrt{F_{1}}\left(L-\beta^{-1} Q_{n}\right)$ is the ratio of the forcing to the Rossby deformation radius, and is the only external parameter in this one and a half layer model.

The solution of (5.3.1) which satisfies the no normal flow conditions is

$$
\alpha_{1}^{2} \phi_{1}=y+1+\left(\ell_{1}-1\right) \cosh \alpha_{1}\left(y+\ell_{1}\right)-A \sinh \alpha_{1}\left(y+\ell_{1}\right)
$$

with $A=\left(\sinh \alpha_{1} \ell_{1}\right)^{-1}\left[1+\left(\ell_{1}-1\right) \cosh \alpha_{1} \ell_{1}\right]$.

In order to satisfy the extra condition on the continuity of tangential velocity at the free streamine $y=-\ell_{1}$, one has

$$
\ell_{1}=\frac{\lambda \cosh \lambda-\lambda}{\lambda \cosh \lambda-\sinh \lambda}
$$

where $\lambda=\sqrt{F}_{1} L_{1}=\alpha_{1} \ell_{1}$ is the ratio of the recirculation scale to the Rossby deformation radius. This is the equation which must be solved to determine $\ell_{1}$ as a function of $\alpha_{1}$.

This solution has been derived under the assumption that the lower layer is at rest. It will be valid as long as there are no closed $q_{2}$ contours. In the absence of abyssal flow $q_{2}$ is simply given by $\alpha_{1}^{2} \phi_{1} / r+y$, where $r=H_{2} / H_{1}$ is the depth ratio. If the upper layer solution just calculated gives a maximum of $q_{2}$ in the interior the extremum principle would be contradicted and this wouldn't be a possible steady state. The condition which excludes closed $q_{2}$ contours is $q_{2 y} \geq 0$ at the northern wall $y=0$. In fact an interior maximum in $q_{2}$ would require $q_{2 y}<0$ at the northern wall, since $q_{2}=0$ at $y=0$. Hence the lower layer will be motionless as long as

$$
H_{2} / H_{1} \geq r_{c}=\lambda \frac{\cosh \lambda+1}{\sinh \lambda}-2
$$




\begin{tabular}{|c|c|c|c|}
\hline$\alpha_{1}=\sqrt{F_{1}}\left(L-\beta^{-1} Q_{n}\right)$ & $\lambda=\alpha_{1} \ell_{1}$ & $\ell_{1}=L_{1} /\left(L-\beta^{-1} Q_{n}\right)$ & $r_{c}=\left(H_{2} / H_{1}\right)_{c}$ \\
\hline$\alpha_{1} \ll 1$ & $\lambda=3 \alpha_{1} / 2$ & $\ell_{1}=3 / 2$ & $r_{c}=3 \alpha_{1}^{2} / 8$ \\
0.0666 & 0.1 & 1.4997 & .0017 \\
0.1668 & 0.25 & 1.4984 & .0104 \\
0.3347 & 0.50 & 1.4938 & .0415 \\
0.6674 & 1.0 & 1.4762 & .1639 \\
1.4011 & 2.0 & 1.4274 & .6261 \\
2.2260 & 3.0 & 1.3477 & 1.314 \\
3.1148 & 4.0 & 1.2842 & 2.149 \\
4.0548 & 5.0 & 1.2331 & 3.068 \\
5.0251 & 6.0 & 1.1940 & 4.030 \\
6.0112 & 7.0 & 1.1645 & 5.013 \\
7.0046 & 8.0 & 1.1421 & 6.005 \\
8.0021 & 9.0 & 1.1247 & 7.002 \\
9.0009 & 10.0 & 1.1110 & 8.001 \\
$\alpha_{1} \gg 1$ & $\lambda=\alpha_{1}+1$ & $\ell_{1}=1+\alpha_{1}^{-1}$ & $r_{c}=\alpha_{1}-1$ \\
\hline
\end{tabular}

Table 5.1: Nondimensional gyre width and critical depth ratio as a function of the nondimensional forcing $\alpha_{1}=\sqrt{F_{1}}\left(L-\beta^{-1} Q_{n}\right)$.

Notice that this relation depends implicitly on the forcing $Q_{n}$, only through $\lambda=\alpha_{1} \ell_{1}$, i.e. the ratio of the recirculation scale $L_{1}$ to the deformation radius. The dimensional scale $L_{1}$ is proportional to the potential vorticity anomaly $\beta L-Q_{n}$ injected at the northern wall. Numerical values for $\ell_{1}$ and the critical depth ratio $r_{c}$ are presented in Table 5.1 as a function of the forcing $\alpha_{1}$. Note the first row gives the same result as the homogeneous model.

Unfortunately I am unable to directly estimate the value of the forcing $Q_{n}$ from observations. On the other hand there are good estimates for $L_{1}$ and from these one can obtain $\lambda=\sqrt{F_{1}} L_{1}=\alpha_{1} \ell_{1}$ and so infer $Q_{n}$. Typical oceanic values are $H_{1}=700 \mathrm{~m}$, $g^{\prime}=0.02 \mathrm{~m} \mathrm{sec}^{-2}, L_{1}=400 \mathrm{~km}$, which give $\lambda \simeq 11$. In this range of large values for $\lambda$ the solution to (5.3.1) is simply given by $L_{1} \simeq\left(L-\beta^{-1} Q_{n}\right)(1+1 / \lambda)$. As in the barotropic model the width of the gyre is directly proportional to the forcing. When $\lambda \gg 1(5.3 .3)$ gives $r_{c} \simeq \lambda-2$, and the critical depth ratio is very large. For this simple two layer model, the recirculation will not hit the bottom as long as the 
ratio of the lower layer to the upper layer depth is of the order of the ratio of the recirculation scale to the baroclinic radius of deformation. For the values quoted above the lower layer will be at rest only if $\mathrm{H}_{2}$ exceeds $6300 \mathrm{~m}$, for a shallower abyssal layer the recirculation will strike the bottom. The value derived by $M N$ in the one and a half layer model, neglecting relative vorticity in the westward flow, is $\ell_{1}=1$. For $\lambda \gg 1$, this is approximately the same as the value found here. However for $\lambda \gg 1$, the assumption made that no $q_{2}$ contours are closed and thus the lower layer is at rest, is not going to be met. This can be intuitively understood, by rembembering that $\lambda$ is the ratio of the recirculation scale to the baroclinic deformation radius. Since the recirculation scale is proportional to the forcing applied at the northern boundary, $\lambda$ is a measure of how stratification "resists" to the interface deformation. For large $\lambda$ such resistence is small, the interface deformation is large and the motion penetrates to the lower layer.

Typical choices for the depth of the thermocline are $H_{1}=700 \mathrm{~m}$, and the abyssal layer is then $\mathrm{H}_{2} \approx 3500 \mathrm{~m}$, the inequality (5.3.3) is not satisfied, and the assumption of a resting deep layer, made in the previous calculation and in $\mathrm{MN}$, is invalid. 


\subsection{Analytical solutions of the two layer model}

The numerical solutions of (5.2.2) shown in Figs. 5.1, 5.2 and 5.3, for small values of viscosity, are very time consuming (each run took about two hours of CRAY-1 cpu time). For this reason, and also to get more physical insight, analytical solutions were sought in the limit of long, narrow gyres. As remarked in the previous section, in this limit the homogenized value of potential vorticity, as given by the velocity weighted average (5.2.7), is independent of the velocity structure of the gyre, since the eastern and western walls give a small contribution of the order of the aspect ratio $\epsilon$, and the southern rims of the gyres have zero tangential velocity. The homogenized values of $q$ are given by the boundary value prescribed on the northern wall (see (5.2.6))

$$
\begin{aligned}
& \bar{q}_{1}=Q_{n} \\
& \bar{q}_{2}=\beta L
\end{aligned}
$$

Equations (5.2.8) are more easily solved when put in nondimensional form, with the choice made in Section 3. Dropping the primes and neglecting the longitudinal variations we get

$$
\begin{aligned}
\phi_{1 y y}+\alpha_{1}^{2}\left(\phi_{2}-\phi_{1}\right)+y & =-1 \\
\phi_{2 y y}+\alpha_{1}^{2}\left(\phi_{1}-\phi_{2}\right) / r+y & =0
\end{aligned}
$$

with boundary conditions $\phi_{1}=\phi_{2}=0$ on $y=0$ and $\phi_{1}=\phi_{1 y}=0$ on $y=-\ell_{1}$ and $\phi_{2}=\phi_{2 y}=0$ on $y=-\ell_{2} . \ell_{1}=L_{1} /\left(L-\beta^{-1} Q_{n}\right)$ and $\ell_{2}=L_{2} /\left(L-\beta^{-1} Q_{n}\right)$ are the unknown nondimensional widths of the upper and lower layer gyres respectively. $\alpha_{1}=\sqrt{F_{1}}\left(L-\beta^{-1} Q_{n}\right)$ is the ratio of the forcing to the Rossby deformation radius, $r=H_{2} / H_{1}$ is the depth ratio, and the solutions depend on these two parameters only. The solution which satisfies (5.4.2) and the boundary conditions on $y=0,-\ell_{1}$ is

$$
\begin{aligned}
& \phi_{1}=\left[1+y-\frac{\sinh \alpha_{1}\left(y+\ell_{1}\right)}{\alpha_{1}}+\left(\ell_{1}-1\right) \cosh \alpha_{1}\left(y+\ell_{1}\right)\right] / \alpha_{1}^{2} \\
& \phi_{2}=0
\end{aligned}
$$


in the region $-\ell_{1} \leq y \leq-\ell_{2}$, and

$$
\begin{aligned}
& \phi_{1}+r \phi_{2}=y\left[\frac{1+r}{6}\left(\ell_{2}^{2}-y^{2}\right)-\frac{\left(\ell_{2}+y\right)}{2}-\frac{\phi}{\ell_{2}}\right] \\
& \phi_{1}-\phi_{2}=\frac{r}{\alpha_{1}^{2}(1+r)}\left[1-\cosh \alpha y+\frac{\sinh \alpha y}{\sinh \alpha \ell_{2}}\left(1-\cosh \alpha \ell_{2}\right)\right]-\frac{\sinh \alpha y}{\sinh \alpha \ell_{2}} \phi
\end{aligned}
$$

in the region $-\ell_{2} \leq y \leq 0$, where $\alpha=\alpha_{1} \sqrt{1+r^{-1}}$.

$$
\phi \equiv\left[1-\ell_{2}-\frac{\sinh \alpha_{1}\left(\ell_{1}-\ell_{2}\right)}{\alpha_{1}}+\left(\ell_{1}-1\right) \cosh \alpha_{1}\left(\ell_{1}-\ell_{2}\right)\right] / \alpha_{1}^{2}
$$

is the value of the upper layer streamfunction at the southern boundary of the lower layer gyre $y=-\ell_{2}$.

As remarked in the previous section, for oceanographic applications, $\alpha_{1} \gg 1$, therefore the barotropic mode (5.4.4a) is of order 1 , while the baroclinic mode (5.4.4b) is $O\left(\alpha_{1}^{-2}\right)$ and thus much smaller.

The conditions that ensure that all the boundary conditions are satisfied on $y=$ $-\ell_{2}$ are:

$$
\begin{aligned}
\alpha \chi & =-\sinh \alpha \ell_{2}-\operatorname{coth} \alpha \ell_{2}\left(1-\cosh \alpha \ell_{2}-\alpha^{2} \phi\right) \\
\chi & =-\ell_{2} / 2+(1+r) \ell_{2}^{2} / 3+\phi / \ell_{2}
\end{aligned}
$$

where

$$
\chi \equiv\left[\cosh \alpha_{1}\left(\ell_{1}-\ell_{2}\right)-1-\alpha_{1}\left(\ell_{1}-1\right) \sinh \alpha_{1}\left(\ell_{1}-\ell_{2}\right)\right] / \alpha_{1}^{2}
$$

is the zonal upper layer velocity at $y=-\ell_{2}$.

The transcendental equations $(5.4 .5 \mathrm{a}, \mathrm{b})$ determine the width of the gyres $\ell_{1}$ and $\ell_{2}$. They can easily be solved numerically, and the results are shown in Fig. 5.5 for a wide range of values of $\alpha_{1}$ and depth ratios $r$.

For oceanographic applications typical values for $\alpha_{1}$ are rather large and some simplification is possible in this limit. Although the potential vorticity anomaly ( $\beta L-$ $Q_{n}$ ) is not known, the recirculation scale is going to be proportional to it, so we can assume that $L-\beta^{-1} Q_{n}$ is of the order of $L_{2}$ and check a posteriori that this is the case. For $H_{1}=900 \mathrm{~m}, g^{\prime}=1 \mathrm{~cm} \mathrm{sec}^{-2}$ and $L_{2}=300 \mathrm{~km}$ a typical value for 
$\alpha_{1}=\sqrt{F_{1}}\left(L-\beta^{-1} Q_{n}\right)$ is 10. In this range, as long as $H_{2} \geq H_{1}$ and $\left(H_{2}+H_{1}\right) / H_{1} \ll \alpha_{1}$, an approximate solution to (5.4.5) is very simple and it is given by

$$
\begin{aligned}
& \ell_{1}=1+\alpha_{1}^{-1}+O\left(e^{-\alpha_{1}}\right) \\
& \ell_{2}=3(2+2 r)^{-1}-\alpha_{1}^{-1}(1+r+\sqrt{r(1+r)})^{-1}+O\left(\alpha_{1}^{-2}\right)
\end{aligned}
$$

When the lower layer gets very deep the above approximation is not valid. The first order uniform approximation is given in Appendix A5, where it is also shown that, in order for the lower layer gyre to have a non zero width, the critical depth ratio is the same as that obtained in the one and a half layer model, eq. (5.3.3). To a first approximation the widths of the gyres are independent of $g^{\prime}$ and depend only on the ratio of the depth of the forced layer to that of the unforced one.

In the inviscid limit, the potential vorticity fields are going to be discontinuous at the southern edges of the gyres. Potential vorticity will be given by

$$
\begin{aligned}
& q_{1}^{\prime}= \begin{cases}-1 & \text { for }-\ell_{1} \leq y \leq 0 \\
y & \text { for } y<-\ell_{1}\end{cases} \\
& q_{2}^{\prime}= \begin{cases}0 & \text { for }-\ell_{2} \leq y \leq 0 \\
y+\alpha_{1}^{2} \phi_{1} / r & \text { for } y<-\ell_{2}\end{cases}
\end{aligned}
$$

When $\alpha_{1} \gg 1$, from (5.4.6), the upper layer potential vorticity field will have a weak, $O\left(\alpha_{1}^{-1}\right)$, discontinuity at the southern rim of the surface gyre, while the lower layer potential vorticity will have a discontinuity of order 1 at the southern edge of the abyssal gyre, since relative vorticity $\nabla^{2} \phi_{2}$ is discontinuous and of the order of $\ell_{2}$. Notice that also in the barotropic model potential vorticity is discontinuous at the southern edge of the gyre.

For layers of equal depth, the width of the lower layer gyre is given by $L_{2} \simeq L_{1} 3 / 4$, which is to be compared to the result obtained by MN in the two and a half layer model, $L_{2}=L_{1} / 2$. Notice that the relation between $\bar{q}_{1}$ and the width of the upper layer gyre is, to first order, the same as the one found by MN. For the oceanic range of $\alpha_{1}$, the 
(a)

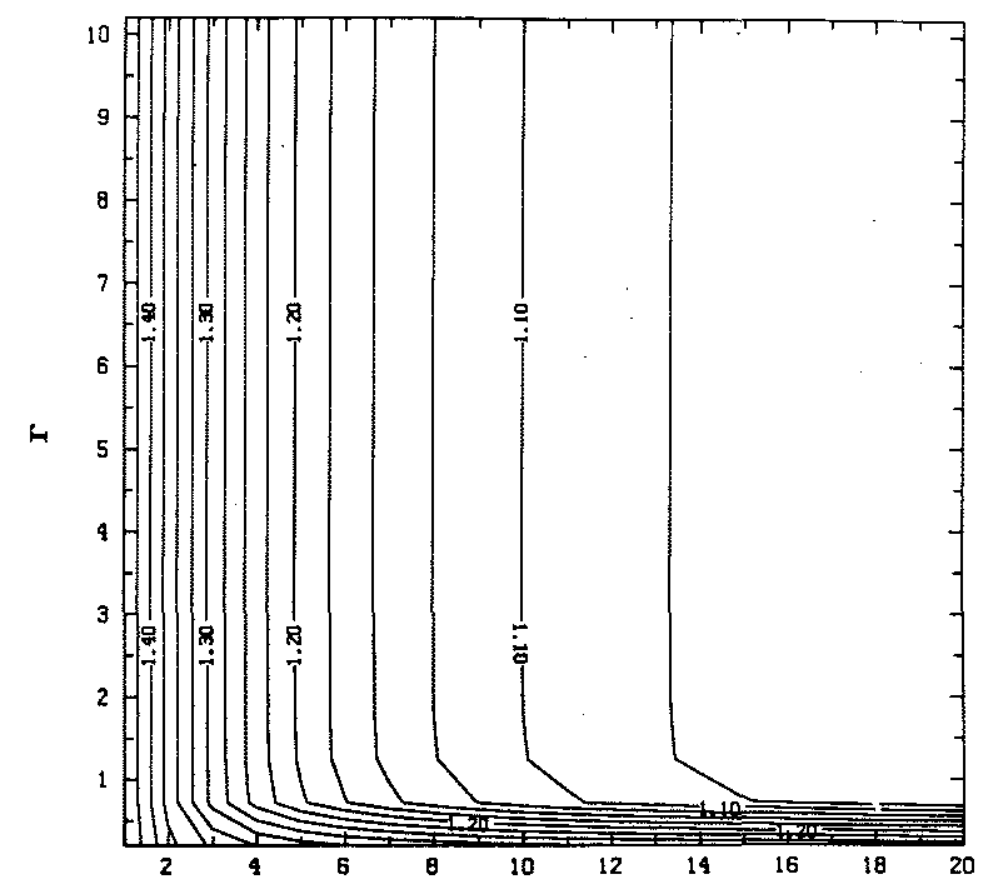

(b)

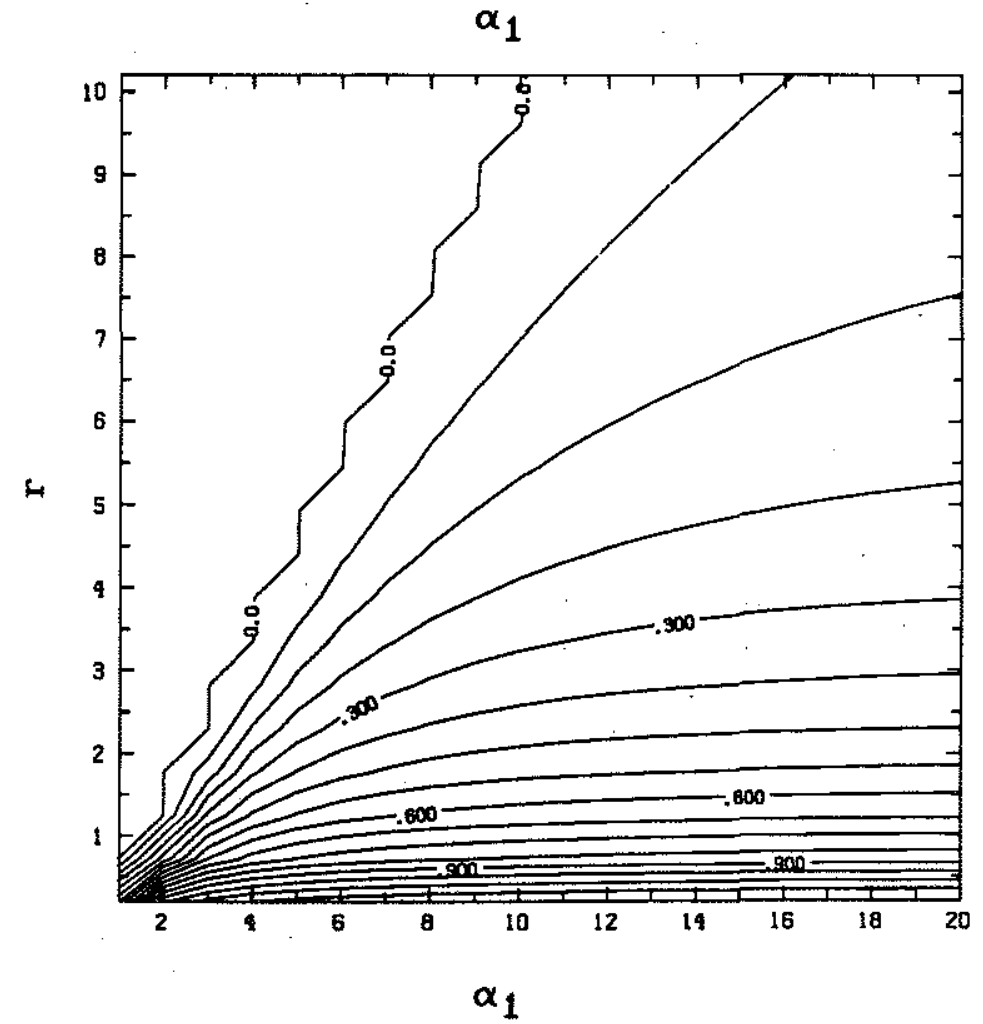

Fig. 5.5. Nondimensional gyres widths $\ell_{1}$ and $\ell_{2}$ calculated from (5.4.5) as a function of the nondimensional forcing $\alpha_{1}=\sqrt{F_{1}}\left(L-\beta^{-1} Q_{n}\right)$ and the layers depth ratio $r=$ $H_{2} / H_{1}$. a) Upper layer gyre width $\ell_{1}$. b) Lower layer gyre width $\ell_{2}$. 
barotropic transport (5.4.4a) is of order 1 while the baroclinic transport (5.4.4b) is order $\alpha_{1}^{-2}$ and thus much smaller. This could have been expected also from the general results presented in Section 2. The steady state form of the energy equation (5.2.5) in nondimensional form is

$$
\begin{gathered}
\oint\left(q_{1 b}^{\prime}-y\right) \vec{u}_{1}^{\prime} \cdot d \vec{\ell}=\int d A\left[\left(\nabla^{2} \phi_{1}\right)^{2}+r\left(\nabla^{2} \phi_{2}\right)^{2}+\alpha_{1}^{2}\left(\vec{u}_{1}^{\prime}-\vec{u}_{2}^{\prime}\right)^{2}\right] \\
\text { where } \quad q_{1 b}^{\prime}=\frac{q_{b}(s)-\beta L}{\beta L-Q_{n}} .
\end{gathered}
$$

From the "extremum principle" (5.2.4) nondimensional relative vorticity has to be of order 1. Thus the square of the vertical shear $\alpha_{1}^{2}\left(\vec{u}_{1}^{\prime}-\vec{u}_{2}^{\prime}\right)^{2}$ is at most of order 1 and if $\alpha_{1}$ is big the vertical shear will be small, no larger than order $\alpha_{1}^{-1}$.

A remarkable property of the barotropic part of the flow (5.4.4a) is that in the limit of large $\alpha_{1}$ it is independent of $\alpha_{1}$. In this limit the maximum barotropic transport occurs (to first order) at $y=-2 \ell_{2} / 3$. In the barotropic model, the latitude of maximum transport was $y=-2 \ell / 3$ where $\ell$ was the width of the gyre, and the total nondimensional transport was given by $\phi_{\max }=2 \ell^{3} / 81$. In the present model it's

$$
H^{-1}\left(H_{1} \phi_{1}+H_{2} \phi_{2}\right)_{\max }=\frac{2}{81} \ell_{2}^{3}+O\left(\alpha_{1}^{-2}\right)
$$

To a first approximation the transport carried by the barotropic flow in the present baroclinic model is the same as that obtained with a homogeneous model, if the width of the deep gyre, $\ell_{2}$, is considered as representative of the width of the whole recirculation, and the transport is independent of the vertical distribution of the flow.

This is why the inertial gyre has a weakly depth dependent structure: the barotropic国 "core" is responsible for the bulk of the transport. Outside the barotropic core there is a weak "baroclinic fringe" with small velocities of $O\left(\alpha_{1}^{-1}\right)$. Essentially, it is this part of the flow that $\mathrm{MN}$ analyzed in their two and a half layer model. Observations, on the other hand, suggest that, in the North Atlantic, most of the transport resides in the 
barotropic core, and this is certainly the case in ERCGM's (e.g. Schmitz and Holland (1986)).

In Fig. 5.6 the streamfunction and zonal velocity fields are plotted for $\alpha_{1}=13.33$ and $H_{1}=H_{2}$. This corresponds to the numerical solution shown in Fig. 5.1. For these values the non-dimensional widths of the gyres are $\ell_{1}=1.08$ and $\ell_{2}=0.72$. Notice that the maximum of the streamfunction occurs at the same latitude in both layers and the transport is almost equally distributed between the two layers. Similarly, the maximum westward velocity is equal in both layers and is located at the same latitudes. The maximum eastward velocity is located at the northern edge of the gyres, but, unlike the westward flow, the surface value is about twice as large as the deep one.

The dynamical balance obtained when the recirculation goes all the way to the bottom and establishes a barotropic "core" is opposite to that envisaged by MN. In their model, baroclinicity was dominant in the center of the recirculation and relative vorticity was confined to the northern wall. Here baroclinic velocities are confined to the edge of the gyres, in boundary layers of the order of the Rossby deformation radius, while the center is dominated by a depth independent flow in which relative vorticity is important. The reason why depth dependence is confined to the outer edges of the gyre is clear. The forcing, which varies with depth, is applied at the boundaries of the gyres. The baroclinic signature impressed at the boundaries can only penetrate inwards a distance of the order of the baroclinic radius of deformation. On the other hand, once a barotropic flow is established, the only length scale which enters in the dynamics of the vertically integrated flow is $\left(L-\beta^{-1} Q_{n}\right)$, which is much larger than the baroclinic deformation radius.

For the case shown in Fig. 5.6 the dimensional scales of the gyres, as given by the analytical solution, are $L_{1}=644 \mathrm{~km}$ and $L_{2}=429 \mathrm{~km}$. To obtain these values I chose the following parameters: $g^{\prime}=1 \mathrm{~cm} \mathrm{sec}^{-2}, \quad H_{1}=H_{2}=2000 \mathrm{~m}$. The total transport is $183.6 \mathrm{~Sv}$, of which $156 \mathrm{~Sv}$ can be calculated from (5.4.8), which is a depth independent 
(a)
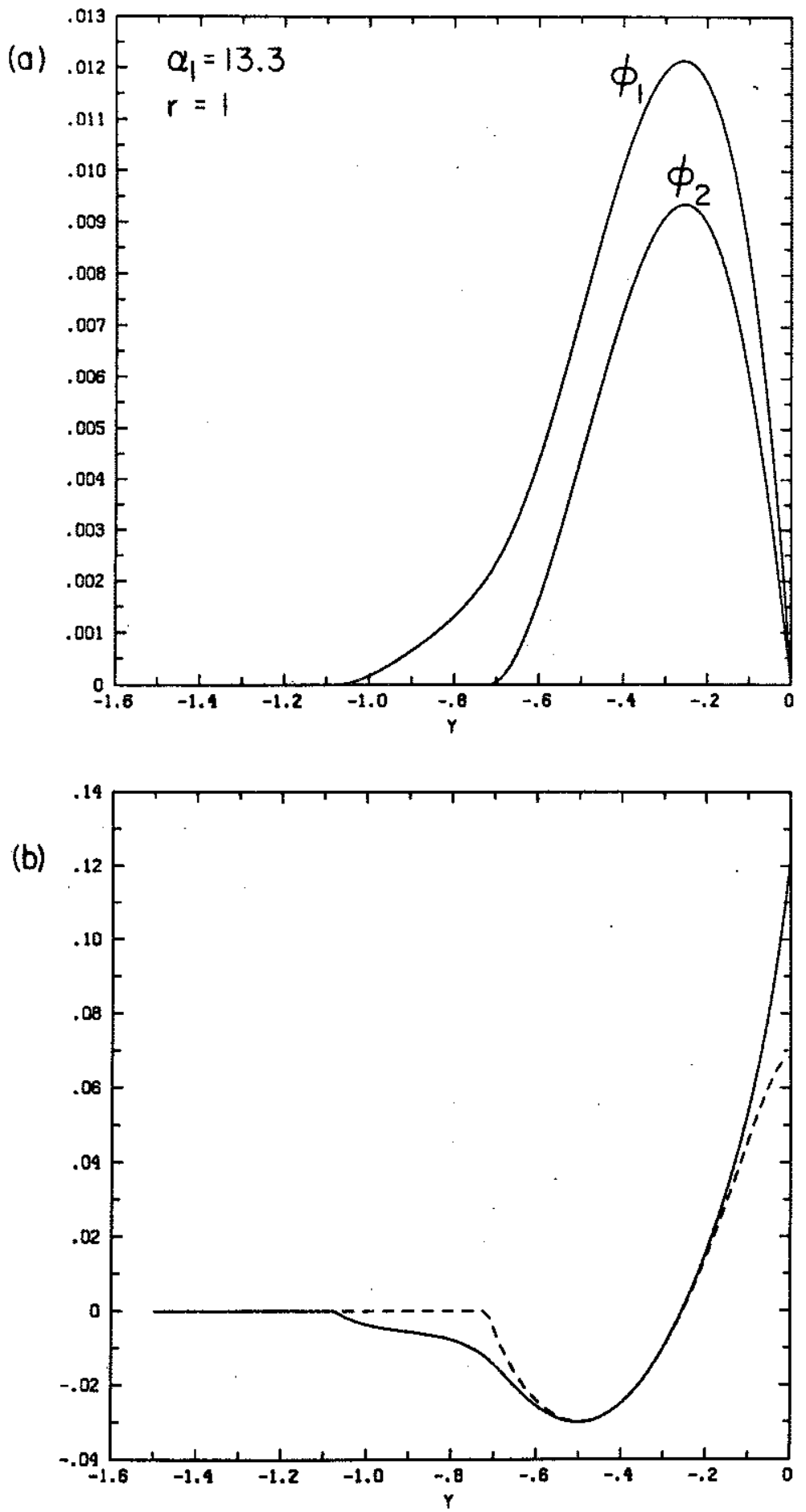

Fig. 5.6. Nondimensional streamfunction and zonal velocity as a function of latitude calculated from (5.4.2), for $\alpha_{1}=13.33$ and $r=1$. For this choice of the parameters the nondimensional gyre widths are $\ell_{1}=1.08, \ell_{2}=0.72$. a) Streamfunctions. b) Zonal velocity: solid line corresponds to the upper layer, the dashed line to the lower layer. 
approximation. The dimensional maximum westward velocity is $28.4 \mathrm{~cm} / \mathrm{sec}$, both at the surface and at depth, while the maximum eastward velocity is $113.8 \mathrm{~cm} / \mathrm{sec}$ at the surface and $66.4 \mathrm{~cm} / \mathrm{sec}$ at depth. These values are big because the forcing is very strong (remember that the upper forced layer is $2000 \mathrm{~m}$ deep).

When the upper layer is shallower and the lower layer is deeper the picture appears much more realistic. In Fig. 5.7 the streamfunction and velocity fields are plotted as a function of latitude for $\alpha_{1}=13.3$ and $H_{2}=3 H_{1}$. This corresponds to the numerical solution shown in Fig. 5.2. The gyre in the lower layer has considerably shrunk and its width is about a third of that in the upper layer gyre one. The streamfunction maximum is reduced in both layers with respect to the case shown in Fig. 5.6 where $H_{1}$ was equal to $H_{2}$. Notice, however, that the maximum westward velocity is still almost equal in the two layers, while the maximum eastward velocity in the upper layer is about 7.5 times that in the lower layer. For $H_{1}=1000 \mathrm{~m}, g^{\prime}=0.02 \mathrm{~m} / \mathrm{sec}^{2}$, the total transport is 28.6 Sv of which $9.5 \mathrm{~Sv}$ are carried by the lower layer and 16.5 are depth independent. In the abyssal layer the maximum westward velocity is $3.6 \mathrm{~cm} / \mathrm{sec}$, and the maximum eastward velocity is $7.2 \mathrm{~cm} / \mathrm{sec}$. The width of the deep recirculating gyre, $L_{2}$, is $203 \mathrm{~km}$ of which $125 \mathrm{~km}$ are in the region of westward flow. Notice that because the barotropic core has a width of $\approx 0.3$ the depth independent transport computed from $(5.4 .8)$ is comparable to the transport carried by the "baroclinic fringe" which is $O\left(\alpha_{1}^{-2}\right)$.

If the forcing applied at the northern wall, $\beta L-Q_{n}$, is increased keeping the depth and density ratio fixed, the flow becomes increasingly depth independent. In Fig. 5.8 the streamfunction and velocity fields are presented as a function of latitude, for $\alpha_{1}=18.9$ and $H_{2}=3 H_{1}$. Although the ratio of the gyres widths is practically unchanged relative to that in Fig. 5.7, the flow is more vertically coherent. Now the total transport is $72 \mathrm{~Sv}$ half of which is carried by the abyssal layer. Of this flow, about $70 \%$ can be accounted for by (5.4.8). The maximum of the westward jet occurs at the same latitude, is equal in both layers and has an amplitude of $9.2 \mathrm{~cm} / \mathrm{sec}$. The 

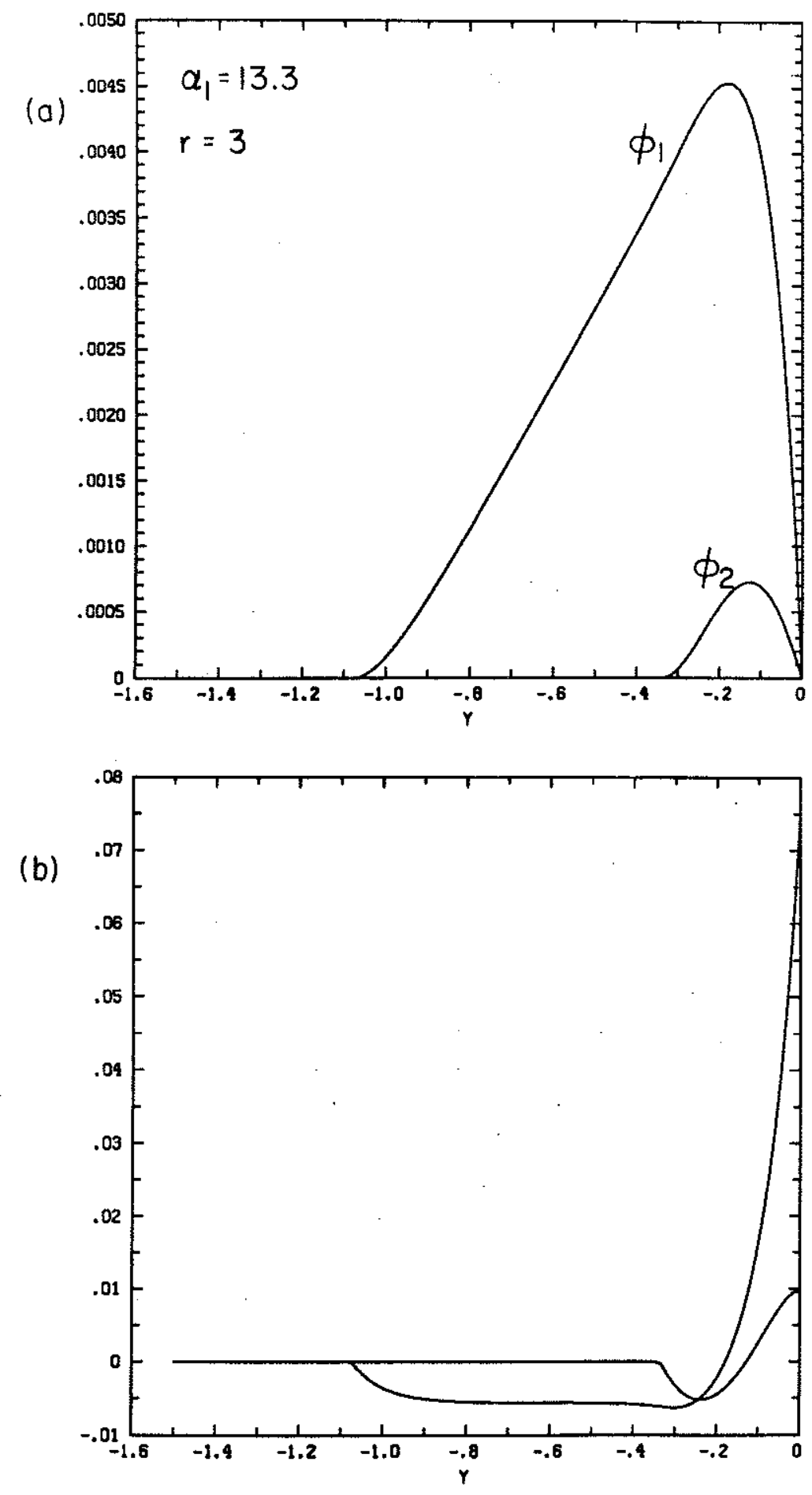

Fig. 5.7. Same as Fig. 5.6 except for $r=3$. For this choice of the parameters the nondimensional gyre widths are $\ell_{1}=1.08, \ell_{2}=0.34$. a) Streamfunctions. b) Zonal velocity $\left(u_{1}\right.$ is easily recognized by its larger values compared to $\left.u_{2}\right)$. The abyssal gyre has shrunk compared to Fig. 5.6 and the transport has decreased. 
abyssal recirculating gyre is $295 \mathrm{~km}$ wide, while the surface gyre is three times as large. Notice, however, that the surface layer velocities in the large region where the abyssal layer is at rest, are weaker than the velocities in the barotropic core. Therefore, in the baroclinic fringe, the neglected diffusive effects and the Sverdrup interior flow may become comparable to the westward surface flow.

\subsection{Numerical results}

Steady solutions of (5.2.2), with potential vorticity boundary conditions (5.2.6), were found by time integration from rest, with the use of a quasigeostrophic two layer numerical model developed by Dr. Ierley. Solutions were considered to have reached the state state when (see (5.2.3))

$$
\oint_{b a s i n} \vec{\nabla} q_{n} \cdot \hat{n} d \ell \leq 10^{-2}
$$

with the integral performed along the boundaries of the box.

The motivations for seeking numerical solutions after having found simple analytical approximations were several.

1) Verify that the analytical solutions described in Section 5.4 are indeed the inviscid limit of the full viscous problem.

2) Explore the effects that make the problem expressed by (5.2.2) analytically intractable, such as finite, although small viscosity $\kappa$ and aspect ratio $\epsilon$.

In Fig. 5.9a,b the potential vorticity and zonal velocity fields at the longitude of maximum transport $(x=0)$ are plotted as a function of latitude. This corresponds to the case shown in Fig. 5.1, with $\alpha_{1}=\sqrt{F_{1}}\left(L-\beta^{-1} Q_{n}\right)=13.33, H_{1}=H_{2}$. In all the experiments the aspect ratio $\epsilon$ of the box containing the flow is 0.3 . In this experiment the effective aspect ratio of the gyres is $\simeq 0.15$, since the bulk of the flow occupies only the northern half of the basin. The Reynolds number, defined as $\psi_{2 \max } / \kappa$ is $\simeq 207$. In Fig. 5.9a the velocity fields of the numerical and analytical solutions are shown. 

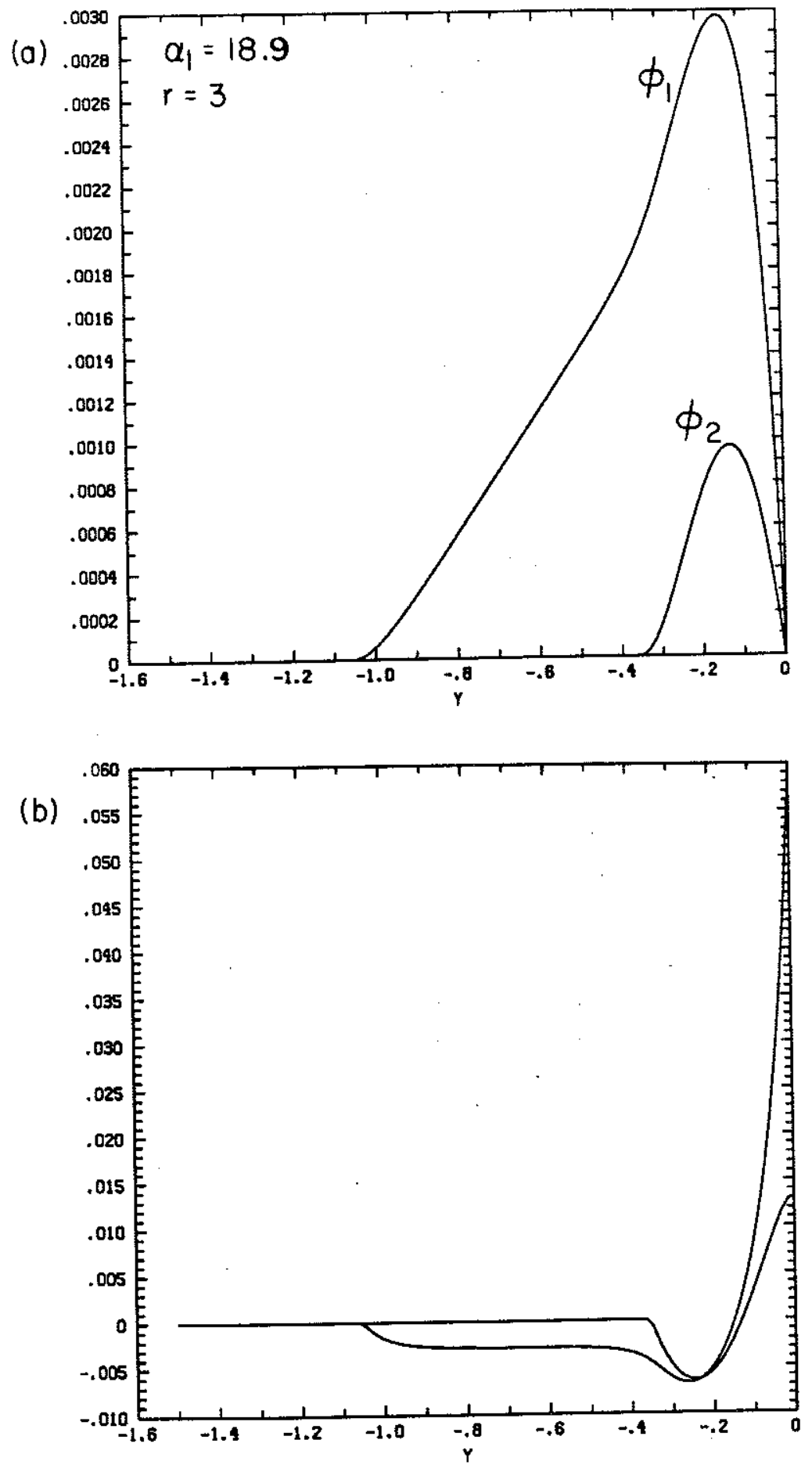

Fig. 5.8. Same as in Fig. 5.6 except for $\alpha_{1}=18.9$ and $r=3$. For this choice of the parameters the nondimensional gyre widths are $\ell_{1}=1.05, \ell_{2}=0.35$. a) Streamfunctions. Notice that $\phi_{1} \simeq \phi_{2} H_{2} / H_{1}$. b) Zonal velocity ( $u_{1}$ is easily recognized by its larger values compared to $u_{2}$ ). The forcing has been increased with respect to Fig. 5.7 while the depth ratio is the same. The gyres widths are practically unchanged but the transport is increased in the lower layer and decreased in the upper layer. 
The solid and long-dashed lines are the analytic solutions for the upper and lower layer respectively. The dot-dashed and short-dashed lines are the numerical solutions for the upper and lower layer respectively. Although the actual values are in good but not excellent agreement, the structure of the numerical solution is identical to that of the analytic solution. The pair resulting from the numerical experiment has a slight shift in amplitude and position and scale relative to the analytical pair. The same structural similarity persists if viscosity is increased. Fig. 5.9c shows the velocity fields for the same case with viscosity increased by a factor of 6 from the case shown in Fig. 5.9a. The vertical shear of the maximum eastward velocity $\left(u_{1}^{\prime}-\left.u_{2}^{\prime}\right|_{y=0}\right)$ is exactly the same as in the analytical calculations, regardless of the size of the dissipation, and the same applies for the vertical shear at the maximum westward velocity.

Notice that friction enhances the vertical coherence of the flow. In the most viscous experiment (Fig. 5.9c) the confinement of the baroclinic signature to the northern portion of the flow is even more pronounced than in the analytical calculation and the baroclinic "fringe" south of the barotropic core is completely erased.

In Fig. 5.9d the potential vorticity field for the latter numerical experiment is shown. The analytical prediction is given in (5.4.7). Remember that in (5.4.7) the upper layer potential vorticity has a weak $O\left(\alpha_{1}^{-1}\right)$ discontinuity at the southern edge of the surface gyre, while in the lower layer the discontinuity is of $O(1)$. In the viscous experiment there is no sign of discontinuity at the southern edge of the abyssal gyre, but if Fig. $5.9 \mathrm{~d}$ is compared with Fig. 5.9b a tendency toward a discontinuity can be seen. Although the homogenized value of $q_{2}^{\prime}$ has gone up relative to the more viscous run, thus approaching the theoretical prediction, the value of $q_{2}^{\prime}$ south of the gyre has decreased, approaching $y+\alpha_{1}^{2} \phi_{1} / r$ (dashed curve).

In Fig. 5.10a,b the velocity and potential vorticity fields from the numerical experiment shown in Fig. 5.2 at the longitude of maximum transport $(x=-0.87)$ are plotted as a function of latitude. For this run $\alpha_{1}=13.33, H_{2}=3 H_{1}$ and the Reynolds 

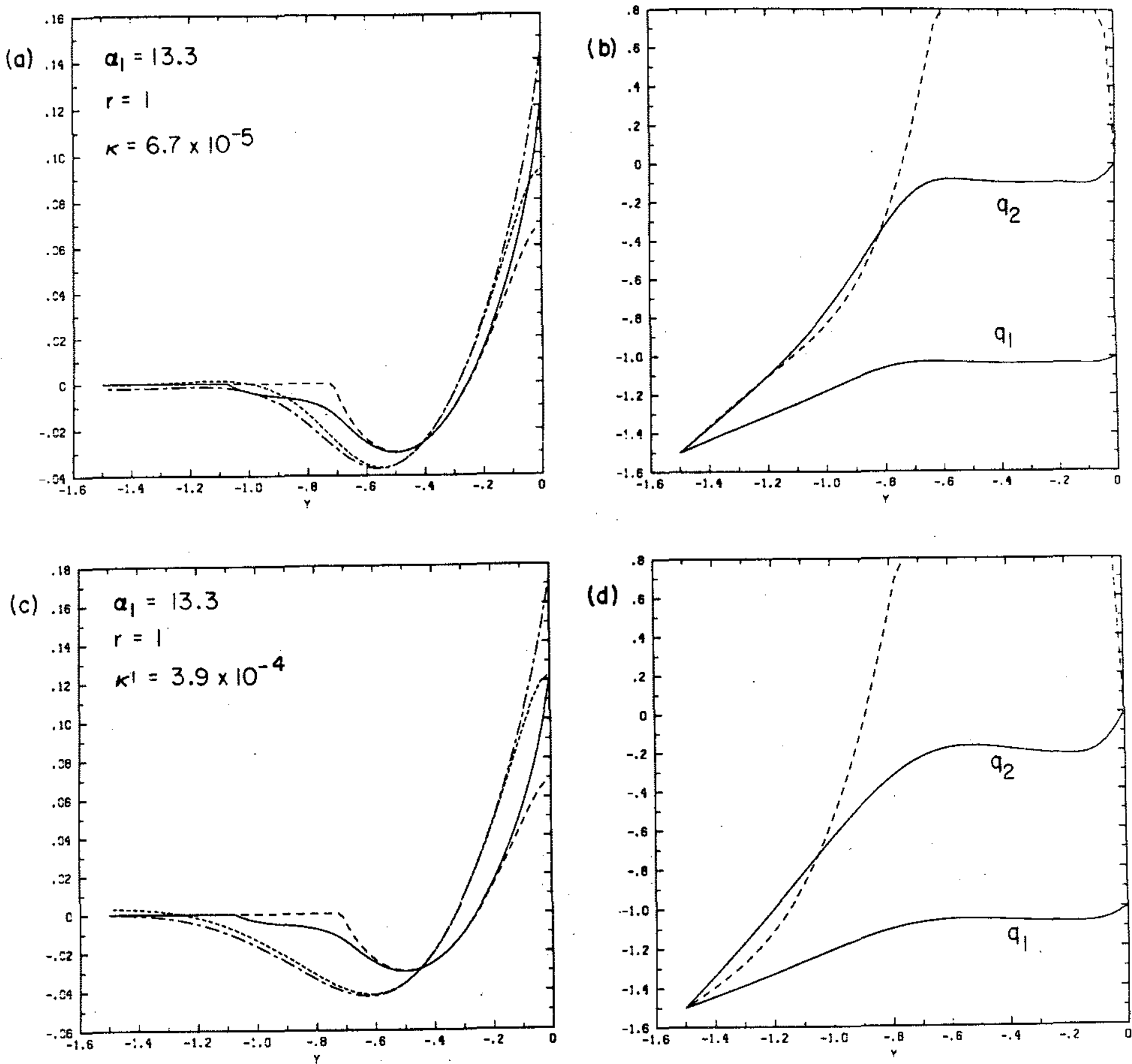

Fig. 5.9. Meridional section of the zonal velocity and potential vorticity fields at the longitude of maximum transports for the numerical run shown in Fig. 5.1. $\alpha_{1}=$ 13.33, $r=1$. a) Zonal velocity fields at $x=0$ for $\kappa=286.2 \mathrm{~m}^{2} / \mathrm{sec}$, compared with the analytic solution shown in Fig. 5.6b. Dashed-dotted line: numerical upper layer velocity. Solid line:analytic upper layer velocity. Short dashed line: numerical lower layer velocity. Long dotted line: analytic lower layer velocity. b) Potential vorticity fields at $x=0$ for $\kappa=286.2 \mathrm{~m}^{2} / \mathrm{sec}$. The dashed line is $q_{2}^{\prime}=y+\alpha_{1}^{2} \phi_{1} / r$. c) Same as Fig. $5.9 \mathrm{a}$ except for $\kappa=1,717.3 \mathrm{~m}^{2} / \mathrm{sec}$. The longitude of maximum transport is at $x=-1.27$. d) Same as Fig. 5.9b except for $\kappa=1,717.3 \mathrm{~m}^{2} / \mathrm{sec}$. The longitude of maximum transport is at $x=-1.27$. 
number is 103. Again the structure of the flow is the same as that predicted by the theory, and in the barotropic core the values are in excellent agreement with the theory, especially in the upper layer where the flow is more vigorous. Fig. 5.10c,d show the same experiment for a value of viscosity 4 times larger than that in Fig. 5.10a,b. In this experiment viscosity is less efficient at erasing the vertical shear in the westward portion of the baroclinic fringe than it was for layers of equal depths. The reason for this is quite clear. Except for a small region close to the edge of the upper layer gyre, from the analytical solution we can see that in most of the southern fringe the vertical shear is practically independent of latitude. In the absence of horizontal gradients interfacial friction is not very effective at locking the two layers together, although it does increase the lower layer velocity near the edge of the abyssal gyre, extending the region of vertical coherence.

The potential vorticity fields are shown in Figs. 10b,d. For the more inviscid run $q_{n}^{\prime}$ approaches the profile predicted by the theory, and a smooth boundary layer begins to be visible at the southern edge of the abyssal gyre.

Finally in Fig 11 a meridional section along the longitude of maximum transport $(x=-0.87)$ of the zonal velocity and potential vorticity fields is plotted for the experiments shown in Fig. 5.3. This corresponds to the case $\alpha_{1}=18.9, H_{2}=3 H_{1}$. For this experiment the Reynolds number is 200 . Both the potential vorticity and the velocity are in excellent agreement with the theory, even in the baroclinic fringe, supporting the result that, in the inviscid limit, the velocities have to be continuous, but not the potential vorticity. In Fig. 5.12a,b I have plotted the relative vorticity, $\nabla^{2} \phi_{2}$, and the vortex stretching term, $\alpha_{1}^{2}\left(\phi_{1}-\phi_{2}\right) / r$, of the lower layer for the run shown in Fig. 5.3. In the barotropic core region $(y>-0.4)$ relative vorticity is as large as the vortex stretching, and in fact its values in the center of the gyre are larger than the values at the edges. Notice also that the vortex stretching term is constant at the center, and 

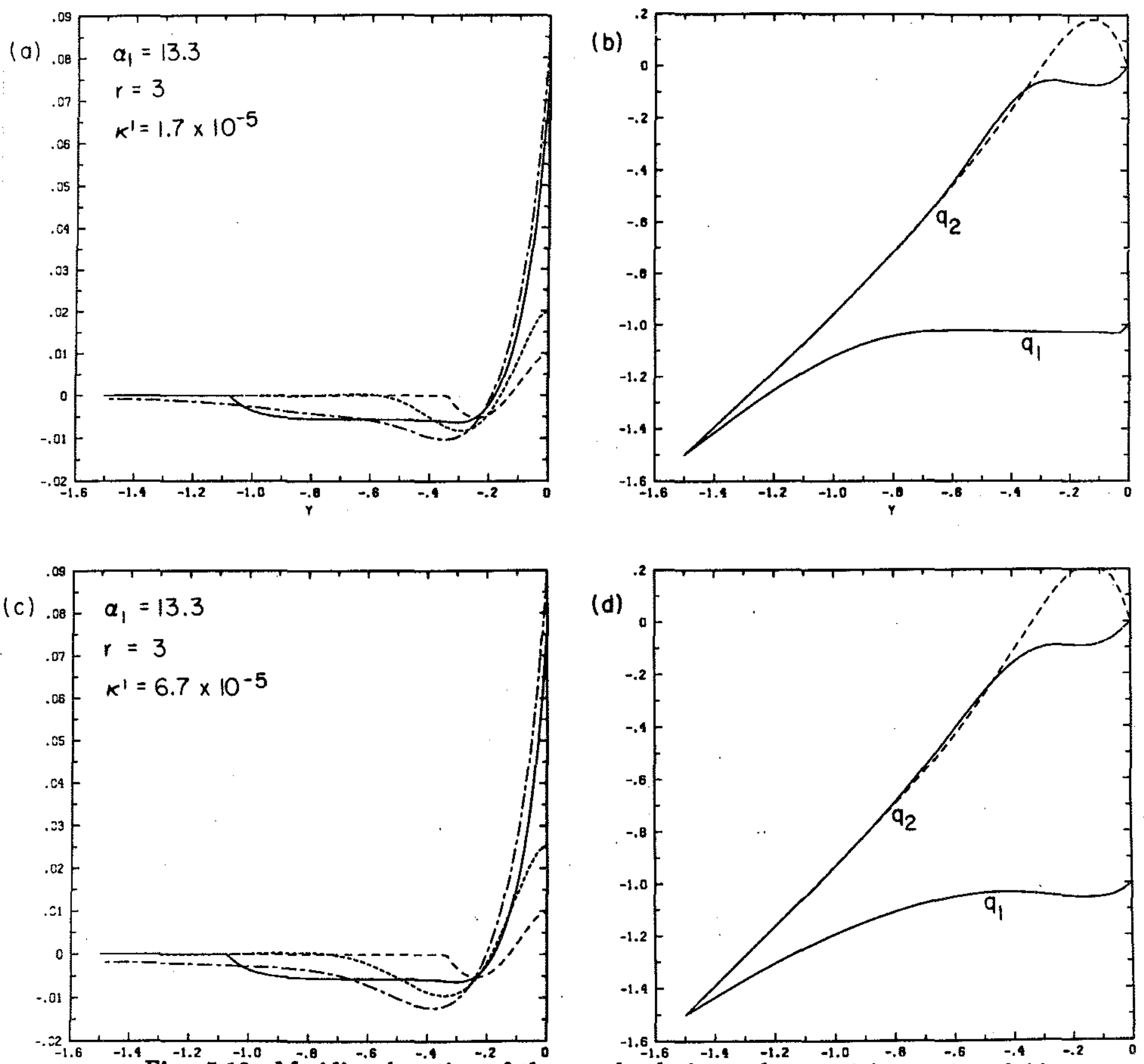

Fig. 5.10. Meridional section of the zonal velocity and potential vorticity fields at the longitude of maximum transports for the numerical run shown in Fig.5.2. $\alpha_{1}=$ 13.33, $r=3$. a) Zonal velocity fields at $x=-0.87$ for $\kappa=71.6 \mathrm{~m}^{2} / \mathrm{sec}$, compared with the analytic solution shown in Fig. 5.7b. Dashed-dotted line: numerical upper layer velocity. Solid line:analytic upper layer velocity. Short dashed line: numerical lower layer velocity. Long dotted line: analytic lower layer velocity. b) Potential vorticity fields at $x=-0.87$ for $\kappa=71.6 \mathrm{~m}^{2} / \mathrm{sec}$. The dashed line is $q_{2}^{\prime}=y+\alpha_{1}^{2} \phi_{1} / r$. c) Same as Fig. 5.10a except for $\kappa=286.3 \mathrm{~m}^{2} / \mathrm{sec}$. d) Same as Fig. 5.10b except for $\kappa=286.2 \mathrm{~m}^{2} / \mathrm{sec}$. 

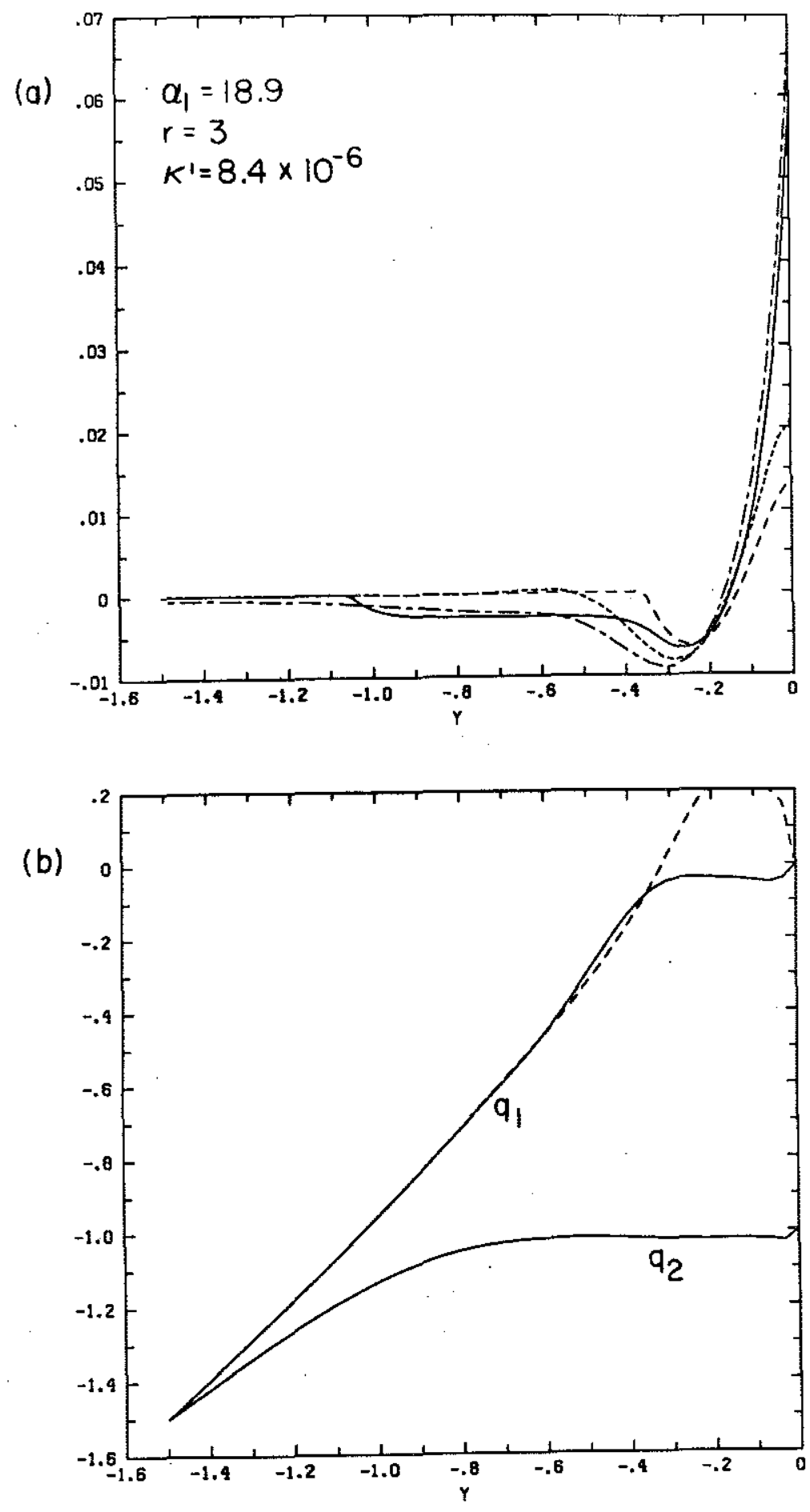

Fig. 5.11. Meridional section of the zonal velocity and potential vorticity fields at the longitude of maximum transports for the numerical run shown in Fig. 5.3. $\alpha_{1}=$ $18.9, r=3$. a) Zonal velocity fields at $x=-0.87$ for $\kappa=101.2 \mathrm{~m}^{2} / \mathrm{sec}$, compared with the analytic solution shown in Fig. 5.8b. Dashed-dotted line: numerical upper layer velocity. Solid line:analytic upper layer velocity. Short dashed line: numerical lower layer velocity. Long dotted line: analytic lower layer velocity. b) Potential vorticity fields at $x=-0.87$ for $\kappa=101.2 \mathrm{~m}^{2} / \mathrm{sec}$. The dashed line is $q_{2}^{\prime}=y+\alpha_{1}^{2} \phi_{1} / r$. 
(a)
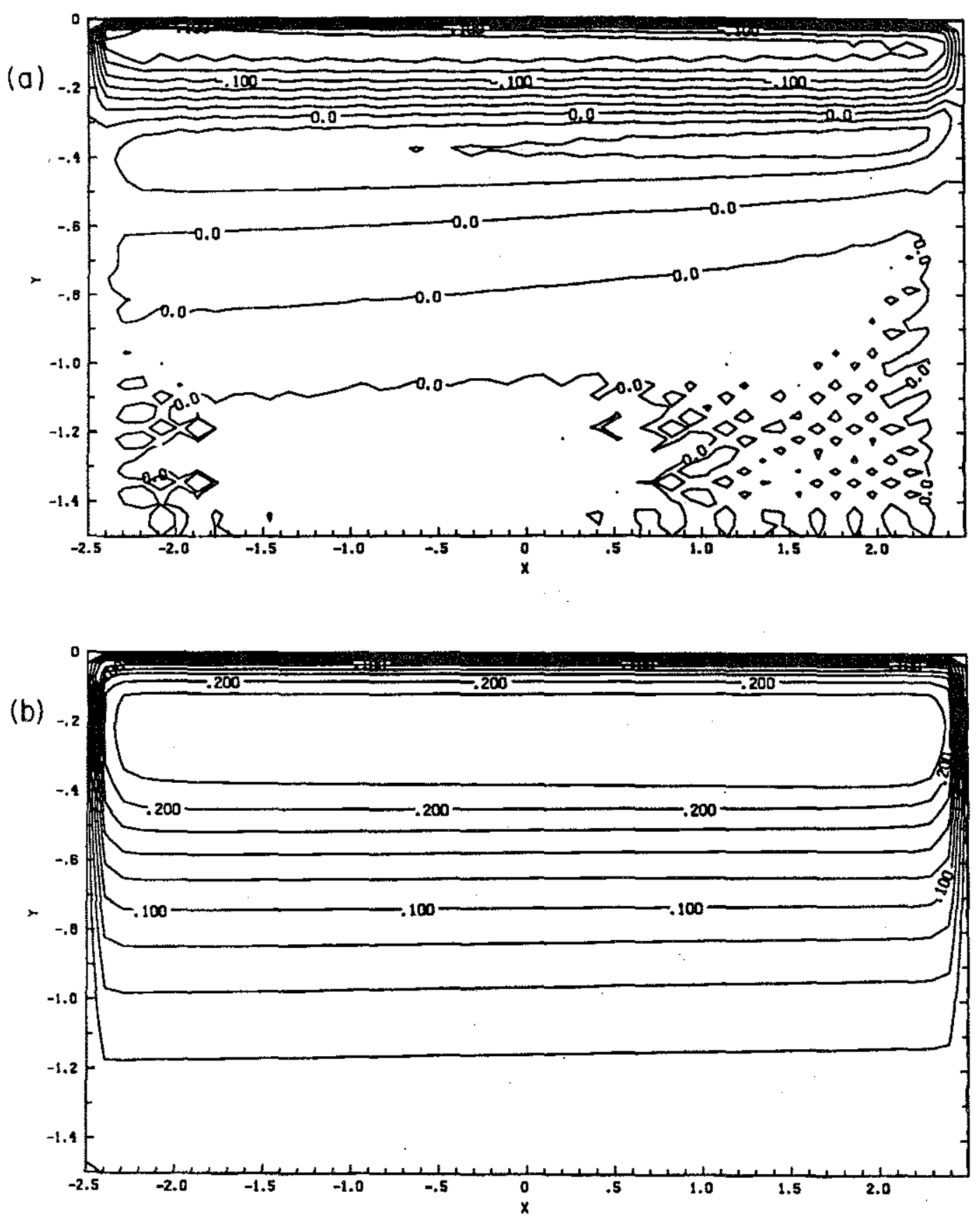

Fig. 5.12. Lower layer relative vorticity and vortex stretching term for the numerical experiment shown in Fig. 5.3. a) Relative vorticity $\nabla^{2} \phi_{2}$. b) Vortex stretching $\alpha_{1}^{2}\left(\phi_{1}-\right.$ $\left.\phi_{2}\right) / r$. The meridional scale has been expanded by a factor of three. The southern boundary of the abyssal gyre is at $y \sim-0.4$. 
there the planetary vorticity gradient is balanced by the relative vorticity. The numerical results confirm the structure suggested by the analytic solution: baroclinicity is confined to the edge of the recirculation while the center is dominated by inertial effects.

\subsection{Summary and conclusions}

Some of the prominent features of the inertial gyre observed in the wind driven ERCGM's and of the North Atlantic recirculation, can be analyzed with a simple, analytically tractable two-layer model. As explained in more detail in the introduction to Chapter 4, the inertial gyre appearing in the subtropical region, south of the separated Gulf Stream, can be forced by prescribing a potential vorticity anomaly at the western, northern and eastern boundaries of the oceanic basin. The boundary forcing mimics the effect of the Gulf Stream carrying low potential vorticity values, originating in southern latitudes, northward and eastward. Because the bulk of separated Gulf Stream is confined to the thermocline waters, the boundary forcing decays with depth. Nevertheless weak diffusion of momentum acting at the interface between the thermocline and the abyssal waters is able to force the flow down to the ocean bottom.

The onset of motion in the abyssal layer is a strong, highly inertial, barotropic core, about $300 \mathrm{~km}$ wide, with vertically coherent westward velocities of the order of $10 \mathrm{~cm} / \mathrm{sec}$.

The meridional scale of the recirculation is directly proportional to the potential vorticity anomaly and the depth independent part of the transport is proportional to the cube of the abyssal inertial gyre width (see (5.4.8)), in strong analogy with the results found using a homogeneous model. The barotropic core is surrounded by a baroclinic fringe, with strong surface intensified, eastward flows in a region of the order of a deformation radius pressed against the northern boundary of the subtropical gyre. The southern portion of the baroclinic fringe is proportional to $\left(2 \mathrm{H}_{2}-\mathrm{H}_{1}\right) / 2\left(\mathrm{H}_{1}+\mathrm{H}_{2}\right)$ 
and therefore of the order of the recirculation scale, but the flow (westward) is weak. In the westward flowing portion of the baroclinic fringe, the neglected effects of dissipation and of the directly wind driven Sverdrup interior (which is typically easward in this region) may become important.

In a series of numerical experiments I have analyzed the effects of small but finite lateral diffusion of potential vorticity on these boundary driven inertial flows. The global structure of the solution is in excellent agreement with the theoretical prediction, although the actual amplitudes are slightly different. Clearly the agreement is better in the barotropic core, where the velocities are larger, because there the local Reynolds number is higher. The overall impression is that dissipation increases the flow in the barotropic core and decreases it in the baroclinic fringe, with the net result of making the flow even more vertically coherent. This result may depend strongly on the particular type of parameterization for small scale processes, although result from ERCGM's tend to support the choice made here.

Although the vertical resolution of the model is extremely coarse, the results for the barotropic core are independent of the stratification and agree with the barotropic model. Equation (5.4.8) shows that the depth independent transport depends only on the width of the abyssal gyre. In turn, the width of the abyssal recirculation depends, to first order, only on the ratio of the forced layer depth to the total depth of the ocean. It is easy to show that the same result holds for a continuously stratified ocean.

The robustness of the results for the barotropic core depends crucially on the smallness of the baroclinic deformation radius compared to the scale of the recirculation, which is proportional to the prescribed strength of the boundary forcing. In this respect the present theory is incomplete since it's unable to relate the boundary forcing (or the recirculation scale) to external forcing and dissipation, such as the wind, diabatic effects, and boundary current dynamics. 
Appendix A5. Approximate solutions for the gyres widths $\ell_{1}$ and $\ell_{2}$.

In this Appendix approximate solutions of (5.4.5) will be given for the limits: $\alpha_{1} \gg 1$, $r$ arbitrary; $\alpha_{1}$ arbitrary, $r \gg 1$.

Let's first analyze what are the conditions for $\ell_{1}-\ell_{2}=O(1)$, in the limit of $\alpha_{1} \gg 1$. Call $\ell_{1}-\ell_{2}=\delta$ and suppose that $\delta \leq O\left(\alpha_{1}^{-1}\right)$.

From (5.4.4) and (5.4.5)

$$
\begin{aligned}
& \alpha_{1}^{2} \phi \approx\left[\left(1-\ell_{1}\right)\left(1-\cosh \alpha_{1} \delta\right)-\alpha_{1}^{-1} \sinh \alpha_{1} \delta\right]=O(1) \\
& \alpha_{1}^{2} \chi \approx \cosh \alpha_{1} \delta-1-\alpha_{1}\left(\ell_{1}-1\right) \sinh \alpha_{1} \delta=O\left(\alpha_{1}\right) .
\end{aligned}
$$

From (5.4.5b) we have

$$
\ell_{1} \simeq \ell_{2} \approx 3(2+2 r)^{-1}+O\left(\alpha_{1}^{-1}\right)
$$

From (5.4.5a)

$$
\alpha \chi \sinh \alpha \ell_{2} \approx 1-\left(1-\alpha^{2} \phi\right) \cosh \alpha \ell_{2}
$$

and as long as $\ell_{2}$ is $O(1)$, this can be approximated with

$$
\alpha_{1}^{2} \chi \approx \alpha \alpha_{1}^{2} \phi-\alpha_{1}^{2} / \alpha+O\left(e^{-\alpha \ell_{2}}\right)
$$

Substituting from (A5.1) and (A5.2) we get

$$
\left[1-(1+r)^{-1} 3 / 2\right]\left(1-\cosh \alpha_{1} \delta-\alpha^{-1} \alpha_{1} \sinh \alpha_{1} \delta\right)=\alpha_{1}^{2} \alpha^{-2}+O\left(\alpha_{1}^{-1}\right)
$$

By definition $\alpha^{-2} \alpha_{1}^{2}=r /(1+r)$ and after some manipulation one obtains

$$
\sqrt{r /(1+r)} \sinh \alpha_{1} \delta+\cosh \alpha_{1} \delta-1=-2 r(2 r-1)^{-1} .
$$

Eq. (A5.3) doesn't have a solution as long as $r \geq 1 / 2$, therefore when this condition is met the hypothesis made on the relative sizes of the gyres widths is invalid and $\ell_{1}-\ell_{2}=O(1)$. In this case the order of magnitude in (A5.1) is wrong and

$$
\begin{aligned}
& \ell_{1}=1+\alpha_{1}^{-1} \\
& \ell_{2}=3 /(2+2 r)+O\left(\alpha_{1}^{-1}\right)
\end{aligned}
$$


If the lower layer is much thinner than the upper layer, $r \ll 1$, the previous solution would give $\ell_{2}>\ell_{1}$ which is physically unacceptable. In this case the order of magnitude in (A5.1) is correct, (A5.2) is valid and $\delta=\ell_{1}-\ell_{2}$ is the solution of (A5.3). To first order (A5.3) is given by

$$
\sqrt{r} \alpha_{1} \delta+\left(\alpha_{1} \delta\right)^{2}-2 r
$$

and

$$
\delta=\alpha_{1}^{-1} \sqrt{r}(1+\sqrt{5})
$$

Let's now analyze the case $r \gg 1$ with $\alpha_{1}$ arbitrary. In this limit we should recover the results of the one and a half layer model, and we expect $\ell_{1}=O(1), \ell_{2} \ll O(1)$.

We can expand $\chi$ and $\phi$ in powers of $\ell_{2}$ :

$$
\begin{aligned}
& \alpha_{1}^{2} \phi=f_{0}+\ell_{2} f_{1}+\ell_{2}^{2} f_{2} / 2+\ell_{2}^{3} f_{3} / 6 \\
& \alpha_{1}^{2} \chi=g_{0}+\ell_{2} g_{1}+\ell_{2}^{2} g_{2} / 2+\ell_{2}^{3} g_{3} / 6
\end{aligned}
$$

From the definitions of $\phi$ and $\chi$ we get the following relations:

$$
\begin{array}{ll}
f_{1}=g_{0} & g_{1}=\left(f_{0}-1\right) \alpha_{1}^{2} \\
f_{2}=g_{1} & g_{3}=\left(f_{0}-1\right) \alpha_{1}^{4} \\
f_{3}=g_{2} &
\end{array}
$$

Substituting the expansion in (5.4.5b) we get:

$$
\ell_{2}^{2}\left(g_{1} / 2+\ell_{2} g_{2} / 3+\ell_{2}^{2} g_{3} / 6\right)=\left(\alpha_{1} \ell_{2}\right)^{2}\left[\ell_{2}(1+r) / 3-1 / 2\right]+f_{0}
$$

To first order $f_{0}$ must be zero and

$$
1-\alpha_{1}^{-1} \sinh \alpha_{1} \ell_{1}+\left(\ell_{1}-1\right) \cosh \alpha_{1} \ell_{1}=0
$$

which determines $\ell_{1}$ independently of $\ell_{2}$. This is the same relationship found in the one and a half layer model, Eq. (5.3.2). 
In view of this result, the next order of the expansion (A5.4) gives:

$$
g_{2}-\alpha_{1}^{2}(1+r)=\ell_{2} \alpha_{1}^{4} / 2
$$

which determines $\ell_{2}$. Notice that in order for solutions to be possible $\left(\ell_{2}>0\right)$ it must be $g_{2}>\alpha_{1}^{2}(1+r)$ i.e.

$$
r<r_{c}=\cosh \alpha_{1} \ell_{1}-1-\alpha_{1}\left(\ell_{1}-1\right) \sinh \alpha_{1} \ell_{1}
$$

Substituting (A5.5) it can easily be shown that (A5.6) is the same relationship found in Section 3, Eq. (5.3.3). Thus the results from the one and a half layer model are recovered as $r$ is increased past the critical value $r_{c}$ given by (A5.6). 


\section{References}

Böning, C.W., 1982: On the influence of frictional parameterization on wind-driven ocean circulation models. Dyn. Atmos. Oceans, 10, 63-92.

Bower, A.S.,H.T. Rossby and J.L. Lillibridge, 1985: The Gulf Stream - barrier or blender? J. Phys. Oceanogr., 15,24-32.

Fofonoff, N. P., 1954: Steady flow in a frictionless homogeneous ocean. J. Mar. Res., 13, 254-262.

Holland, W.R., T. Keffer and P.B. Rhines, 1984: Dynamics of the oceanic general circulation, the potential vorticity field. Nature, 308, 698-705.

Ierley, G.R. and W.R. Young, 1987: Nonlinear corner flows on a $\beta$-plane. A model of recirculation. Submitted to J. Phys. Oceanogr.

Marshall, J. C. and G. Nurser, 1986: Steady free circulation in a quasi-geostrophic ocean. J. Phys. Oceanogr., 16, 1799-1813.

Musgrave, D.L., 1985: A numerical study of the roles of subgyre-scale mixing and the western boundary current on homogenization of a passive scalar. J. Geophys. Res., 90, 7073-7043.

Niiler P.P., 1966: On the theory of wind-driven circulation. Deep Sea Res., 13, 597-606.

Rhines, P. B. and W. R. Young, 1982: Homogenization of potential vorticity in planetary gyres. J. Fluid Mech, 122, 347-367.

Richardson, P. L., 1985: Average velocity and transport of the Gulf Stream near $55^{\circ} \mathrm{W}$. J. Mar. Res, 43, 83-111.

Roberts, G.O., 1977: Fast viscous convection. Geophys. Astrophys. Fluid Dynamics, 8, 197-233.

Robinson A.R., D.E. Harrison, Y. Mintz and A.J. Semtner, 1977: Eddies and the general circulation of an idealized oceanic gyre: a wind and thermally driven primitive equation numerical experiment. J. Phys. Oceanogr., 7, 182-207.

Schmitz, W. J., 1980: Weakly depth-dependent segments of the North Atlantic circulation. J. Mar. Res., 38, 111-133.

Schmitz, W. J. and W. R. Holland, 1986: Observed and modeled mesoscale variability near the Gulf Stream and Kuroshio extension. J. Geophys. Res., 91, 9624-9638.

Veronis, G., 1966: Wind-driven ocean circulation. Part II: Numerical solutions of the nonlinear problem. Deep Sea Res., 13, 31-55. 


\section{Summary and Conclusions}




\section{Summary and conclusions}

In this thesis I have analyzed some instances in which the Sverdrup relation may fail. In all the cases presented the cause for non Sverdrupian flow can be ascribed to the eddy field, which drives the mean circulation in regions where no external momentum sources are imposed. Other mechanisms that can disrupt Sverdrup relation, such as thermohaline forcing, have not been considered.

In Chapters 2 and 3 the main role of the eddies is to transfer momentum vertically, from the surface waters, which are directly forced by the wind, to the abyssal, unforced waters. Because the mean effect of the eddies is supposed to be weak, substantial abyssal motion occurs only in regions where the geostrophic contours for the abyssal flow are "closed" (see Rhines and Young, 1982). Inside the closed geostrophic contours region the abyssal flow is of the same order as the surface flow and, because of bottom relief, vertical velocities are induced which disrupt the Sverdup relation.

If bottom topography has a longitudnal structure, then internal jets arise with transport of the same order as the maximum interior transport. Two different mechanisms can produce internal jets. If the bottom relief has a constant slope, then the jets are observed at the boundary of the closed geostrophic contours region, and this region is usually well inside the interior of the basin. The reason why the circulation inside the closed geostrophic contours cannot be closed without appending boundary layers is very similar to the mechanism which produces western boundary layers in a closed basin (see Section 2.2). In the presence of $x$-dependent topography, closed geostrophic contours (the circle of radius $r_{1}$ in Fig. 2.3) occur in a region where the total input of vorticity, injected at the surface by the wind and transmitted downward by the interface stress, is non zero. Therefore the abyssal circulation, which has to be contained within the closed geostrophic contours in the inviscid limit, cannot be closed without appending boundary layers. Although the internal boundary layers can occur both in the abyssal and in the surface waters, this is a phenomenon which occurs only in 
baroclinic models. When finite diffusive effects are taken into account the internal jets disappear (see Section 3.3 and 3.4). The same diffusive interfacial stresses responsible for the motion in the abyssal region, render the flow more vertically coherent than it would be in the inviscid limit. Thus the effects that rely on the baroclinicity of the flow are discouraged. On the other hand reduction of diffusion leads to the onset of a strong inertial recirculating gyre (see Fig. 3.8a,b) which acts as a localized source of momentum for the abyssal flow. Due to the presence of such a source the region of abyssal motion is larger than that predicted by the inviscid theory, and the occurrence of internal boundary layers is avoided.

Internal jets can be produced also by a different mechanism if the topographic slope changes sign. Because this phenomenon does not rely on the baroclinicity of the flow, its nature will be explained in the context of a vertically homogenous model. In the presence of topography and planetary vorticity, the large scale geostrophic contours for a homogenous flow are simlpy given by $\beta y+f_{0} h_{b} / H$ where $h_{b}$ is the bottom relief. The free, unforced flow will be along the geostrophic contours. If the topographic slope changes sign then there will be some geostrophic contours which are close by in the interior, but that trace back to regions on the basin boundaries that are far apart (see for example Fig. 3.1b). In general the wind forcing imparted in regions that are far apart will be of different strength. The free component of the flow (the one along the geostrophic contours) induced on the basin boundary by the wind forcing, will be thus very different for geostrophic contours that are far apart on the boundary. Since such free flow component is conserved along the geostrophic contours, in the region where the geostrophic contours get closer, the flow will experience a discontinuity. Clearly in the presence of diffusion such a discontinuity is smoothed, but if diffusion is moderate an internal boundary layer will still be visible. Indeed internal jets can be observed in numerical models which include a moderate amount of diffusion (see Section 3.2) and 
the transport observed in the numerical experiments is in good agreement with the inviscid prediction (Eq. 2.5.3).

In the second part of the thesis, Chapters 4 and 5 , a simple model of the recirculation is developed. In the recirculation region Sverdrup relation fails because inertia becomes important and the direct wind forcing is subdominant. Indeed, due to the inertial character of the recirculation, a gyre can be produced neglecting the local wind forcing. The source of momentum is provided at the boundaries of the gyre and is meant to represent the effect of the boundary current (e.g. the Gulf Stream) carrying low values (in the subtropical gyre) of potential vorticity generated at lower latitudes. The eddy field, which is taken to be weak on average, provides a mean for transfering momentum laterally and vertically, from the localized source to the interior of the gyre. In Chapter 4 a vertically homogeneous model is considered and only lateral momentum transfer is present (this process is absent in the models of Chapter 2). The choice of a vertically homogeneous model of the recirculation is justified by oceanic observations and results from ERGCMs. The homogenous model predicts that the meridional width of the gyre is proportional to the potential vorticity anomaly prescribed at the northern boundary of the gyre, i.e. the separated Gulf Stream. Since this quantity is not known from oceanic measurements, the relation is not of practical use. Nevertheless the model also predicts that the maximum recirculation transport is proportional to the cube of the meridional extent of the gyre (see Section 4.4) and this result is in good agreement with the available observations. Moreover the structure of the zonal velocity, shown in Fig. 4.13, is in qualitative agreement with the observations of Schmitz (1980). Further comparison of the observations with the model results need to be made in order to verify whether the agreements is also quantitative.

In Chapter 5 I tried to answer the question of why the recirculation has a weakly depth dependent structure. To highlight the mechanism acting in a stratified model, I chose to apply the boundary forcing only to the part of the fluid above the thermocline. 
The eddy field tranfers the momentum laterally to the interior of the upper waters gyre as in the homogeneous model, and also vertically to the unforced, deep waters. If the forcing is strong enough to generate a surface gyre with the meridional extent observed in the North Atlantic, then the recirculation extends to the bottom. When this occurs a "barotropic core" is established which carries a very large fraction of the total tranport. The width and tranport of this barotropic flow are the same as those found in the homogeneous model. The most prominent effect of vertical stratification appears in the eastward flow: the eastward maximum velocity occurs at the gyre northern edge and is surface intensified, while the westward flow is quite depth independent. Although direct current meters measurements are absent in the upper waters of the Gulf Stream axis, the analysis of Richardson (1985), who combined current meters and drifting buoys measurements, confirms that, in the recirculation region, the eastward velocity is surface intensified, while the westward return flow is not.

\section{$\underline{\text { Speculations }}$}

One may wonder what flow would be produced by the combined effect of inertia and topography. Some hints on the effect on the basinwide scale are given by the numerical

results presented in Chapter 3. It has been shown that the onset of inertial, recirculating gyres, even if confined to a subbasin region, inhibits the occurrence of large scale internal jets described in Section 2.2. Having failed to observe the baroclinic internal jets in the presence of a localized inertial region, it is unlikely that the jets would appear in a fully inertial regime.

Topography may have an important role in the recirculation region. The structure of the bathymetry in the North Atlantic recirculation region suggests that the deep, inertial gyre may be confined, in the east-west direction, by the bottom topography. Topography cannot be the only parameter which controls the longitudinal extent of the inertial gyre, because, in wind-driven ERGCM's with flat bottom, the recirculation is confined to the western half of the basin. In the same numerical models, the velocity 